\title{
Signes et communication dans les civilisations de la parole
}

Olivier Buchsenschutz,

Christian Jeunesse,

Claude Mordant,

Denis Vialou (dir.) 


\title{
Signes et communication dans les civilisations de la parole
}

\author{
Olivier Buchsenschutz, Christian Jeunesse, Claude Mordant et Denis \\ Vialou (dir.)
}

DOI : $10.4000 /$ books.cths. 1665

Éditeur : Éditions du Comité des travaux historiques et scientifiques Année d'édition : 2016

Date de mise en ligne : 13 novembre 2018

Collection : Actes des congrès nationaux des sociétés historiques et scientifiques

EAN électronique : 9782735508709

\section{Qbooks}

http://books.openedition.org

\section{Édition imprimée}

Nombre de pages : 188

\section{Référence électronique}

BUCHSENSCHUTZ, Olivier (dir.) ; et al. Signes et communication dans les civilisations de la parole.

Nouvelle édition [en ligne]. Paris : Éditions du Comité des travaux historiques et scientifiques, 2016 (généré le 21 septembre 2021). Disponible sur Internet : <http://books.openedition.org/cths/1665>. ISBN : 9782735508709 . DOI : https://doi.org/10.4000/books.cths.1665.

(c) Éditions du Comité des travaux historiques et scientifiques, 2016 Conditions d'utilisation :

http://www.openedition.org/6540 
COMITÉ DES TRAVAUX HISTORIQUES ET SCIENTIFIQUES

\section{Signes et communication dans les civilisations de la parole}

Sous la direction de

Olivier BuCHSENSCHUTZ, Christian JEUNESSE, Claude MORDANT et Denis VIALOU 
Ministère de l'Éducation nationale,

de l'Enseignement supérieur et de la Recherche

Congrès national des sociétés historiques et scientifiques

139e, Nîmes, 2014

Collection Actes des congrès des sociétés historiques et scientifiques,

Version électronique

ISSN 1773-0899 
Introduction,

Olivier BUCHSENSCHUTZ, Christian JEUNESSE,

Claude MORDANT et Denis VIALOU

p. 5

Communicating through rock art:

an ethnoarchaeological perspective

Inès DOMINGO, Sally MAY, Claire SMITH

p. 9

Langage de signes et communication graphique

à la fin du Magdalénien. L'art de Rochereil (Grand-Brassac),

de l'abri Mège et de la Mairie (Teyjat, Dordogne)

Patrick PAILLET, Elena MAN-ESTIER

Les constructions symboliques : l'art préhistorique comme support de communication

Romain PIGEAUD, Florian BERROUET, Estelle BOUGARD

Les mégalithes et leur insertion sémantique dans le paysage.

Langage d'ancêtres

Primitiva BUENO RAMÍREZ, Rodrigo de BALBÍN BEHRMANN

et Rosa BARROSO BERMEJO

Pierres dressées et mâts-totem : le pilier comme vecteur de communication publique dans les sociétés pré-littéraires Christian JEUNESSE

Les signes "à valeur ajoutée » dans l'expression graphique schématique du Néolithique

Philippe HAMEAU 
Faire "parler» les pierres. Le modèle naturaliste en archéologie préhistorique. Le cas de la Vallée des Merveilles, 1868-1913

Maddalena CATALDI

Communication visuelle autour du Camp du Château à Salins-les-Bains (Jura, France) : mise en évidence d'un réseau de relations visuelles à l'âge du Bronze Estelle GAUTHIER et Jean-François PININGRE avec la collaboration de Hervé GRUT

Expression graphique et espace de communication à l'âge du Bronze final. Le bassin rhénan et les Alpes occidentales (du XIII au IX siècle av. J.-C.) Vincent GEORGES

Clamor, couvre-chefs et batterie de cuisine : communication et cryptage des données chez les Celtes de l'âge du Fer Olivier BUCHSENSCHUTZ

La dalle gravée de Teyssieu (Lot), support

d'une communication sans écriture

Gilbert-Robert DELAHAYE 


\title{
Introduction
}

\author{
Olivier BUCHSENSCHUTZ, Christian JEUNESSE, \\ Claude MORDANT et Denis VIALOU \\ Membres de la Section de Pré-Protohistoire du CTHS,
}

\begin{abstract}
Extrait de : Olivier BUCHSENSCHUTZ, Christian JEUNESSE, Claude MORDANT et Denis VIALOU (dir.), Signes et communication dans les civilisations de la parole, Paris, Édition électronique du CTHS (Actes des congrès des sociétés historiques et scientifiques), 2016.

Cet article a été validé par le comité de lecture des Éditions du CTHS dans le cadre de la publication des actes du $139^{\mathrm{e}}$ Congrès national des sociétés historiques et scientifiques tenu à Nîmes en 2014.
\end{abstract}

L'homme du troisième millénaire doit faire un effort considérable pour analyser des sociétés où les moyens de communication n'ont rien à voir avec ceux dont il dispose à ce jour. Les articles regroupés ici traitent de ces communications qui ont prolongé la parole et la mémoire dans la majorité de la population mondiale jusqu'à une date récente. Les grottes ornées, les mégalithes, les mises en scène funéraires, les décors soignés ou les simples grafitti sur des objets de la vie courante, sont autant de messages difficiles à décrypter aujourd'hui. Les quelques exemples retenus illustrent les choix de chaque culture, avec leurs représentations symboliques, réalistes ou idéalisées dont la forme reste très attachée à la culture orale.

L'art pariétal et les mégalithes constituent des supports de réflexion privilégiés pour les manifestations les plus anciennes connues de ces programmes de communication des populations paléolithiques et néolithiques.

Inés Domingo, Sally May et Claire Smith proposent une lecture ethno-archéologique de l'art pariétal actuel d'Arnhem Land en Australie qui permet d'appréhender la fonction exacte de cet art, de comprendre comment l'information culturelle a été incorporée dans son imagerie et surtout comment elle a pu être interprétée par les non-initiés ou par des personnes étrangères à la communauté qui l'a produite. Les résultats de cette étude montrent la complexité des analyses et de l'interprétation de ce type de communication non-verbale et dans quelle mesure les archéologues peuvent décoder les informations contenues dans l'art ancien.

Patrick Paillet et Elena Man-Estier, à partir de quelques exemples tirés de l'iconographie pariétale et mobilière des sites de Rochereil et de Teyjat (La Mairie et l'abri Mège) présentent l'originalité et la puissance du langage des signes et de la communication graphique à la fin du Magdalénien, il y a environ 14000 ans cal BP. Les sociétés humaines, jusqu'alors inféodées aux steppes périglaciaires, s'adaptent peu à peu aux changements environnementaux, repensent également le nouveau monde qui les entoure et inventent de nouveaux symboles.

Romain Pigeaud, Florian Berrouet et Estelle Bougard s'intéressent aux constructions symboliques mises en scène dans l'art préhistorique et ils explicitent la définition proposée par D. Vialou pour qui, les constructions symboliques correspondent à des dispositifs dans lesquels « [...] les liens existant entre les thèmes et entre ceux-ci et leurs supports [...] sont généralement implicites et porteurs de significations ». Ainsi, sur les parois, les associations d'animaux et de signes abstraits ainsi que les traces d'utilisations rituelles dépassent la simple juxtaposition de mythogrammes pour transmettre un message; la roche et le site rocheux ne sont plus de simples supports, ils interagissent avec ce message, pour le nuancer, le modifier, voire le médiatiser pour d’hypothétiques activités rituelles. 
Primitiva Bueno Ramírez, Rodrigo de Balbín Behrmann et Rosa Barroso Bermejo traitent des mégalithes et de leur insertion sémantique dans le paysage à partir d'exemples tirés de la péninsule ibérique. Diverses situations apparaissent : cercles ou alignements qui se transforment en dolmens, menhirs solitaires ou stèles-menhirs, première pierre $\mathrm{d}$ 'un dolmen. Ces exemples parlent de la complexité et de la variabilité de situations dans lesquelles les «vieilles grandes pierres" ont été des marqueurs pendant tout le développement du mégalithisme. Dans la péninsule Ibérique, les marques gravées et peintes, les mégalithes, participent au marquage de territoires occupés dès le Paléolithique supérieur.

Christian Jeunesse propose des comparaisons ethnographiques en relation avec la signification du mégalithisme européen. Il illustre ainsi la diversité des circonstances dans lesquelles sont érigées des pierres dressées ou des piliers de bois et la variété des messages dont ils constituent les supports. Parmi ces populations privées d'écriture, les piliers constituent, à côté de la communication orale, un support privilégié de transmission de la mémoire collective en relation avec les statuts (des vivants ou des morts), les généalogies, les épisodes mythologiques, les événements - anodins ou marquants... Il s'agit donc d'un paysage mémoriel beaucoup plus coloré et divers que ne le laisse supposer l'austérité de nos mégalithismes « fossiles » européens.

Philippe Hameau dresse un corpus iconographique du Néolithique, peint ou gravé, qui se restreint à quelques catégories de figures: les êtres vivants (hommes, animaux et « idoles ») et les signes (motifs soléiformes et lignes brisées). Les uns et les autres peuvent être figurés dans des versions très réalistes, au sens d'immédiatement reconnaissables, ou très simplifiées au point de nous paraître absconses. La syntaxe de cette iconographie est perceptible au travers de règles précises d'apposition des figures; ces processus associatifs conduisent à penser que les motifs soléiformes et les lignes brisées indiquent le statut des êtres vivants : l'étape de leur passage et leur transformation sur et par le site sur lequel ils sont figurés. Il semble que cette même valeur ajoutée véhiculée par les mêmes signes soit reprise pour l'iconographie linéaire médiévale.

Maddalena Cataldi porte son attention sur les premières études consacrées aux très célèbres gravures du Mont Bégo en relation avec le développement du modèle naturaliste en archéologie préhistorique. C'est au milieu du XIX ${ }^{\mathrm{e}}$ siècle que les gravures du site de la vallée des Merveilles, connues depuis le XVII ${ }^{\mathrm{e}}$ siècle, sont interprétées comme des vestiges préhistoriques par la communauté scientifique émergente des préhistoriens. Cette contribution explore la relation entre la construction du savoir sur l'Homme préhistorique et la construction des outils conceptuels susceptibles de permettre la connaissance de ses cultures. Parmi ces outils, les types, ensembles d'objets, rassemblés en raison de leur proximité morphologique sont rapprochés des taxons utilisés par les sciences naturelles (espèces, genres, etc.) dans le discours de l'archéologie préhistorique en devenir.

Estelle Gauthier, Jean-François Piningre, en collaboration avec Hervé Grut, abordent un concept original, celui de la communication visuelle autour du Camp du Château à Salins-les-Bains (Jura, France) à l'âge du Bronze. Dans le secteur de ce typique habitat fortifié, des prospections systématiques ont permis la mise au jour de 40 dépôts de l'âge du Bronze. La bonne précision de leur localisation permet de mesurer les liens de perceptibilité entre les lieux de dépôt et des points marquants du paysage naturel et social de l'âge du Bronze, en particulier des sites de hauteur. Les dépôts semblent marquer les frontières de l'espace visible depuis le Camp du Château. La mise en place d'une forme de communication visuelle entre les sites a pu permettre de soutenir la mémoire communautaire et le choix d'implantation des dépôts aurait pu avoir pour objectif de baliser l'espace social et culturel associé au camp de hauteur fortifié.

Vincent Georges porte son attention sur les décors figurés sur les bracelets au Bronze final (XIII ${ }^{\mathrm{e}}$ au IX ${ }^{\mathrm{e}}$ siècle av. J.-C.) sur un large espace, se déployant du bassin rhénan aux 
Alpes occidentales. Ces décors et leurs importantes transformations seront lus comme une influence des élites car, sur métal ou textile, ces graphismes complexes seront perçus et interprétés selon une hypothèse de la transmission du pouvoir par voie utérine. Le rôle de certaines femmes aboutirait au perfectionnement de leur apparat sur le long terme et les «sémioses graphiques" présentées, tiraillées entre innovations et traditions se déploieraient au gré des échanges matrimoniaux de haut rang dont elles enregistreraient tout à la fois, la complexité et la réalité.

Olivier Buchsenschutz aborde la communication chez les Gaulois aux travers de données archéologiques et de la rhétorique césarienne. César insiste sur la communication par le son, et sur les limites de l'utilisation des documents écrits ; on en a un peu rapidement déduit que les Gaulois n'avaient pas atteint le stade de l'écriture alors que les nouvelles données archéologiques révèlent son utilisation plusieurs siècles avant la conquête romaine. Au premier âge du Fer, l'analyse des mises en scène funéraires révèle un jeu subtil entre la présence matérielle des composantes du banquet ou leur représentation sur des vases. Â partir du IV siècle av. J.-C., le récit disparaît des images ; le banquet est représenté directement par la nourriture et les instruments de cuisine. Le décor des armes et des parures joue avec l'abstraction, la double lecture, et une symbolique réservée aux initiés.

Pour clore ce volume, Gilbert-Robert Delahaye retrace les avatars d'une dalle approximativement circulaire retrouvée à proximité du château de Teyssieu (Lot), qui montre, gravé sur une de ses faces un tablier de jeu de trictrac d'âge médiéval dotées de cupules. L'objet aurait ensuite été réemployé pour indiquer, à une époque récente, par le dépôt d'un caillou dans telle ou telle cupule, le lieu où le pâtre communautaire menait les animaux qu'il prenait en charge. La dernière fonction de cette pierre s'est transmise localement par voie orale et bien que constituant une sorte de document cartographique, celui-ci n'est pas réaliste du fait de la première utilisation de la gravure. On ignore le lieu où elle était initialement exposée et l'époque où elle fut utilisée, sans doute avant la généralisation de l'alphabétisation; elle constitue, quoi qu'il en soit, une forme de communication sans écriture des plus originales. 


\title{
Communicating through rock art: an ethnoarchaeological perspective
}

\author{
Domingo INÈS, \\ ICREA Research Professor at Universitat de Barcelona \\ Department of Prehistory, Ancient History and Archaeology/SERP
}

May SALLY The Australian National University School of Archaeology and Anthropology

Smith CLAIRE,

Flinders University Department of Archaeology

\author{
Extrait de : Olivier BUCHSENSCHUTZ, Christian JEUNESSE, Claude MORDANT et Denis VIALOU (dir.), \\ Signes et communication dans les civilisations de la parole, Paris, \\ Édition électronique du CTHS (Actes des congrès des sociétés historiques et scientifiques), 2016. \\ Cet article a été validé par le comité de lecture des Éditions du CTHS dans le cadre de la publication \\ des actes du $139^{\mathrm{e}}$ Congrès national des sociétés historiques et scientifiques tenu à Nîmes en 2014
}

\begin{abstract}
In many past and present preliterate societies rock art has been used as a means of communications to convey ideas, believes, ancestral knowledge, traditions, identities, rules, stories, social behaviour or laws. Through an ethno-archaeological perspective based on Arnhem Land rock art, we explore the different functions of the art, how cultural information is embedded in the imagery and more importantly, to what extent the information can be decoded by an uninitiated or an outsider to the culture producing the art. The results of this research provide insight into the complexities of analysing and interpreting this form of non-verbal communication including the extent to which archaeologists can decode cultural information hidden in past forms of art.
\end{abstract}

Keywords : Rock art, ethnoarcheology, Arnhem land, Australia

\section{Résumé}

Dans de nombreuses sociétés anciennes et plus récentes de tradition orale, l'art pariétal a été utilisé comme un moyen de communication pour véhiculer des idées, des croyances, du savoir, des traditions, des identités, des règles, des histoires, des comportements et des lois. Une lecture ethno-archéologique de l'art pariétal d'Arnhem Land nous a permis d'appréhender la fonction exacte de cet art, de comprendre comment l'information culturelle fut incorporée dans son imagerie et surtout comment elle a pu être interprétée par les non-initiés ou par des personnes étrangères à la communauté qui l'a produite. Les résultats de cette étude montrent la complexité des analyses et de l'interprétation de ce type de communication non-verbale et dans quelle mesure les archéologues peuvent décoder les informations contenues dans l'art ancien. (Traduction Rebecca PEAKE) 
This paper explores the role of rock art as communication and evaluates the challenges to decode meaning in situations where we are unfamiliar with the culture that produces de art, either in the past or in the present.

Rock art is a visual medium used by past and present pre-literate societies as a nonverbal form of communication and information exchange (Conkey 1978, 1980 ; Gamble 1982 ; Smith 1992 ; Domingo 2012). During generations it has been used to illustrate stories or to graphically represent different sorts of cultural information to educate, remind, regulate, celebrate and so forth. As summarized in the seminal text by Ucko and Rosenfeld (1967:7) rock art includes both religious and secular imagery, as well as a wide range of signs that assist humans to adapt, coexist and share specific socio-cultural and natural landscapes.

As any form of communication rock art requires a sender (the person who encodes the information, which in this case will be the artist/s), a message (the information or content to be shared), a channel (the art forms) and a receiver/s (who decode/s the message). For the transmission of information to have maximal effectiveness the interacting agents (sender and receiver/s) need to share a common set of signs, symbols or language. But what happens when there is no common cultural background among the interacting agents? It is likely that the messages would become distorted during the communication process, thus preventing effective communication.

The depiction of figurative motifs inspired by real object sources may allow literal readings of the art (i.e. a literal identification of the motif as "a deer, a goat, a kangaroo, a human" or an action "a war scene, a dance, a ceremony, a hunting scene"). However, this in itself is problematic, as MacIntosh (1977) demonstrated in his reanalysis of the motifs at Beswick Creek Cave, in the Northern Territory, Australia, when he found that $90 \%$ of his interpretations undertaken in 1952 without a local teacher, were incorrect.

Moreover, even if a motif is correctly identified this does not necessarily mean that the communication is effective, since it is likely that the image encodes a symbolic message that is unreadable to an outsider who is not initiated into the knowledge system. For example, the image of a deer may represent a prey, but it may also represent a spirit or an ancestor, a desired strength or ability, the identity of an individual or a group, and so forth. The likelihood of successful communication is even more tenuous when depictions include non-figurative objects (geometric and abstract depictions), which are interpreted as a simplification or abstraction of the concept to be shared.

This raises the question: Is it possible for outsiders to understand the symbols of a different culture?

When travelling to a country where we are unfamiliar with the language, the signs and / or the culture, we all experience some sort of miscommunication. Images and signs can become meaningless to us, and thus the communication process is broken. The same motif or sign can embrace multiple meanings that vary according to the context or the culture. When the same animal is depicted in the imagery of several cultural groups we need to analyse the context to understand the specific meaning. Thus, for example, the image of a red rooster in an Australian sign refers to a place to eat roasted chicken: the Red Rooster restaurants. The same animal depicted in a church, like the rooster sculptured in one of the doors of the Gaudi's famous Sagrada Familia church in Barcelona, is linked to a religious story: the rooster crowing upon Saint Peter's third denial of Christ. Finally, the Portuguese rooster known as the Galo de Barcelos is often used as a symbol of Portugal, and thus represents a cultural identity (fig. 1). So in a contemporary setting, representations of the same animal are used in different countries and cultures to refer to very different things: food, a myth or an identity.

This sort of miscommunication also happens when trying to interpret the past through the analysis of archaeological remains. While when travelling to a different country a 
local guide can inform us of the meaning of the images, when travelling to the past we can only rely on the archaeological context, which may inform us about the potential function of the art, but rarely about the symbolic meaning (Domingo and May 2008).

In this paper we use our ethnoarchaeological research in the Arnhem Land and Barunga regions of the Northern Territory (Australia) to argue that focusing our research efforts on deducing the meaning of the art only leads the discipline to a dead end. Moreover it overshadows the potential of rock art to explore other aspects of past societies such as their natural and cultural environment, their material culture, including the identification of ephemeral material culture that is underrepresented in the archaeological record; technological developments, social boundaries and territorial behaviours, and so forth.

\section{Methods}

Today, there are only a few places in the world where rock art is still part of the symbolism of a living culture and where we can still (to varying extents) access the meaning of the art guided by the knowledge of the authors of this art or their descendants. The Arnhem Land and Barunga regions of the Northern Territory (Australia) are two of these few places. This is where we will travel in this paper to illustrate our discussion.

Using several examples from Arnhem Land and the Barunga region we will explore the different functions of rock art, how cultural information is embedded in the imagery and to what extent the information in the art can be decoded by an outsider to the culture that produced the art.

To answer these questions we follow a three step method:

- The first step is to select the sites, record the motifs and scenes and analyse them from an archaeological perspective (i.e. through description and quantification of motifs, techniques, themes, patterns of composition and addition and so forth). A detailed example of this process can be found in Domingo (2008), May and Domingo (2010) and Domingo (2011). At this point our aim is to test the extent to which we can identify the motifs and scenes depicted from a purely archaeological analysis.

- The second step is to analyse the archaeological context, looking for evidence of human activities to deduce the potential uses of the site, and thus suggest hypothesis concerning the function of the art.

- The third step is to conduct ethnoarchaeological research to attempt to record the meaning/s that Indigenous populations assign to the motifs, themes, scenes or sites under study, to test the validity and limits of our interpretations. To do this, we combine oral interviews with Indigenous elders and artists, with a literature review including early ethnographic records.

\section{Introduction to the research area}

The research presented in this paper was conducted in Australia. Prior to European contact Australian Aboriginal and Torres Strait Islander groups showed great cultural diversity, with different languages, cultural practices and material culture, as demonstrated in Horton's (1999) map of Aboriginal Australia. While they were mostly semi-nomadic, they had strong connections to particular parts of the country. At the time of contact there were numerous rock art traditions in Australia, with more geometric art system in the centre and a range of different forms of geometric and figurative art in other regions (Morwood 2002). Our research focuses specifically on the Western Arnhem Land and the Barunga regions of the Northern Territory, where figurative painted rock art predominates (fig. 2), though there are also some engravings or abraded grooves. 
Unlike other Australian territories, where the arrival of Europeans severely impacted on the Indigenous population and their culture, both Arnhem Land and the Barunga region were declared as Aboriginal reserves in the 1930s. These declarations attenuated the impact of European invasion, allowing the continued operation and continuation of one of the longest rock art traditions in the history of mankind.

Over a period of around 50,000 years (Roberts et al. 1990 : 153 ; Roberts et al. 1998) the Arnhem Land and Barunga regions were inhabited by a variety of Indigenous populations with an economy based on hunting and gathering. These populations organized in flexible bands and practiced some sort of mobility in search of seasonal resources (see, for example, Chaloupka 1981). While their technology was relatively modest, they developed one of the most sophisticated socio-cultural lives recorded by anthropologists, with a significant investment of time in cultural practices, including art, religion and laws (Morphy 1992 ; Flood, $1997: 2$; Smith 2002, 2004). In these societies, ceremonies played a significant role in the transmission of knowledge and cultural practices. In ceremonies religion, history and laws were melted through dance, music, stories and various forms of art (body art, rock art and portable art) to ensure the appropriate training of the new generations, the exchange of ideas and raw materials and appropriate rituals regarding the various stages on life (see, for example, Berndt and Berndt 1970 ; Morphy 1992 ; Smith 2004 and Taylor 1996).

The arrival of Europeans in the Northern Territory in the 1880s had a significant impact on Indigenous ways of life. However, interruption of their traditional economies and a general transformation into a more sedentary lifestyle did not cause the disappearance of their beliefs and socio-cultural practices. On the contrary, Indigenous identities were reinforced in comparison to the "others" and continued to ensure the transmission of ancestral knowledge to new generations (Taylor 1996; Domingo and May, 2008; May 2008). This cultural continuity offers a unique context to test to what extent an archaeological approach to rock art is useful in interpreting the rock art of a different culture, when we lack information about their socio-cultural and symbolic traditions.

\section{Functions and interpretations of rock art in Arnhem Land}

In Arnhem Land, rock art has been used in different contexts to convey ideas, beliefs, ancestral knowledge, traditions, identities, stories, social behaviour and laws. As local Elder Thompson Yulidjirri explained to one of us, the art is not an end, but a gateway (May, field diary 2001, May 2006: 43).

The meaning of this art is mostly related to the so-called 'Dreaming', an English term for the creation era in which ancestral beings travelled the earth producing its topographic features (Morphy 1992). Much rock art of these regions is the graphic expression of beliefs, sacred values and rules the creation beings passed on to humans (Smith 2000). Thus, to interpret the art it is absolutely necessary to be familiar with the Indigenous culture and beliefs system.

The imagery of the Arnhem Land and Barunga regions include Ancestral Beings as well as evil spirits, usually full of sacred symbolism. In Arnhem Land, Yingarna (the creation mother) is one name for the ancestor whose journey resulted in the creation and dissemination of Aboriginal people and language groups in northern Australia (fig. 3). She is said to have emerged from the sea to the north and travelled inland, teaching people language and clan. She can adopt different physical forms, either a female figure or a rainbow serpent. Thus, both images would evoke the same story to a knowledgeable audience, whereas to an outsider they would be identified as completely different motifs with no evidence of a link between them, or to the Yingarna narrative. 
Similarly, in some parts of this country Milingi can be portrayed as either good or bad spirits. For Rembarrnga people they are good, but at Barunga they are often thought of as mischievous. For example, they can trick you and can turn the landscape around so you get lost (fig. 4). Visually speaking, they are human like forms. For someone familiar with this culture they are easily identifiable since their rib bones are shown, whereas they are not shown for people. Once again, without ethnographic information all an outsider can tell is that they are different to other human figures. We could easily analyse their spatial distribution and their place in the artistic sequence to explore questions of cultural change and territorial behaviour, but we will be completely unable to obtain meaning per se.

In these territories, there are other secular representations that reflect Indigenous concerns (love magic, commemoration of events, educational purposes) or representing daily life (hunting or fishing scenes). These sorts of images are said to be produced to fill the time or for story telling in public contexts (Chaloupka 1993), thought it is possible that they also have other non-public levels of meaning.

Sorcery figures are usually human beings depicted in strange or inverted postures, sometimes with distorted genitalia (fig. 5). The aim of these representations is to cause illness or death to another person for a range of reasons (Chaloupka 1993). It would be difficult for an outsider to deduce this magical purpose by simply visualizing the painting, though they could record stylistic differences of these images in comparison to other human-like figures.

Rock art in the Northern Territory also has numerous examples of plant and animal resources that have been used for generations as food or medicine. At Injalak Hill in Arnhem Land, representations of fish, either in pieces or complete, are used to teach how to process the meat and which parts are the most valued when distributing the food (fig. 6). But fish are more than food, they are powerful symbol of life. Fish can be tied to maps showing the territories of various clans, or they can be used to tell stories of the creation time, and so forth (Taçon 1994: 124).

Sometimes the motifs depicted in these regions represent objects we are not familiar with (fig. 7). In such a situation only Indigenous people can help us to interpret them.

We are in a similar situation when we move from the interpretation of individual motifs, to the interpretation of compositions and scenes, and sometimes even when we are interested in knowing the function of some sites. An Indigenous teacher is necessary to understand the themes depicted or the function of some sites, as exemplified in our discussion below.

At the site known as Djulirri (Anuru Bay) there is a scene featuring various white human figures, with stick like bodies and no formal differences among them (fig. 8). Analysing the distribution of the motifs and their actions, we could guess of a possible fight (a complex battle). However, Indigenous elders informed us that the interpretation of this scene is far more complex. In fact, it is a ceremony related to a funeral. Thus, ethnographic information is key to interpreting the activity depicted in this scene as a ceremony instead of a war. Similarly, only with ethnographic information could we interpret the circle depicted in front of the face of the two leading individuals in the top right confrontation. In Arnhem Land, 'biting bags' (also known as power bags or spirit bags) filled with feathers or vegetable fibres and stitched closed were worn by men during certain ceremonies (Hamby, 2011 : 227). It would be placed between a man's teeth when he was fighting to give him power and courage. Since most of the rituals relating to this ceremony are restricted, further details were not discussed with Indigenous teachers. Moreover, such details were not necessary for our research.

Another scene at the Djulirri site includes several stick like human figures, with differences in size, action and in the objects they are holding (fig. 9). Individuals on the 
left are holding an object on top of their heads and seem to be performing some sort of dancing. Thanks to our previous knowledge of Indigenous traditions we were able to recognise some of the objects being held by some of the individuals on the right as musical instruments. The second individual from right to left is holding a didgeridoo (a musical wind instrument traditional to this Australian region). The fourth individual is holding clap-sticks (a musical percussion instrument that men strike to accompany the didgeridoo during their ceremonies). So from these two instruments, and analysing the distribution of the motifs and their action we deduced that the group on the right were musicians and that the scene was depicting some sort of ceremony. However, we were not familiar with either the objects held by the group on the left, nor with the ceremony depicted. From our archaeological analysis we could not really tell much about this scene, apart from guessing that there might be social differences between the biggest and the smallest individuals depicted, perhaps due to age or social status.

Through ethnography we were informed that the ceremony depicted was the Ubarr ceremony, although further details about this ceremony were not discussed.

Far from this region, and travelling to the south, in the land near the Aboriginal communities of Barunga and Wugularr there is another interesting site that provides an example of the role of ethnography in interpreting both the meaning of the art, and the function of the site. The Mook Mook site depicts a creation story: the creation of owls, which are being 'vomited' by the rainbow serpent (fig. 10). The motifs at this site are quite naturalistic, and while an outsider familiar with these animals may be able to identify them, in the past they have been erroneously identified (Davidson 1981). However, it is only with ethnographic information that it is possible to identify the narrative represented. Furthermore, ethnography also is necessary to understand the function of this site. An archaeozoologist could easily deduce that the bones deposited in the base of the painted panel are kangaroo bones, and all of them correspond to the same anatomical part. An archaeologist might surmise that this is a ceremonial site from the arrangement of kangaroo femurs and the fact that they are coated in red ochre. However, there is a big step from these observations to the ethnographic knowledge that the site is related to an initiation ceremony for young boys, in which the boy's mother has collected the femurs of the kangaroos hunted by the son as he proves himself to be a good hunter and ready to start a family.

\section{Results and discussion}

In this paper we have used ethnoarchaeology to argue that rock art research should go beyond the search for meaning in rock art, to explore more quantitative and qualitative aspects of this form of archaeological remains.

Through our research with Indigenous elders and artists in the Arnhem Land and Barunga regions of the Northern Territory we have learnt that visual arts are a mean of communication that can be used for a wide range of purposes : to illustrate a story, to mark a place, to provide memory of an event, to depict rules for how to behave and to communicate ownership or identity. The meaning and function of the art change according to the context or the culture. Thus, to obtain meaning from rock art it is necessary to be familiar with the cultural beliefs and traditions in which the art was conceived.

Through different case studies we have demonstrated that while some of the information encoded in rock art can be perceived from an archaeological analysis of motifs or scenes and the archaeological context, a full comprehension of the beings, traditions and activities represented in rock art and the complex social rules and regulations depicted can only be achieved through a full understanding of the cultures who produced the art. In our view, focusing on finding the meaning of prehistoric art masks the potential of rock art to provide information on other quantitative aspects of past societies, such as 
territorial behaviour, technological practices, material culture, natural environment and so forth.

Acknowledment

Information for the motifs and scenes shown in this paper was provided by several Indigenous Elders and Artists from Gunbalanya, The Wellington Range, Barunga and Wugularr (Arnhem Land), who approved the use of this information for publication. Unfortunately a significant number of them have past away in the last few years and out of respect we have not use their names in this paper. We are thankful to all of them for working with us and opening our eyes to their traditions and culture.

This paper has been produced in the context of the Project HAR2011-25440 (Ministry of Science and Innovation, Spain).

\section{Bibliographie}

BERNDT R.M. and BERNDT C.H. (1970). Man, land \& myth in North Australia. The Gunwinggu people, Sydney, Ure Smith.

CONKEY M.W. (1978). Style and information in cultural evolution: towards a predictive model for the Palaeolithic, in C. REDMAN et al (eds.), Social Archaeology: beyond subsistence and dating, London, Academic Press, p. 61-85.

CONKEY M.W. (1980). Context, structure and efficacy in Palaeolithic art and design, in M. FOSTER and S. BRANDES (eds.), Symbol as Sense, New York, Academic Press, p. 225-248.

CHALOUPKA G. (1981). The traditional movement of a band of Aboriginals in Kakadu, in T. Stokes (ed.), Kakadu National Park Education Resources, Canberra, Australian National Parks and Wildlife Service, p. 162-171.

ChaloupKa G. (1993). Journey in Time: The World's Longest Continuing Art Tradition: The 50,000-Year Story of the Australian Aboriginal Rock Art of Arnhem Land, Reed New Holland, Sydney.

DOMINGO I. (2008). From the Form to the Artists: changing identities in Levantine rock art (Spain), in I. DOMINGO, D. FIORE and S. MAY (eds.), Archaeologies of Art. Time, place and identity. Series One World Archaeology, California, Left Coast Press, p. 99-129.

DOMINGO I. (2011). The rock art scenes at Injalak hill: alternative visual records of Indigenous social organization and cultural practices, Australian Archaeology, nº 72, p. 1522.

DOMINGO I. (2012). A theoretical Approach to Style in Levantine rock art, in J. MCDONALD and P. VETH (eds.), A companion to rock art, Boston, Blackwell companions to Anthropology, p. 306-322.

DOMINGO I. and MAY S.K. (2008). La pintura y su simbología en las comunidades de cazarecolectores de la Tierra de Arnhem, in J. SAlAZAR, I. DOMINGO, J. AZKÁRRAGA and H. BONET (eds.), Mundos Tribales: Una Visión Etnoarqueológica, Valencia, Museu de Prehistoria, p. 78-91.

FlOOD J. (1997). Rock art of the Dreaming. Images of Ancient Australia, Sydney, Angus and Robertson.

GAMBLE C. (1982). Interaction and alliance in Palaeolithic society, Man (N.S.), n ${ }^{\circ} 17$, p. $92-$ 107. 
HAMBy L. (2011). The Forgotten Collection: Baskets reveal histories, in M. THOMAS and M. NEALE (eds.), Exploring the Legacy of the 1948 Arnhem Land expedition, Canberra, ANU E Press, The Australian National University, p. 213-238.

HORTON D. (1999). Map of Aboriginal Australia. Available at: http:/ / www.aiatsis.gov.au/asp/map.html. Accessed 14 September, 2014.

MAY S.K. (2006). Karrikadjurren - Creating Community with an Art Centre in Indigenous Australia, Unpublished PhD thesis, Canberra, Centre for Cross-Cultural Research, Australian National University.

MAY S.K. (2008). Learning Art, Learning Culture: Art, Education, and the Formation of New Artistic Identities in Arnhem Land, Australia, in I. DOMINGO, S.K. MAY and D. FIORE (eds.), Archaeologies of Art: time, place and identity, Left Coast Press, Walnut Creek CA, p. 171-194.

MAY S.K. and Domingo I. (2010). Making sense of scenes, Rock Art Research, n 27.1, p. 3542.

Morwood M. (2002), Visions from the Past. The archaeology of Australian Aboriginal Art, Sydney, Allen \& Unwin.

Roberts R. G., JONES R. and SMith M. A. (1990). Thermoluminescence dating of a 50,000year old human occupation site in northern Australia, Nature, $n^{\circ} 345$, p. 153-56.

Roberts, R., YoshidA H., GAlbraith R., LASLETT G., JONES R. and SMITH M. (1998). Single-aliquot and single-grain optical dating confirm thermoluminescence age estimates at Malakunanja II rock shelter in northern Australia, Ancient TL, nº 16, p. 19-24.

SMITH C. (1992). Colonizing with style: reviewing the nexus between rock art, territoriality and the colonisation and occupation of Sahul, Australian Archaeology, no 34, p. 34-42.

Smith C. (2000). The Art of the Dreaming, Cover article commissioned for Scientific American Discovering Archaeology, April, 2000: 31-7.

SMITH C. (2004). Country, Kin and Culture. Survival of an Australian Aboriginal Community, Adelaide, Wakefield Press.

TAÇON P.S.C. (1994). Socialising landscapes: the long term implications of signs, symbols and marks on the land, Archaeology in Oceania, n 29, p. 117-129.

TAYLOR L. (1996). Seeing the Inside: Bark Painting in Western Arnhem Land, Oxford, Clarendon Press.

UCKO P. and Rosenfeld A. (1967). Palaeolithic Cave Art, London, World University Library. 


\section{Illustrations}

Figure 1: The same motif can have different meanings according to the context and culture. A. Rooster. B. Symbol of the Australian restaurant Red Rooster. C. Rooster sculptured in the Sagrada Familia Church (Barcelona). D. Portuguese rooster.

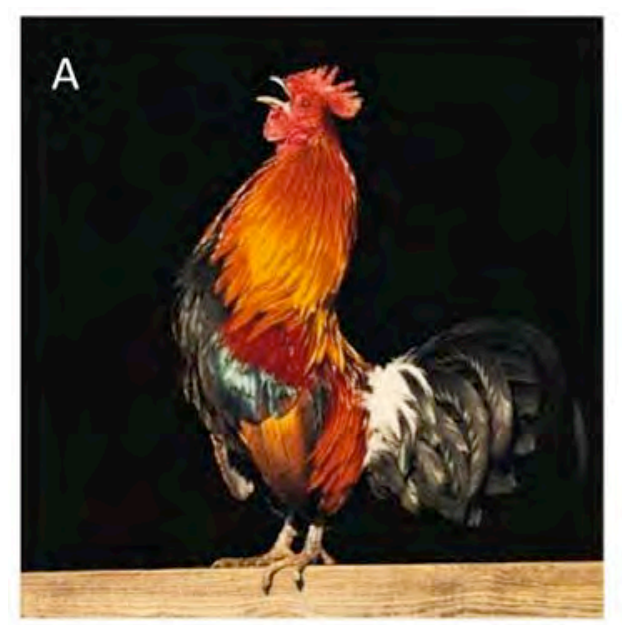

B
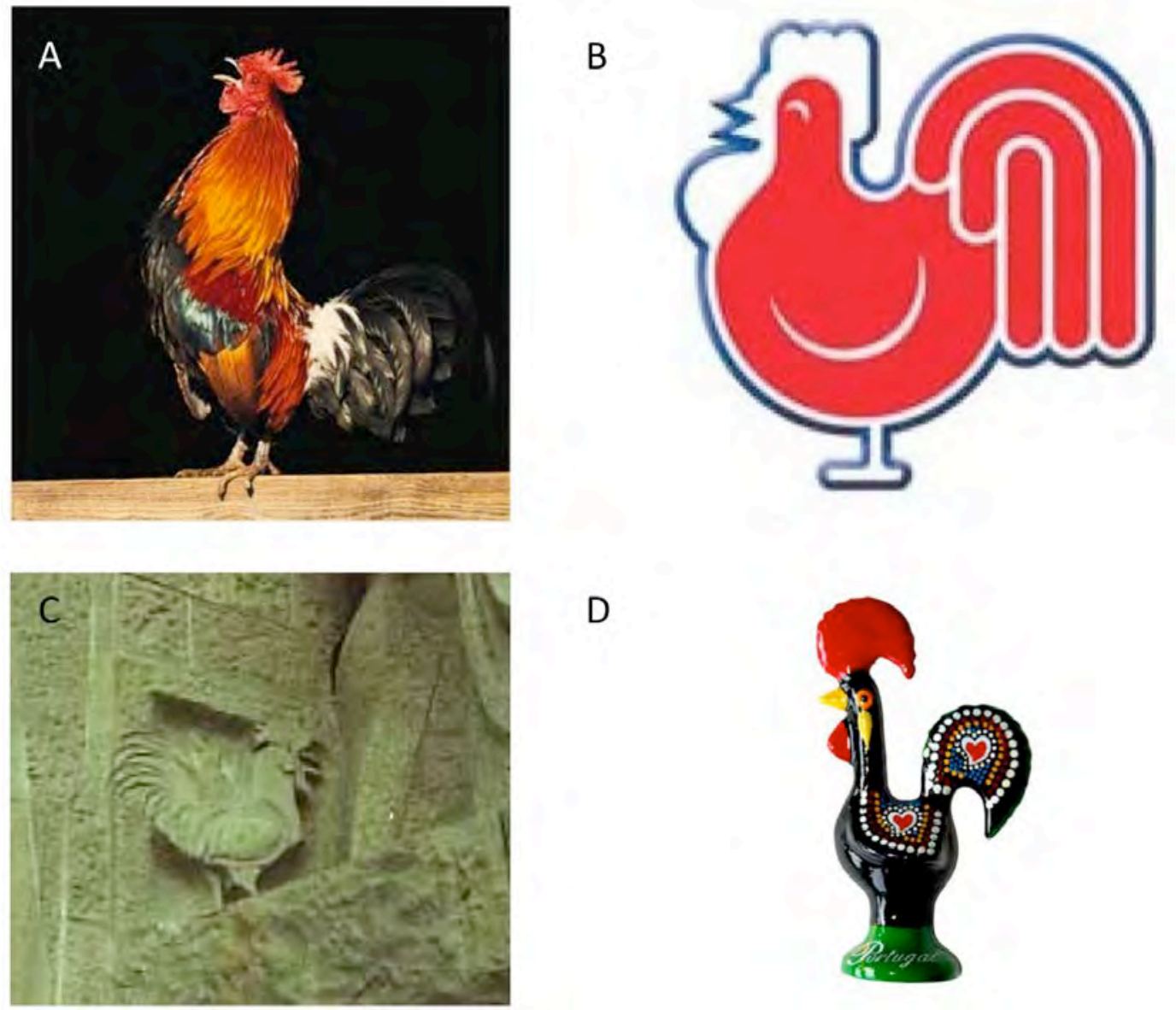

D

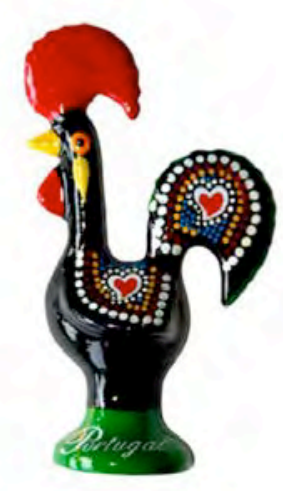


Figure 2: Map showing the location of the research area. (Map from Chaloupkak 1993, modified).

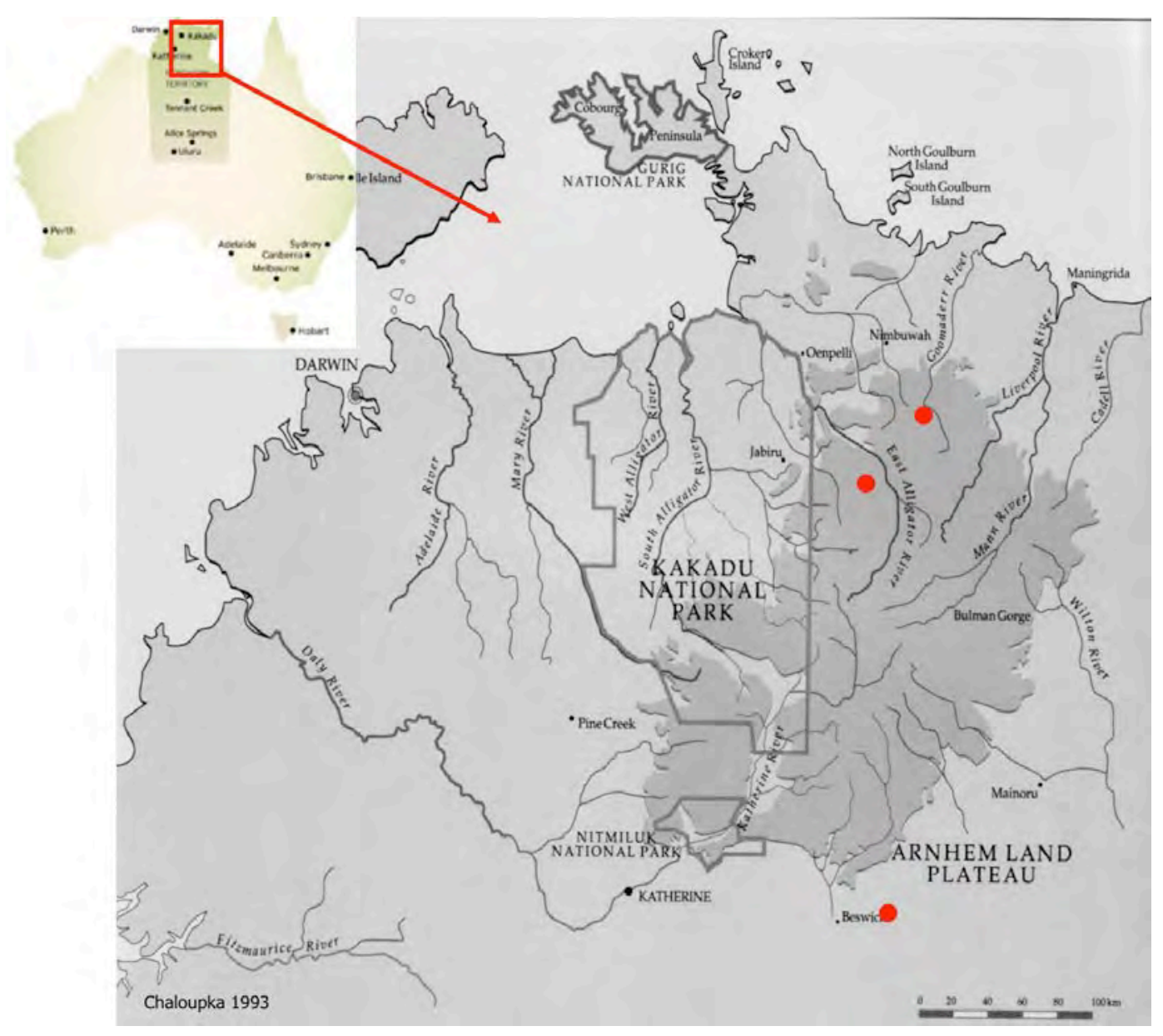


Figure 3. Yirgana (the creation mother) (Injalak Hill, Gunbalanya). Photograph by I. Domingo.

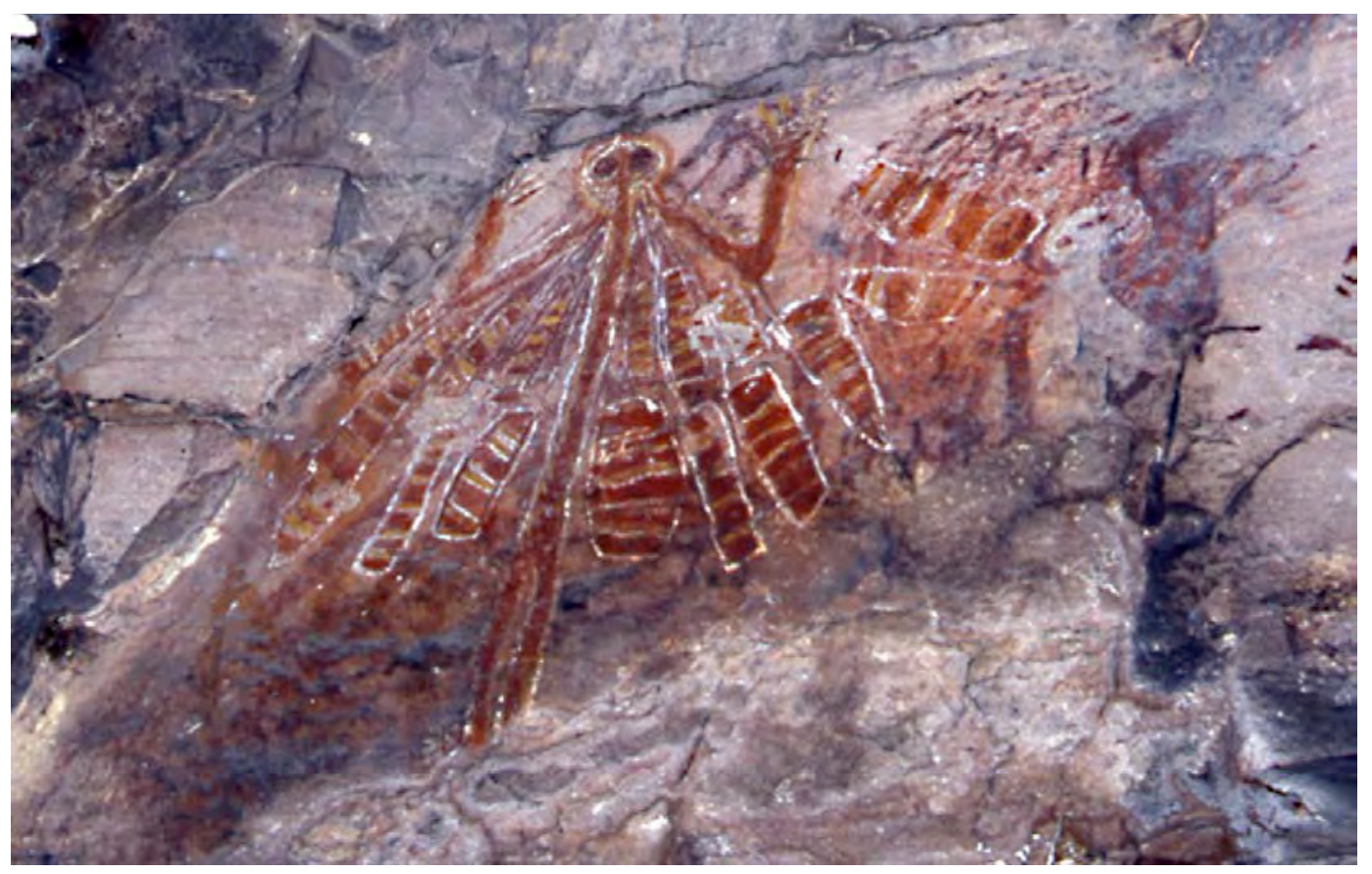


Figure 4 : Milingi (bad spirits) (Drupni site, Barunga region). Photograph by I. Domingo.

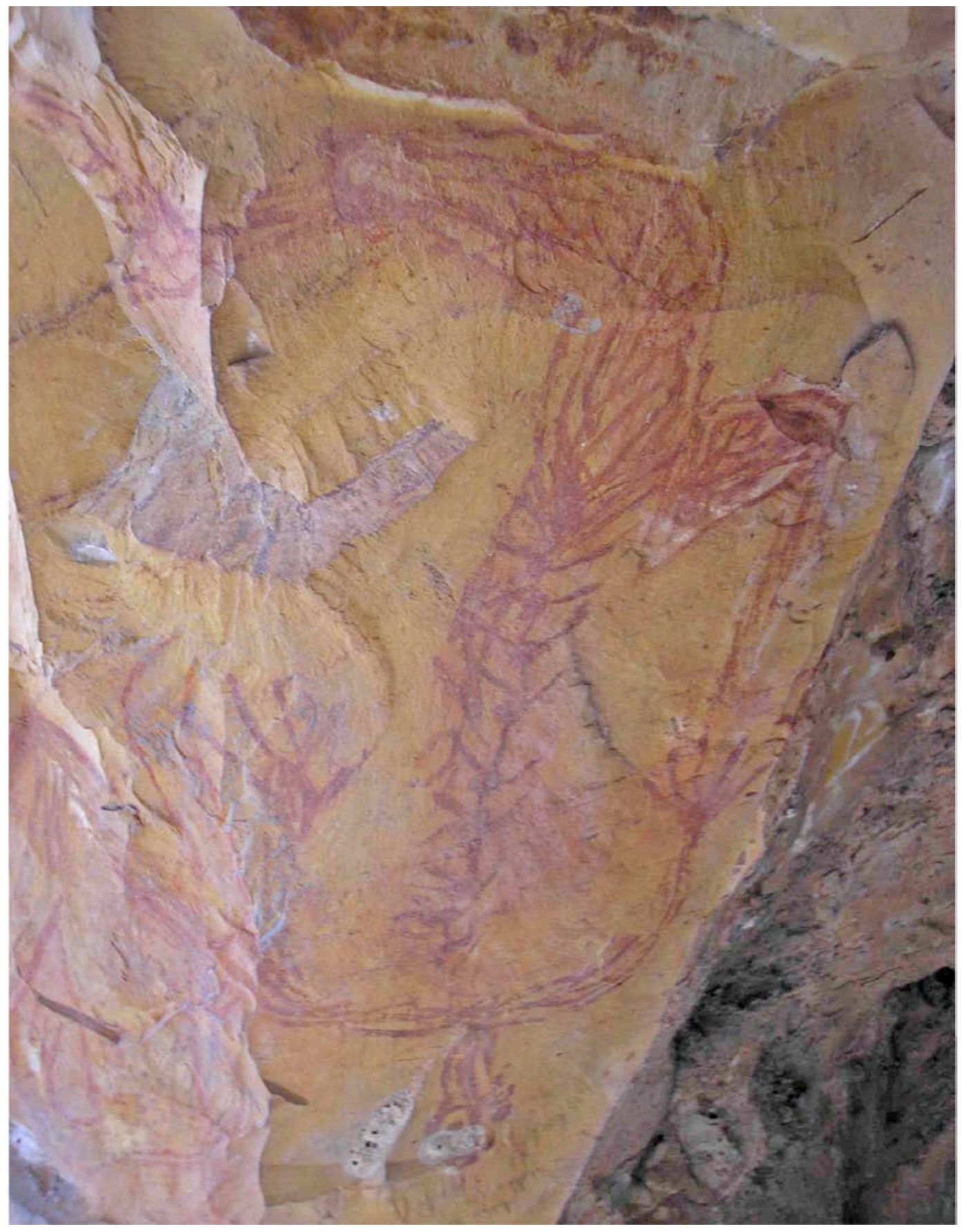


Figure 5 : Sorcery figure (Injalak Hill, Gunbalanya). Photograph by I. Domingo.

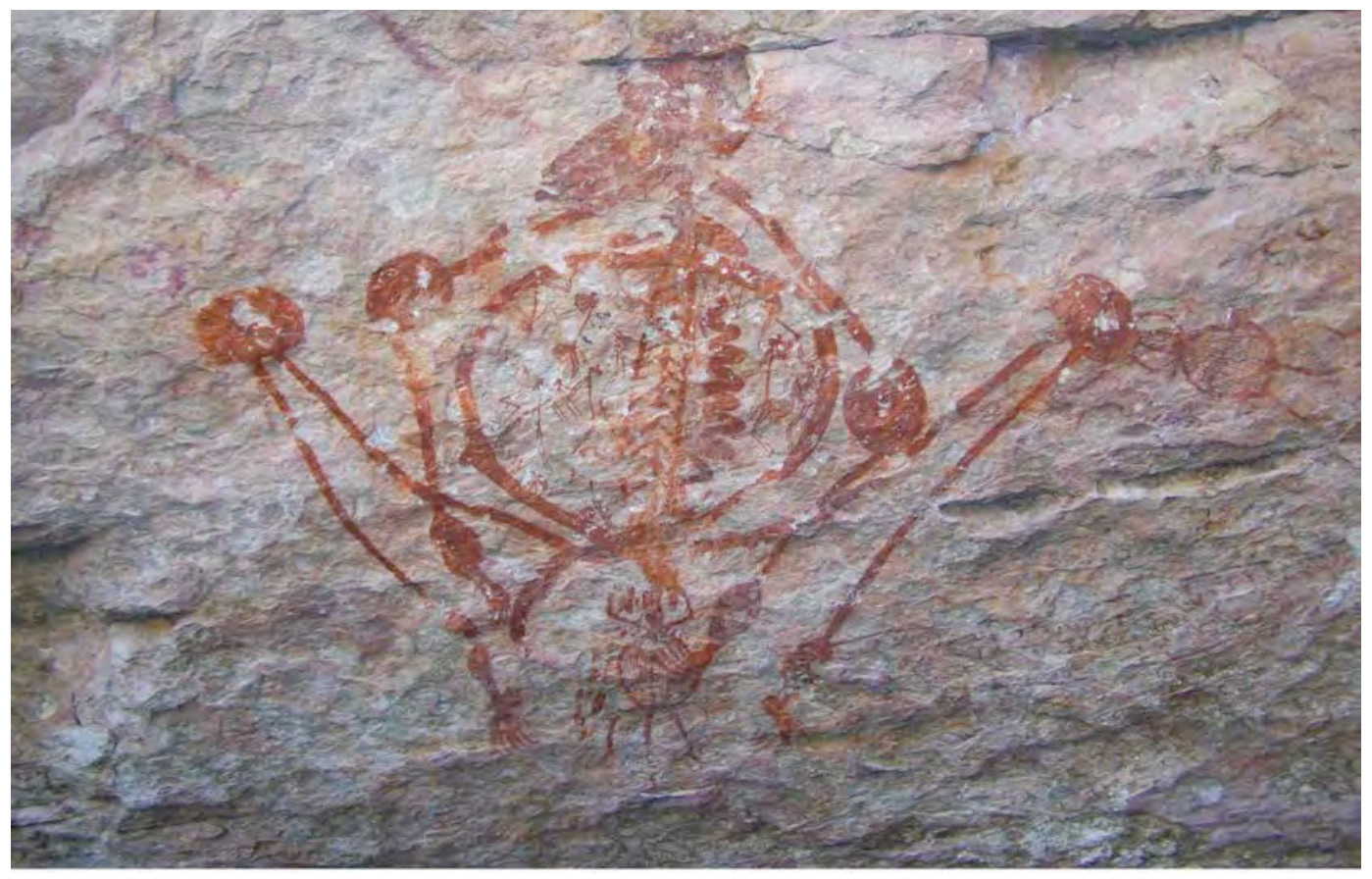


Figure 6 : Representation of fish (Injalak Hill, Gunbalanya). Photograph by I. Domingo.

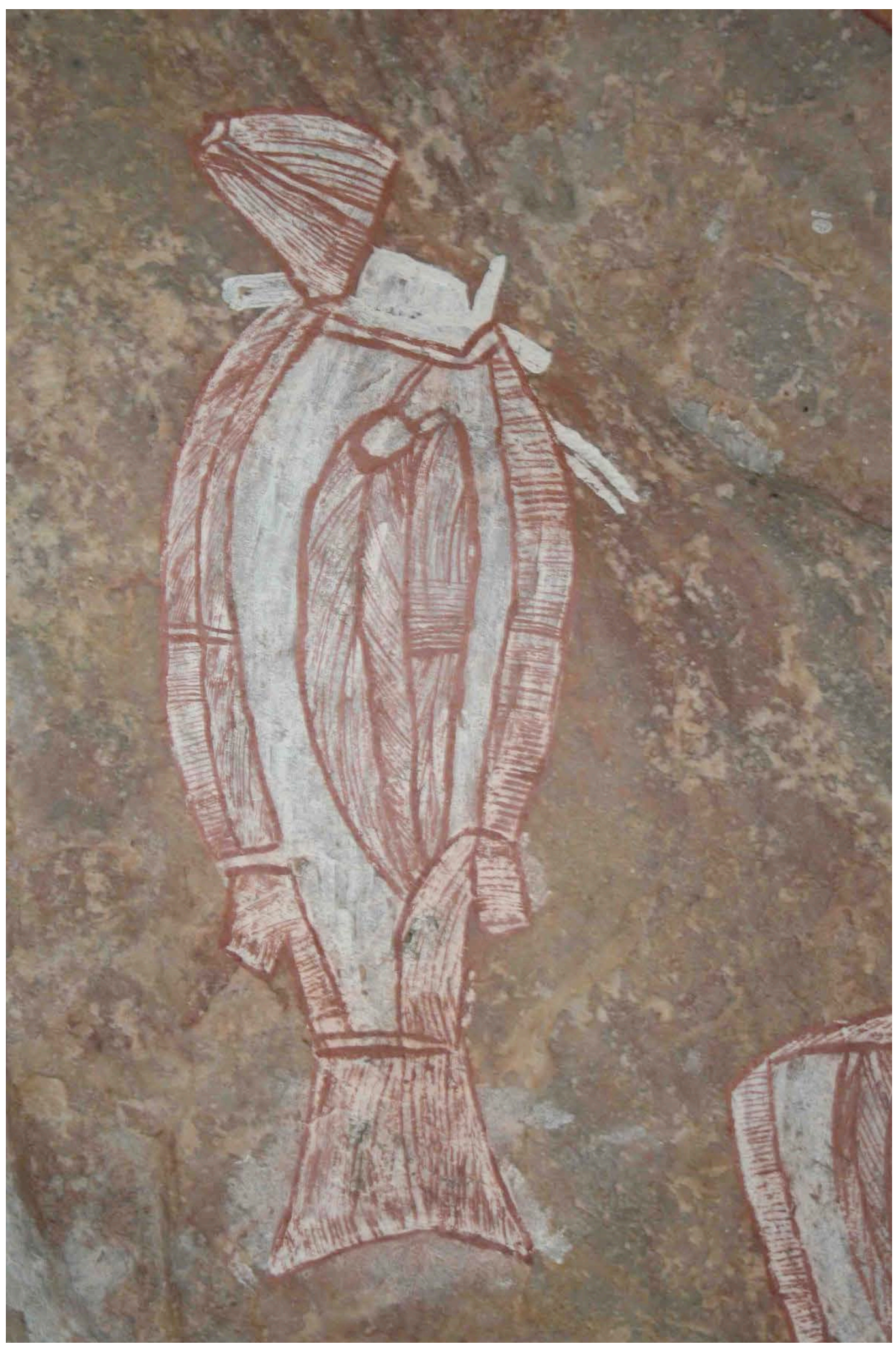


Figure 7 : Water container (Injalak Hill, Gunbalanya). Photograph by I. Domingo.

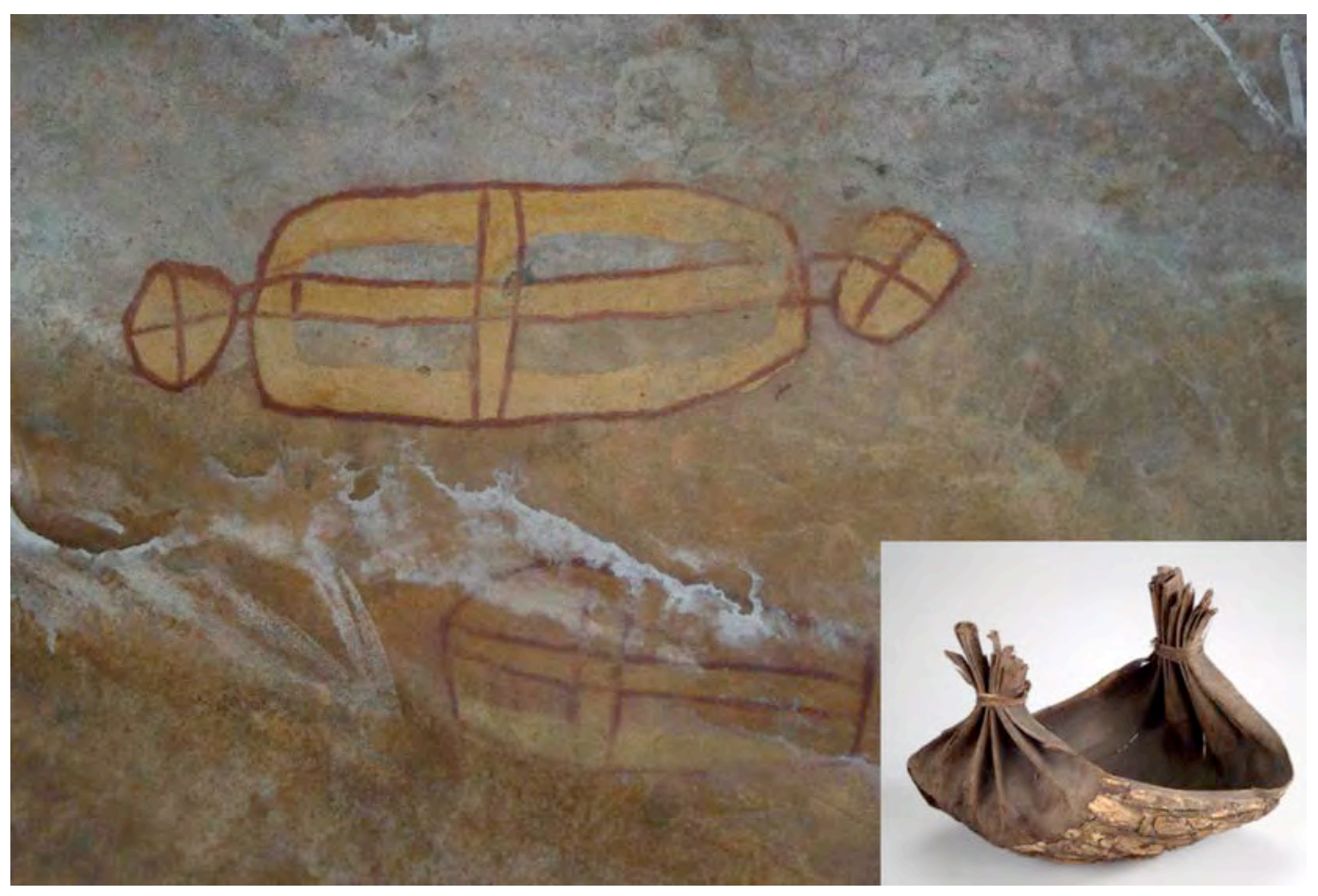


Figure 8: Scene from Djuliri site (Anaru Bay). Photograph and digital tracing by I. Domingo.
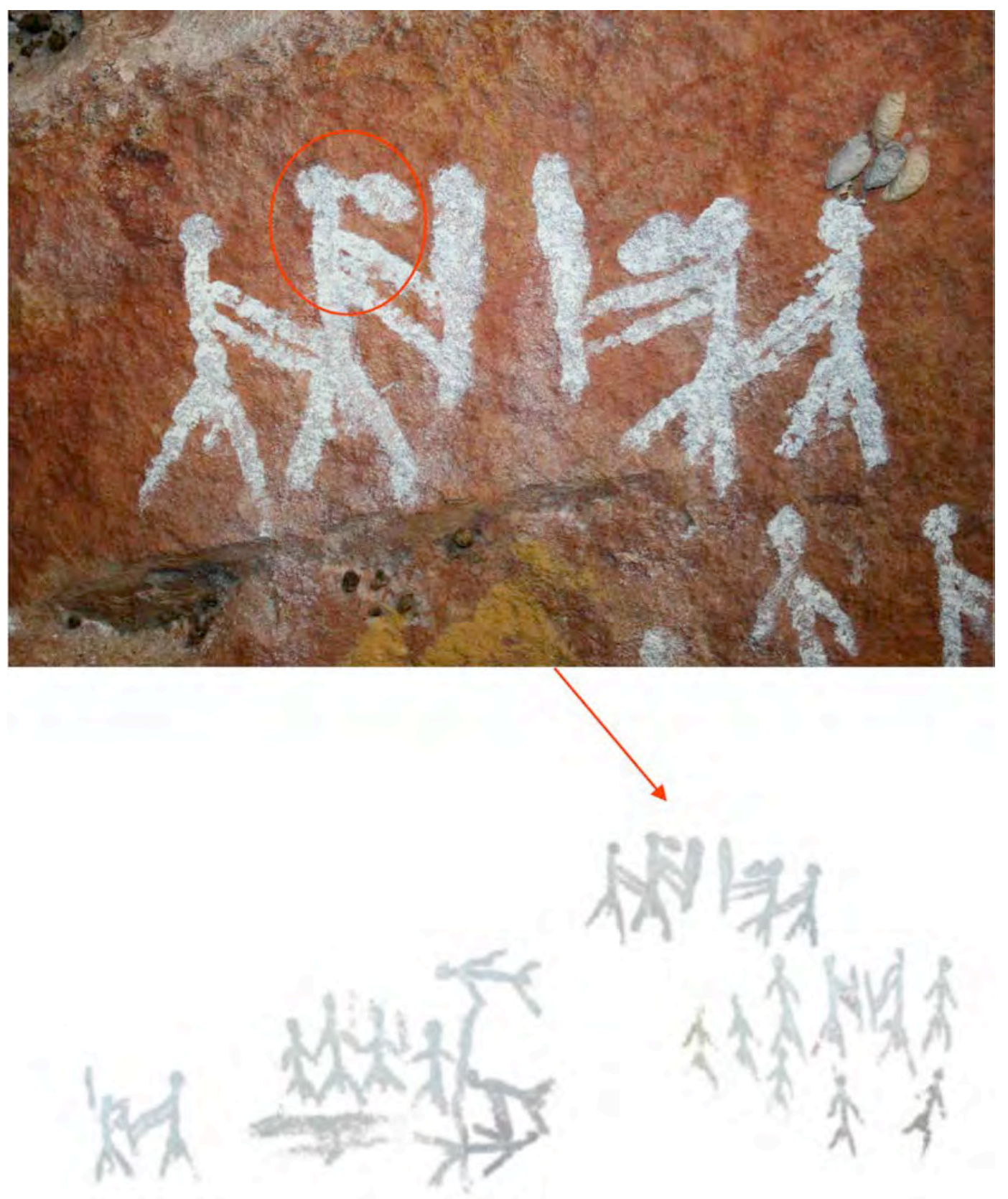
Figure 9: Scene from Djuliri site (Anuru Bay). Photograph and digital tracing by I. Domingo.
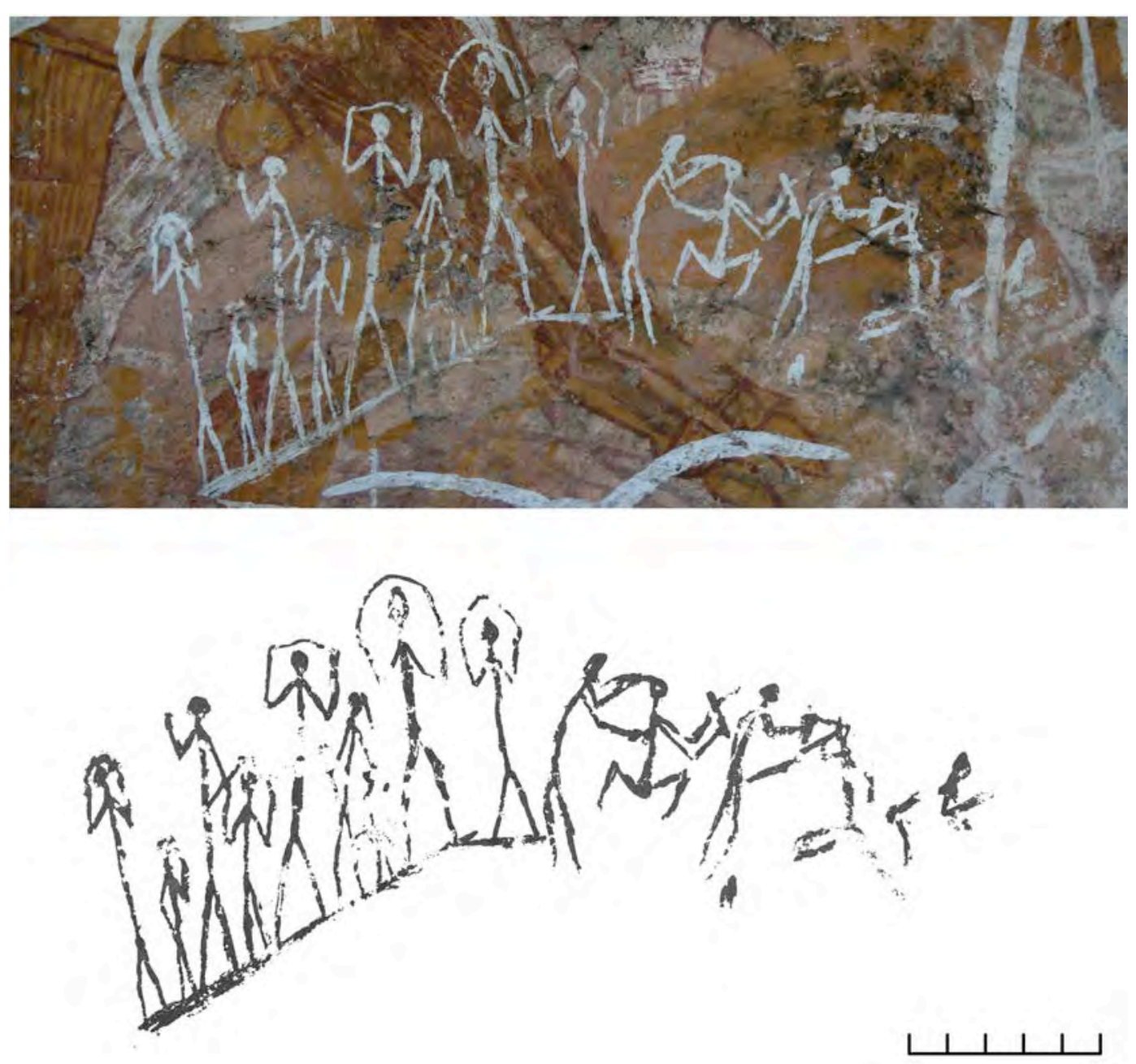
Figure 10 : Mook Mook site (Wugularr). Photograph by I. Domingo.

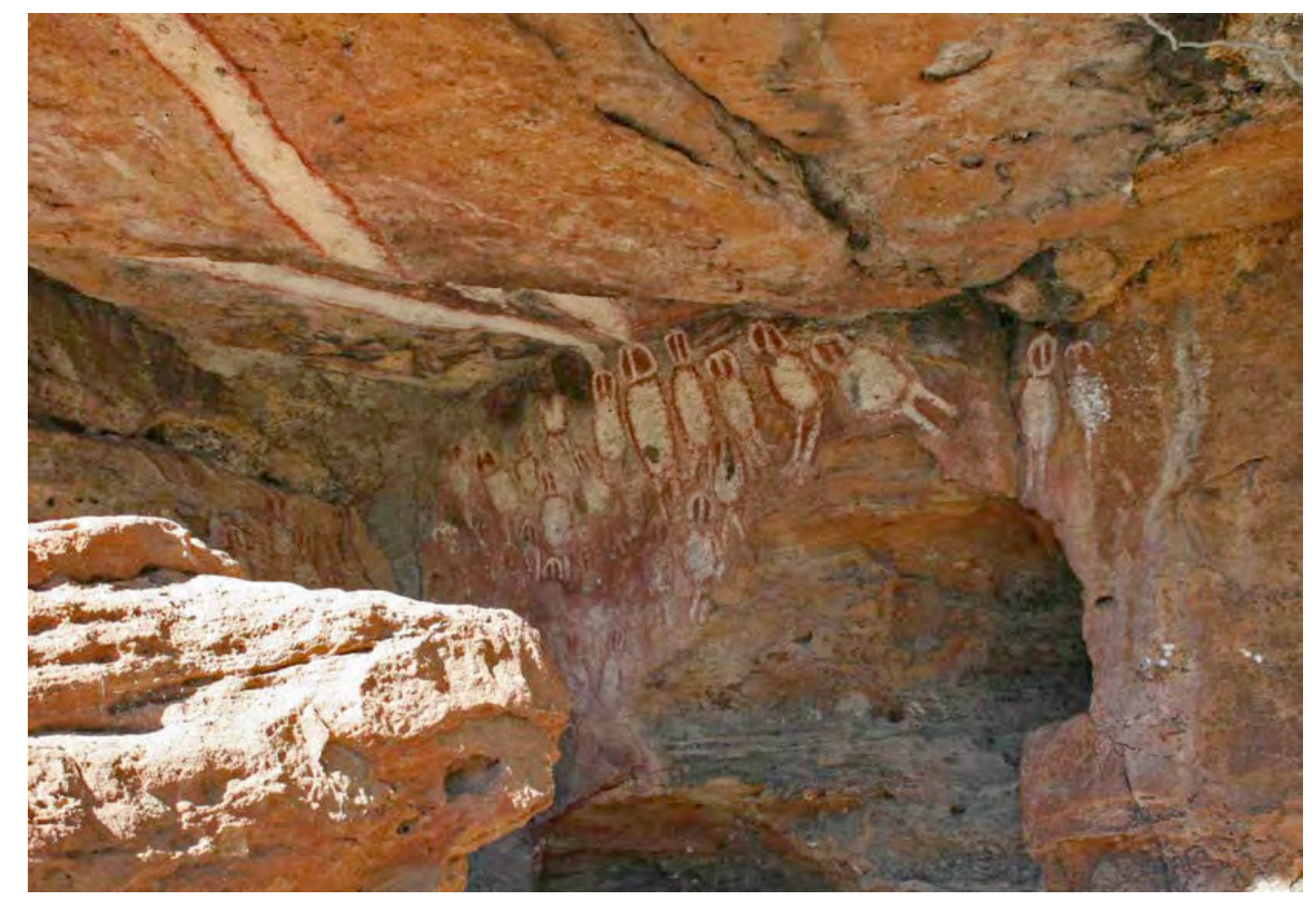




\title{
Langage de signes et communication graphique à la fin du Magdalénien L'art de Rochereil (Grand-Brassac), de l'abri Mège et de la Mairie (Teyjat, Dordogne)
}

\author{
Patrick PAILLET \\ Maître de conférences, Muséum national d'Histoire naturelle, \\ Département de Préhistoire, \\ UMR 7194, Musée de l'Homme
}

Elena MAN-ESTIER

Conservatrice du Patrimoine, Ministère de la Culture et de la Communication, Direction générale des Patrimoines, Service du Patrimoine, Sous-Direction de l'Archéologie UMR 5199 Pacea

Extrait de : Olivier BUCHSENSCHUTZ, Christian JEUNESSE, Claude MORDANT et Denis VIALOU (dir.), Signes et communication dans les civilisations de la parole, Paris, Édition électronique du CTHS (Actes des congrès des sociétés historiques et scientifiques), 2016.

Cet article a été validé par le comité de lecture des Éditions du CTHS dans le cadre de la publication des actes du $139^{\mathrm{e}}$ Congrès national des sociétés historiques et scientifiques tenu à Nîmes en 2014.

\begin{abstract}
Résumé
À partir de quelques exemples tirés de l'iconographie pariétale et mobilière des sites de Rochereil et de Teyjat (La Mairie et l'abri Mège), les auteurs montrent l'originalité et la puissance du langage des signes et de la communication graphique à la fin du Magdalénien, il y a environ 14000 ans cal BP. Cette période est contemporaine des ultimes soubresauts de la dernière glaciation qui induisent une transformation radicale des paysages et des écosystèmes. Les sociétés humaines, jusqu'alors inféodées aux steppes périglaciaires, s'adaptent peu à peu à ces changements en transformant leurs équipements, en révisant leur stratégie économique et cynégétique, en modifiant en quelque sorte leur mode de vie. Elles repensent également le nouveau monde qui les entoure et inventent de nouveaux symboles. La communication graphique et le langage qu'elles soutiennent en sont le meilleur reflet.
\end{abstract}

Mots-clés : Art mobilier, Art pariétal, Magdalénien, Signes, Rochereil, Teyjat

\begin{abstract}
Through some examples of parietal and portable iconography of the sites of Rochereil and Teyjat (La Mairie and Mège shelter), the authors show the power and originality of the symbolic language and graphic communication at the end of Magdalenian, about 14000 years ago cal BP. This period is contemporary of the last back-and-forth of the Late Ice Age that led to an important landscape and ecosystem transformation. Human societies that were directly linked to periglacial steppa are due to an adaptation towards these changes by transforming their weapons, by thinking new economic and hunting strategies, somehow by changing their way of life. They also modify their way of thinking this new world surrounding them and invent new symbols. Their graphic communication with its language is its best reflectance.
\end{abstract}

\section{Un monde en mutation}

Il y a environ 14000 ans cal BP, les dernières sociétés paléolithiques (Magdalénien supérieur et final) évoluent dans des environnements en profonde mutation. L'instabilité climatique qui règne durant le Bölling-Alleröd (GIS-1) a un impact direct sur les écosystèmes. Une recomposition des faunes est alors engagée. Les grands troupeaux des steppes froides et des toundras ouvertes comme l'antilope saïga, le bison et le renne, disparaissent progressivement des paysages. Ils migrent pour la plupart vers le nord, vers l'est ou vers les régions de montagnes. Des espèces animales tempérées comme le 
cerf, l'aurochs ou le sanglier se développent de nouveau et recolonisent des espaces qui se referment progressivement sous l'emprise des forêts (Costamagno, Laroulandie (dir.) 2003). Les derniers magdaléniens exploitent de plus en plus des petits gibiers (léporidés, spermophiles,... ) et domestiquent le loup (Boudadi-Maligne 2010 ; Boudadi-Maligne et al. 2011, 2012, 2014).

Les changements climatiques et environnementaux engendrent une modification de l'économie des groupes préhistoriques. Les hommes adaptent leurs équipements techniques lithiques et osseux. Ils produisent des outils standardisés, notamment sur grandes lames pour les outils domestiques et sur de petites lames ou lamelles pour les instruments de chasse. De nouvelles armes apparaissent, notamment différents types de pointes. On note également des innovations dans les armements en bois de cervidés et dans leur système d'emmanchement. La miniaturisation des équipements de chasse est rendue nécessaire par l'évolution des pratiques cynégétiques en milieu fermé (Langlais 2010, Naudinot 2013, Valentin 2008).

Les pratiques symboliques, notamment le langage et la communication par l'image, évoluent également. Ces changements radicaux revêtent une forte empreinte territoriale ou essaiment plus largement selon une double dynamique qui n'est pas contradictoire dans les paysages en voie de fermeture (Collectif 2014). Les grottes de Rochereil (GrandBrassac) et de la Mairie (Teyjat) dans le nord de la Dordogne sont deux sites majeurs contemporains de cette période de transition entre Magdalénien et Azilien (fig. 1).

\section{La grotte de Rochereil (Grand-Brassac)}

La petite grotte de Rochereil, découverte au début du XX $x^{\mathrm{e}}$ siècle et fouillée essentiellement entre 1937 et 1941, correspond à un lieu d'intenses occupations dont la fonction au Magdalénien était vraisemblablement liée pour partie à la production d'objets ornés et de parures (Man-Estier et Paillet 2013b, Paillet 2014b). En effet, si l'on considère la taille du site (une trentaine de $\mathrm{m}^{2}$ ) et l'épaisseur relativement modeste de la couche magdalénienne (une quarantaine de $\mathrm{cm}$ ), la série d'objets d'art recueillie par le Dr PaulÉmile Jude est considérable (plus de 250 pièces).

La grotte mesure une quinzaine de mètres de longueur et 2 à $3 \mathrm{~m}$ de largeur. Sa hauteur varie d'environ $5 \mathrm{~m}$ à l'entrée à moins de $2 \mathrm{~m}$ dans sa partie la plus profonde. Elle s'ouvre vers le sud-est au pied d'une falaise de calcaire coniacien, sur la rive droite de la Dronne. Les fouilles de P.-E. Jude ont mis au jour d'importants dépôts du Magdalénien et de l'Azilien. Une reprise récente des séries dans le cadre d'un Projet Collectif de Recherche «Peuplements et cultures à la fin du Tardiglaciaire dans le nord du Périgord, entre Dronne et Tardoire» dirigé par P. Paillet a permis de requalifier la séquence archéostratigraphique du site. Il s'agit de Magdalénien supérieur ancien et récent, d'Azilien ancien et récent et de Laborien. P.-E. Jude publie deux coupes stratigraphiques relevées à l'entrée et à l'intérieur de la grotte (Jude et Cruveiller 1938, Jude 1960). La principale, à l'aplomb de l'entrée de la grotte, fait apparaître au moins quatre couches superposées de couleur et de structure différentes:

- la couche I est stérile et repose sur le sol rocheux. Elle est constituée de sables fluviatiles sur la terrasse et d'argile calcaire rougeâtre dans la grotte.

- la couche II correspond au Magdalénien supérieur. Elle mesure près de $40 \mathrm{~cm}$ d'épaisseur. Sur la base de l'hétérogénéité de certains éléments lithiques et faunistiques, cette couche a été partagée arbitrairement en deux niveaux, IIa (niveau inférieur, comprenant les $2 / 3$ de la couche) et IIb (niveau supérieur).

- la couche III correspond à l'Azilien. Elle mesure plus de $1,80 \mathrm{~m}$ d'épaisseur et se superpose directement à la couche II, sans zone stérile intermédiaire. Cette couche est subdivisée en trois niveaux (IIIa, IIIb et IIIc).

- enfin, la couche IV est stérile et constituée de terre végétale. Elle mesure environ $2 \mathrm{~m}$ d'épaisseur. 


\section{La grotte de la Mairie (Teyjat)}

La grotte ornée de la Mairie est située à une vingtaine de $\mathrm{km}$ au nord de Rochereil. Elle s'ouvre au midi, dans le village de Teyjat, au cœur d'un massif de calcaires dolomitiques du Bajocien. Elle est constituée de deux galeries divergentes: une galerie fossile subhorizontale d'une centaine de mètres de longueur, large de $5 \mathrm{~m}$ et haute de $4 \mathrm{~m}$ en moyenne et une galerie active descendante d'une quarantaine de mètres. Le gisement magdalénien et la zone ornée occupent les 10 premiers mètres de la galerie supérieure. Les occupations fouillées au début du siècle par Pierre Bourrinet, aidé épisodiquement par Denis Peyrony et Louis Capitan, ont mis en évidence une importante archéoséquence (jusqu'à $4 \mathrm{~m}$ de remplissage), divisée en deux couches du Magdalénien supérieur (couches inférieures A et B - Magdalénien supérieur ancien et couches supérieures C et D - Magdalénien supérieur récent). Les deux couches ont livré un assemblage lithique et osseux très riche et bien caractéristique du Magdalénien supérieur. Les occupations de la Mairie sont parfaitement synchrones du Magdalénien de Rochereil (Aujoulat 1984; Barrière 1968, 1972 ; Capitan et al. 1908 ; Langlais 2014).

\section{L'abri Mège (Teyjat)}

À environ $200 \mathrm{~m}$ à l'est de la grotte de la Mairie, le petit abri Mège renferme une séquence unique du Magdalénien supérieur ancien, épaisse de $40 \mathrm{~cm}$ à près de $1,60 \mathrm{~m}$ à l'entrée (couche 2). Elle est contemporaine des couches A et B de la Mairie (Capitan et al. 1906, Langlais 2014).

Ces trois sites ont livré d'importantes séries d'objets d'art dont la mise en comparaison est particulièrement enrichissante. Les représentations pariétales de la Mairie, réalisées sur un vieil édifice stalagmitique aujourd'hui disloqué, rappellent par bien des aspects l'art mobilier et notamment celui de Rochereil. Il est donc cohérent de placer l'art pariétal de la Mairie en résonance avec les séries d'objets ornés.

\section{Singularité des arts de la Préhistoire}

L'un des points communs à l'ensemble des sociétés de chasseurs-collecteurs du Paléolithique supérieur est l'existence d'un langage de signes et d'une communication graphique hautement symbolique désignée communément sous le terme $\mathrm{d}^{\prime}$ «art». Présent depuis près de 40000 ans sous sa forme pérenne, l'art préhistorique est remarquable par la persistance de thèmes et de techniques d'expression durant toute sa trajectoire paléolithique. Les évolutions, les changements ou les ruptures qui marquent parfois son développement ne remettent pas radicalement en cause la cohérence de l'ensemble des pratiques artistiques. Elles ne changeront véritablement qu'avec les sociétés agro-pastorales du Néolithique. L'art préhistorique occupe des terrains d'expression différenciés. Les parois, les plafonds et les sols des grottes et des abris, parfois les roches disposées à l'air libre dans des fonds de vallées au cœur de la nature, constituent les supports exclusifs de l'art pariétal et rupestre monumental plus ou moins déconnectés de la vie des hommes. L'art est également présent dans leur quotidien immédiat. Il occupe d'innombrables outils, rehausse une infinité d'armes et se glisse parfois, souvent même, sur des restes fragmentaires sans utilité apparente et immédiate et sur des déchets. La communication graphique paléolithique est élaborée à partir de thématiques fondamentalement ancrées dans l'observation du vivant. L'art préhistorique est souvent qualifié $d^{\prime}$ art animalier et son bestiaire affiche une pluralité zoologique extrême, en particulier dans l'art des objets. Cette diversité est moins grande dans l'art 
des grottes. Cependant, une dizaine d'espèces animales prédominantes constituent la grande majorité de l'iconographie animalière (fig. 2). Il s'agit du cheval, du bison, de l'aurochs, du cerf, de la biche, du renne, du bouquetin, du mammouth, des félins et de l'ours. Les poissons et les oiseaux ne sont pas rares, mais leur dessin résiste souvent à l'interprétation (Citerne 2003, Paillet 2006, Crémadès 1997, Nicolau-Guillaumet 2008).

Les artistes préhistoriques ne puisent pas exclusivement leur source d'inspiration dans le réel et son observation. Le corpus des représentations non-figuratives, parfois qualifiées de géométriques ou plus souvent encore d'abstraites, est immense, plus grand encore que celui des animaux, mais il est plus discret. Les préhistoriens rassemblent sous le vocable de "signes » un corpus polymorphe d'entités graphiques plus ou moins élaborées à partir des formes élémentaires que sont le point, la ligne et le plan (Sauvet 1990). Les signes possèdent des caractéristiques formelles relativement stables auxquelles sont associés conventionnellement un ou plusieurs signifiés. Leur rôle est donc de servir à la communication. C'est aussi le cas de l'image humaine qui est relativement fréquente, mais souvent éloignée de la réalité, soit par le biais de processus de segmentation graphique (mains, membres, sexes isolés, etc.), soit par exagération de traits (bestialisation des profils humains) (Bourrillon et al. 2012, Fuentes 2013, Vialou 1991).

\section{Le réalisme animalier et sa libre expression}

Dans cette "forêt de symboles ", à laquelle il faut ajouter aussi les images totalement réinventées et recréées comme les figures fantastiques ou composites (sorciers, etc.), les magdaléniens (entre 18000 et 14000 ans cal BP), derniers grands chasseurs-collecteurs des steppes et milieux ouverts, se distinguent par l'intensité et la diversité de leurs productions artistiques et par une richesse expressive qui puise son origine dans la méticuleuse observation du réel, mais aussi dans sa libre interprétation (fig. 3). Le réalisme de certaines images magdaléniennes s'enracine dans des traditions anciennes, probablement aurignaciennes, mais il revêt au Magdalénien une dimension du vécu et de l'observé presque unique. Le réalisme s'élabore à la fois par le respect de l'anatomie de l'animal modèle, par la mise en valeur de ses comportements éthologiques et par la propre capacité de la représentation à s'intégrer dans le support (Clottes, Delporte (dir.) 2004, Dubourg 1997).

À Rochereil, un aurochs gravé sur le dos d'un lissoir (fig. 4) constitue un bel exemple de mimésis dans l'expression des formes générales, des détails anatomiques et du rendu des phanères. Par ailleurs, l'animal est représenté couché, dans une position peu commune pour l'iconographie paléolithique, alors qu'elle est assez fréquente dans la réalité. Cette position rappelle le renne accroupi gravé sur l'une des dalles stalagmitiques de la grotte de La Mairie (fig. 5). À Rochereil, les pattes avant sont repliées sous le corps et la corne (unique), fortement cintrée, est projetée vers l'avant. Ces détails offrent à l'animal les conditions nécessaires à l'adaptation de son corps aux contraintes du support.

Mais pour aussi fidèle que soit l'image, elle ne constitue jamais vraiment une transcription parfaite du réel. Au contraire, l'artiste transforme son sujet pour le rendre plus percutant, plus lisible en quelque sorte ou plus vivant paradoxalement. Par le truchement de ces libertés expressives, il donne à l'animal représenté un sens particulier. L'animal ainsi pensé s'éloigne de la stricte réalité. La vue en profil absolu est souvent employée. Elle donne de la bête un contour reconnaissable. Certaines «clés » formelles permettant l'identification, comme l'oreille, la corne ou la forme de la ligne dorsale sont régulièrement mises à profit. Des détails anatomiques, comme le pelage ou les sabots, sont également utilisés par les artistes magdaléniens comme autant d'arguments supplémentaires à la reconnaissance de leur symbole et donc à son partage (Man-Estier et Paillet 2013a). 
L'observation des animaux dans leur milieu naturel est aussi transcrite par la représentation de «scènes » qui peuvent suggérer des moments de leur vie (Paillet et Man-Estier 2013). Deux individus se flairant évoquent irrésistiblement une scène de préaccouplement, mais dans la grotte de la Mairie, l'hypothèse est contrariée par la proposition de l'artiste (fig. 6) : tantôt c'est une énorme femelle bison qui flaire un petit mâle, tantôt le mâle et la femelle aurochs sont dérangés par un troisième individu et par une multitude d'autres représentations animales associées ou superposées à la scène principale (fig. 7).

\section{Des supports d'art originaux : les omoplates}

Au Magdalénien, les supports utilisés par les artistes, tout comme les techniques d'expression mobilisées, sont extrêmement divers. L'art envahit tous les supports, qu'ils soient lithiques ou osseux, ordinaires comme le bois de renne, ou peu communs, voire exceptionnels, comme l'ivoire de mammouth. Parallèlement, on note des tendances de productions qui combinent à la fois un support, une technique et parfois même un thème spécifiques. Il en est ainsi des scapulas (omoplates) gravées et striées finement (Paillet 2014a). On en connaît quelques-unes à la Mairie (fig. 8) et une quarantaine à Rochereil (fig. 9). Ces supports plats et très fragiles sont souvent fragmentaires ; nous n'avons accès qu'à une partie des décors réalisés.

Après une éventuelle préparation des surfaces par d'intenses raclages destinés peut-être à débarrasser le support des tissus mous (chair, graisse), les figures sont gravées, reproduites par tous les types de traits imaginables, larges ou fins, superficiels ou profonds, en raclages, parfois même asymétriques donnant l'illusion du relief exhaussé. Sur ces supports plats, auxquels il convient aussi d'ajouter d'assez nombreuses mandibules et des os coxaux, les techniques de gravure et les thèmes s'entremêlent inextricablement en un apparent désordre. Les animaux (souvent des cervidés ou des chevaux) se croisent, se superposent et s'entremêlent en formant des constructions graphiques presque irréelles, d'authentiques palimpsestes qui résistent souvent à l'analyse et à l'interprétation. Dans l'espace plus ou moins plat, mais contraignant des scapulas, les animaux se logent le plus étroitement possible. Le champ graphique est ainsi optimisé dans toute sa spatialité. Les scapulas gravées, mais aussi peintes dans certains cas (au Gravettien notamment, il y a plus de 26000 ans cal BP), constituent des objets d'art tout à fait exceptionnels durant le Paléolithique. Si leur fonction précise nous échappe, on pressent leur haute valeur symbolique dont la scapulomancie n'est qu'un avatar historique. Elles sont présentes dans de nombreux gisements du grand Sud-Ouest de la France (La Madeleine, Laugerie-Basse, grotte Richard, l'abri du Morin, Isturitz, Masd'Azil) (Capitan et Peyrony 1928 ; Paillet 2014a ; Deffarge et al. 1975 ; Saint-Périer 1930, 1936) et du piémont cantabrique (cueva Oscura, El Cierro, Rascaño, El Juyo, Altamira, El Castillo) (de las Heras et al. 2010-2011). Si leur contexte archéostratigraphique n'est pas toujours précis, elles sont connues de la fin du Solutréen (il y a environ 20000 ans) à la fin du Magdalénien. Ailleurs, notamment en Europe centrale et orientale, et dans des temps plus anciens, durant le Pavlovien ou le Gravettien, ce sont des scapulas peintes qui dominent (Mons et Péan 2014).

\section{Une nouvelle manière de penser et de reproduire le réel}

Les magdaléniens, dans les phases moyennes ou plus récentes de leur développement, ont exploré la réalité dans des directions multiples. Comme nous l'avons vu plus haut, la fidélité au réel n'a jamais constitué une règle stricte. On qualifie à tort les artistes magdaléniens de "naturalistes » parce qu'on ne retient dans le vaste corpus hétéroclite de leurs productions graphiques et plastiques que celles qui donnent l'illusion de 
reproduire objectivement le réel, mais dans les faits, les artistes retravaillent et modèlent la réalité en fonction de leur propre vision du monde et des messages qu'ils souhaitent transmettre (fig. 10). Le réalisme des images magdaléniennes n'est qu'une illusion. Il est parfois même une véritable déconstruction/reconstruction du réel comme l'attestent les formes animales ou humaines stylisées ou schématisées qui se diffusent sur de larges territoires vers la fin du Magdalénien (Bosinski 2011, Bourrillon et al. 2012). Trois exemples iconographiques sont emblématiques de cette nouvelle manière de penser et de traiter les formes, de les codifier graphiquement. Il s'agit des chevaux macrocéphales ou barygnathes, de certains animaux représentés plus ou moins schématiquement en perspective frontale (caprinés et cervidés notamment) et des figures féminines schématiques, les FFS.

\section{Des chevaux à grosses têtes}

Les chevaux macrocéphales forment un corpus très homogène de 176 entités graphiques disposées sur une soixantaine d'objets découverts dans une douzaine de sites du grand Sud-Ouest de la France (Apellaniz 1990, Paillet 2014a). Ce sont des gisements localisés pour l'essentiel en Périgord, dans les vallées de la Dordogne (Le Souci - 16 figures et Limeuil - 1), de la Vézère (Laugerie-Basse - 18, Les Eyzies - 2, La Madeleine - 85 et Jolivet - 3), de la Dronne (Rochereil - 5 et La Peyzie - 2), du Bandiat (La Mairie - 3) et de l'Isle (Raymonden - 9) et en Gironde proche (Le Morin - 20). Les autres figures recensées hors de ce petit territoire ont été découvertes dans le Tarn-et-Garonne (Montastruc - 4 et Fontalès - 5) et en Ariège (Mas d'Azil - 3) (Sonneville-Bordes et Laurent 1986, Welté 2001). Aucune représentation pariétale ne se rapproche des chevaux ainsi stylisés. Ce sont des animaux à grosse tête qui ornent le fût de pointes de sagaies robustes, de pièces biseautées et plus rarement le manche ou la tête de bâtons percés et des os d'oiseaux sans fonction utilitaire apparente. Le cheval macrocéphale est construit selon un schéma conventionnel assez strict, répété et reconnaissable (fig. 11).

C'est un animal hypertrophié ou "hypotrophié » selon la partie du corps que l'on considère et ce n'est pas toujours un cheval : il existe en effet quelques rennes traités dans le même style et les mêmes techniques. La tête occupe au moins un quart de la longueur totale de l'animal, voire plus d'un tiers dans certains cas. Les membres sont toujours inachevés. Les têtes sont anguleuses au niveau du museau et de la joue et présentent un contour polygonal. En règle générale, les naseaux, la bouche, l'œil et le zygomatique sont bien indiqués. Le menton et la joue sont lourds, ce qui leur a valu le qualificatif de barygnathe ou macrognathe. Le corps est étroit et anguleux, la jugulaire est soulignée, les côtes sont parfois indiquées par des incisions obliques le long du dos et la crinière fréquemment dressée en heaume hachuré et/ou séparé de l'encolure par un trait. La queue, longue, étroite et pointue est systématiquement décollée de la fesse. Quelques rares individus portent des traits d'épaule, d'autres des traits qui barrent le ventre et les reins. Les contours de l'animal et ses détails sont presque systématiquement obtenus par des incisions profondes à profil dissymétrique de telle sorte que l'animal apparaît en léger bas-relief. Mais, nous devrions plutôt parler au pluriel car les macrocéphales sont rarement isolés et souvent disposés en file de deux à cinq individus, toujours serrés et jamais affrontés. Ils ne trahissent aucun comportement interprétable et sont étroitement cadrés dans la totalité du champ graphique disponible.

Ces animaux sont pour le moins très typés. La dilatation de l'œil ou de l'arcade orbitaire, la saillie de l'arcade zygomatique, le creux de la gouttière jugulaire et parfois la représentation du gril costal, associés à un silhouettage figé et anguleux, donnent à ces représentations un air d'animaux chétifs, amaigris, malades, voire morts, plus que de poneys ou d'ancêtres de Pottok bien vivants. Pourtant, malgré la schématisation poussée parfois aux limites géométriques, voire abstraites, de l'expression graphique et plastique, on reconnaît dans ces macrocéphales des chevaux bien observés dans l'intimité même de leurs détails anatomiques les plus spécifiques. Là est le paradoxe de l'art stylisé de la fin du Magdalénien qui s'éloigne de l'observation scrupuleuse et "photographique » du vivant tout en se rapprochant en même temps de la vraie nature de l'animal, de son 
essence en quelque sorte. L'originalité stylistique de ces chevaux, leur contexte archéostratigraphique précisément identifié dans le temps et leur répartition territoriale limitée autour du site de production qu'est l'abri de La Madeleine, en font des marqueurs chrono-culturels d'une rare résolution (Feruglio 2014).

Les chevaux macrocéphales de Rochereil (fig. 12), placés sur le fût d'une sagaie et d'un outil intermédiaire, n'échappent pas à la règle conventionnelle détaillée au-dessus. Les trois animaux gravés sur un radius de grand oiseau (fig. 13) et provenant des premières fouilles d'Édouard Perrier du Carne dans la grotte de La Mairie doivent être également ajoutés à ce corpus malgré l'originalité du support (objet apparemment non usuel) (Perrier du Carne 1889).

\section{Des bouquetins, des chamois et des cerfs en alerte}

Les représentations conventionnelles d'animaux en vue frontale du Magdalénien supérieur ont en partage leur dessin systématiquement très schématique et stylisé, exécuté en gravure ou au dessin rouge ou noir (pour les rares œuvres pariétales). Henri Breuil les considérait comme des figures "dégénérées » ou graphiquement "altérées » (Breuil 1905, 1907). Ce sont souvent des caprinés, plus rarement des cervidés, qui sont ainsi représentés selon un point de vue original par rapport à la majorité des autres animaux figurés de profil. La reproduction en vue frontale n'est pas l'exercice graphique le plus facile, $\mathrm{du}$ fait de la mise en perspective et en raccourci du corps et de la tête de l'animal. Pourtant, il a été pratiqué assez fréquemment à la fin du Magdalénien dans l'art mobilier du piémont espagnol basque et cantabrique (près de 70 représentations sur une quarantaine de pièces - essentiellement outils et armes en matières dures d'origine animale - provenant de 22 sites), d'une manière moins intense (encore qu'il conviendrait de réviser entièrement le corpus) dans le grand Sud-Ouest français (La Vache, Gourdan, Lortet, Laugerie-Basse, La Madeleine, Raymonden, Rochereil, La Mairie, Montgaudier) et très exceptionnellement sur les parois des grottes (El Otero, La Lastrilla, Ekain, Niaux, Massat) (fig. 14).

Les animaux vus de face ne sont pas fréquents dans l'iconographie paléolithique. Cette disposition traduit peut-être un mouvement ou mieux encore une attitude comportementale (Barandiaran et Cava 2013). C'est le cas notamment des bouquetins, des chamois et des cerfs en alerte permanente dans la nature et dont le comportement d'attention consiste à fixer la source d'un danger potentiel (chasseur, observateur). Ces animaux offrent ainsi leur corps en position frontale ou semi-frontale, mais ce sont surtout la tête, les cornes (ou les bois pour les cervidés) et les oreilles qui dominent la silhouette. Par le trait et sous l'effet de sa stylisation plus ou moins poussée, l'animal figuré fera l'objet d'importantes variations formelles, du traitement réaliste détaillé à la plus extrême schématisation dépouillée. L'animal peut être ainsi complet ou incomplet, son corps vu de face ou de profil et sa tête plus ou moins détaillée. Mais le modèle général demeure toujours le même, notamment pour les caprinés : les cornes sont traitées en longs tracés courbes divergents et les oreilles sont figurées de part et d'autre de l'encornure par des traits plus courts, également divergents (fig. 15). Quand elle est indiquée la tête est plutôt triangulaire et son dessin se confond ou se prolonge parfois sans interruption avec celui de l'encolure. Cette vision des bouquetins, des chamois, des cerfs et plus rarement des bovinés ou des chevaux, propre aux ultimes magdaléniens $\mathrm{d}^{\prime}$ Europe occidentale, nous interroge. Ces animaux vus de face et plus ou moins schématisés côtoient, parfois sur les mêmes supports, des congénères de profil beaucoup plus classiques et surtout moins cursifs.

Le langage des formes magdaléniennes est résolument diversifié et la communication graphique s'opère dans des directions en apparence contradictoires, mais finalement parallèles: le réalisme et le schématisme. La schématisation extrême de certaines représentations animales, que l'on peut suivre parfois pas à pas depuis leur origine figurative à travers des processus de simplification et d'abréviation des contours puis des détails, conduit à l'émergence de véritables signes aux confins du figuratif et du non 
figuratif. La schématisation des caprinés en vue frontale a peut-être revêtu une fonction particulière dans la pensée symbolique magdalénienne en créant du lien métaphorique ou en dissimulant les symboles (Sauvet 1990). On aura également remarqué la dispersion géographique de ces motifs, plus ample que celle des chevaux «macrocéphales ». Elle nous donne à réfléchir sur la nature des relations entretenues entre les groupes humains à longue distance de part et d'autre des Pyrénées (Cazals et al. 2007).

\section{Une autre vision de la femme}

À partir du Magdalénien supérieur, dans l'art pariétal et l'art mobilier, le corps de la femme est célébré d'une manière particulièrement originale, presque inconnue jusqu'alors. De profil, l'Ève des temps magdaléniens est mince contrairement à ses cousines du Gravettien (Bosinski 2011, Collectif 2011). Penchée vers l'avant, elle a le ventre plutôt plat; son torse est étroit et elle est habituellement acéphale. En revanche, elle est fessue et elle repose sur une épaisse cuisse souvent terminée en pointe. Très rarement, elle est dotée de seins et de bras. De profil, son image se réduit peu à peu à une forme cursive caractérisée par un dos cambré et des fesses proéminentes.

Le style dépouillé des figures féminines schématiques (FFS) est conventionnel (fig. 16). Si les figures féminines ainsi vues de profil apparaissent dans l'art pariétal dès le Gravettien (Cussac, Dordogne et Pech-Merle, Lot), c'est essentiellement à la fin du Magdalénien que leur silhouette se schématise de cette manière (Bourrillon 2009). À l'instar des caprinés croqués en perspective frontale, les artistes recherchent des formes de plus en plus simples et dépouillées, mais malgré cela, l'identité féminine du modèle survit au résumé de courbes et de plans gravés, peints ou sculptés.

Les FFS constituent une innovation graphique et plastique tout autant qu'intellectuelle et symbolique. Elle remporte dans l'Europe du Tardiglaciaire un franc succès si l'on en juge par son immense aire de diffusion, des Asturies à la Pologne, hormis le piémont pyrénéen, à l'exception d'un exemplaire mentionné à Gourdan (Fritz et al. 1993). Elle témoigne de l'intensité des contacts et des relations entre les groupes humains à cette époque et dans les espaces encore très ouverts des steppes magdaléniennes.

Si la dispersion des chevaux macrocéphales indique des mouvements réduits et convergents autour de lieux d'intenses activités culturelles, les FFS se meuvent dans des espaces démesurés où les mouvements sociaux, culturels et symboliques divergent dans presque toutes les directions. Elles constituent également à leur manière un marqueur graphique à forte valeur chronologique et culturelle.

On fera abstraction des quatre plaquettes calcaires gravées de plusieurs figures simplifiées, prétendument découvertes dans la grotte de La Mairie, et qui sont vraisemblablement des faux (Duhard 1991), pour n'évoquer ici que les huit représentations gravées de Rochereil dont certaines ont pour singularité d'être vues de trois-quarts ou de face (Paillet 2011) (fig. 17).

\section{Un nouveau système de signes à la fin du Magdalénien}

À travers ces trois exemples thématiques et formels, on perçoit les liens qui unissent les sites de Rochereil et ceux de Teyjat (La Mairie et Mège), ainsi que leurs spécificités, dans le monde magdalénien peut-être en crise, assurément en cours de mutation. On ignore encore si toutes ces manifestations artistiques, dans leur disparité la plus radicale, sont le fruit d'une société humaine unique, homogène en quelque sorte, ou s'il s'agit plutôt de produits issus d'une mosaïque de groupes qui auraient en partage un fond commun de techniques, de symboles et de croyances. Cette dernière hypothèse, privilégiée au sein de la communauté scientifique, trouve un bon argument à Rochereil dans certaines productions graphiques pour le moins originales. Le corpus d'art mobilier de Rochereil, 
sur tous types de supports, rassemble plus de 250 objets. C'est une série très importante, compte tenu de la modeste taille du gisement. Rochereil est à l'évidence un site de production d'œuvres d'art (et de parures) au Magdalénien supérieur, au même titre que la grotte de La Vache (Ariège), La Madeleine ou Laugerie-Basse (Dordogne), La Marche (Vienne) et Gönnersdorf (Rhénanie) (Clottes et Delporte (dir.) 2004 ; Capitan, Peyrony 1928 ; Pales et Tassin-de-Saint-Péreuse 1969, 1976, 1981, 1989 ; Bosinski et al. 2001).

Les objets ornés de motifs exclusivement non figuratifs ou géométriques sont largement majoritaires (près de 200). Nous n'avons recensé que 25 pièces à décor figuratif et une trentaine associant géométrique et figuratif. Une étude approfondie des décors dits «abstraits » ou géométriques doit être entreprise, mais une première analyse nous permet déjà d'isoler des catégories morphologiques récurrentes qui présentent une certaine stabilité formelle, malgré quelques variantes, et qui s'apparentent donc à de vrais « signes » dans le sens sémiologique du terme, mais ils échappent souvent à la méthode de classement typologique et ils résistent à la terminologie. En d'autres termes, nous avons le plus grand mal à les décrire et à les nommer. Ce sont notamment des motifs ou des signes qui ont en commun les mêmes caractéristiques formelles : ils font penser à des représentations végétales. Ce sont des fuseaux, des ogives ou des ellipses, prolongés d'un trait et dotés de remplissages linéaires internes qui rappellent le limbe d'une feuille avec ses nervures, principale et secondaires, et son pétiole (fig. 18). Ce sont aussi des motifs ovales plus complexes couronnés par deux tracés courbes orientés vers l'extérieur (deux feuilles linéaires ?) et disposés en symétrie. L'origine figurative de ces motifs est prégnante, mais elle est confuse. Du végétal ou de l'animal lequel est le meilleur prétendant? Henri Breuil, qui fut l'un des premiers à s'intéresser à ces figures qu'il qualifiait de "dégénérées », entretint le doute. Tout à la fois, il parlait d'évolution phytomorphique du motif (1907, p. 6), en employant un vocabulaire descriptif particulièrement fleuri (pédoncule, fleur, lys, tubercule, rave, etc.), et prétendait qu'il s'agissait d'abréviations d'animaux vus de face et notamment des têtes de caprinés ou d'équidés (Breuil 1905, 1907). La quête de l'origine figurative des signes élaborés n'a finalement pas beaucoup de sens. En effet, si nous ne disposons pas de toutes les étapes graphiques des processus de schématisation, comme c'est le cas d'une certaine manière des FFS, nous courons le risque de nous enfermer dans des impasses interprétatives. Le principal enseignement que nous pouvons tirer de cette première révision des motifs élaborés de Rochereil, c'est l'extrême complexité des processus de schématisation mis en œuvre par les artistes magdaléniens. Le glissement graphique progressif de certaines images figuratives vers une « ornementation " géométrique, la tendance au schématisme sur les armes, les outils ou d'autres supports en matières dures animales est une constante dans l'iconographie mobilière du Magdalénien, particulièrement dans sa phase finale (motifs pisciformes, etc.). Les formes épurées facilitent vraisemblablement la polyvalence sémantique des signes. En même temps, l'interprétation et la compréhension de ces formes de plus en plus abstraites se réduisent à proportion du groupe ou des individus qui les ont créées.

Les figurations cursives évoquées ici nous montrent que le langage des signes et la communication graphique au Magdalénien s'exerçaient dans de multiples directions et sous différentes formes (polymorphie), parfois très abstraites, comprises par un groupe, tantôt largement transmises et tantôt réservées. Ces symboles, qui constituent l'un des ensembles abstraits les plus homogènes et les plus originaux de l'art paléolithique, forment un langage socialement normé, riche des concepts idéologiques de la société qui les a créés et de la propre expression de l'individu qui les a matérialisés par l'image. Ces productions sont spécifiques presque exclusivement au site de Rochereil. De rares motifs similaires sont connus dans d'autres sites, comme à La Madeleine, à Laugerie-Basse ou à Teyjat (fig. 19), mais de manière isolée ou ponctuelle. Ils témoignent s'il en était besoin de l'intensité de la vie spirituelle durant la Préhistoire, de l'originalité des langages symboliques et de la puissance des échanges entretenus entre les groupes magdaléniens sur les vastes territoires progressivement transformés par les changements climatiques de la fin du Tardiglaciaire. 


\section{Bibliographie}

APELlaniz J.-M. (1990). Modèle d'analyse d'une école dans l'iconographie mobilière paléolithique: l'école des graveurs de chevaux hypertrophiés de La Madeleine, in J. ClotTes (dir.), L'art des objets au Paléolithique, les voies de la recherche, Actes des colloques de la Direction du Patrimoine, Colloque de Foix-Mas d'Azil, 16-21 novembre 1987, t.2, Ministère de la Culture, p. 105-138.

Aujoulat N. (1984). Grotte de la Mairie, in L'art des cavernes, atlas des grottes ornées paléolithiques françaises, Ministère de la Culture, Paris, p. 232-235.

BARANDIARAN I. (de) et CAVA A. (2013). La cabra alerta: marcador gráfico del Magdaleniense cantábrico avanzado, in M. RAsilla (de) (dir.) F.J. Fortea Pérez Universitatis Ovetensis Magister. Estudios en Homenaje, Universidad de Oviedo, Ménsula Ediciones, Oviedo, p. 263-286.

BARRIÈRE C. (1968). Les gravures de la grotte de la Mairie à Teyjat, Travaux de l'Institut d'Art préhistorique, Toulouse, T. X, p.1-13.

BARRIÈRE C. (1972). Grotte de la Mairie à Teyjat, une nouvelle petite gravure, Travaux de l'Institut d'Art préhistorique, Toulouse, T. XIV, p. 204-205.

BOSINSKI G. (2011). Femmes sans tête. Une icône culturelle dans l'Europe de la fin de l'époque glaciaire, Collection Pierres tatouées, Éditions Errance, Arles, 232 p.

BOSINSKI G., D'ERRICO F. et SCHILlER P. (2001). Die gravierten Frauendarstellungen von Gönnersdorf, Franz Steiner Verlag, Stuttgart, 367 p.

BOUdADI-MALigne M. (2010). Les Canis pléistocènes du Sud de la France: approche biosystématique, évolutive et biochronologique, Université Bordeaux 1, PACEA, UMR 5199, Thèse de doctorat, $451 \mathrm{p}$.

BoudADI-MALigne M. et MALlye J.-B. (2011). New data about wolf domestication during the upper Palaeolithic and implications for human groups, Hominid-Carnivore interactions during the Pleistocene, International Congress, Salou, Espagne.

Boudadi-Maligne M., Mallye J.-B., LANGlais M. et BARShay-Szmidt C. (2012). Des restes de chiens magdaléniens à l'abri du Morin (Gironde, France). Implications socioéconomiques d'une innovation zootechnique, Paléo, n²3, p. 39-53.

BOUdADI-MALIGNE M., EsCARGUEL G. (2014). A biometric re-evaluation of recent claims for Early Upper Palaeolithic wolf domestication in Eurasia, Journal of Archaeological Science 45, 80-89.

BOURRILLON R. (2009). Les représentations féminines dans l'Europe du Paléolithique supérieur: une vision diachronique, Préhistoire, Art et Sociétés, Bulletin de la Société préhistorique Ariège-Pyrénées, T. LXIV, p.105-115.

BourRILlON R., Fritz C. et SAUvet G. (2012). La thématique féminine au cours du Paléolithique supérieur européen, permanences et variations formelles, Bulletin de la Société préhistorique française, t.109, nº 1 , p. 85-103.

BREUIL H. (1905). La dégénérescence des figures d'animaux en motifs ornementaux à l'époque du renne, Comptes rendus des séances de l'Académie des Inscriptions et Belles Lettres, $49^{\mathrm{e}}$ année, $n^{\circ} 1$, p. 105-120. 
BREUIL H. (1907). Exemples de figures dégénérées et stylisées à l'époque du renne, Comptes rendus du XIII ème Congrès international d'Anthropologie et d'Archéologie préhistorique, Monaco 1906, p. 394-403.

CapitAn L., Breuil H., Bourrinet P. et Peyrony D. (1906). L'abri Mège. Une station magdalénienne à Teyjat (Dordogne), Revue de l'École d'Anthropologie, $16^{\text {eme }}$ année, T. VI, p. 196-212.

CAPItAn L., Breuil H., Bourrinet P. et PEyrony D. (1908). La grotte de la Mairie à Teyjat (Dordogne). Fouilles d'un gisement magdalénien, Revue de l'École d'Anthropologie, $18^{\text {ème }}$ année, T. V, p. 153-173 et p. 198-218.

CAPITAN L. et PEYRONY D. (1928). La Madeleine, son gisement, son industrie, ses auvres d'art. Librairie Émile Nourry, Paris, 125 p.

CAZAls N., GONZALEZ URQUiJO J. et TERRADAS X. (Eds.) (2007). Frontières naturelles et frontières culturelles dans les Pyrénées préhistoriques / Fronteras naturales y fronteras culturales en les Pirineos prehistóricos, PubliCan-Ediciones de la Universidad de Cantabria.

Citerne P. (2003). Les poissons dans l'art paléolithique: un thème figuratif au cour de l'iconographie magdalénienne, Thèse de Doctorat en Préhistoire, Université de Toulouse-Le Mirail, 476 p.

Clottes J. et Delporte H. (dir.) (2004). La grotte de la Vache (Ariège). Fouilles Romain Robert. Collection Documents Préhistoriques, n¹6, Co-édition CTHS-RMN, 2 volumes.

Collectif (2011). Mille et une femmes de la fin des temps glaciaires, Catalogue d'exposition, Musée national de Préhistoire, Les Eyzies-de-Tayac, 17 juin-19 septembre 2011, Réunion des Musées Nationaux, Paris, 137 p.

Collectif (2014). Grands sites d'art magdalénien. La Madeleine et Laugerie-Basse, il y a 15000 ans, Catalogue d'exposition, Musée national de Préhistoire, Les Eyzies-de-Tayac et Musée d'Art et d'Archéologie du Périgord, Périgueux, 21 juin-10 novembre 2014, Réunion des Musées Nationaux, Paris, 127 p.

Costamagno S. et LAROUlAndiE V. dir. (2003). Mode de vie au Magdalénien: apport de l'archéozoologie / Zooarchaeological insights into Magdalenian lifeways, Actes du colloque 6.4 du XIV ${ }^{\mathrm{e}}$ Congrès de l'UISPP, Liège, Belgique, 2-8 septembre 2001. BAR international serie $1144,138 \mathrm{p}$.

Costamagno S., LAROUlandie V., LANGlais M. et Cochard D. (2009). Exploitation du monde animal sur le versant nord des Pyrénées durant le Tardiglaciaire, in FULLOLA J.M., VALDEYRON N. et LANGLAIS M. (Eds.) Els Pirineus i les àrees circumdants durant el Tardiglacial. Mutacions $i$ filiacions tecnoculturals evolucion paleoambiental, Actes du XIV Colloque international d'Archéologie de Puigcerdà, Hommage à G. Laplace. Institut d'Estudis Ceretan, p. 185-209.

CRÉMADÈS M. (1997). Nouvelles figurations d'oiseaux de l'art mobilier paléolithique franco-espagnol, Paléo, n9, p. 371-387.

DefFarge R, LAurent P. et SOnNeville-Bordes D. de (1975). Art mobilier du Magdalénien supérieur de l'abri Morin à Pessac-sur-Dordogne (Gironde), GalliaPréhistoire, T. 18, n¹, p.1-64.

DUBOURG C. (1997). Les expressions du naturalisme dans les arts graphiques du Paléolithique supérieur. Une vision du monde des chasseurs préhistoriques, Thèse de Doctorat, Université Bordeaux I. 
DUHARD J.-P. (1991). À propos de gravures féminines sur plaquettes calcaires prétendues de Teyjat et supposées magdaléniennes, Paléo, n³, p. 131-137.

FERUGLIO V. (2014). L'art mobilier magdalénien de la région des Eyzies : sur la piste des liens sociaux en Europe occidentale, Grands sites d'art magdalénien. La Madeleine et Laugerie-Basse, il y a 15000 ans, Catalogue d'exposition MNP-MAAP, éditions RMN, p. 68-71.

Fritz C., TOSEllo G. et PINÇON G. (1993). Les gravures pariétales de la grotte de Gourdan (Gourdan-Polignan, Haute-Garonne), Pyrénées préhistoriques, arts et sociétés, Actes du $118^{\text {ème }}$ Congrès national des Sociétés historiques et scientifiques, Pau, CTHS, Paris, p. 381402 .

FuENTES O. (2013). The depiction of the individual in Prehistory : human representation in Magdalenian societies, Antiquity, vol. 87, n 228, p. 985-1000.

Heras C. de las, Lasheras J.-A., Rasines P., Montes R. Fatás P., Prada A. et Muños E. (2010). Datation et contexte archéologique de la nouvelle omoplate gravée découverte à Altamira, in ClOTTES J. (dir.) 2012, L'art pléistocène dans le monde / Pleistocene art of the world / Arte pleistoceno en el mundo, Actes du Congrès IFRAO, Tarascon-sur-Ariège, septembre 2010 - Symposium "Art mobilier pléistocène ", n spécial de Préhistoire, Art et Sociétés, Bulletin de la Société Préhistorique Ariège-Pyrénées, LXV-LXVI, 2010-2011, CD : p. 1571-1588.

Jude P.-E. et CRUVEILLER J. (1938). La grotte et l'homme de Rochereil, Bulletin de la Société historique et archéologique du Périgord, T. LXV, p. 190-197.

JUDE P.-E. (1960). La Grotte de Rochereil. Station magdalénienne et azilienne, Archives de l'Institut de Paléontologie Humaine, Mémoire n³0, Paris, 74 p.

Kuntz D. et Costamagno S. (2011). Relationships between reindeer and man in southwestern France during the Magdalenian, Quaternary International, 238, (1-2), p. 1224.

LANGLAIS M. (2010). Les sociétés magdaléniennes de l'isthme pyrénéen, éditions du CTHS, Paris, $336 \mathrm{p}$.

LANGLAIS M. (2014). Le Magdalénien supérieur de Teyjat (Dordogne). Complément d'informations à partir de l'examen des collections du Musée d'Archéologie nationale, in P. PAillet (dir.) Peuplements et cultures à la fin $d u$ Tardiglaciaire dans le nord du Périgord, entre Dronne et Tardoire, Rapport d'opération archéologique «Projet Collectif de Recherche - 2012-63», 17 p.

Langlais M., Costamagno S., Laroulandie V., Pétillon J.-M., DisCamps E., Mallye J.B., COCHARD D. et KUNTZ D. (2012). The evolution of Magdalenian societies in SouthWest France between 18,000 and 14,000 calBP : Changing environments, changing tool kits, Quaternary International 272/273, p. 138-149.

Langlais M., Laroulandie V., PÉtillon J.-M., Mallye J.-B. et Costamagno S. (2014). Évolution des sociétés magdaléniennes dans le sud-ouest de la France entre 18000 et 14000 calBP : recomposition des environnements, reconfiguration des équipements, in Transitions, ruptures et continuité en Préhistoire, Actes du Congrès préhistorique de France, Bordeaux, Les Eyzies 31 mai-05 juin 2010, Société préhistorique française, Paris, p. $417-430$.

LAROULANDIE V. (2000). Taphonomie et archéozoologie des oiseaux en grotte : applications aux sites paléolithiques du Bois-Ragot (Vienne), de Combe Saunière (Dordogne) et de la Vache (Ariège), Thèse de Doctorat, Université de Bordeaux I. 
LARTET E. et CHRISTY H. (1865-1875), Reliquiae Aquitanicae, being contributions to the archaeology and Palaeontology of Perigord and the adjoining provinces of Southern France, Londres, Williams and Norgate (H. Baillière), 1865-1875, In-8 , 506 p.

MAN-Estier E. et PAILLET P. (2013a). Réflexions sur le réalisme et le naturalisme dans l'art préhistorique. L'exemple de l'ours, in M. GROENEN (dir.) Expressions esthétiques et comportements techniques au Paléolithique, Actes des sessions thématiques 36 et 37, XVI ${ }^{\text {ème }}$ congrès UISPP- SAB, 4-10 septembre 2011, Florianópolis, Brésil, BAR International Series, 2496, vol.3, Archaeopress, Oxford, p. 73-86.

MAN-Estier E. et PAILlet P. (2013b). Rochereil et l'art magdalénien de la fin du Tardiglaciaire dans le nord du Périgord (Dordogne, France), in M. GROENEN M. (dir.) Expressions esthétiques et comportements techniques au Paléolithique, Actes des sessions thématiques 36 et 37, XVI ${ }^{\text {ème }}$ congrès UISPP-SAB, 4-10 septembre 2011, Florianópolis, Brésil), BAR International Series, 2496, vol.3, Archaeopress, Oxford, p. 7-36.

MONS L. et PÉAN S. (2014). Fiche scapula/omoplate, Commission de nomenclature sur l'industrie de l'os préhistorique. Fiches typologiques de l'industrie osseuse préhistorique, Cahier XIII, Matières d'Art, Représentations préhistoriques et supports osseux relations et contraintes, Éditions Errance, p. 143-174.

NAudinot N. (2013). La fin du Tardiglaciaire dans le Grand-Ouest de la France, Bulletin de la Société préhistorique française, T. 110, 2, p.233-255.

Nicolau-Guillaumet P. (2008). Avifaune et art paléolithiques. Essai pour une bibliographie exhaustive, ALAUDA, 76.4, p. 287-298.

PAillet P. (2006). Les arts préhistoriques, Collection Histoire, éditions Ouest-France, 127 p.

PAILlET P. (2011). Les figurations féminines de la grotte de Rochereil, in Mille et une femmes de la fin des temps glaciaires, Catalogue d'exposition, Musée national de Préhistoire, Les Eyzies-de-Tayac, 17 juin-19 septembre 2011, Réunion des Musées nationaux, Paris, p. 118-119.

PAILlET P. (2014a). L'art des objets de la Préhistoire. Laugerie-Basse et la collection du marquis Paul de Vibraye au Muséum national d'Histoire naturelle, Editions Errance, Arles, 2014, 216 p.

PAillet P. (2014b). Men and cultures at the end of the Late Ice Age in the north of Perigord: continuities and ruptures, in Bansi Lal Malla (ed.), Rock Art Studies, Vol. 1, Concept, Methodology, Context, Documentation and Conservation, International Conference on Rock Art, 06-13 December 2012, Understanding Rock Art in Context, IGNCA Series - 10, Indira Gandhi National Centre for the Arts, New Delhi, Aryan Books International, p. $135-150$.

PAILlET P. et MAN-ESTIER E. (2013). Du vivant à sa représentation. Les animaux gravés de la grotte de la Mairie à Teyjat, Les Dossiers d'Archéologie, n³58, p. 22-27.

PALES L. et TASSIN-DE-SAINT-PÉREUSE M. (1969). Les gravures de la Marche. I - Félins et ours, suivi du félin gravé de la Bouiche. Mémoire $\mathrm{n}^{\circ} 7$ des Publications de 1'Institut de Préhistoire de l'Université de Bordeaux, Delmas, 272 p.

PALES L. et TASSIN-DE-SAINT-PÉREUSE M. (1976). Les gravures de la Marche. II - Les humains. Ophrys éditions, Gap et Paris, 178 p.

PALES L. et TASSIN-DE-SAINT-PÉREUSE M. (1981). Les gravures de la Marche. III - Équidés et Bovidés. Ophrys éditions, Gap et Paris, 145 p. 
PAles L. et TASSIN-DE-SAINT-PÉREUSE M. (1989). Les gravures de la Marche. IV - Cervidés, mammouths et divers. Ophrys éditions, Gap et Paris, $121 \mathrm{p}$.

PERRIER du CARNE E. (1889). La grotte de Teyjat, gravures magdaléniennes, éditions C. Reinwald, Paris, 17 p.

SAINT-PÉRIER R. de (1930). La Grotte d'Isturitz. I : le Magdalénien de la Salle de Saint-Martin. Paris, Archives de l'Institut de Paléontologie Humaine, Masson, Paris.

SAINT-PÉRIER R. de (1936). La Grotte d'Isturitz. II : le Magdalénien de la Grande Salle. Paris, Archives de l'Institut de Paléontologie Humaine, Masson, Paris.

SAUvet G. (1990). Les signes dans l'art mobilier, in J. ClotTES (dir.) L'art des objets au Paléolithique, les voies de la recherche, Actes des colloques de la Direction du Patrimoine, Colloque de Foix-Mas d'Azil, 16-21 novembre 1987, t.2, Ministère de la Culture, p. 83-99.

SONNEVILLE-BORDES D. (de) et LAURENT P. (1986). Figurations de chevaux à l'abri Morin : observations complémentaires, Antiquités nationales, n¹8/19, p. 69-74.

VALENTIN B. (2008). Jalons pour une paléohistoire des derniers chasseurs (XIVe-VI millénaire avant J.-C.), Publications de La Sorbonne, Paris, 325 p.

Vialou D. (1991). La Préhistoire. Collection l'Univers des Formes, éditions Gallimard, Paris, $433 \mathrm{p}$.

Welté A.-C. (2001). L'art mobilier magdalénien de Fontalès (Tarn-et-Garonne) dans la vallée de l'Aveyron: les représentations anthropomorphes et zoomorphes, Thèse de doctorat d'État en Préhistoire-Archéologie, Université de Besançon, 5 volumes. 


\section{Illustrations}

Figure 1 : Carte de situation des sites de Rochereil et Teyjat. DAO Patrick Paillet.

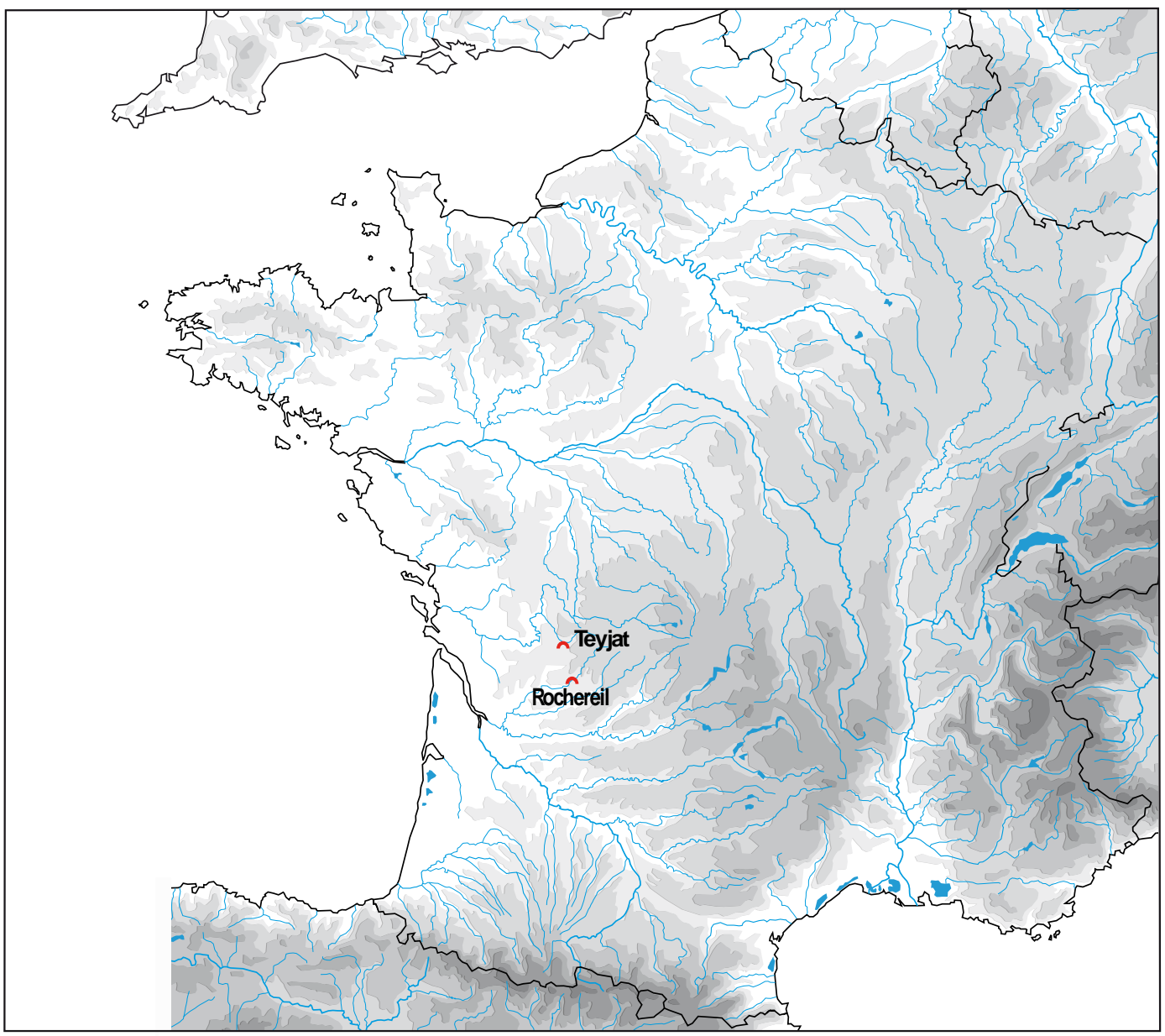


Figure 2 : Bestiaire de la grotte de La Mairie (Teyjat). a : aurochs du panneau $\mathrm{F} ; \mathrm{b}:$ bison du panneau D ; c : cerf du panneau B ; d : biche du panneau B ; e : ours du panneau A ; f : renne du panneau E ; g : cheval du panneau D. Relevés sélectifs Patrick Paillet.
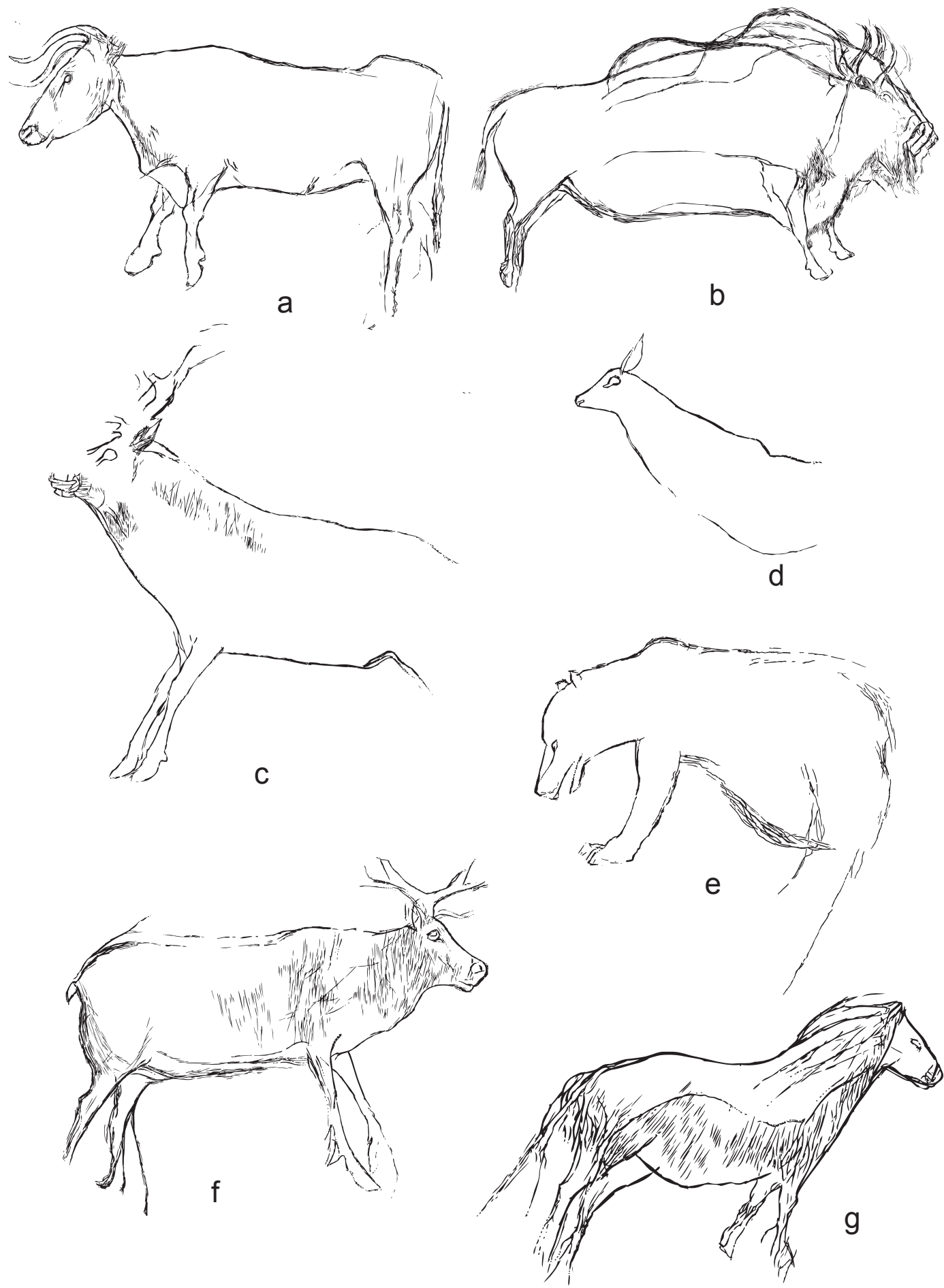
Figure 3: Figures énigmatiques (fantastiques ou composites) gravées sur lissoir, Rochereil (Musée national de Préhistoire). Cliché Émilie Lesvignes.

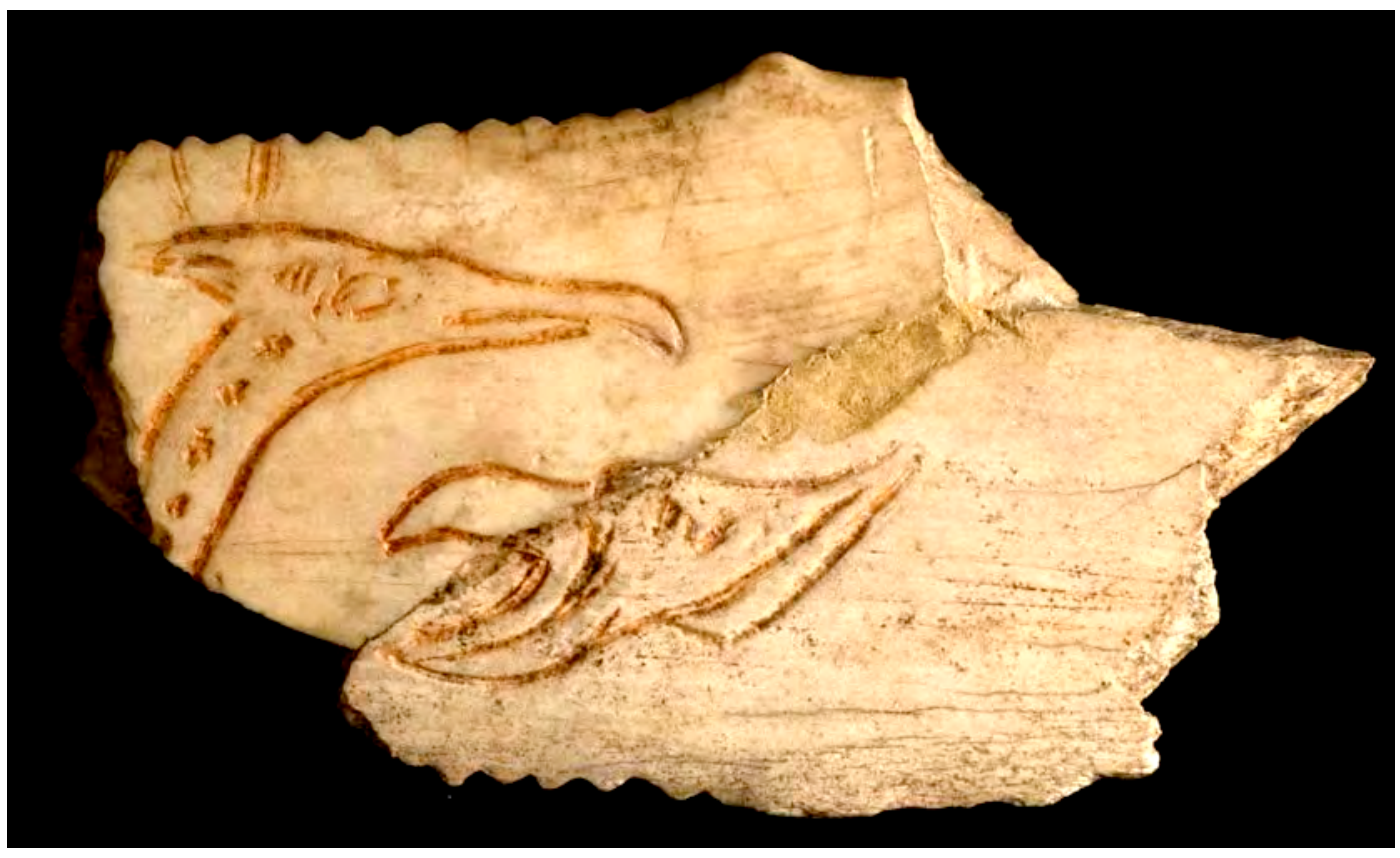

Figure 4 : Aurochs gravé sur lissoir, Rochereil (Musée national de Préhistoire). Cliché Émilie Lesvignes.

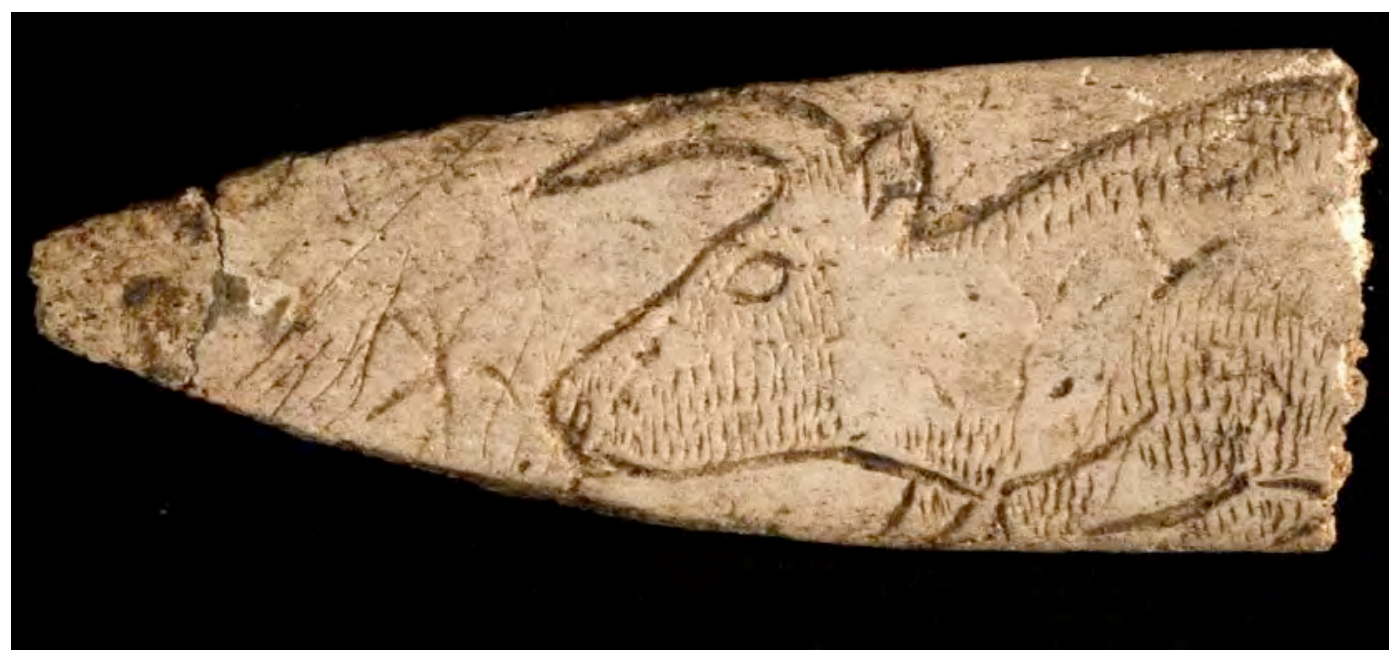


Figure 5: Renne accroupi gravé sur plancher stalagmitique (panneau C), La Mairie Teyjat. Cliché Jean-Christophe Domenech.

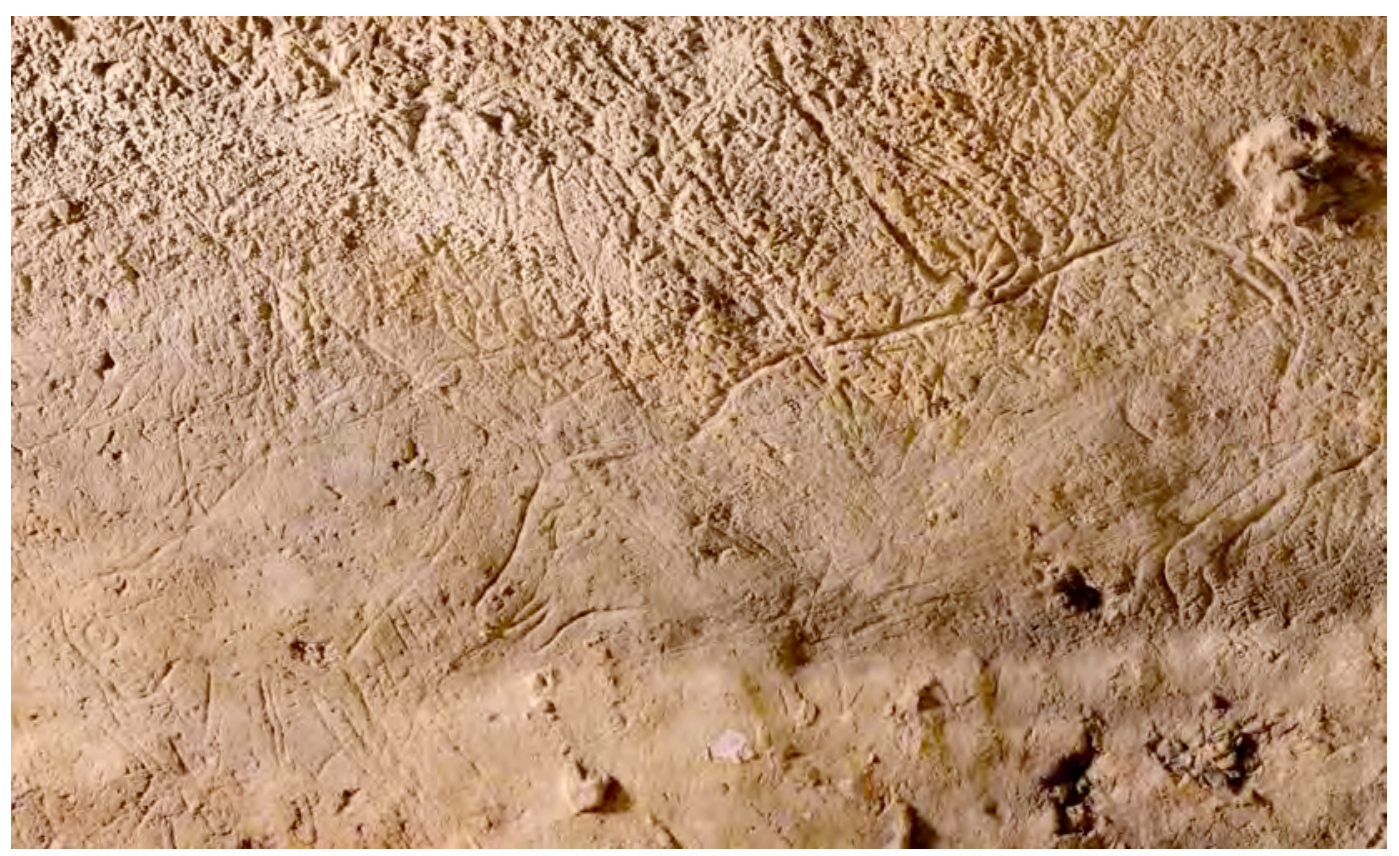

Figure 6 : Bisons se suivant gravés sur plancher stalagmitique (panneau D), La Mairie Teyjat. Relevé sélectif Patrick Paillet.

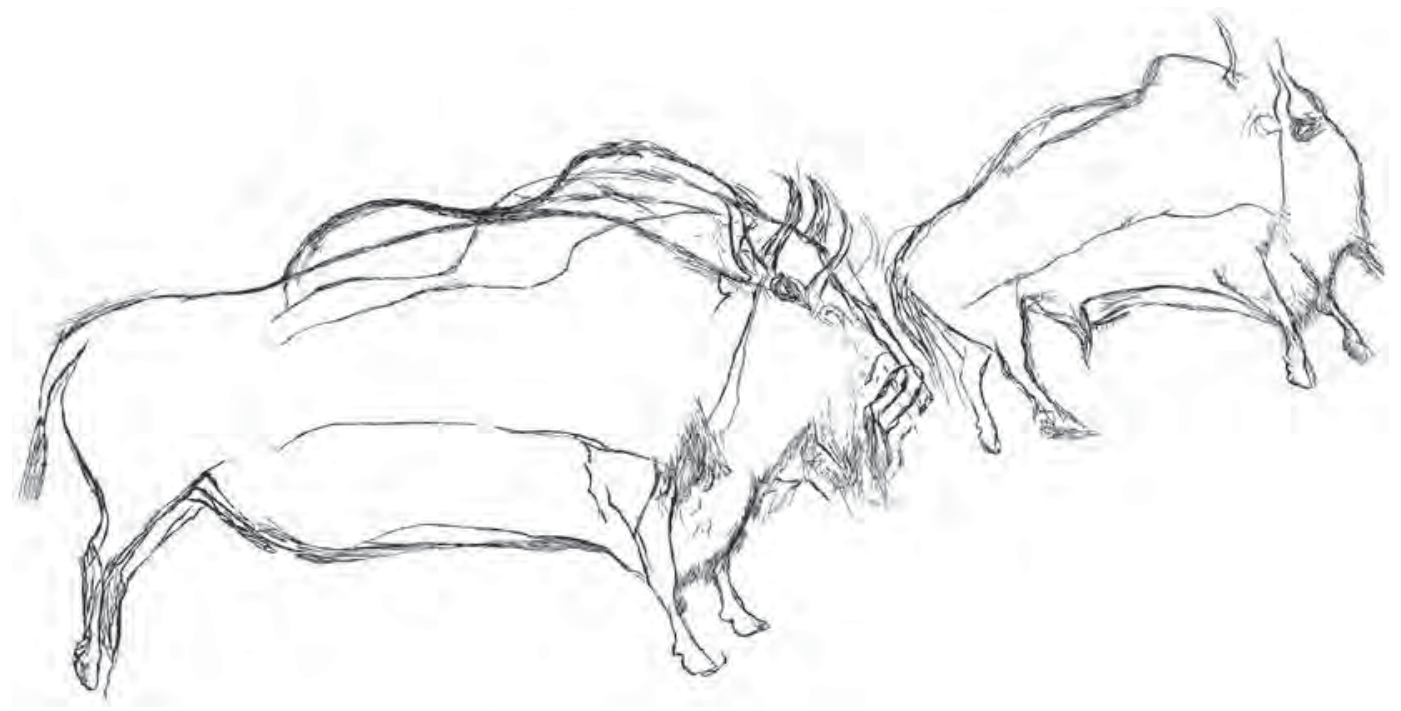


Figure 7 : Aurochs, chevaux, rennes, etc. gravés sur cascade stalagmitique (panneau F), La Mairie - Teyjat. Relevé Patrick Paillet.

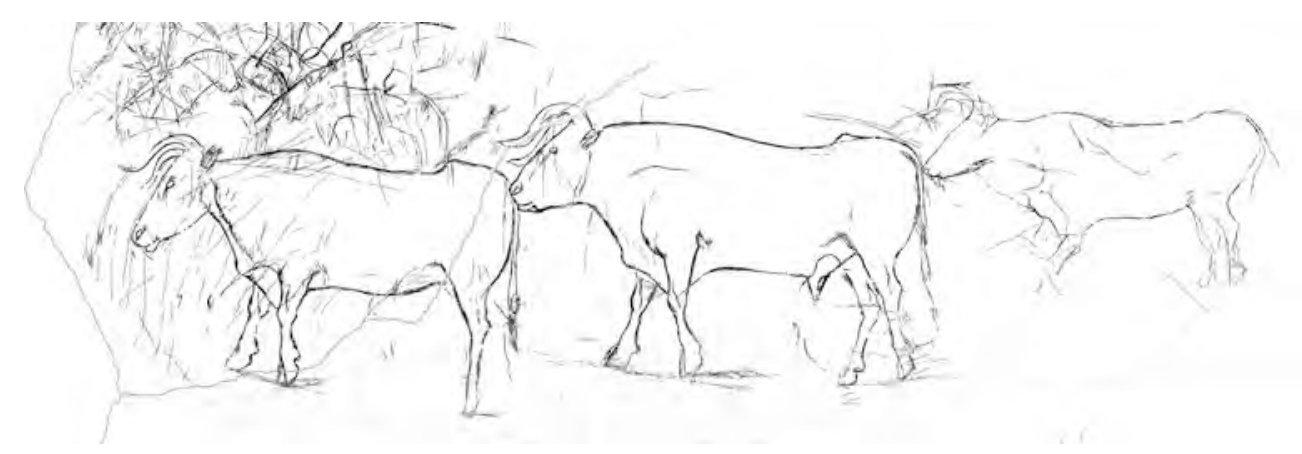

Figure 8: Fragment de scapula gravée d'arrière-trains d'animaux, La Mairie - Teyjat (Musée d'Archéologie nationale). Cliché Patrick Paillet.

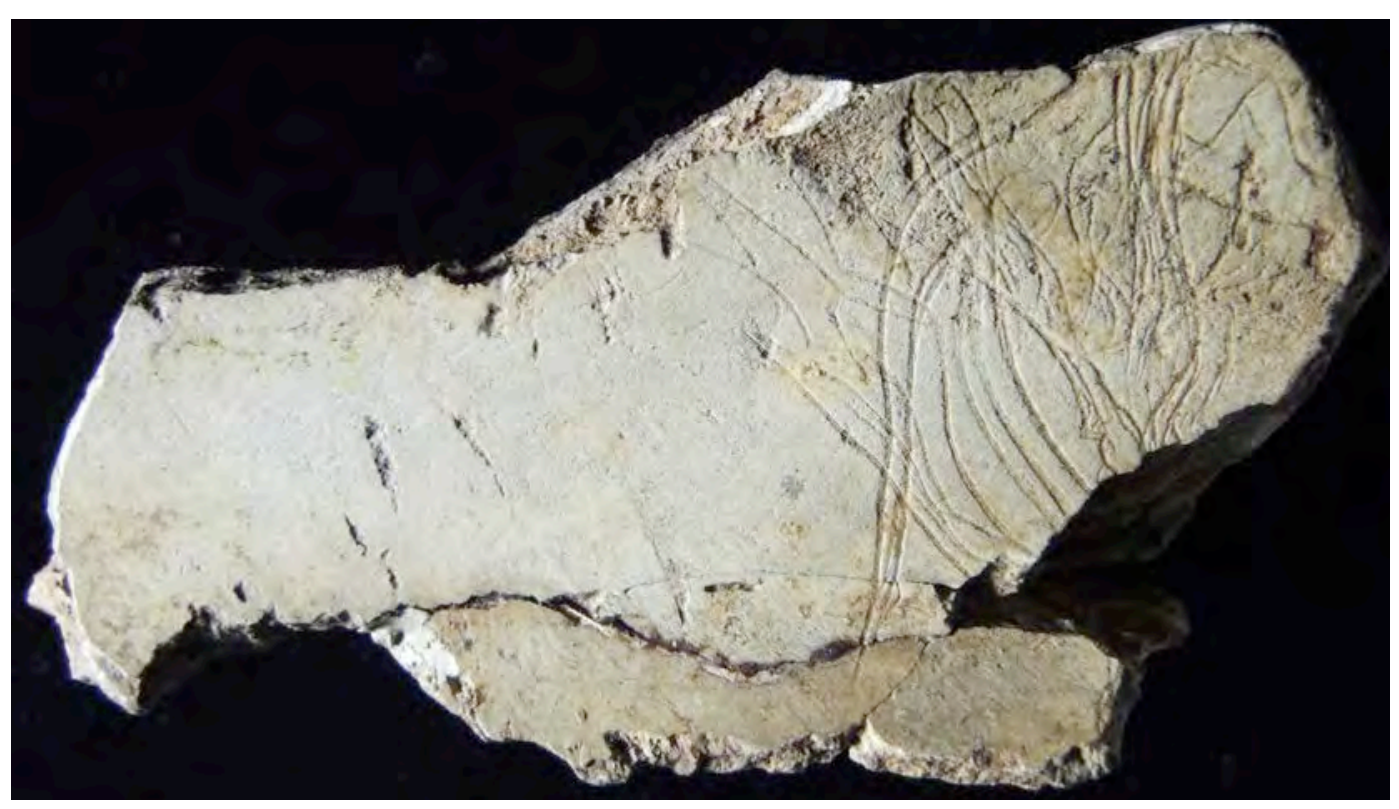


Figure 9: Fragment de scapula gravée d'un bison et d'un cheval, Rochereil (Musée national de Préhistoire). Cliché et relevé Patrick Paillet.
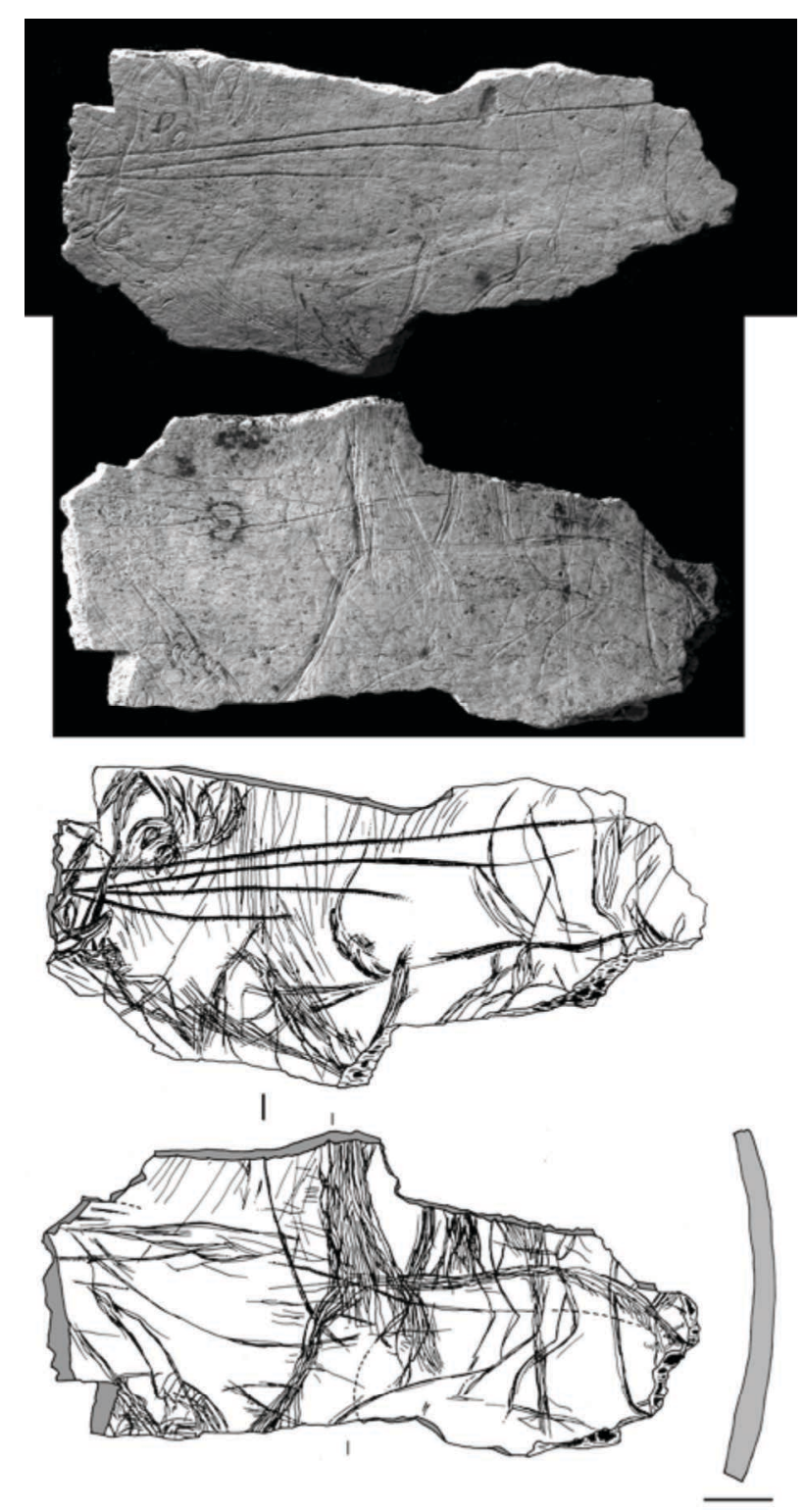
Figure 10: Rennes, bison et cerf gravés sur plancher stalagmitique (panneau B), La Mairie. Photo et relevé Patrick Paillet.

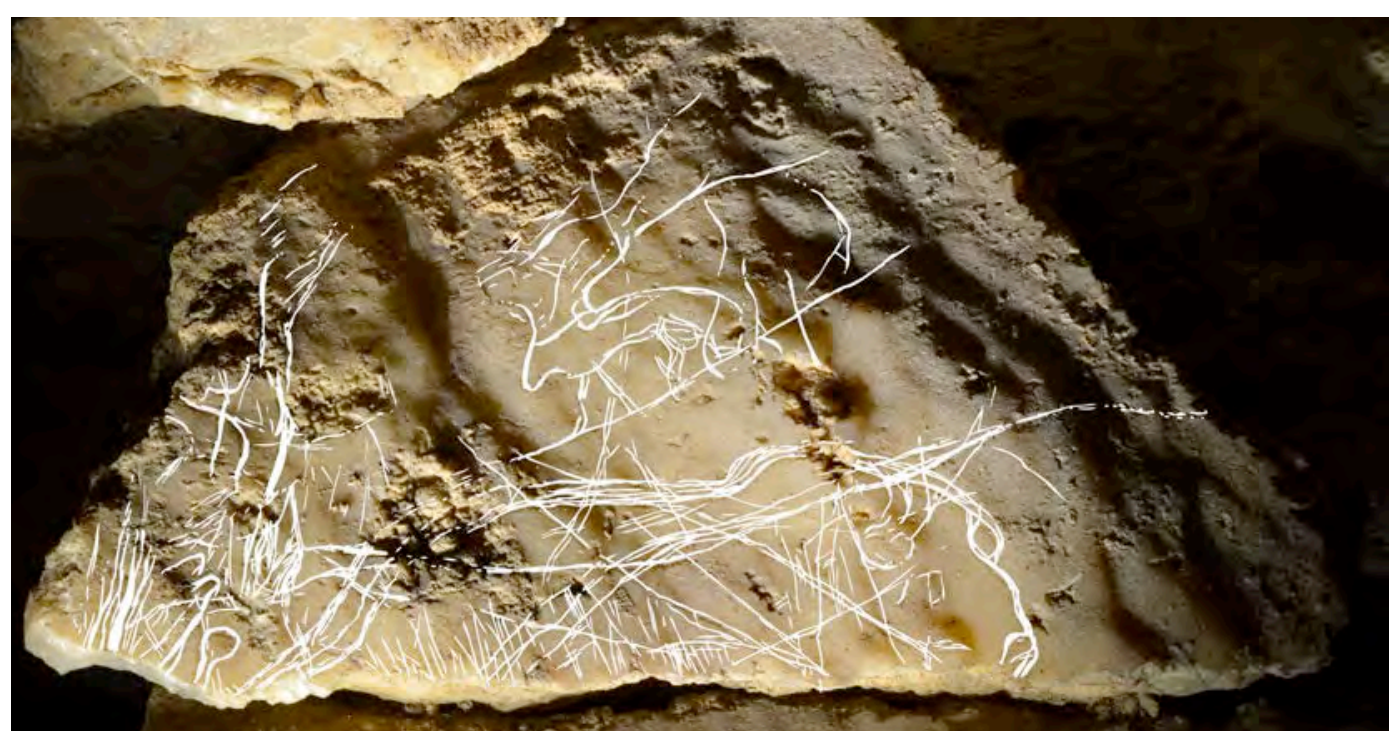

Figure 11: Chevaux macrocéphales gravés sur pièce biseautée, La Madeleine (British Museum). Dessin d'après Édouard Lartet et Henry Christy (1865-1875).

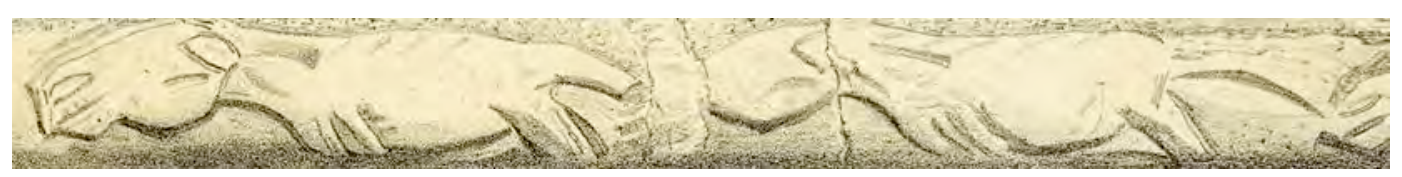


Figure 12 : Chevaux macrocéphales gravés sur pièce biseautée, Rochereil (Musée national de Préhistoire). Cliché Émilie Lesvignes et relevé Patrick Paillet.
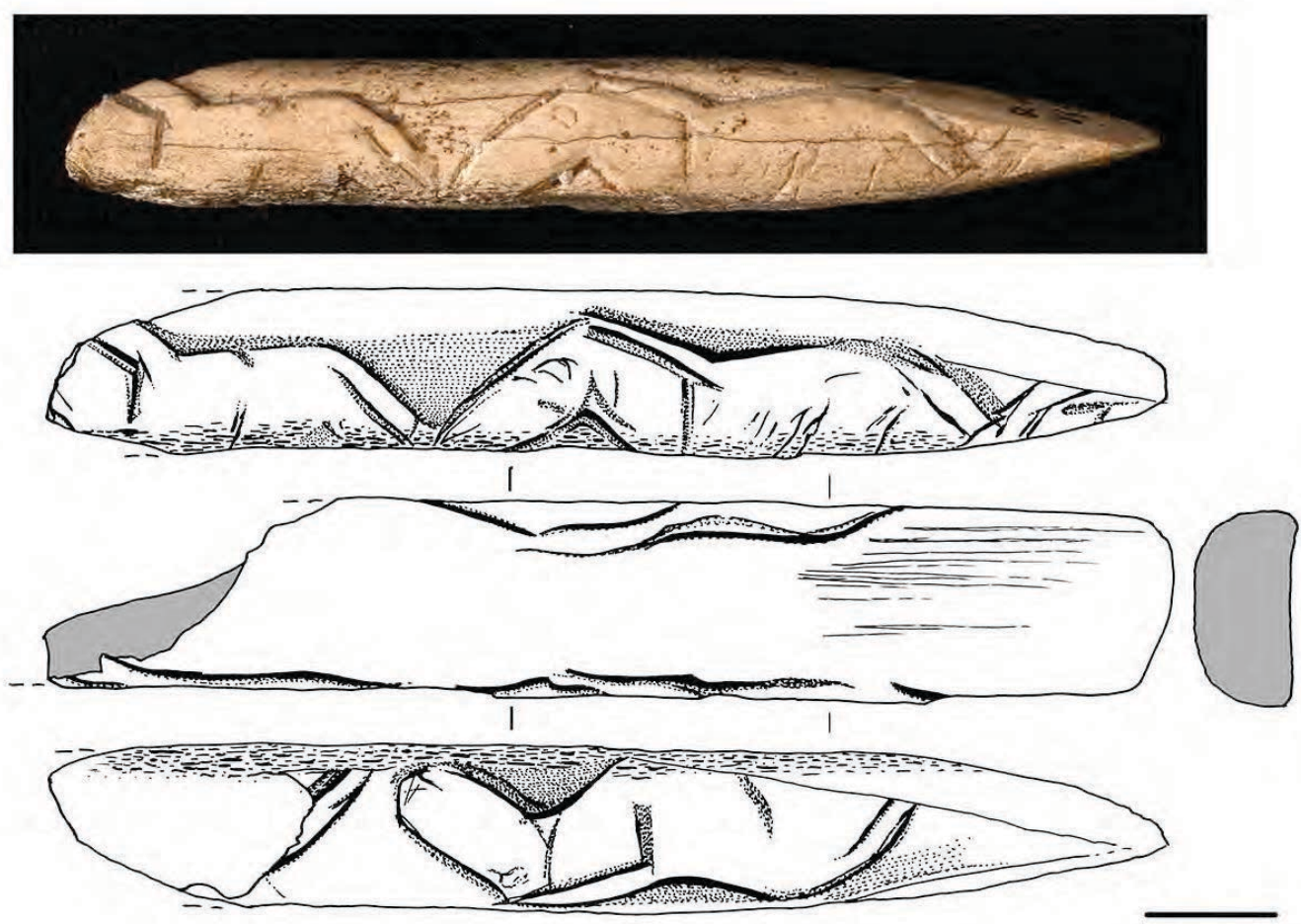

Figure 13: Chevaux macrocéphales gravés sur os d'oiseau, La Mairie -Teyjat (pièce disparue). Relevé Édouard Perrier du Carne (1889).

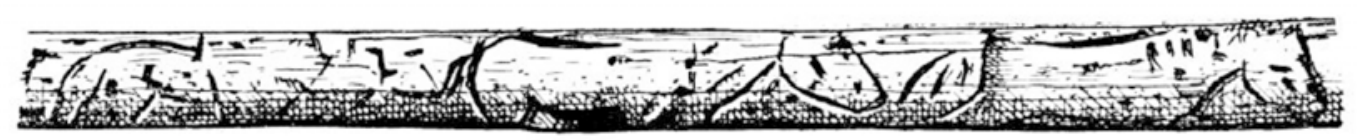


Figure 14 : Caprinés et cervidés représentés en vue conventionnelle de face. a : bouquetin et signes sur lame d'os découpée, Rochereil ; b : tête de bouquetin sur fût de harpon, Rochereil : c : tête de capriné (chamois ?) sur embase de harpon, Rochereil ; d : tête de bouquetin? sur fût de harpon, Rochereil (Musée national de Préhistoire); e : têtes de cervidés sur baguette demi-ronde, abri Mège et $\mathrm{f}$ : têtes de caprinés sur baguette demironde, La Mairie - Teyjat (Musée d'Archéologie nationale). Clichés : Émilie Lesvignes (a à d), Patrick Paillet (e et f).

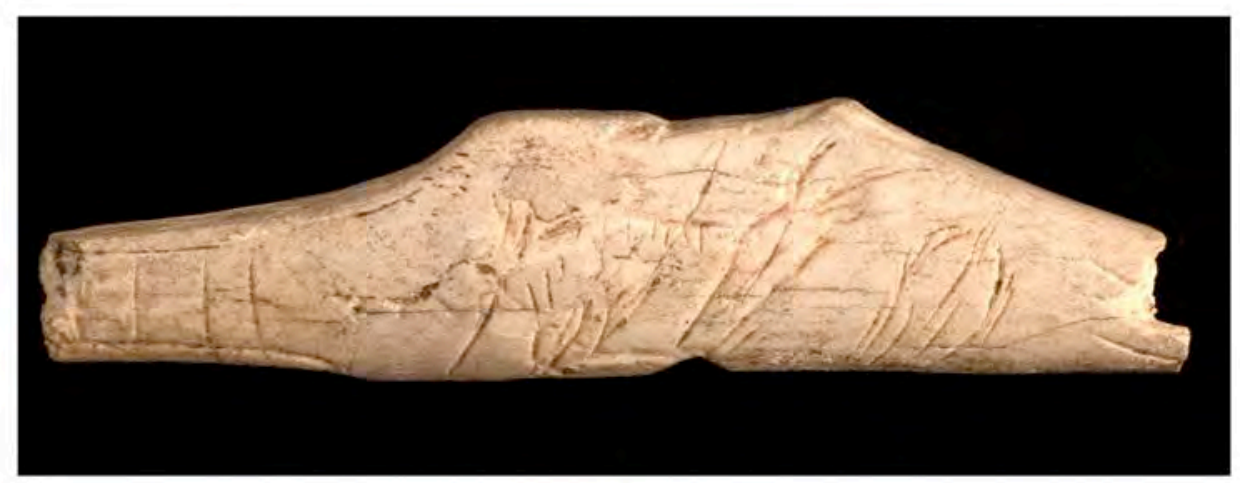

a

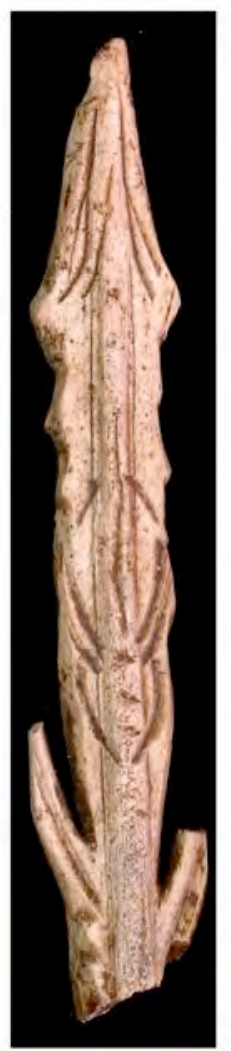

b

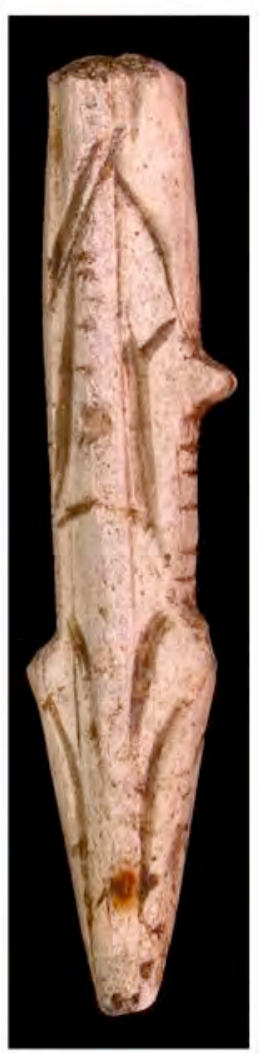

C

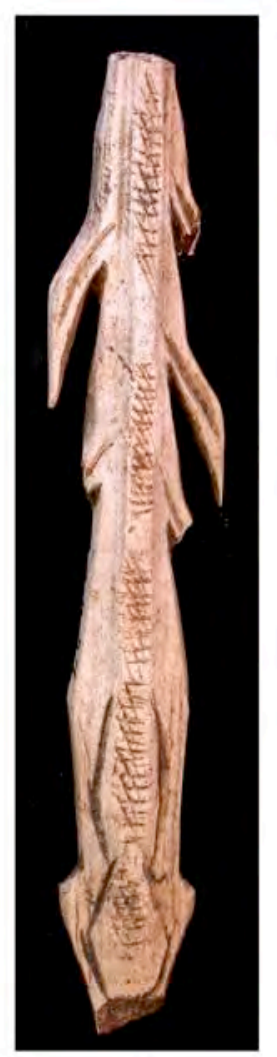

d

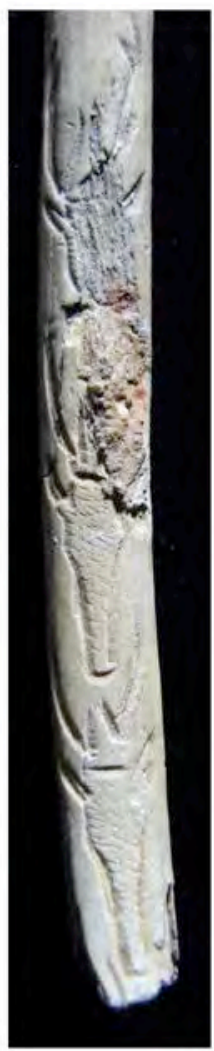

e

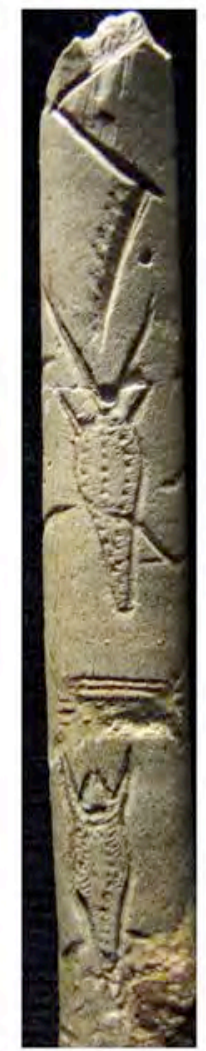

f 
Figure 15 : Bouquetin vu de face et schématisation de bouquetin (Llonin, Espagne). Cliché Patrick Paillet et relevé Javier Fortea Pérez.
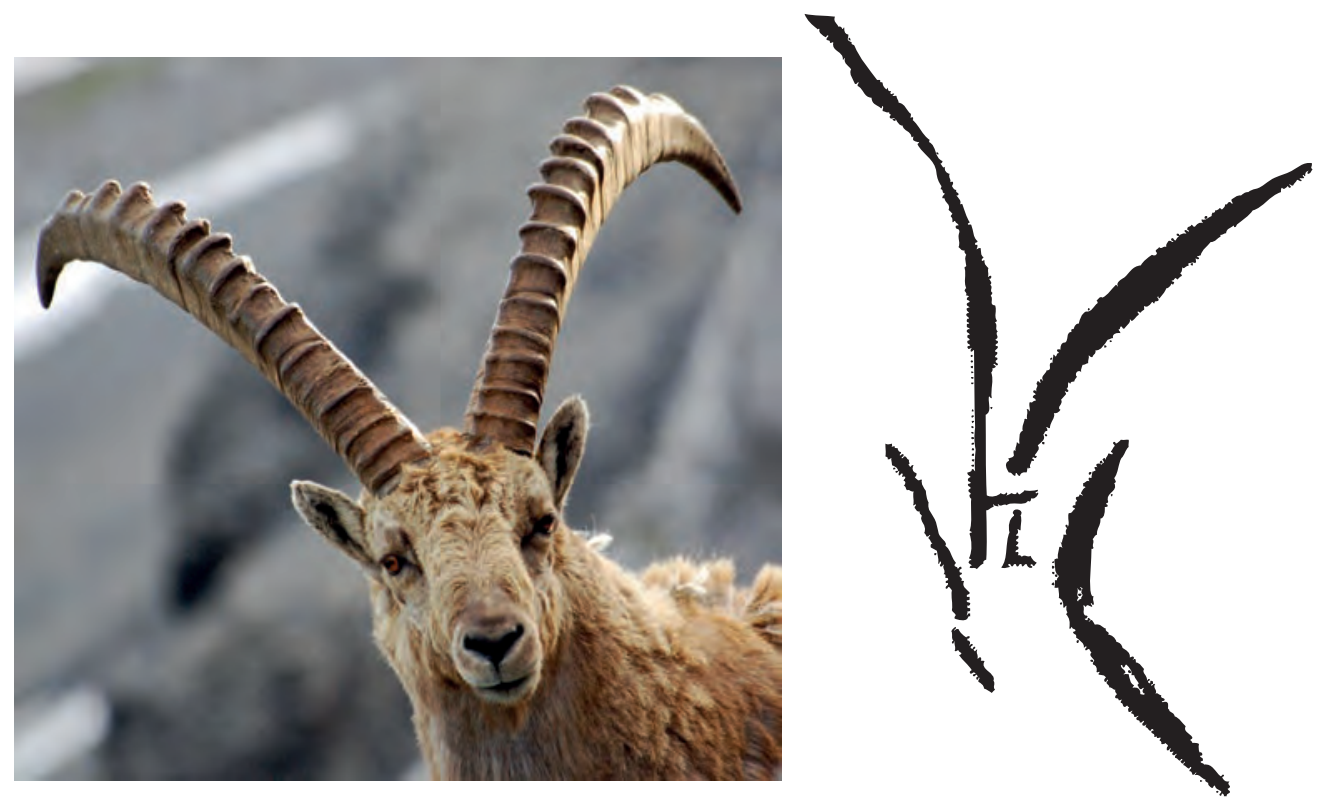

Figure 16 : Figures féminines schématiques gravées sur paroi, Fronsac (Dordogne). Cliché Patrick Paillet.

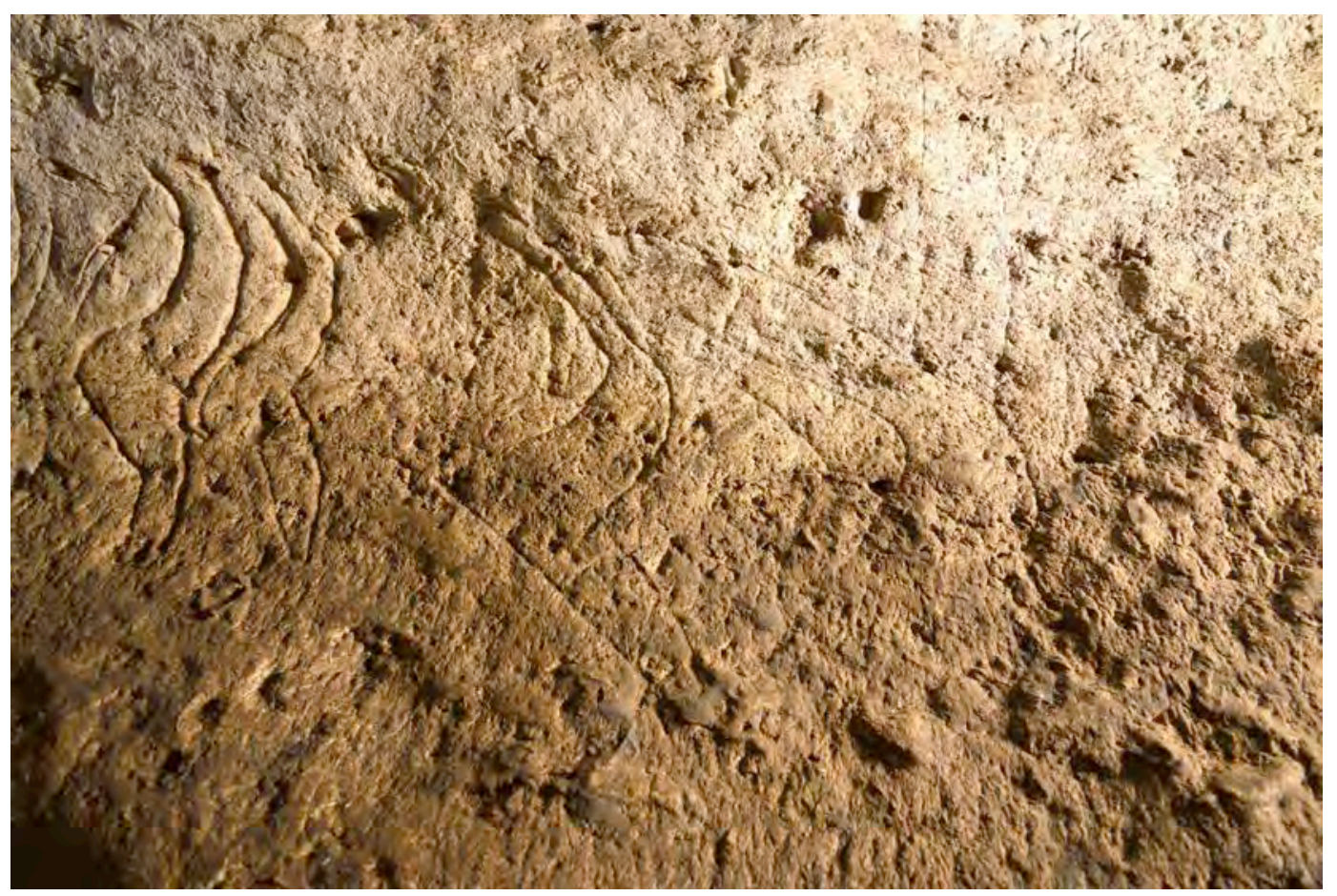


Figure 17: Représentations féminines schématiques de Rochereil (Musée national de Préhistoire). a : figure féminine de face gravée sur os d'oiseau; b : figure énigmatique (femme de face ?) gravée sur lame d'os découpée ; c : figure féminine schématique de face gravée sur lame d'os découpée; d : figure féminine de face et motif en «feuille » gravés sur diaphyse d'os long; e: figures féminines schématiques de profil gravées sur lame d'os; f : femme de face gravée sur diaphyse d'os long; g : figure féminine schématique gravée sur os d'oiseau. Clichés Émilie Lesvignes.

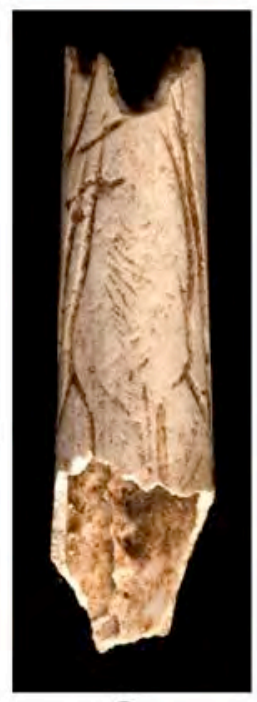

a

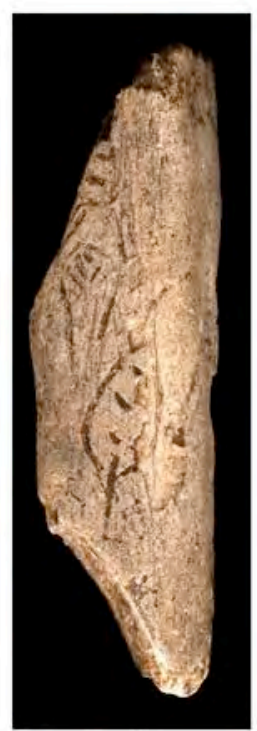

d

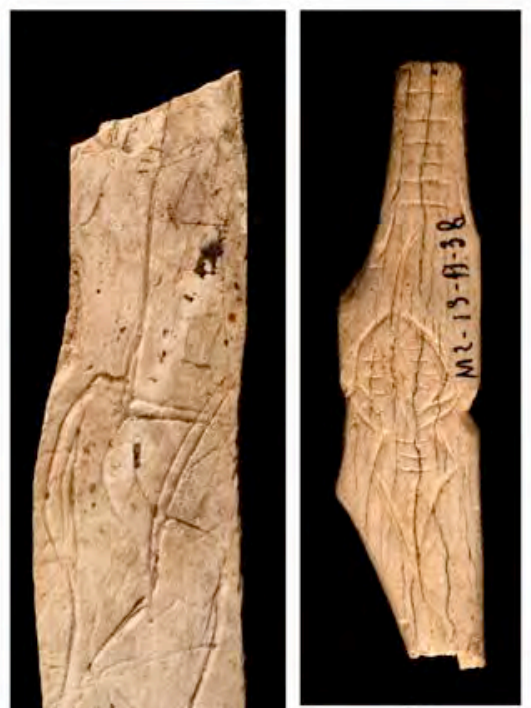

b

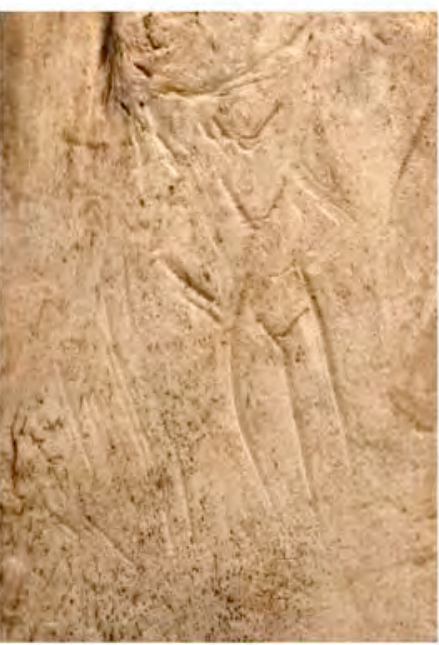

f

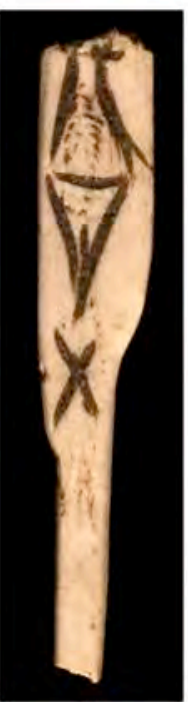

C

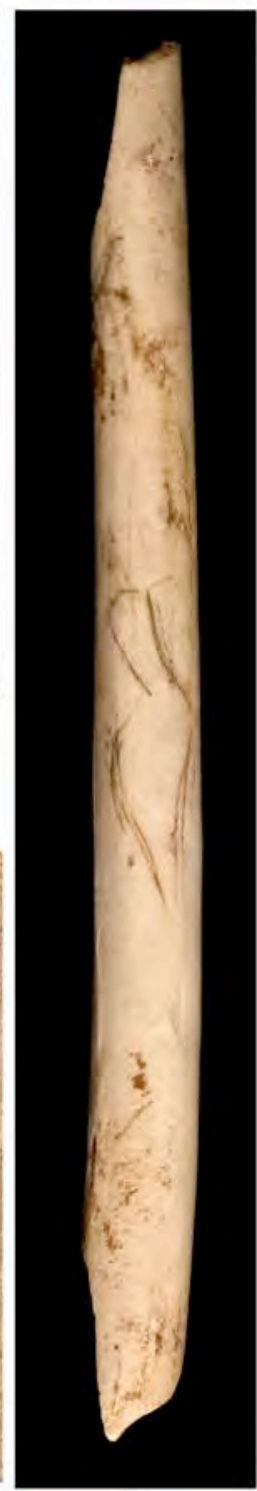

g 
Figure 18 : Motifs en forme de «feuille » et de « rave » de Rochereil (Musée national de Préhistoire). a : motif en « feuille » sur baguette de bois de renne; b : motif en « rave » sur pièce biseautée en bois de renne ; c : motif en « feuille » et figure féminine vue de face sur diaphyse d'os long; $\mathrm{d}$ : motifs en «feuille» et en «ogive» et oiseau sur outil intermédiaire ; e : motif en « rave » sur mandibule; f : motifs en «feuille » et « ogive » sur outil intermédiaire ; $g$ : motifs en « feuille » sur outil intermédiaire ; h : motifs en « rave » sur base de bois de renne. Relevés Patrick Paillet.
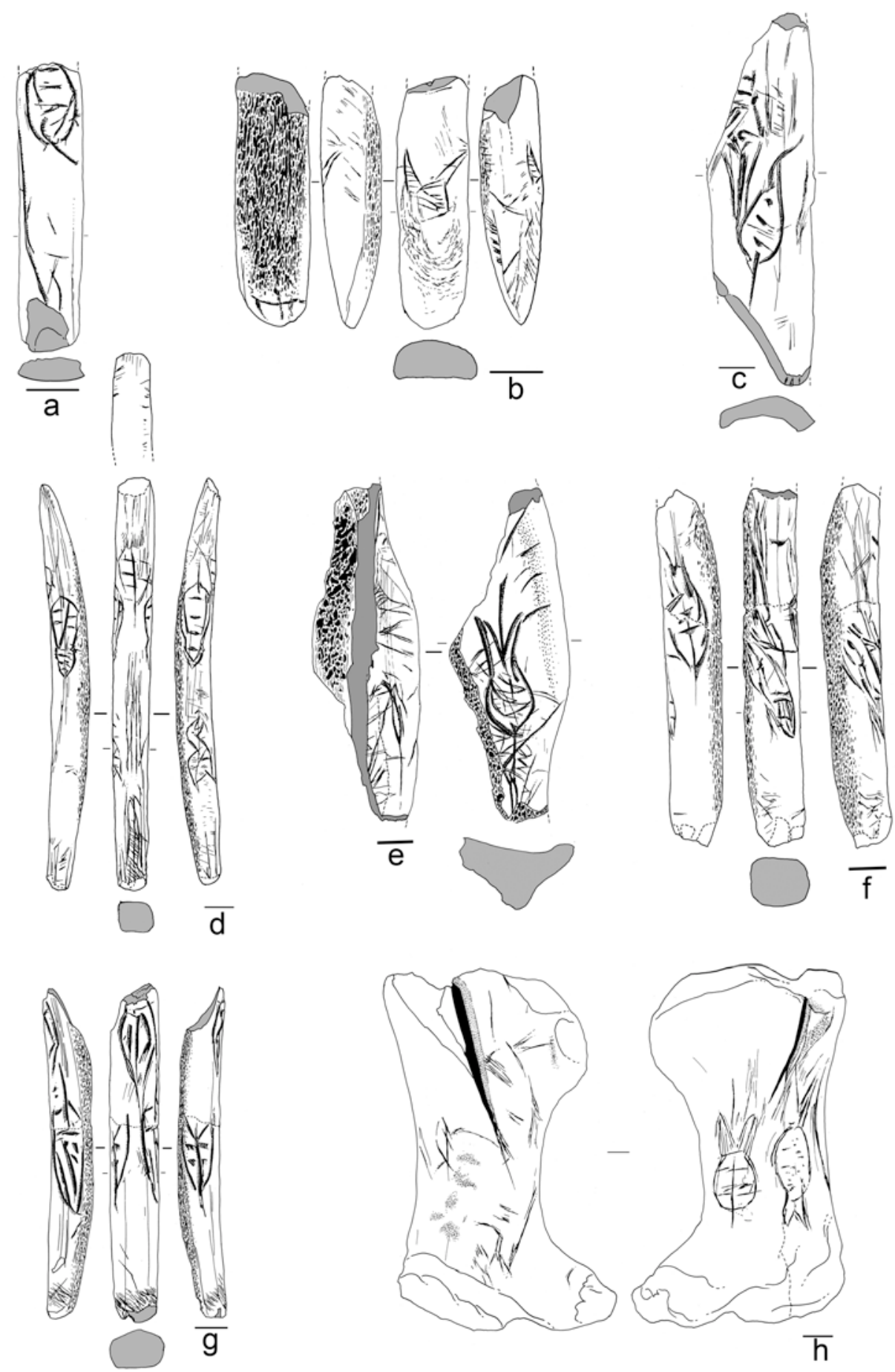
Figure 19: Bois de renne et côte gravés de motifs en «feuille » et de motifs énigmatiques (figures féminines vues de face?, segments d'animaux, etc.) (Musée d'Archéologie nationale). Relevés Henri Breuil (in Capitan et al. 1908).
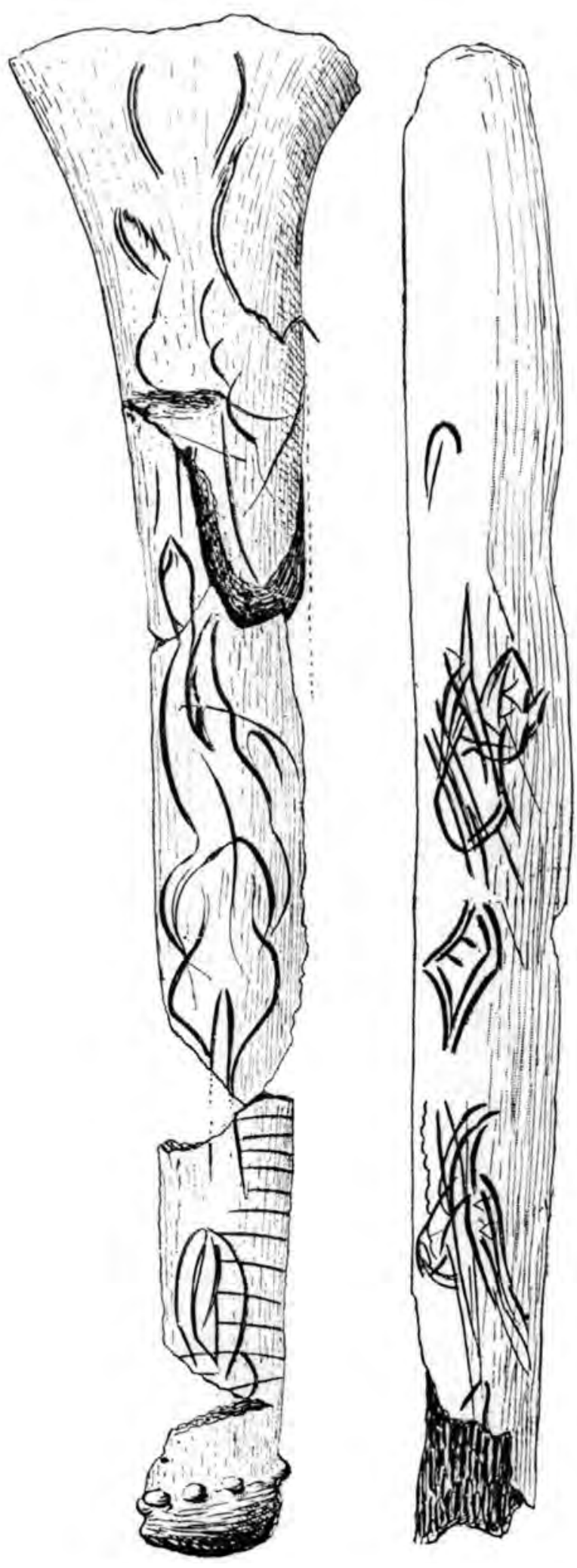


\title{
Les constructions symboliques : l'art préhistorique comme support de communication
}

\author{
Romain PIGEAUD, \\ Docteur en préhistoire, chercheur associé UMR 6566 « CREAAH » \\ du CNRS, Université de Rennes-1 \\ Florian BERROUET, \\ Docteur en préhistoire \\ Estelle BOUGARD, \\ Docteur en préhistoire, chercheur associé UMR 7194 du CNRS, \\ département de Préhistoire du Muséum national d'Histoire naturelle, \\ Institut de Paléontologie Humaine
}

Extrait de : Olivier BUCHSENSCHUTZ, Christian JEUNESSE, Claude MORDANT et Denis VIALOU (dir.),
Signes et communication dans les civilisations de la parole, Paris,
Édition électronique du CTHS (Actes des congrès des sociétés historiques et scientifiques), 2016.
Cet article a été validé par le comité de lecture des Éditions du CTHS dans le cadre de la publication
des actes du $139^{\mathrm{e}}$ Congrès national des sociétés historiques et scientifiques tenu à Nîmes en 2014.

\begin{abstract}
Résumé
En 1986, Denis Vialou définit les constructions symboliques comme des dispositifs dans lesquels « [...] les liens existant entre les thèmes et entre ceux-ci et leurs supports [...] sont généralement implicites et porteurs de significations ». Ce nouveau concept, qui fournit un cadre plus précis que les analyses d'André Leroi-Gourhan et d'Annette Laming-Emperaire, a engendré de nombreux travaux et analyses en grottes et abris ornés, comme sur les sites $\mathrm{d}^{\prime}$ art rupestre à l'air libre. Sur les parois, les associations d'animaux et de signes abstraits ainsi que les traces d'utilisations rituelles dépassent la simple juxtaposition de mythogrammes pour transmettre un message. La roche et le site rocheux ne sont plus de simples supports, mais interagissent avec ce message, pour le nuancer, le modifier, voire le médiatiser pour d'hypothétiques activités rituelles. À travers des exemples, nous présenterons quelques constructions symboliques exemplaires et discuterons sur le mode de transmission des messages ainsi laissés sur les parois.
\end{abstract}

Mots-clés: construction symbolique, mythogramme, mythe, caverne, naturalisme, éthologie

\begin{abstract}
In 1986, Denis Vialou defined symbolic constructions as ways for which “... existing links between themes and their medium [...] are generally implicit and hold much meaning". This new concept, which provides a more precise framework than the analyses of André Leroi-Gourhan and Annette Laming-Emperaire, has initiated studies and analyses of rock art in caves, rock shelters and open air sites. The association of animals with abstract symbols and the hints of ritual use exceed the simple juxtaposition of mythograms in order to transmit a message. The rock sites are not just simple media, but interact with this message in order to nuance, modify and transmit perhaps for hypothetical ritual activities. Using examples, we will present symbolic constructions and will discuss the transmission of these messages drawn on the rock face. (traduction Rebecca Peake)
\end{abstract}


En 1986, D. Vialou fonde le concept de «construction symbolique », dont il donne la définition suivante :

«Les dispositifs pariétaux sont des constructions symboliques dans la mesure où les liens existant entre les thèmes et entre ceux-ci et leurs supports et la grotte sont généralement implicites et porteurs de signification. » (Vialou 1986, p. 359)

Le terme de construction, plus opérant, fut finalement préféré à celui de charpente, qu'il avait précédemment employé (Vialou 1983, p. 94). Sous cet angle, le dispositif pariétal est bien plus qu'un décor: les manifestations graphiques s'articulent en bonne intelligence, car disposées selon des normes et créant des liaisons privilégiées qui aboutissent à une véritable construction. La durée de réalisation des représentations, leur juxtaposition et/ou superposition, les éventuelles reprises de tracés inscrivent le dispositif pariétal dans une dimension temporelle, tandis que la prise en compte de l'environnement - de l'échelle des microreliefs à celle du cadre géographique qui accueille la grotte ou le site de plein air, eux-mêmes supports influant sur l'origine et le devenir des figures - confère à ce dispositif une réalité spatiale. Cette approche spatio-temporelle, issue de la volonté d'étudier les sites de manière pluridisciplinaire et de ne pas occulter les questions inhérentes à leur conservation, est aujourd'hui indissociable des sciences préhistoriques. Le concept de construction symbolique est né en réaction à celui de mythogramme, proposé par A. Leroi-Gourhan (1964) et A. Laming-Emperaire (1962). Pour A. LeroiGourhan (1992, p. 275), un mythogramme est :

«Une figure ou un groupe de figures dont les éléments sont simplement juxtaposés, sans référence au temps ou à l'espace. »

Ce terme s'oppose au pictogramme :

«Figure ou groupe de figures coordonnées dans un réseau spatial et évoquant le temps et l'action par la figuration de mouvements significatifs »

et à l'idéogramme :

« Figure ou groupe de figures réduites à quelques traits ou à un tracé géométrique. »

Représentation symbolique d'un mythe, le mythogramme est un support qui se prête à l'interprétation.

D. Vialou suggère donc de s'inscrire dans un cadre plus précis, au sein du triangle «thème-support-grotte ». Le concept de construction symbolique permet également un plus large consensus parmi les différentes hypothèses avancées pour expliquer l'art pariétal : on s'accorde sur le fait que les animaux ne sont généralement pas représentés pour ce qu'ils sont, et que s'opère dans l'esprit et le geste des artistes un glissement vers le mythe, la caverne "participant» de cette architecture mentale. Toute la discussion portant alors sur ce qu'il faut entendre par « implicite ».

Notre propos ici est de partir de ce concept puis de le faire évoluer et de le préciser, en nous appuyant notamment sur quelques exemples pris dans l'aire franco-cantabrique ainsi que sur les opérations de relevé d'art pariétal dont l'un de nous (R. P.) a la charge : celles des grottes Margot (Thorigné-en-Charnie, Mayenne) et du Sorcier (Saint-Cirq, Dordogne). 


\section{Exemples de constructions symboliques}

D. Vialou fournit l'« holotype »d'une construction symbolique dans son article de 1982 : Niaux, une construction magdalénienne exemplaire. Dans le sanctuaire ariégeois, les animaux sont regroupés en panneaux dans le Salon noir, tandis que des signes sont figurés le long du cheminement depuis l'extérieur comme pour baliser le parcours (fig. 1). Ces signes sont répartis préférentiellement sur la paroi droite qui semble avoir servi de fil conducteur dans cette large galerie, mais on les trouve aussi sur la paroi gauche aux endroits plus étroits. Les liaisons entre les thèmes sont également explicitées, en termes mathématiques simples.

Une construction symbolique, c'est d'abord la combinaison de représentations, figures et signes, sur un mode aléatoire en apparence, mais qui obéit en réalité à une mise en scène graphique.

Le panneau orné de la Tête-du-Lion (Bidon, Ardèche, Combier 1984) montre ainsi un aurochs en profil gauche, que précède une encornure de bouquetin et surmonté de ponctuations alignées : il associe ainsi l'animal dans son entier, celui simplement suggéré par un caractère anatomique remarquable, et enfin les signes (fig. 2). À Mayenne-Sciences (Thorigné-en-Charnie, Mayenne), le panneau principal offre, dans une composition triangulaire savante, un cheval affronté à un mammouth, associé à trois autres chevaux et un signe triangulaire ovalisé (Pigeaud 2003, 2004) (fig. 3). Le célèbre panneau des deux «Chevaux ponctués » du Pech-Merle (Cabrerets, Lot, Lorblanchet 2010, p. 105 et sq.) est riche de mains négatives, de pochoirs de pouces, de nombreuses ponctuations et d'une représentation de poisson dessiné en rouge. La position tête-bêche de ces chevaux se retrouve notamment dans la galerie voisine du Combel (Cabrerets, Lot, Lorblanchet 2010, p. 179 et sq.) et à Mayenne-Sciences, permettant d'émettre l'hypothèse de liens culturels avec ce dernier site septentrional. Enfin, terminons cette revue de détail avec la grotte du Sorcier à Saint-Cirq (Dordogne) déjà citée, où la reprise récente de l'étude des gravures a permis de mettre en évidence une construction graphique élaborée autour de l'anthropomorphe ithyphallique, le "Sorcier », qu'environnent un remarquable profil de bison, un cheval, un petit félin et quelques silhouettes fantomatiques, le tout disposé en bordure d'une grande faille qui court au plafond (Pigeaud et al. 2012) (fig. 4).

\section{Interprétations et évolution du concept de construction symbolique}

Différents préhistoriens ont interprété le concept de construction symbolique et l'ont fait évoluer.

\section{Interprétation « canonique »}

Denis Tauxe est celui qui est resté le plus proche de $1^{\prime}$ «holotype ». Son travail sur la grotte de Lascaux en porte témoignage (2007). Cette cavité renferme quelque 600 figures animales identifiées et plus de 400 signes, certains thèmes comme les lignes ponctuées, par exemple, étant propres à des secteurs ou dispositifs; il est donc raisonnable de les interpréter par des orientations symboliques différentes (Tauxe 2007, p. 264). Par exemple, le cheval conserve une place éminente (c'est l'animal le plus représenté dans l'art paléolithique), l'aurochs domine le bison tandis que le cerf est prééminent sur le bouquetin, ou encore l'association cheval-aurochs se rencontre dans tous les secteurs de la grotte. En outre, la base du système peint repose principalement sur l'alternance chevaux/bovins et bovins noirs/bovins rouges, à laquelle s'ajoute l'intervention ponctuelle du cerf ou du bouquetin (fig. 5). Le cerf et le cheval s'associent de part et d'autre d'une ligne de points. Par ces observations qui permettent d'entrevoir comme l'ébauche d'une "grammaire » graphique, les principales espèces animales apparaissent comme des identités culturelles; certains signes caractéristiques parfois complexes 
(branchiformes, signes en damier...), venant encadrer et accompagner le dispositif, deviennent alors les éléments moteurs de croyances distinctes. À ce sujet, le rapprochement avec la grotte proche de Gabillou (Sourzac, Dordogne, Gaussen 1984, p. 231) a souvent été mis en avant, quoique nuancé depuis (Petrognani et Sauvet 2012).

\section{Interprétation naturaliste}

À Lascaux, Norbert Aujoulat, qui en a longuement examiné et photographié les parois, a repéré des indices de saisonnalité dans la manière de figurer certaines espèces (Aujoulat 2004, p. 187 et sq.). La robe des chevaux évoque la fin de l'hiver ou le début du printemps ; les aurochs arborent leur livrée estivale ; enfin, les cerfs sont représentés avec leurs attributs d'automne. Il s'agit là, pour ces espèces, de la période correspondant aux prémices de l'accouplement, au cours de laquelle elles connaissent un regain d'activité. Mais il est particulièrement intéressant de remarquer que ces animaux ont été figurés sur les parois de la grotte dans cet ordre-là, comme l'a montré l'étude des superpositions et de l'utilisation de l'espace graphique. À l'échelonnement de ces événements au cours d'une année se superpose une chronologie de réalisation. Cycles, renaissance, mythe de l'éternel retour ?... Laissons là les pistes d'interprétation pour nous contenter de saluer, à l'instar de N. Aujoulat, cette « fantastique ode à la vie » (ibid., p. 194).

\section{Interprétation éthologique}

Pour plusieurs sites fameux de l'art pariétal franco-cantabrique, dont les images offrent des styles parfois très différents, le soin porté à certains détails anatomiques, la cohabitation d'espèces prédatrices et d'espèces-proies ou l'agencement des figures dans l'espace souterrain autorisent une lecture éthologique, c'est-à-dire en lien avec les comportements animaux. Dans la Salle du Fond de la grotte Chauvet-Pont-d'Arc (VallonPont-d'Arc, Ardèche, Clottes dir. 2001, p. 128, fig. 125), le panneau le plus remarquable et le plus densément peuplé de la grotte paraît relater la chasse d'un troupeau de bisons par une meute de lions; la scène est saisissante de réalisme et rendue particulièrement vivante grâce à l'emploi de la perspective et de l'estompe (Azéma 2010, p. 134 ; 2011, p. 37) (fig. 6). Ce comportement de prédation est aussi exprimé par les lions gravés de Roucadour (Thémines, Lot); cette fois-ci aux prises avec un mammouth très stylisé qui se surimpose aux félins (Lorblanchet 2009). La chasse aux mammouths est aussi au cœur du plafond de Baume Latrone (Sainte-Anastasie, Gard), ou un grand félin central extrêmement stylisé est entouré d'une suite de mammouths de traitement graphique similaire (Azéma 2011, p. 92-93) (fig. 7). À Chauvet-Pont-d'Arc, une famille d'ours des cavernes, reconnaissables à leur «stop » frontal, a été dessinée en rouge : une femelle à l'épaule marquée par un puissant relief de la paroi, accompagnée de son petit, précède un mâle (Clottes dir. 2001, p. 72, fig. 62 ; Azéma 2010, p. 431, tableau 5B). Dans l'Abside de Lascaux, les célèbres "bisons adossés", dont une plage de pigment rouge sur le pelage de celui de gauche figure la bourre, s'apprêtent à combattre (Aujoulat, op. cit., p. 178-179, fig. 128).

\section{Retour à la grotte}

L'art paléolithique expose bien un "message codé »; cependant, la définition précise, mais néanmoins générale, proposée par $\mathrm{D}$. Vialou ne saurait se contenter d'une unique et immuable mise en situation (Vialou 1989). Chaque grotte est originale, du fait de sa localisation dans un cadre géographique, par sa topographie et ses possibilités de cheminement, par l'attrait qu'elle exercera - ou pas - sur les hommes et femmes préhistoriques susceptibles d'y œuvrer à l'exécution d'un décor... Au sein du milieu souterrain, les formes de relief sont déterminantes dans la manière d'appréhender l'espace; volontairement négligées dans l'exposé précédent des différentes interprétations, elles n'en restent pas moins le substrat de départ, le support qu'investiront les artistes (Lejeune 1985 ; Sauvet, Tosello 1998). Citons deux exemples, 
parmi des centaines : à la grotte Margot (Thorigné-en-Charnie, Mayenne), une tête de rhinocéros laineux a été gravée à partir d'une fissure (Pigeaud, Hinguant et al. 2010) (fig. 8). Dans la grotte du Sorcier à Saint-Cirq (Dordogne), le panneau du «Sorcier» s'articule à la voûte par tout un entrelacs de gravures de facture différente au niveau d'une diaclase, le long d'un alignement de petites coupoles (Pigeaud et al. 2012, p. 242, fig. 13).

L'alternance d'espaces larges et d'autres restreints est une donnée physique incontestable dans les grottes. A. Leroi-Gourhan l'avait intégrée dans sa réflexion sur l'organisation symbolique des cavités ; l'espace long serait pour lui masculin et l'espace large, féminin (Leroi-Gourhan 1970, p. 238-241, fig. 145). Dans la grotte Margot, cette alternance rythme la circulation dans la cavité, et le décor semble s'y conformer: par exemple, des représentations d'oiseaux sont placées à chaque espace de transition, ou bien un espace exigu semble appeler la réalisation d'un grand nombre de gravures (Pigeaud, Hinguant et al. 2012). On remarque en particulier une certaine appétence pour les espaces étroits, et la volonté de s'y engager jusqu'à épuisement des possibilités, comme cela est perceptible par exemple au Combel (Cabrerets, Lot) ou au Pergouset (Saint-Géry, Lot) (Lorblanchet $2001 ; 2010$, p. 190).

Le potentiel d'ornementation d'une cavité diffère donc d'un site à l'autre, et il échappe aux préhistoriens dans la mesure où il n'est pas seulement dicté par quelque logique topographique (certaines plages de roche immaculée seront parfois laissées vierges au bénéfice de niches ou diverticules malcommodes d'accès), mais obéit à des impératifs d'ordre sensible ou symbolique qui leur restent inaccessibles (Pigeaud 2013). Il existe un « programme iconographique » qui reste à expliciter (Gonzalez 1998 ; 2001, p. 9).

\section{Retour à la définition}

Tandis que les mythogrammes constituent des éléments juxtaposés, D. Vialou a ajouté à la réflexion l'idée d'espace, d'une véritable architecture topographique correspondant à une appropriation mentale du milieu souterrain. Ce volet implicite, qu'il est difficile d'appréhender, de chiffrer, de systématiser, paraît jouer un rôle majeur dans les constructions symboliques. Dans la grotte du Sorcier, par exemple, au fur et à mesure que la lecture s'affine et ne considère plus les entités graphiques isolément au bénéfice du maillage général, un nombre toujours croissant de silhouettes dites fantomatiques, d'anthropomorphes juste ébauchés ou plus détaillés sont découverts. Les figures majeures, identifiées par les lectures précédentes et qui empruntent au registre "classique » de l'art pariétal (chevaux, bisons et, plus rares, anthropomorphe et félin) (Delluc et al. 1987, fig. 33), paraissent reliées, ponctuées par ces silhouettes ainsi que par de petites figures féminines schématiques (fig. 9). De même, on retrouve au Pergouset (Saint-Géry, Lot) des silhouettes fantomatiques, certaines monstrueuses, associées à des représentations vulvaires (Lorblanchet 2001) (fig. 10). Ces vulves et personnages, agencés le long d'un couloir, qui accompagnent la progression dans l'espace de la grotte, ne sont pas sans évoquer une sorte de panorama mythifié des origines, une histoire qui aurait été contée là, au Magdalénien. Des vulves gravées utilisant le relief naturel sont connues également dans le réseau Guy Martin (Lussac-les-Châteaux, Vienne ; Airvaux 1998, p. 508, fig. 6) ou à Margot (Pigeaud, Hinguant et al. 2012, p. 93, fig. 14 ; p. 94, fig. 16). Dans cette dernière cavité, une représentation sexuelle féminine occupe une position centrale tout au cœur du sanctuaire (galerie du Chêne pétrifié), au niveau du secteur le plus densément orné de la grotte, à proximité d'une large fissure et d'un épais pilier stalagmitique (fig. 11). 
Le grand panneau de la Salle du Fond dans la grotte Chauvet-Pont-d'Arc, précédemment évoqué, incontestable apogée du plus vieux sanctuaire orné de l'humanité, touche à la perfection à plus d'un titre: procédés graphiques mis en œuvre, expressivité des nombreux animaux figurés, caractère animé de cette probable scène de chasse où chaque protagoniste fait corps avec la paroi, en une remarquable utilisation des formes de relief, jusqu'à ce cheval central solitaire niché au fond de son alcôve. Au-delà de l'aspect naturaliste, le caractère puissamment symbolique de cette construction graphique s'impose. Chaque unité de cette folle sarabande pourrait constituer un mythogramme.

A. Leroi-Gourhan avait ainsi établi la construction symbolique comme un assemblage de ces mythogrammes; D. Vialou en a fait une réunion de symboles, avec un ancrage topographique spécifique à l'espace de la grotte et à la faveur des reliefs pariétaux, et un ancrage temporel, plus « universel », de par la perduration de cet art et de son évolution (à laquelle sont rattachées les questions de conservation), mais aussi en raison de la possibilité d'y lire des mythes universels (Pigeaud 2005).

Le concept de la construction symbolique tourne autour du triangle «thème-supportgrotte », appelé à se déformer suivant l'espace de la cavité et la durée de son exploration. Il s'instaure alors un dialogue à trois interlocuteurs : l'artiste préhistorique et ses images, le préhistorien, la grotte et sa paroi. L'artiste interroge la paroi pour l'utilisation des reliefs, le choix des panneaux à orner, paramètres possiblement dictés par des impératifs d'ordre symbolique. Le préhistorien questionne l'artiste pour tenter d'interpréter cet art ; il examine également la paroi pour comprendre la prise en compte des reliefs. Au final, élaborer une construction symbolique, c'est passer d'animaux réels, issus d'une faune peuplant l'environnement physique des hommes de la Préhistoire, à un bestiaire (c'est-àdire une sélection d'animaux à valeur de mythes, figurés selon divers degrés de stylisation), projeté dans un paysage mental, celui voulu par l'(les) artiste(s) face à la paroi. Davantage qu'un exercice abstrait, identifier les constructions symboliques permet de retrouver toute la mécanique intellectuelle déployée par les hommes préhistoriques, et par-delà, les identifiants de la société qui l'a employée (Petrognani 2013). Ce n'est pas de l'histoire de l'art, c'est de l'archéologie. 


\section{Bibliographie}

AIRVAUX J. 1998. Découverte d'une grotte ornée, le Réseau Guy Martin à Lussac-LèsChâteaux, Vienne, et application d'une méthodologie structurale pour l'étude de l'art préhistorique, L'Anthropologie, vol. 102, n 4, p. 495-521.

Aujoulat N. 2004. Lascaux. Le geste, l'espace et le temps, Paris, Le Seuil, coll. "Arts rupestres $», 274 \mathrm{p}$.

AZÉMA M. 2010. L'art des cavernes en action, tome 2 : les animaux figurés, Paris, Errance, coll. « Hespérides », $472 \mathrm{p}$.

AZÉMA M. 2011. La Préhistoire du cinéma, Paris, Errance, 304 p.

Clottes J. (dir.) 2001. La Grotte Chauvet. L'Art des Origines, Paris, Le Seuil, 224 p.

Clottes J. 2010. Les Cavernes de Niaux. Art préhistorique en Ariège, Paris, Errance, 232 p. (1 $1^{\text {ere }}$ édition 1995).

COMBIER J. 1984. Grotte de la Tête-du-Lion, in Collectif, L'art des cavernes. Atlas des grottes ornées paléolithiques françaises, Paris, coéd. Ministère de la Culture / Imprimerie nationale, p. 595-599.

Delluc B., Delluc G., Guichard F. 1987. La grotte ornée de Saint-Cirq (Dordogne), Bulletin de la Société préhistorique française, t. 84, $\mathrm{n}^{\circ} 10-12$, Hommage de la Société préhistorique française à André Leroi-Gourhan, p. 364-393.

GAUSSEN J. 1984. Grotte de Gabillou, in Collectif, L'art des cavernes. Atlas des grottes ornées paléolithiques françaises, Paris, coéd. Ministère de la Culture / Imprimerie nationale, p. 225-231.

GONZALEZ R. 1998. Organisation spatiale et programme décoratif, une nouvelle proposition pour l'étude de l'art rupestre paléolithique européen, International Newsletter on Rock Art, 20, p. 24-26.

GONZALEZ R. 2001. Art et espace dans les grottes paléolithiques cantabriques, Grenoble, éd. Jérôme Millon, coll. « L’Homme des Origines », 464 p. (traduction M.-C. GROENEN).

LAMING-EMPERAIRE A. 1962. La signification de l'art rupestre paléolithique, Paris, éd. Picard, $424 \mathrm{p}$.

LEJEUNE M. 1985. La paroi des grottes, premier «mur» support artistique et document archéologique, Art \& Fact, ${ }^{\circ}$ 4, Liège, p. 15-24.

LeROI-GOURHAN A. 1970. Le geste et la parole. II. La mémoire et les rythmes, Paris, éd. Albin Michel, 288 p. (1 ${ }^{\text {ère }}$ édition 1965).

LeROI-GOURHAN A. 1992. L'art pariétal, langage de la Préhistoire, Grenoble, éd. Jérôme Millon, coll. «L'Homme des Origines », $420 \mathrm{p}$.

LORBLANCHET M. 2001. La grotte ornée de Pergouset (Saint-Géry, Lot): un sanctuaire secret paléolithique, Paris, éd. Maison des Sciences de l'Homme, Documents d'Archéologie française, $n^{\circ} 85,188 \mathrm{p}$.

LORBLANCHET M. 2009. Le combat des lions et des mammouths: un récit des temps glaciaires, Préhistoire du Sud-Ouest, n 17, fasc. 2, p 235-242.

LoRblanchet M. 2010. Art pariétal. Grottes ornées du Quercy, Rodez-Arles, éd. du Rouergue, $445 \mathrm{p}$. 
Petrognani S. 2013. De Chauvet à Lascaux, l'art des cavernes, reflet de sociétés préhistoriques en mutation, Arles, Errance, coll. « Hespérides », 256 p.

Petrognani S. et SAUVET G. 2012. La parenté formelle des grottes de Lascaux et de Gabillou est-elle formellement établie ?, Bulletin de la Société préhistorique française, t. 109, $\mathrm{n}^{\circ} 3, \mathrm{p} .441-455$.

PIGEAUD R. 2003. Le panneau principal de la grotte ornée Mayenne-Sciences (Thorignéen-Charnie, Mayenne) : une composition triangulaire organisée, Revue archéologique de l'Ouest, vol. 20, p. 13-31.

PigEAUD R. 2004 (avec la collaboration de M. Bouchard et É. Laval). La grotte ornée Mayenne-Sciences (Thorigné-en-Charnie, Mayenne) : un exemple d'art pariétal d'époque gravettienne en France septentrionale, Gallia Préhistoire, 46, p. 1-154.

PigeAUD R. 2005. Immédiat et successif: le temps de l'art des cavernes, Bulletin de la Société préhistorique française, t. $102, \mathrm{n}^{\circ} 4$, Actes du $129^{\mathrm{e}}$ Congrès des Sociétés historiques et scientifiques, Besançon, 19-24 avril 2004, p. 813-828.

PIGEAUD R. 2013. Derrière la paroi : portrait robot de l'artiste paléolithique, in M. GROENEN (dir.), Expressions esthétiques et comportements techniques au Paléolithique, Oxford, BAR International Series 2496, p. 87-93.

Pigeaud R., Berrouet F., Bougard E., Paitier H., Pommier V., Bonic P. 2012 (avec la collaboration de M.-D. PINEL, M.-L. LATREILlE et A. REDOU), La grotte du Sorcier à SaintCirq-du-Bugue (Dordogne, France): nouvelles lectures. Bilan des campagnes 2010 et 2011, Paleo, n' 23, p. 223-248.

Pigeaud R., Hinguant S., Rodet J., Devièse T., Dufayet C., Heimlich' G., Mélard N., BETTON J.-P., BONIC P. 2010. The Margot Cave (Mayenne): a new Palaeolithic sanctuary in West France, in K. SHARPE et J. ClOTTES (éd.), European Cave Art, BAR International Series 2108, p. 81-92.

Pigeaud R., Hinguant S., PAitier H., POMmier V., Bonic P. (avec la collaboration de M.D. Pinel, F. Berrouet et A. Redou) 2012. La grotte Margot (Thorigné-en-Charnie, Mayenne) : un sanctuaire complexe aux influences multiples, Préhistoire, Arts $\mathcal{E}$ Sociétés, t. LXVII, p. 81-101.

SAUvet G. et TOSEllo G. 1998. Le mythe paléolithique de la caverne, in F. SACCO et G. SAUVET (dir.), Le propre de l'Homme, psychanalyse et préhistoire, Lausanne, éd. Delachaux et Niestlé, coll. " Champs psychanalytiques », chap. 3, p. 55-90.

TAUXE D. 2007, L'organisation symbolique du dispositif pariétal de la grotte de Lascaux, Préhistoire du Sud-Ouest, n 14, p. 177-266.

VIALOU D. 1982, Niaux, une construction symbolique magdalénienne exemplaire, Ars Praehistorica, $\mathrm{n}^{\circ} 1$, p. 19-45.

VIALOU D. 1983, Art pariétal paléolithique ariégeois, L'Anthropologie, t. 87, n 1, p. 83-97.

VIALOU D. 1986, L'art des grottes en Ariège magdalénienne, XXII ${ }^{\mathrm{e}}$ supplément à Gallia Préhistoire, Paris, CNRS éd., 432 p.

VIALOU D. 1989, L'art paléolithique, un message codé, in J.-P. MOHEN (dir.), Le Temps de la Préhistoire, Paris-Dijon, coédition Société préhistorique française / Éditions Faton, p. 198203. 


\section{Illustrations}

Figure 1: Grotte de Niaux et Réseau Clastres (Ariège) : plan avec la localisation des représentations. D'après Clottes, 2010.

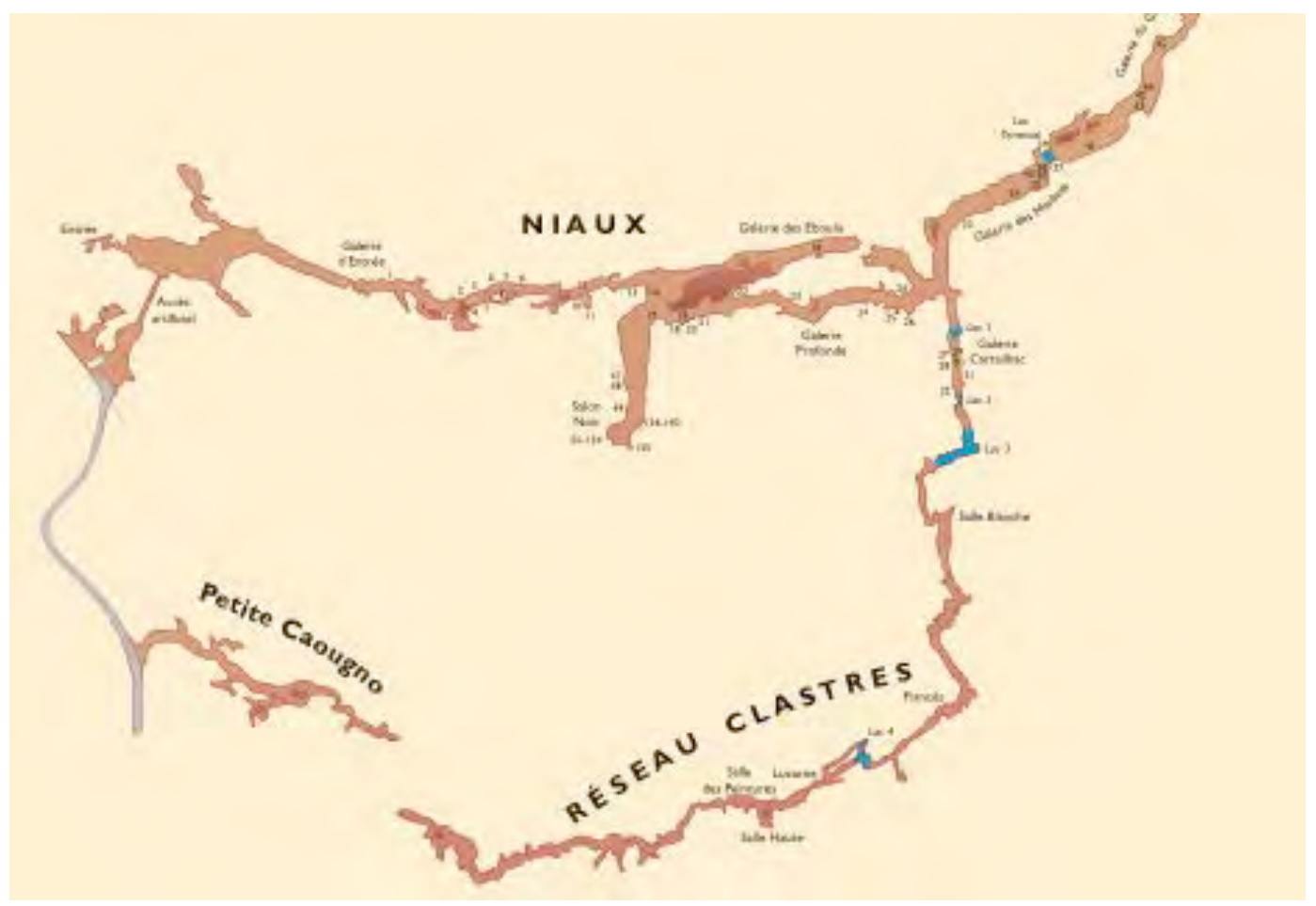

Figure 2 : Grotte de la Tête-du-Lion (Ardèche) : aurochs, encornure de bouquetin et lignes de points rouges et jaunes. Photo Romain Pigeaud.

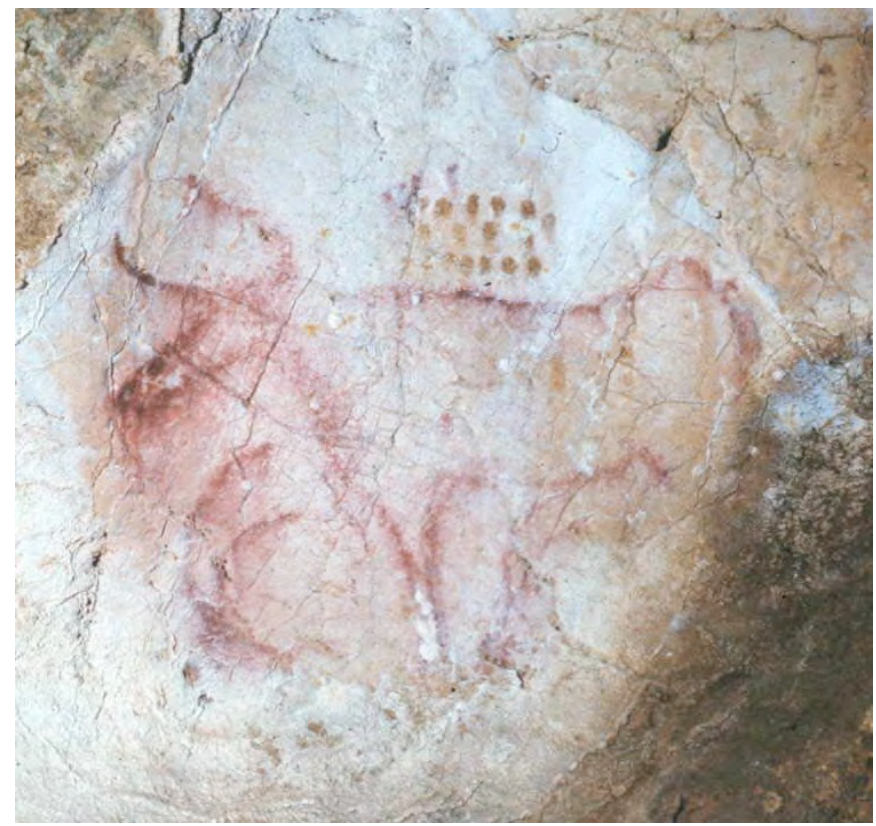


Figure 3: Grotte Mayenne-Sciences (Mayenne): panneau principal avec cheval et mammouth affrontés, deuxième cheval et signe triangulaire ovalisé. Photo Hervé Paitier. Avec l'accord des communes propriétaires.

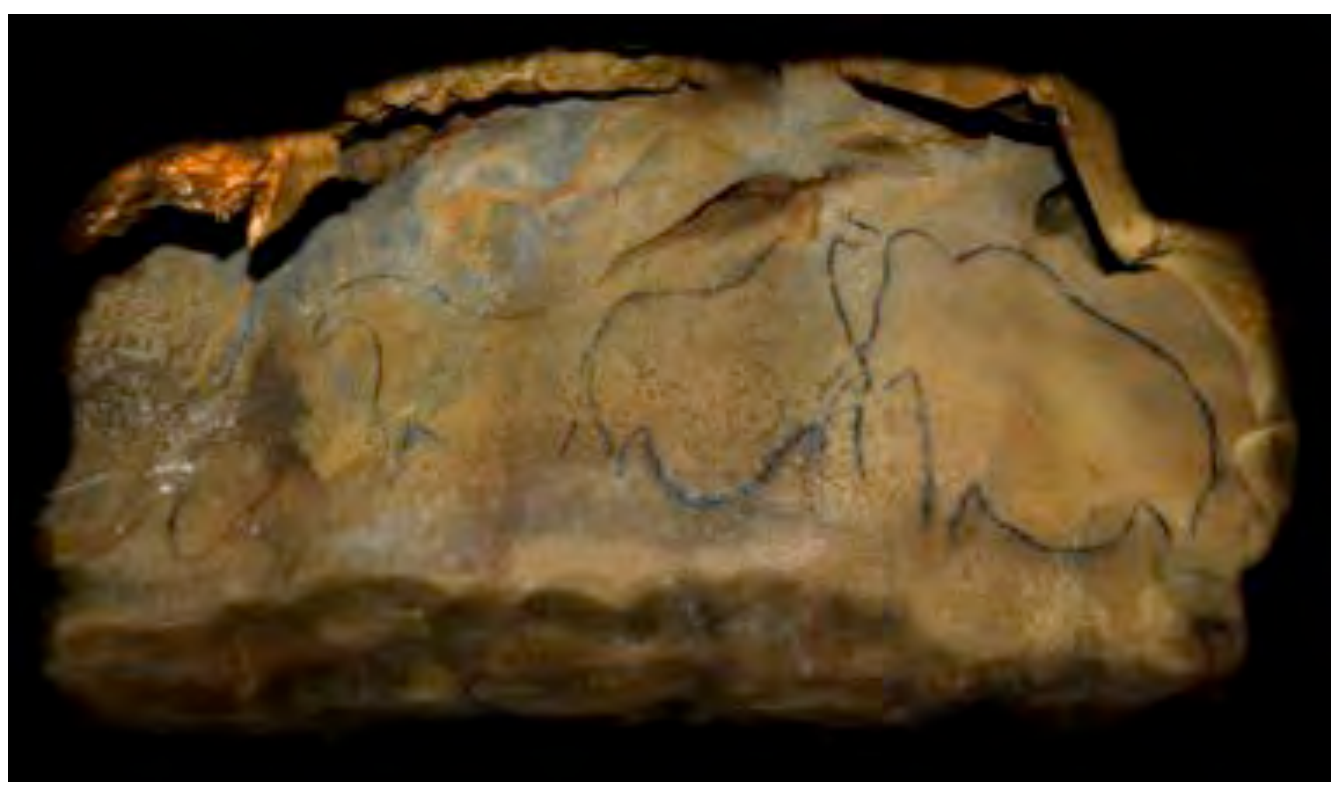


Figure 4: Grotte du Sorcier (Dordogne): la coupole du «Sorcier». En jaune, les arrachements de paroi. En blanc, les efflorescences de gypse. En rouge, les détériorations modernes.

Relevé Romain Pigeaud, avec la collaboration de Florian Berrouet et d'Estelle Bougard.

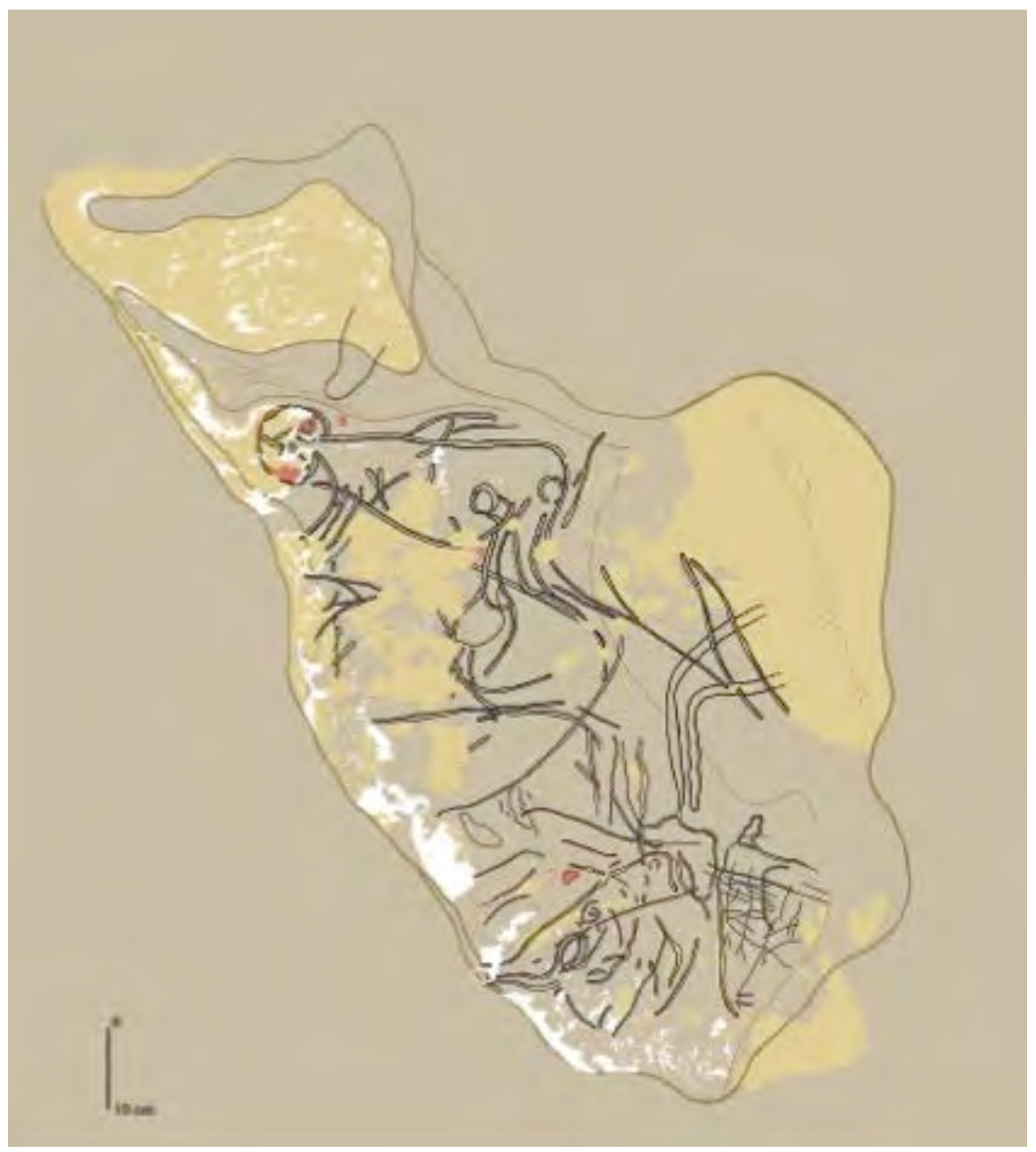


Figure 5 : Grotte de Lascaux (Dordogne) : le diverticule axial avec aurochs, chevaux, cerf et signes. Relevés Norbert Aujoulat. D’après Aujoulat, 2004.

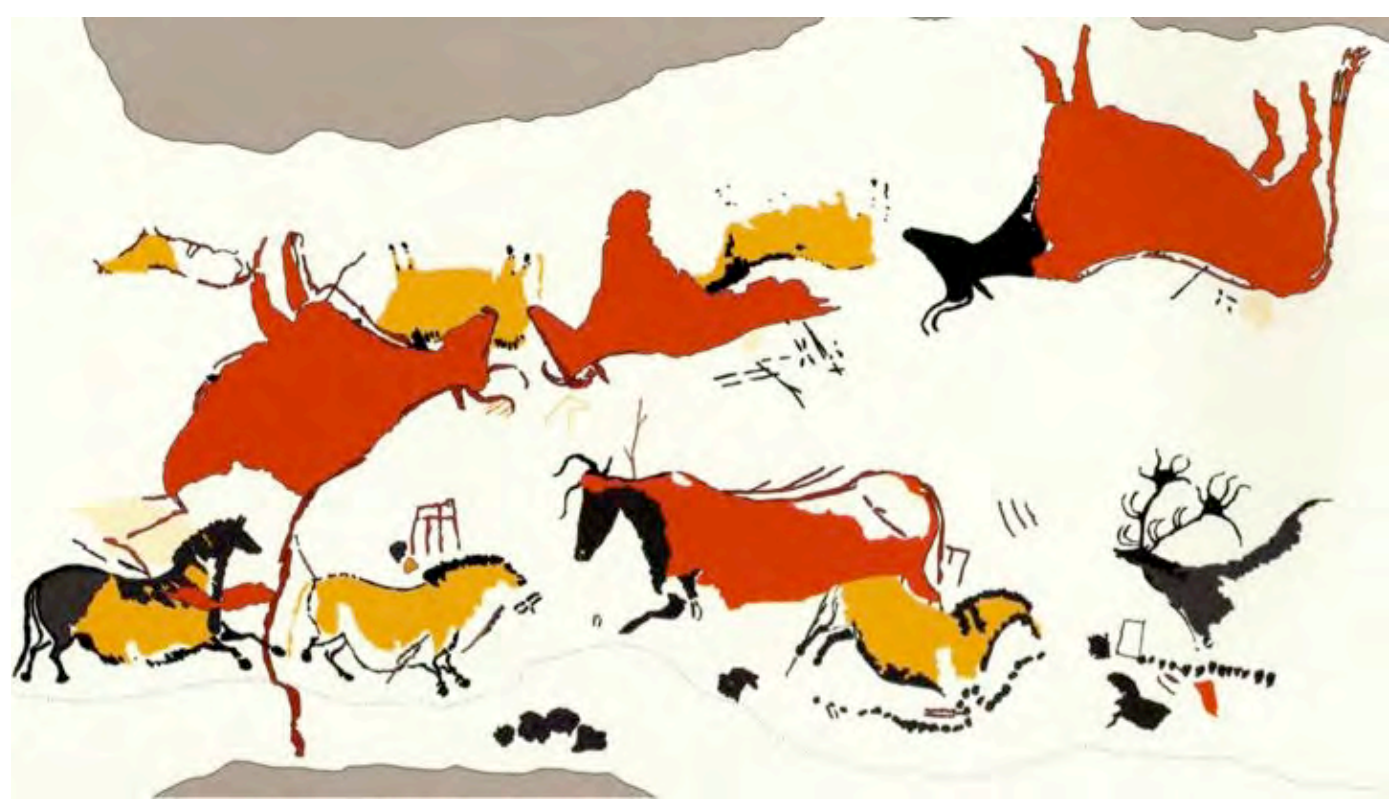

Figure 6 : Grotte Chauvet-Pont-d'Arc (Ardèche) : panneaux des lions de la Salle du Fond. Photo MCC- D.R.A.C. Rhône-Alpes.

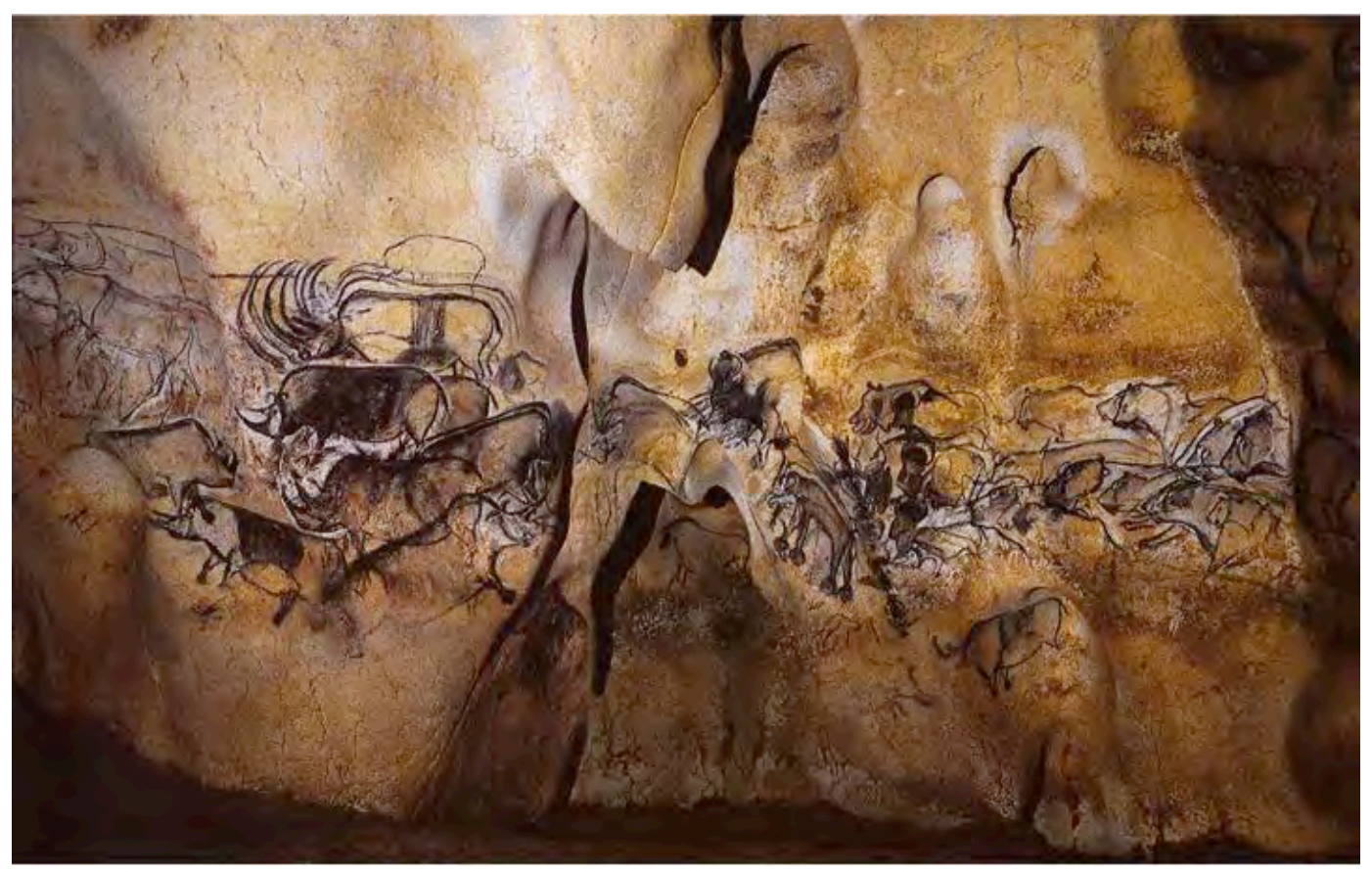


Figure 7: Grotte de La Baume Latrone (Gard) : panneau principal avec félin central et mammouths. Photo Alain Dubouloz. D'après Azéma, 2011.

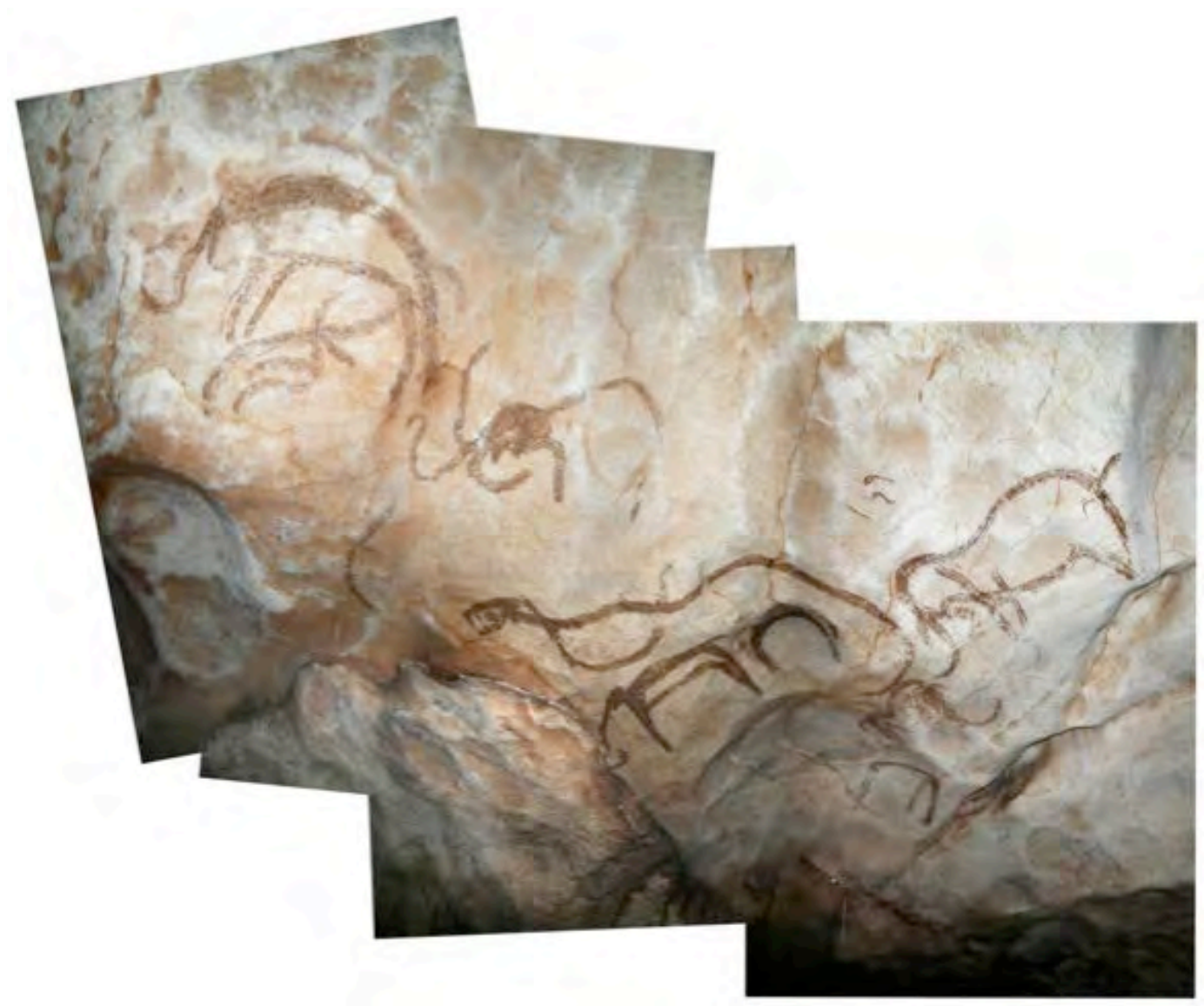


Figure 8: Grotte Margot (Mayenne): gravure d'un rhinocéros utilisant une fissure naturelle. Photo Hervé Paitier, relevé Alice Redou et Florent Duval.

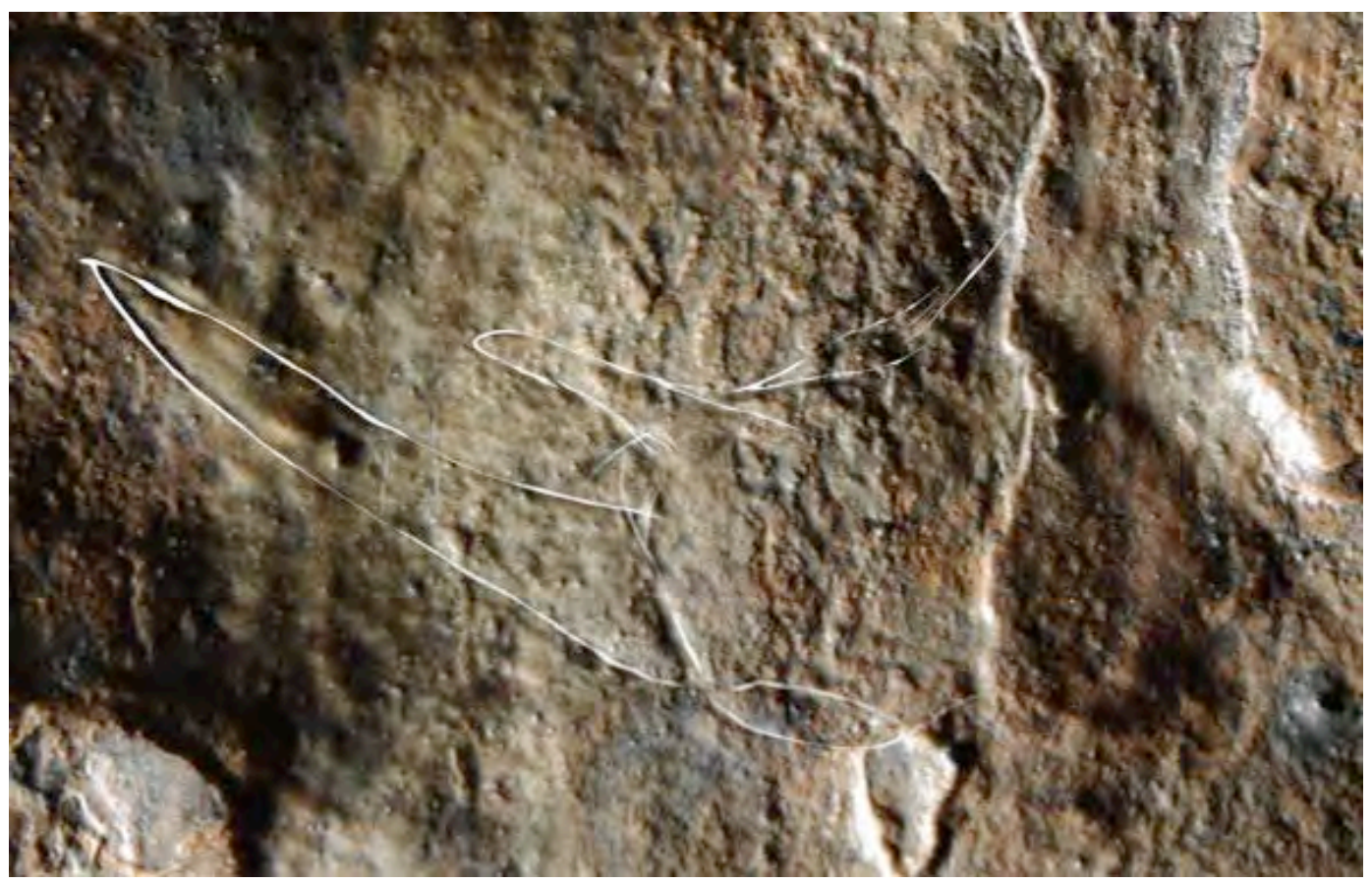


Figure 9: Grotte du Sorcier (Dordogne) : l'autre côté de la coupole du «Sorcier». En jaune, les arrachements de paroi. En blanc, les efflorescences de gypse. En rouge, les détériorations modernes. Relevé Romain Pigeaud et Florian Berrouet.

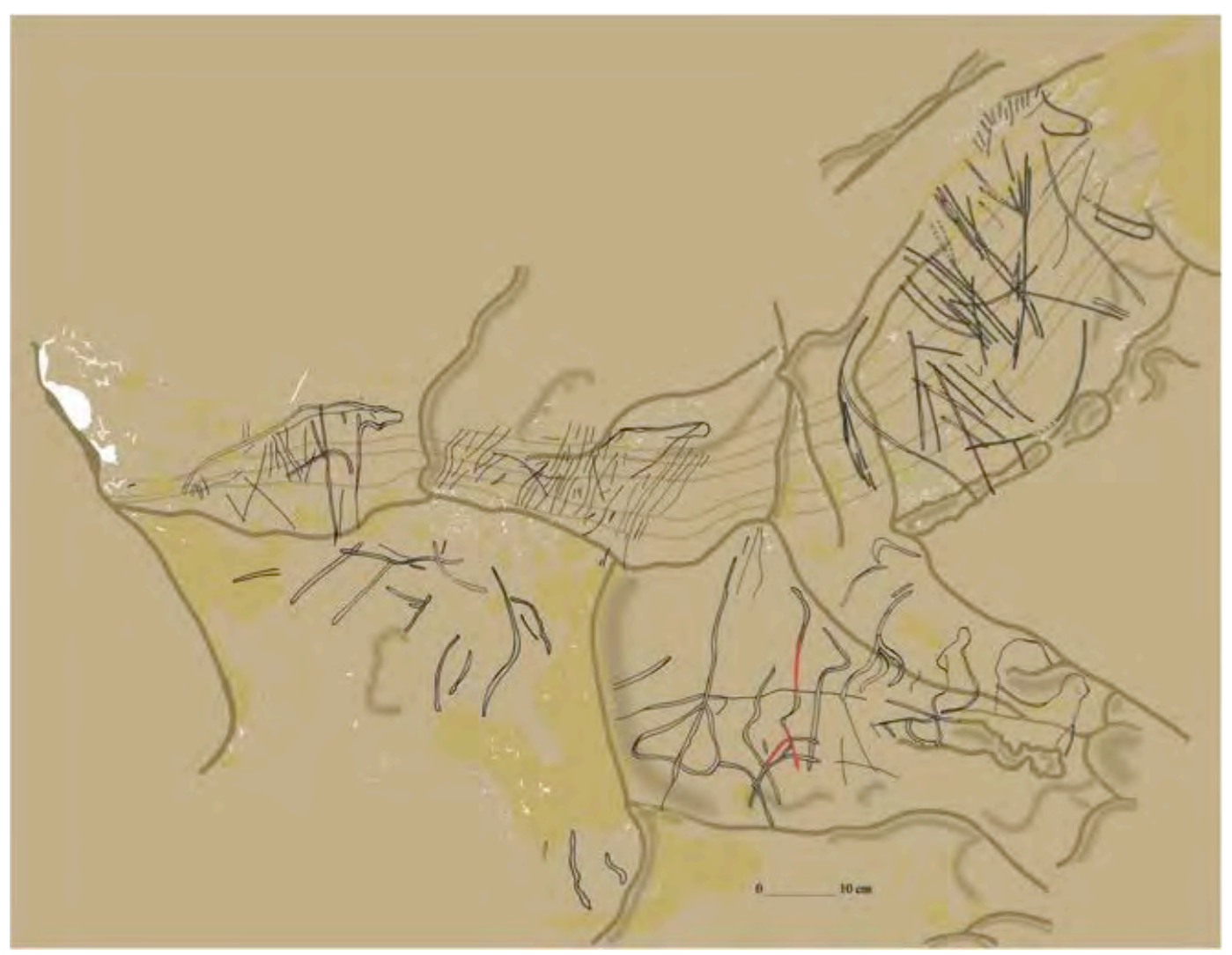


Figure 10: Grotte du Pergouset (Lot) : vulve et animaux schématiques. Relevé Michel Lorblanchet. D'après Lorblanchet, 2001.

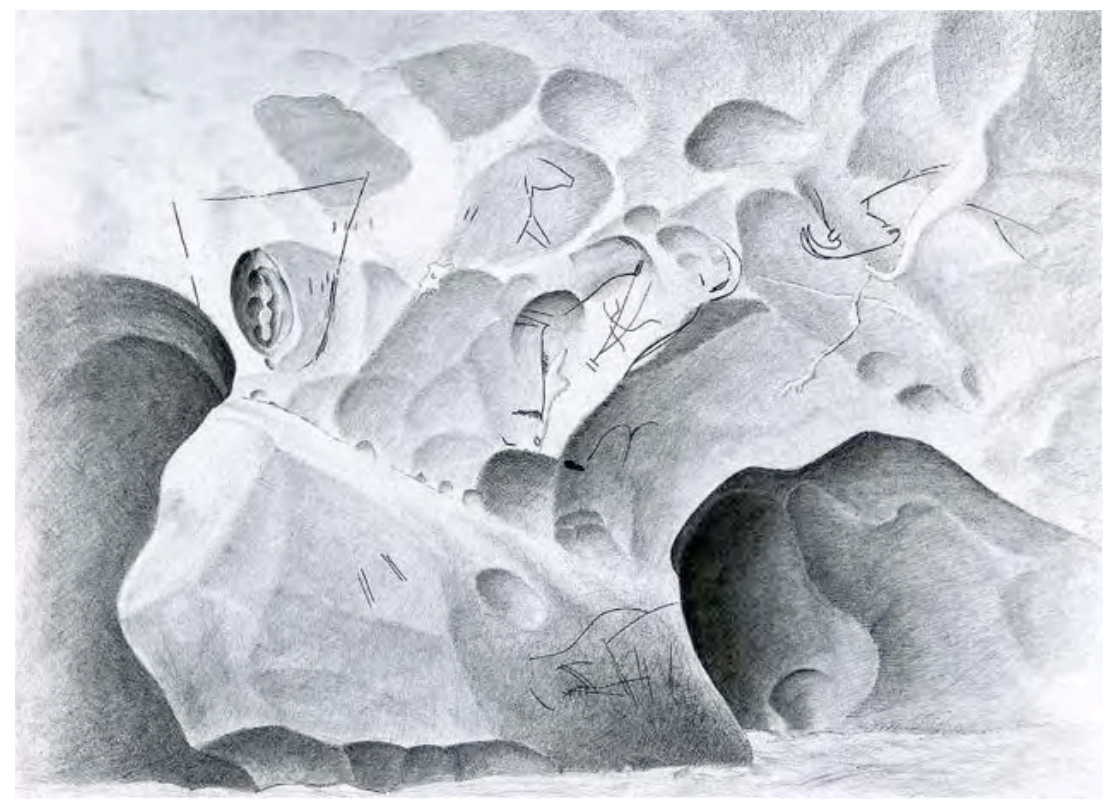

Figure 11: Grotte Margot (Mayenne) : vulve gravée de la galerie du Chêne Pétrifié. Relevé Romain Pigeaud.

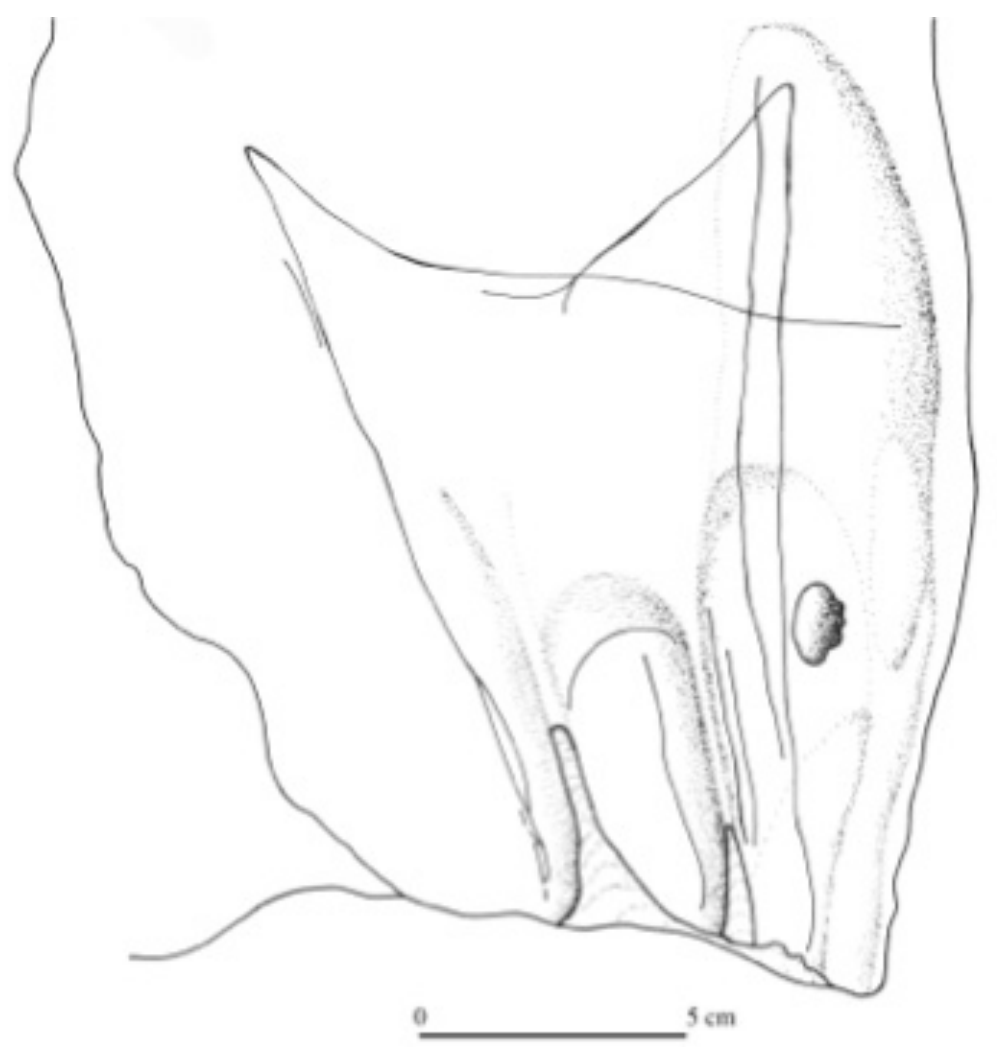




\title{
Les mégalithes et leur insertion sémantique dans le paysage. Langage d'ancêtres
}

\author{
Primitiva BUENO RAMÍREZ, \\ Professeur de Préhistoire, Université de Alcalá, Espagne \\ Rodrigo de BALBÍN BEHRMANN, \\ Professeur de Préhistoire, Université de Alcalá, Espagne \\ Rosa BARROSO BERMEJO \\ Maître de Conférences de Préhistoire, Université de Alcalá. Espagne
}

\author{
Extrait de : Olivier BUCHSENSCHUTZ, Christian JEUNESSE, Claude MORDANT \\ et Denis VIALOU (dir.), Signes et communication dans les civilisations de la parole, Paris, \\ Édition électronique du CTHS (Actes des congrès des sociétés historiques et scientifiques), 2016. \\ Cet article a été validé par le comité de lecture des Éditions du CTHS dans le cadre de la publication \\ des actes du $139^{\mathrm{e}}$ Congrès national des sociétés historiques et scientifiques tenu à Nîmes en 2014
}

\section{Résumé}

La présence de grandes pierres dans le paysage des constructeurs de mégalithes a été vérifiée archéologiquement en Bretagne française. Les recherches ont porté sur un noyau très important qui peut se dater au cours $d u V^{e}$ millénaire avant notre ère. Récemment, les travaux archéologiques développés dans la péninsule Ibérique ont montré une richesse inédite qui permet de réfléchir sur le rôle des grandes pierres par rapport aux mégalithes. L'inventaire des grandes pierres dans la péninsule Ibérique se rapproche chaque fois davantage des inventaires mieux connus de Bretagne. Diverses situations existent: cercles ou alignements qui se transforment en dolmens, menhirs solitaires ou stèles-menhirs qui sont la première pierre d'un dolmen représentent toute une série de preuves qui nous parlent de la complexité et de la variabilité de situations dans lesquelles les vieilles grandes pierres ont été les précurseurs ; pas seulement dans une première phase de construction des mégalithes, mais pendant tout le développement du mégalithisme.

Ces marques gravées et peintes sur les roches et grandes pierres représentent les témoignages de marquages de territoires occupés dès le Paléolithique supérieur dans la péninsule Ibérique.

Mots-clés: Mégalithisme. Menhirs. Stèles. Art mégalithique. Bretagne. péninsule Ibérique. Europe. Paléolithique supérieur. Néolithique.

\begin{abstract}
Large stones have been identified in the Megalithic landscape of Brittany. J. L'Hegouach and S. Cassen's research has focalised on an important core of these monuments dating from the $5^{\text {th }}$ millennium BC. More recent studies carried out in the Iberian Peninsula have highlighted the unprecedented wealth of data from this area which allows us to reflect on the function of these large stones in relation to the megalithic structures. This inventory underlines the similarities between the Iberian Peninsula and Brittany. Stone circles or lines can transform into dolmens, isolated menhirs or steles become the founding stone of a dolmen. This represents proof of the complexity and the varying situations in which these ancient stones are the forerunners of megalithic construction into which they are integrated for the duration of the megalithic phase.

The engravings and paintings on the rocks and large stones constitute markers of territories populated since the Late Palaeolithic in the Iberian Peninsula. (traduction Rebecca Peake)
\end{abstract}


C'est une notion commune que de présenter l'élévation de grandes pierres qui caractérisent les paysages de la façade atlantique durant la préhistoire récente comme la première preuve de la domestication du paysage (Bradley 2000, Bueno et al. 2003). Personne ne doute que ces grandes pierres soient des symboles créés par les groupes humains, puisque ce sont des manifestations totalement artificielles situées dans des espaces choisis par leurs constructeurs. Leur caractère le plus significatif porte sur la volonté de visibilité ; leur présence dans le paysage évoque l'intérêt de ceux qui les ont transportées, décorées (si c'est le cas) et levées, pour marquer ces territoires à partir de signaux faciles à percevoir. On accepte aussi le fait que les pierres en plein air représentent des figures humaines, comme une matérialisation des ancêtres (Bueno et al. 2008, Pearson et Ramilosonina 1998, L'Helgouach 1983). Les études menées dans d'autres régions, ainsi que sur les données péninsulaires, confirment la relation des décors géométriques de certains menhirs avec ceux des supports mégalithiques et des restes mobiliers contemporains à caractère anthropomorphe (Bueno 1992, 1995 ; Bueno et Balbín 1994 ; Bueno et al. 2005).

Les symboles gravés, peints et sculptés sur les structures funéraires et les installations en plein air, offrent un répertoire visible de référence étudié par les chercheurs européens. L'inventaire de E. Shee Twohig (1981) résume les points fondamentaux de ce type d'analyse jusqu'à la fin $\mathrm{du} X \mathrm{X}^{\mathrm{e}}$ siècle et il signale les similitudes et les différences, les relations entre les différentes aires de l'Europe atlantique.

L'épanouissement du système serait lié aux groupes mésolithiques côtiers et il se localise tout spécialement sur la façade nord occidentale de la France, plus particulièrement en Bretagne (Migdley 2013). La transition ou la relation entre les grandes pierres de plein air et leur inclusion dans des structures fermées à finalité funéraire constituent des paramètres significatifs pour valoriser les liens symboliques entre les plus anciens systèmes de signalisation des groupes humains et ceux qui s'associent au mégalithisme atlantique. Les menhirs furent assimilés, à l'origine des mégalithes, à la première étape de construction (Calado 1997). En Bretagne, cette première phase a été décrite comme une authentique damnatio (L'Helgouach 1983), ou au moins, en admettant cette idée d'une antiquité originelle des menhirs (Cassen 2005), comme un processus d'actions consécutives : en premier, les menhirs en plein air, ensuite les dolmens.

Dans ce texte, on développera la présentation des données relatives à la présence de grandes pierres dans le mégalithisme de la péninsule Ibérique. Cette approche prend un intérêt majeur avec la reconnaissance du rôle du mégalithisme dans des territoires où une utilisation très ancienne est attestée par des roches ornées dès le Paléolithique supérieur. Dans ce contexte, cette lecture est une hypothèse, applicable à d'autres territoires du mégalithisme européen où l'on commence à percevoir la possibilité d'identifier d'amples séquences chronologiques d'occupations antérieures aux mégalithes (Loveday 2012, Moullec et al.1996).

Actuellement, de nombreux éléments ont changé dans la péninsule Ibérique. Des projets de prospection intensive ont été menés dans toute la partie occidentale avec d'excellents résultats, notamment pour la localisation des roches décorées du Paléolithique supérieur (Balbin 2009). On peut confirmer que la définition de paysages humanisés grâce aux signes visibles sur les roches fait partie des systèmes culturels des groupes des anciens chasseurs-cueilleurs. Leur continuité chronologique est un fait tant pour les roches en plein air que pour les contextes souterrains, ou sur l'art mobilier. Les sites marqués par les chasseurs du Paléolithique supérieur sont ensuite reconnus et signalés comme particuliers par des groupes mésolithiques, néolithiques et chalcolithiques (Balbin et Bueno 2009, Bueno 2009, Bueno et Balbín 2009, Bueno et al. 2007a). Les ensembles du Douro, du Taje et du Guadiana représentent d'excellents cas d'étude.

Un autre ensemble de nouveautés est propre au mégalithisme ; il concerne en particulier la diversité des architectures mégalithiques, leur chronologie similaire dans différents secteurs géographiques, leur ample développement jusqu'au-delà du $\mathrm{III}^{\mathrm{e}}$ millénaire cal 
BC et leur notable extension dans les territoires intérieurs européens (Bueno et al. 2013a, p. 490 ; Furholt et Muller 2011 ; Laporte 2010). Avec le renouvellement des systèmes de datation $\mathrm{C} 14$ des mégalithes ibériques, à partir de datations directes sur des peintures mégalithiques ou sur ossements humains par AMS, la péninsule Ibérique se place en position centrale pour réfléchir à certaines questions développées dans ce texte (Steellman et al. 2005, Carrera et Fábregas 2002, Bueno et al. 2007, Scarre et al. 2003).

Le renouveau méthodologique dans l'étude des mégalithes péninsulaires a produit des nouveautés intéressantes des années 1990 jusqu'à nos jours (Carrera 2011). Certaines preuves montrent les rapprochements des plans et des techniques dans tout l'ensemble atlantique (Bueno et Balbín, 1992, 2002 ; Calado, 1997, 2002), et notamment avec la Bretagne française, ainsi que la présence de matières premières d'origine péninsulaire dans des mégalithes bretons (Herbault et Querré 2004). D'autres données soulignent le rôle de la façade nord péninsulaire et de la voie de l'Ebre pour expliquer certaines productions symboliques attestées dans le Sud et sur la façade occidentale française (Bueno et al. 2009a). Cependant, un des paramètres les plus intéressants est la possibilité d'établir des relations entre les figurations de plein air et celles de l'intérieur des monuments (Bueno et Balbín 2000 ; Bueno et al 2004, 2010a, 2006, 2013b), ce qui constitue un argument unique pour contribuer à fixer les règles symboliques des constructeurs de mégalithes en Europe. Peintures, gravures en abris-sous-roche et sur roche en plein air s'ajoutent aux stèles et menhirs.

L'association traditionnelle entre les menhirs, les stèles et les premières phases de construction des mégalithes compte maintenant d'intéressantes références à partir des données péninsulaires. Les pierres dressées en plein air s'intègrent dans un système complexe d'insertion en contexte funéraire qui va de pair avec l'érection de nouveaux ensembles de pierres en plein air. Ainsi, les processus stèles-menhirs / monuments mégalithiques ne sont pas exclusifs de la phase la plus ancienne de la construction des sépultures. Ils constituent la base de la génération de passés successifs, initiés par des pierres d'une grande signification ancestrale qui se transforment, de monuments de plein air, en monuments fermés et qui peuvent à leur tour générer de nouveaux sépulcres ou, éventuellement, de nouveaux monuments en plein air (Bueno et al. 2005, 2007b). Si la «biographie» des sépulcres s'avère un argument d'un intérêt extraordinaire pour analyser le rituel des ancêtres, l'histoire des pierres elles-mêmes apporte des preuves de leur valeur et de leur signification individuelle.

Pour aborder les lectures de ce type, des protocoles ont été développés autour de la documentation relative à l'Art mégalithique dans la péninsule Ibérique et ils ont permis d'obtenir des résultats significatifs. On a pu démontrer l'existence d'un fort pourcentage de stèles et menhirs réutilisés dans des monuments ibériques. Cette situation se rapproche $\mathrm{du}$ cas breton, mieux connu, et révèle des phénomènes très répétitifs de transformations de monuments durant la période évoluée du mégalithisme. La seconde moitié $d u \mathrm{IV}^{\mathrm{e}}$ millénaire et la première moitié $\mathrm{du} \mathrm{III}^{\mathrm{e}}$ se caractérisent par des nécropoles installées autour de mégalithes créés à partir de stèles et de menhirs provenant de monuments antérieurs; ce phénomène s'accentue dans le Sud de la péninsule. Cette confirmation a été obtenue à partir de différents travaux de terrain qui placent cette région de la péninsule Ibérique dans une position optimale pour la mise en valeur des interactions symboliques au sein du mégalithisme de la façade atlantique européenne (Bueno et al. 2007b, 2008, 2010b, 2013c).

\section{Les messages à l'extérieur des mégalithes: les pierres comme matérialisation visible de l'emplacement des groupes humains.}

Les pierres levées représentent les plus grandes manifestations artificielles de l'intérêt des groupes humains pour se rendre visibles. En Europe, certaines données signalent que ces créations auraient pu être réalisées en bois dans des périodes anciennes (Rust 1943), mais 
leur développement a été plus notable à partir du Néolithique. Durant les années 1970 et 1980, les fouilles en Bretagne ont signalé le rôle des menhirs qui auraient eu une plus grande importance que celle admise aujourd'hui (Briard et al. 1995). La pièce décorée en bois de Maerdy (Pays de Galles), récemment documentée, indique l'existence de ces poteaux avec décorations datées $\mathrm{du} \mathrm{V}^{\mathrm{e}}$ millénaire cal BC (Jones 2013). La technique et la thématique de ses décors relient ces bois ornés à des répertoires bien connus sur les supports en pierre des mégalithes européens (Bueno et al., 2015) (fig. 1).

Les menhirs ont été traditionnellement considérés comme une production culturelle atlantique, avec une notable représentation dans l'aire bretonne. Mais les études réalisées dans la péninsule Ibérique et dans d'autres aires du continent enrichissent la lecture de ces pierres très visibles, fréquemment décorées (Bénétau 2000, Foster 2013, D’Anna et al. 2012), dans les territoires mégalithiques.

Dans la péninsule Ibérique, la position occidentale de l'Alentejo a été considérée comme la preuve de son rôle de noyau originel (Calado 2002). Mais, dans la situation actuelle, un ample inventaire de menhirs avec des contextes archéologiques bien datés témoigne autant de l'existence des phases les plus anciennes que de la continuité dans l'édification de grandes pierres au cours des $\mathrm{IV}^{\mathrm{e}}$ et $\mathrm{III}^{\mathrm{e}}$ millénaires cal BC. En Catalogne, en Galice, en Andalousie et à l'intérieur de la péninsule, des dates du $\mathrm{V}^{\mathrm{e}}$ millénaire cal BC apparaissent pour certains sites et parmi ceux-ci, nombre de menhirs ont l'avantage supplémentaire d'être associé à l'installation des premiers agriculteurs (Bueno et al. 2007b, 2009a).

Il paraît probable que certains monuments mégalithiques se situent autour de menhirs emblématiques et très visibles; cela rend possible des séquences d'agrégation, comme c'est le cas du menhir du Cabezo, à Alcántara. Les datations du $\mathrm{IV}^{\mathrm{e}}$ et $\mathrm{III}^{\mathrm{e}}$ millénaire cal BC de la nécropole de dolmens à proximité du menhir du Cabezo suggèrent que celui-ci avec certaines céramiques imprimées dans le remplissage de la fosse - aurait été implanté antérieurement. Malheureusement la chronologie C14 ne fut pas probante (fig. 2).

La décoration élaborée en bas-relief confirme la forte pertinence de ce type de gravures sur les menhirs et stèles de plein air, ce qui se retrouve autant dans la péninsule Ibérique qu'en Bretagne. Il est possible que cette technique soit liée à cette intention de visibilité décorative d'un monolithe, parfois de dimensions considérables. Sa position par rapport au soleil aiderait à ce que le bas-relief soit perceptible peu à peu, favorisant une lecture au cours de laquelle la lumière et le temps sont des facteurs à prendre en compte. Par ailleurs, le fait que le bas-relief présente les preuves d'une telle ancienneté valide son ancrage dans des références techniques de longue durée pendant la Préhistoire.

Dans l'intérieur de la péninsule Ibérique, les données relatives à l'implantation des menhirs sont de plus en plus nombreuses. Récemment, les découvertes les plus importantes se localisent en Andalousie, où la recherche sur ces témoins a été intensifiée. Certains cas montrent des relations évidentes avec le scénario de l'art atlantique. C'est le cas, en particulier, de Arroyo de Sileras ou des pierres récemment trouvées à Belalcazar, dans la province de Cordoue. D'ailleurs, à Cordoue, il y a quelques années, l'étude de la construction du dolmen de Casas de Don Pedro a montré que sa réalisation s'est faite à partir de deux menhirs préexistants (Gavilán et Vera 2005). À Cadiz, l'association avec des nécropoles mégalithiques est très significative, de même que leur relation avec les voies de transhumance (Bueno et al. 2010c). Cette relation entre la localisation de certains menhirs et ces passages a été proposée par les chercheurs qui ont travaillé sur l'ensemble de menhirs de la Meseta Nord, entre Palencia et Burgos. Les datations C14 permettent de fixer leur installation dans les premiers moments du $\mathrm{IV}^{\mathrm{e}}$ millénaire cal $\mathrm{BC}$, avec une survivance significative dans les périodes plus récentes, au $\mathrm{III}^{\mathrm{e}}$ millénaire cal $\mathrm{BC}$ (Moreno et Delibes 2007).

Ainsi, nous nous trouvons dans une situation très similaire à celle des monuments mégalithiques au sens propre. Les chronologies coïncident entre elles, ce qui éloigne les 
possibilités d'en déterminer le noyau d'origine. Comme c'est le cas pour les mégalithes, ces menhirs signalent plutôt la possibilité de chronologies plus anciennes pour l'érection de ces grandes pierres et celle d'une notable extension du système coïncidant avec une complexification résidentielle plus forte des premiers agriculteurs. L'idée d'associer l'image des poteaux en bois des villages mésolithiques du Nord de l'Europe, avec les cas postérieurs d'éléments organiques occasionnellement décorés comme celui de Maerdy et les menhirs en pierre est très séduisante. Ces derniers se placeraient dans la continuité d'une tradition qui aurait eu une plus grande expression sur des matériaux organiques qui n'ont pas perduré jusqu'à nos jours.

\section{«Biographie des pierres»}

Les développements précédents appuient notre argumentation sur l'hypothèse de la valeur individuelle de chacune des pièces qui composent les mégalithes. L'association de ces pierres avec des images anthropomorphes est aisée, de sorte que proposer des liens avec les images d'ancêtres s'avère une hypothèse séduisante. Dans cette optique, on a proposé de définir la position de certains menhirs et stèles réutilisés dans la construction de mégalithes comme celle $\mathrm{d}^{\prime}$ " ancêtres fondateurs ». Ce sont ces pierres de grande valeur symbolique, et probablement accumulatrices du passé, qui sont érigées en tant que premières pierres de certains monuments mégalithiques (Bueno et al. 2005, 2008, 2014).

La « biographie » des sépulcres représente un cadre d'étude très intéressant, mais certains protocoles doivent être intégrés pour conduire cette analyse de la «biographie » des pierres, en considérant chacune d'elles comme la matérialisation d'un ancêtre dont la première version aurait pu être beaucoup plus ancienne que le mégalithe dans lequel se fait son emplacement définitif.

Avec cet objectif, notre protocole de documentation des mégalithes propose une analyse détaillée, support par support. Chacun d'entre eux est considéré comme un panneau et analysé comme tel. De cette manière, nous avons pu identifier des tailles spécifiques qui caractérisent certains supports, des décors peints ou gravés, qui se situent dans les zones non accessibles une fois le sépulcre construit. Avec les études détaillées des matières premières et du contexte archéologique, ces données contribuent à déterminer la grande fréquence - en termes quantitatifs -, de stèles et menhirs réutilisés dans les mégalithes ibériques. Cette voie d'analyse a mis en évidence tout un répertoire de réutilisations dans le mégalithisme ibérique qui atteint celui bien connu dans d'autres contextes européens ; elle révèle que cette systématisation est beaucoup plus commune qu'il était admis jusqu'à présent.

En effet, les protocoles appliqués dans l'étude de l'Art mégalithique présentaient un panorama de données qui, jusqu'à très récemment, était impensable pour la péninsule Ibérique. Ces résultats ont été obtenus en prenant en compte la répartition des menhirs dans tout le territoire, leur association avec des nécropoles et des chemins et, bien sûr, la possibilité d'obtenir des références datables à partir des réutilisations au sein des mégalithes ou à partir de l'étude des contextes d'implantation de ces pierres dans des ensembles de plein air. La variabilité chronologique que nous avons signalée antérieurement constitue une base pour aborder l'étude de situations diverses dans lesquelles les menhirs sont inclus dans des mégalithes qui, par ailleurs, peuvent être signalés ou accompagnés par des menhirs. L'expression "mégalithisme non funéraire ", utilisée pour regrouper les données de menhirs en plein air, a perdu beaucoup de valeur au vu de la complexité des situations qui peuvent être documentées actuellement.

Les fouilles menées durant les dernières années permettent de confirmer de longues trajectoires dans les agrégations de menhirs en plein air qui se manifestent par l'augmentation du nombre de blocs, la réfection des plans et, en somme, qui révèlent une complexité tout à fait comparable à celle des monuments fermés, c'est-à-dire, à celle des 
mégalithes. Ceci devient visible autant dans les données obtenues à partir des cromlechs portugais (Gomes 1994; Calado 1997, 2002), que dans les remarquables monuments atlantiques (Darvill 1997), ou dans les alignements méditerranéens (D'Anna et al. 2012). Certains de ces menhirs de plein air furent utilisés pour signaler des zones potentielles de nécropoles.

L'abondance des sites de plein air dans la péninsule Ibérique constitue une caractéristique très intéressante, que l'on ne traitera pas ici. On soulignera seulement que certains travaux ont permis d'obtenir des références pour les monuments desquels provient une partie des menhirs réutilisés pour la construction de dolmens. Pour la première fois en Europe, non seulement on peut signaler la présence de menhirs ou de stèles réutilisées, mais on peut apporter des preuves archéologiques concernant les monuments d'où proviennent ces éléments.

La position de certains menhirs fut à l'origine des sépulcres mégalithiques et comme on l'a signalé antérieurement, leur rôle comme "Ancêtres fondateurs » devient assez convaincant. Les menhirs ou stèles constituent la référence idéologique sur laquelle s'établissent de nouveaux passés avec la construction d'un sépulcre qui hébergera d'autres ancêtres. Les données s'accroissent et plusieurs monuments se créent autour d'une stèle ou d'un menhir préexistant. C'est le cas du dolmen de Telhal, à Evora, signalé par une stèle-menhir réutilisée. Il est certain qu'une étude exhaustive augmenterait cet inventaire, car certains documents du mégalithisme évolué (entre le $\mathrm{IV}^{\mathrm{e}}$ et le $\mathrm{III}^{\mathrm{e}}$ millénaire cal BC.) se caractérisent par l'exceptionnelle position, en taille et en forme, de la dalle de chevet. Un réexamen de ces supports, dans l'optique des protocoles énoncés et mis en œuvre précédemment donnerait probablement des résultats fort intéressants (fig. 3).

Dans d'autres cas, le réemploi des monuments offre l'avantage d'apporter, dans le domaine péninsulaire, des références chronologiques qui situent certaines de ces réfections dans le $\mathrm{IV}^{\mathrm{e}}$ et le $\mathrm{III}^{\mathrm{e}}$ cal BC. De bons exemples sont fournis par les dolmens de Pozuelo 3 et 4, construits probablement dans la fourchette $\mathrm{IV}^{\mathrm{e}} / \mathrm{III}^{\mathrm{e}}$ millénaire cal BC sur des alignements préexistants (Bueno et al. 2013b, Linares 2011), ou le dolmen de Llano de la Belleza, réalisé sur un cromlech plus ancien (García Sanjuán et al. 2003). La géophysique authentifie la présence d'un cromlech antérieur, d'où les blocs pour la construction du mégalithe auraient été extraits. Hypothétiquement, un cercle de ce type est l'ensemble de menhirs de Pasada del Abad, très proche du dolmen de Llano de la Belleza cité plus haut (Linares 2011). Un alignement situé à proximité a servi à la construction de Reguers del Seró, en Catalogne, réalisé avec des fragments de stèles. Celles-ci ont fourni des datations obtenues à l'intérieur du monument, de la première moitié du $\mathrm{III}^{\mathrm{e}}$ millénaire cal $\mathrm{BC}$, et la confirmation que les stèles décorées sont contemporaines ou antérieures (López et al. 2009). De plus, un alignement aurait pu être à l'origine des stèles qui composent le dolmen de Os Muiños, en Galice, avec l'avantage que sa peinture originelle a permis d'obtenir des dates C14 directes qui se situent dans la première moitié du IV millénaire cal BC (Carrera 2008).

Les travaux récents dans le dolmen de Soto révèlent une autre possibilité. Certains mégalithes auraient pu être le résultat de la transformation de plus d'un monument préexistant, signalant diverses phases dans «l'acquisition » de ses stèles et menhirs. Celles-ci semblent recueillir différentes générations d'ancêtres dans une même enceinte qui rassemble probablement l'histoire du groupe. On devrait considérer ce type d'hypothèse pour la construction de certains grands monuments atlantiques qui comprennent beaucoup de fragments de pierres réutilisés. Les cas récemment connus en Irlande pourraient s'inscrire dans cette direction, comme les exemples déjà classiques de Gavrinis (Bueno et al. 2013b). Dans le dolmen de Soto, de grands menhirs en pierre de couleurs claires forment la chambre. Sa décoration est majoritairement formée par des cupules et lors de la fouille, on a pu vérifier que des cupules de la base étaient enfouies et cachées dans la fosse d'implantation; ces preuves archéologiques confirment qu'il était gravé avant d'avoir été implanté comme support de la chambre. Pour précisions, les cupules sont les symboles qui caractérisent les menhirs étudiés du cercle de Llano de la 
Belleza, antérieur à la construction du dolmen. De même, les cupules représentent la thématique décorative des menhirs du cercle de La Pasada del Abad (fig. 4).

Le couloir est formé par des pièces plus sombres, plus étroites et basses, avec des décors de tout type. En général, ce sont des formes anthropomorphes évidentes et occasionnellement accompagnées d'armes. En effet, Soto est le monument qui possède une des plus grandes quantités d'armes gravées et peintes de tout l'art mégalithique atlantique. Notre travail crédibilise l'hypothèse que ces pièces auraient pu faire partie d'un alignement précédent et être incluses a posteriori en créant une enceinte majeure pour la sépulture des ancêtres. La présence de peinture rouge et noire enrichit le programme graphique du dolmen, dans lequel il est possible que la peinture s'associe au discours porté (Bueno et al. 2013b.).

Un cas semblable est celui du dolmen de Navalcán où la statue-menhir à l'entrée de la chambre possède une fosse d'implantation qui permet de signaler sa probable situation, à l'origine de l'agrégation de supports qui donnèrent naissance au mégalithe. D'autres supports, comme des menhirs recoupés pourraient provenir d'autres monuments. Les mobiliers recueillis dans le dolmen le situent très probablement entre la deuxième moitié $\mathrm{du} \mathrm{IV}$ et la première moitié du $\mathrm{III}^{\mathrm{e}}$ millénaire cal BC.

La récente étude du dolmen de Lagunita I, en Extremadura, montre des collectes de menhirs ou stèles préexistantes originaires de plus d'un monument. La vieille chambre avec une allée fut détruite pour construire par la suite une petite chambre à espace unique, qui rassembla les stèles d'un autre monument. La grande stèle en relation avec l'espace précédent, et d'autres indices détectés à l'est du mégalithe, suggèrent l'existence d'un monument avec stèles dans le même tumulus. Une construction du IV millénaire a été remplacée par un autre mégalithe au cours du $\mathrm{III}^{\mathrm{e}}$ millénaire qui a ensuite été réutilisé de nombreuses fois jusqu'au I ${ }^{\mathrm{er}}$ millénaire cal BC (Barroso et al. 2012 ; Bueno et al. 2011a).

Ce panorama s'enrichit constamment à mesure que les protocoles d'étude de ce type s'étendent dans les programmes de recherche du mégalithisme péninsulaire. Un bon exemple est celui des récents travaux à Antequera, Málaga. On signale que le dolmen de Menga présente une grande stèle réutilisée recouvrant sa chambre. À Viera, une pièce réutilisée dans la couverture a été mise en évidence. La poursuite des travaux a permis de montrer un processus total de transformation à partir d'un monument préexistant. L'ensemble des pièces composant l'architecture actuelle correspondent à des stèles de plus grande taille, décorées antérieurement de zigzags en relief, du style de celles qui caractérisent les stèles de Reguers ou du Sud de la France. Ces gravures sont peintes en rouge sur les reliefs et présentent, dans les zones basses, des triangles enchaînés dans des ceintures horizontales. Les pièces furent recoupées pour être incluses dans le mégalithe. Le support est totalement peint avec une couche à fort contenu en chaux, et, sur celle-ci sont dessinés des zigzags en rouge et certains en noir. La date récemment publiée du $\mathrm{III}^{\mathrm{e}}$ millénaire cal $\mathrm{BC}$ est une bonne référence qui coïncide avec celles antérieurement citées (Beta-353820, $4090 \pm 30$ BP, 2860-2500 cal BC a 20) (Aranda et al. 2013, p. 241). Les diverses phases de peinture et de gravure sont des arguments indiscutables du processus de transformation de ces édifices, ainsi que des preuves de son entretien constant avec des techniques élaborées dans lesquelles la couleur joue un rôle essentiel (Bueno et al. 2009b, 2013c). 


\section{Pierres et ancêtres. Des références visibles dans le paysage de la préhistoire européenne}

Depuis le Paléolithique supérieur, l'ample registre de pierres décorées de plein air dans la péninsule Ibérique propose des témoins très significatifs pour analyser l'intérêt des groupes préhistoriques à signaler un territoire approprié. Ces symboles étaient connus à partir de leur représentation dans le contexte de grottes, mais leur position dans des gisements de plein air permet d'étendre ce système de signes à tout l'ensemble des groupes de chasseurs du Paléolithique supérieur (Balbin et Alcolea 1999) (fig. 5). Ce paysage humanisé se caractérise par l'agrégation de roches décorées dans les secteurs de passage de certains des fleuves les plus importants, en signalant les zones de haute valeur économique. À partir du Paléolithique supérieur, la permanence de ces groupes trouve dans ces roches un argument indubitable de leur présence. Des symboles datables à partir $\mathrm{du} \mathrm{XI}^{\mathrm{e}}$ millénaire cal $\mathrm{BC}$ prennent place dans ces territoires et sur les mêmes panneaux ; ils assurent une continuité technique de grande valeur pour comprendre le développement des figurations postglaciaires. Ce développement très puissant permet de reconstruire des paysages culturels d'une grande ancienneté, comme ceux qui caractérisent certains secteurs du Douro, du Taje ou du Guadiana. Les signes et les couleurs authentifient l'histoire de ces pierres et les humanisent, rendant plus ostentatoire la réalité pour laquelle elles ont été créées par les groupes humains.

Dans la péninsule Ibérique, la possibilité d'associer les usages des pierres décorées au Paléolithique avec celles levées au Néolithique - comme preuves d'une matérialisation de la position des groupes humains qui les ont réalisées - existe. Les mêmes territoires sont signalés par des supports identiques, ou très proches, et les techniques utilisées reflètent des connaissances adoptées à partir de longues traditions. Cette hypothèse permet de relativiser l'idée selon laquelle le mégalithisme illustre l'implantation de l'économie néolithique dans les territoires jusque-là inoccupés. Au contraire, au moins à l'Ouest de la péninsule, le mégalithisme reflète une intensification démographique qui se lie sans difficulté avec les témoignages de peuplement que l'on peut déduire des sites du Paléolithique supérieur et du Mésolithique.

La réalité majeure des paysages construits (avec les menhirs qui donnent des références naturelles en pierre et les mégalithes qui reproduisent les espaces souterrains (Bueno et al. 2008, Calado 2002) commence à devenir perceptible dans les données archéologiques ibériques du $\mathrm{VI}^{\mathrm{e}}$ millénaire cal BC avec certains menhirs. Son extension la plus importante se place sans difficultés dès la fin $\mathrm{du} \mathrm{V}^{\mathrm{e}}$ millénaire et son apogée durant le $\mathrm{IV}^{\mathrm{e}}$ et le $\mathrm{III}^{\mathrm{e}}$ millénaire cal BC.

Mais les roches en place décorées et l'usage des grottes naturelles comme sépulture ne disparaissent pas ; cela continue à jouer un rôle dans le marquage des territoires occupés par les constructeurs de mégalithes. La péninsule Ibérique apporte des informations de grand intérêt pour l'intégration de toutes ces données et elle dispose de références symboliques qui permettent de connecter : les mégalithes, les menhirs, les roches et les abris décorés de plein air.

Ce système établi sur la longue durée de la Préhistoire européenne, consolide la capacité symbolique de ces groupes, leur intérêt pour signaler leurs propres territoires et révèle, très probablement, l'existence d'une mythologie autour d'usages et de délimitations de terres considérés comme spécifiques (Bueno et al. 2004). L'idée de considérer l'association de ces messages avec des éléments de caractère identitaire - comme une des manifestations de revendication territoriale la plus ancienne de l'Histoire - devient séduisante. La définition des caractéristiques des représentations humaines de la Préhistoire récente constitue donc un axe de travail intéressant qui apporte des résultats tangibles, spécialement en ce qui concerne les figurations sur les supports mégalithiques et sur les objets mobiliers (Bueno 1992, 2010 ; Bueno et Balbín 1994 ; Bueno et al. 2005 ; 
Hurtado 2008).

Durant plus de trois millénaires, des processus constants de transport et de fragmentation de grandes pierres reflètent une systématique symbolique d'une profonde signification idéologique. Une des données $\mathrm{du}$ plus grand intérêt, parmi celles récemment obtenues dans la péninsule Ibérique, est la constatation d'agrégations de menhirs en plein air qui subissent des séquences de regroupement, de réinstallation et de transformation dans des moments contemporains du développement des dolmens. Ceci laisse supposer que le comptage total des mobilisations de grandes pierres pourrait être évalué en relation avec les données démographiques traditionnellement admises dans les régions intérieures, comme l'Alentejo ou l'Andalousie.

Une autre approche à considérer, pour la compréhension de ces processus, réside dans la persistance d'inclure les pierres de "plein air» dans les sépulcres, comme une appropriation constante des ancêtres pour générer du passé. Le transport de menhirs et de stèles pour construire des mégalithes, leur fragmentation et leur adaptation aux espaces funéraires, suggèrent une démarche symbolique qui rappelle fortement celle reconnue de dépôts d'ossements humains dans les mégalithes ; fragmentés ou complets, ils sont déplacés dans les monuments jusqu'à leur dépôt définitif (Jones 2005 ; Bueno et al. 2013b, 2014 et a). Pierres et ancêtres suivent des processus très similaires de mouvement, de transformation et d'usage comme des référents du passé ; cette comparaison renforce l'hypothèse que certaines pierres matérialisent des images humaines.

Cette " genèse de passés » serait à la base de la construction de mégalithes, concédant aux chaînes opératoires de ces monuments un caractère idéologique remarquable dont témoigne la présence de stèles et de menhirs réutilisés. Ces mouvements de grandes pierres entre les monuments de plein air et les dolmens sont présents durant les $\mathrm{VI}^{\mathrm{e}}, \mathrm{V}^{\mathrm{e}}, \mathrm{IV}^{\mathrm{e}}$ et $\mathrm{III}^{\mathrm{e}}$ millénaires cal BC. La diachronie de ces processus est bien documentée dans la péninsule Ibérique et elle concerne sans problème tout le développement $\mathrm{du}$ mégalithisme. Plus précisément, cette large diachronie permet d'identifier les signalisations en pierre des sépulcres plus récents comme la survivance d'une longue association ancestrale entre pierres et images humaines (Bueno et al 2005 ; Bueno et al. 2011a, 2011b).

Si l'on accepte que les zones européennes qui présentent ces incorporations d'ancêtres dans les constructions mégalithiques soient considérées comme des centres originaires du phénomène mégalithique, il faut prendre en compte la péninsule Ibérique dans ces inventaires. Nonobstant, il est fort probable que des preuves de processus similaires seront mises en évidence si des travaux équivalents sont menés dans d'autres contextes du mégalithisme européen, utilisant des protocoles semblables aux nôtres. De même, une analyse détaillée du réemploi de stèles et de menhirs dans d'autres zones européennes permettra de valider les contemporanéités dans la séquence de construction des mégalithes.

L'hypothèse d'un langage sur le temps long, avec les pierres/ancêtres comme principaux acteurs, prend toute sa réalité dans les sites ibériques. Les roches décorées recueillent des images indélébiles du passé réalisées sur des supports pérennes qui ont perduré dans le temps ; elles ont enregistré l'information comme d'authentiques bibliothèques du passé.

Remerciements : Cet article est un des résultats du projet de recherche «Les couleurs de la mort » HAR2012-34709 financé et porté par le Ministerio de Economía y Competitividad espagnol. Nous sommes reconnaissants envers nos collègues J.A. Linares, et A. Vazquez Cuesta pour leur concours. La traduction du texte en espagnol a été faite par J.Calvo. 


\section{Bibliographie}

Aranda G., GARCía SANJuÁn L., LozAno A. et CoStA M.E. (2013). Nuevas dataciones radiométricas del dolmen de Viera (Antequera, Málaga). Menga, nº 4, p. 235-259.

BAlbín BERHMANN R. (2009). El Arte Rupestre Paleolítico al aire libre en la Península Ibérica. in Balbín R. (ed.) Arte Prehistórico al aire libre en el Sur de Europa. Salamanca, Ed. Junta de Castilla y León, p. 19-56.

Balbín Behrmann R. et Alcolea GonZalez J. (1999). Vie quotidienne et vie religieuse. Les sanctuaires dans l'art paléolithique. L'Anthropologie, 103, p. 23-49.

BAlbín BEHRMANN R. et Bueno RAMiRez P. (2009). Altamira, un siècle après: art paléolithique en plein air. L'Anthropologie, 113, p. 602-628.

Barroso R., Bueno P., Balbín R., VÁzquez A. et GonzÁlez A. (2012). Agregaciones protohistóricas a megalitos prehistóricos : El dolmen de Lagunita I, Santiago de Alcántara (Cáceres). Actas V Encontro Arqueología do Sudoeste Peninsular, Almodovar, Portugal, p. 99111.

BÉNÉTAu G. (2000). Les alignements de menhirs du Sud de la Vendée. Ed. Anthropologica, Toulouse.

BRADLEY R. (2000). An archaeology of natural places. Ed. Psychology Press, Routledge, London.

BRIARD J., GAUTIER M. et LEROUX G. (1995). Les mégalithes et les tumulus de Saint-Just (Illeet-Vilaine). Évolution et acculturations d'un ensemble funéraire (5000 à 1500 ans avant notre ère). Documents préhistoriques, 8, CTHS, Paris.

BUENO RAMíREZ P. (1992). Les plaques décorées alentejaines : approche de leur étude et analyse. L'Anthropologie, 96, p. 573-604.

- (1995). Megalitismo, estatuas y estelas en España. Statue-stele e massi incisi nell'Europ dell'etá del Rame. Notizie Archeologiche Bergomensi, n³, p.77-130.

- (2010). Ancestros e imágenes antropomorfas muebles en el ámbito del megalitismo occidental : las placas decoradas. In CACHO C., MAICAS R., GALÁN E. et MARTOS J.A. (coord.), Ojos que nunca se cierran. Ídolos en las primeras sociedades campesinas, Ed. Ministerio de Cultura, DVD.

- (2009). Espacios decorados al aire libre del occidente peninsular. Territorios tradicionales de cazadores-recolectores y de productores. in Balbín R. (ed), Arte Prehistórico al aire libre en el Sur de Europa, Junta de Castilla y León, p. 323-346.

Bueno RAMiRez P., BALBín BEHRMANN R. (1992). L'Art mégalithique dans la péninsule Ibérique. Une vue d'ensemble. L'Anthropologie, 96, p. 499-570.

- (1994). Estatuas-menhir y estelas antropomorfas en megalitos ibéricos. Una hipótesis de interpretación del espacio funerario. Homenaje a González Echegaray, Museo y Centro de Investigación de Altamira, Monografias, 17, p. 337-347.

- (2000). Art mégalithique et art en plein air. Approches de la définition du territoire pour les groupes producteurs de la péninsule Ibérique. L'Anthropologie, 103, p.427-458.

- (2002). L'art mégalithique péninsulaire et l'art mégalithique de la façade atlantique : un modèle de capillarité appliqué à l'art postpaléolithique ibérique. L'Anthropologie, 106, p. 603-646. 
- (2006). Arte megalítico en la Península Ibérica : contextos materiales y simbólicos para el arte esquemático. in MARTínEZ J. et HERNÁNDEZ M. (eds.), Arte rupestre esquemático en la Península Ibérica, Comarca de los Vélez, p. 57-84.

- (2009). Marcadores gráficos y territorios tradicionales en la Prehistoria de la Península Ibérica. Cuadernos de Prehistoria de la Universidad de Granada, 19, p. 65-100.

Bueno RAMiRez P., BAlbín Behrmann R. et AlColea GonZÁleZ J. (2003). Prehistoria del lenguaje. in BALBÍN R. et BUENO P. (eds.), El arte prehistórico desde los inicios del siglo XXI. Primer Congreso Internacional de Arte Prehistórico en Ribadesella, Ribadesella, p. 13-22.

- (2007a). Style V dans le bassin du Douro. Tradition et changement dans les graphies des chasseurs du Paléolithique supérieur européen. L'Anthropologie, 111, p. 549-589.

BuENO RAMIREZ P., BALBÍN BEHRMANN R. et BARROSO BERMEJO R. (2005). Hiérarchisation et métallurgie : statues armées dans la péninsule Ibérique. L'Anthropologie, 109, p. 577-640.

- (2004). Application d'une méthode d'analyse du territoire à partir de la situation des marqueurs graphiques à l'intérieur de la péninsule Ibérique: le Tage international. L'Anthropologie, 108, p. 653-710.

- (2007b). Chronologie de l'art mégalithique ibérique : C14 et contextes archéologiques. L'Anthropologie, 111, p. 590-654.

- (2008). Dioses y antepasados que salen de las piedras. Boletín del Instituto andaluz del Patrimonio Histórico, 67, p. 62-67.

- (2009b). Análisis de las grafías megalíticas de los dólmenes de Antequera y su entorno. in Dólmenes de Antequera: tutela y valorización hoy, Sevilla. Ed. Instituto Andaluz del Patrimonio Histórico (PH cuadernos 23), p. 186-197.

- (2010b). Grafías de los grupos productores y metalúrgicos en la cuenca interior del Tajo. La realidad del cambio simbólico. in Gonçalves V. et Sousa A.C. (eds), Transformaçao e Mudança no Centro e Sul de Portugal: o $4^{\circ}$ e o $3^{\circ}$ milénios a.n.e. Cámara Municipal de Cascais, p. 489-517.

- (2013b). Símbolos para los muertos, símbolos para los vivos. Arte megalítico en Andalucía. II Congreso de Arte esquemático, Los Vélez, Almería, p. 25-47.

- 2014, "Custodian stones: Human images in Megalithic buildings of the South of the Iberian Peninsula ", In A. CRUZ, E. CERRILlo-CuenCA, P.BuENO-RAMiREZ, J.CANINAS, C. BATATA eds: Rendering Death: Ideological and Archaeological Narratives from Recent Prehistory (Iberia). BAR S2648, Oxford, p. 3-12.

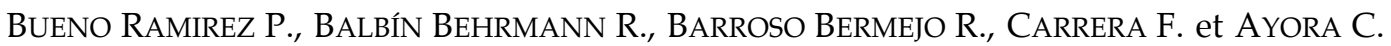
(2013c). Secuencias de arquitecturas y símbolos en el dolmen de Viera (Antequera, Málaga, España). Menga, 4, p. 251-266.

Bueno Ramirez P., Balbín Behrmann R., Barroso Bermejo R, Cerrillo Cuenca E., GONZÁlez CORDERO A. et PRADA A. (2011 ${ }^{\mathrm{a}}$ ). Megaliths and stellae in the inner basin of Tagus river. Santiago de Alcántara, Alconétar y Cañamero (Cáceres, Spain), in BuENO P. CERrillo E. et GONZÁleZ A. (eds.), From the origins. The prehistory of the inner Tagus Region, Oxford, Archaeopress (British Archaeological Report Series 2219), p. 143-158.

Bueno RAmirez P., BAlbín Behrmann R., BARRoso Bermejo R., LÓPEZ Quintana J.-C. et GUENAGA A. (2009a). Frontières et art mégalithique. Une perspective depuis le monde pyrénéen. L'Anthropologie, 113, p. 882-929. 
BuEnO RAmiREZ P., de BAlBín R., GutIÉRREZ J.-M. et EnRÍQueZ L. (2010c). Hitos visibles del megalitismo gaditano. Cuaternario y Arqueología: Homenaje a Francisco Giles Pacheco, Asociación Profesional de Patrimonio Histórico-Arqueológico de Cádiz y la Diputación Provincial de Cádiz, p. 209-228

Bueno RAmirez P., BAlbín Behrmann R., LAPORTE L., GOUEzin P., Coussea F., BARroso R., QUESNEL L., L 2015 « Natural colors / artificial colors. The case of Brittany's megaliths », Antiquity 89, p.55-71.

Bueno RAMirez P. BARRoso Bermejo R. \& BALbín BeHRMANn R. (2010a). Metal and the symbols of ancestors in Northern Iberia, in Proceedings of the XV UISPP World Congress (Lisbon, 4-9 September 2006), Vol. 41, Oxford, Archaeopress (British Archaeological Report Series 2058), p. 71-87.

- (2011b). Identidades y estelas en el Calcolítico peninsular. Memorias funerarias en la cuenca del Tajo, Sabugal. Libro de Actas IV Jornadas Raianas, p. 37-62.

- (2013a). Interior regions and places of collective memory: the Megalithism of the interior basin of the Tagus, Iberian Peninsula. A reflection after the reading of the Tara project. in O'Sullivan M., Scarre C. et Doyle M. (eds.), Tara - from the past to the future. Towards a new research agenda, Ed. Wordwell LTD and School of Archaeolgy, p. 484-502.

- (sous presse). Between East and West : megaliths in the Centre of the Iberian Peninsula. Oxbow, Oxford.

CALAdo M. (1997). Cromlechs alemtejanos e a arte megalítica, Brigantium, 10, p. 287-297.

- (2002). Standing stones and natural outcrops: the role of ritual monuments in the Neolithic transition of the Central Alentejo. in SCARRE Ch. (coord.) Monuments and landscape in Atlantic Europe. Perception and society during the Neolithic and early Bronze. London, Routledge, p. 17-35.

CARRERA RAMirez F. (2008). El dolmen de Os Muiños (Agolada, Pontevedra): intervención para la documentación y protección de la pintura megalítica conservada. Gallaecia, 27, p. 113-135.

- (2011). El arte parietal en monumentos megalíticos del Noroeste ibérico. Valoración, diagnosis y conservación. Oxford, Archaeopress (British Archaeological Reports International series 2190).

CARRERA F. et FÁBREGAS R. (2002). Datación radiocarbónica de pinturas megalíticas del Noroeste peninsular, Trabajos de Prehistoria, 59,1, p. 157-166.

CASSEN S. (2005). Pigeon-vole! Re-connaissance d'une gravure armoricaine du $\mathrm{V}^{\mathrm{e}}$ millénaire. Bulletin de la Société préhistorique française, 102, n² 2, p. 299-334.

D'ANNA A., MARChESI H., SOUlA F. et TRAMONI P. (2012). Les monuments mégalithiques de Cauria à l'âge du Bronze (Sartène). 20 années de recherche sur la préhistoire récente, p. 6163.

DARVILL T. (1997). Ever increasing circles : the sacred geographies of Stonehenge and its landscape. Proceedings of the British Academy, 92, p. 167-202

Foster M. (2013). An Animate Landscape: Rock Art and the Prehistory of Kilmartin, Argyll, Scotland. European Journal of Archaeology, 16, 4, p. 742-747. 
FURHOlT M., LÜTH F. \& MÜLLER J. (eds.) (2011). Megaliths and identities. Early monuments and Neolitihic Societies from the Atlantic to the Baltic. Bonn, Rudolf Habelt (Frühe Monumentalität und soziale Differenzierung, 1).

García SAnjuÁn L., Rivera T. et Wheatley D.W. (2003). Prospección de superficie y documentación gráfica en el dólmen del Llano de la Belleza, Aroche, Huelva. Anuario Arqueológico de Andalucía, p. 181-192.

GAVILÁN B. et VERA J.C. (2005). Neolítico y megalitismo prefunerario en Andalucía. III Congreso de Neolítico en la Península Ibérica, Santander, p. 535-541.

GOMES M.V. (1994). Menires e cromeleques no complexo cultural megalítico portuguêstrabalhos recentes e estado da questão. O megalitismo no Centro de Portugal, Mangualde Viseu, p. 317-342.

HENSEY R. \& Robin G. (2011). More than meets in the eye : new recordings of megalithic art in Nort-west Ireland. Oxford Journal of Archaeology, 30 (2), p. 109-130.

Herbault F. et Querré G. (2004). La parure néolithique en variscite dans le sud de l'Armorique. Bulletin de la Société préhistorique française, 101, 3, p. 497-520.

HuRTAdo V. (2008). Ídolos, estilos y territorios de los primeros campesinos en el sur peninsular. Acercándonos al pasado. Prehistoria en 4 Actos, Ministerio de Cultura, p. 1-11.

JONES A. (1999). Local Colour: Megalithic Architecture and colour Symbolism in Neolithic Britain. Oxford Journal of Archaeology, 18, p. 339-50.

JONES A. (2005). Lives in fragments? Personehood and the European Neolithic. Journal of Social Archaeology, 5, 2, p. 193-217.

JONES R. (2013). HRS Wales projects. Maerdy Windfarm, Glamorgan (2012-2013). http: / / www.hrswales.co.uk/ Projects.html

LAPORTE L. (2010). Restauration, reconstruction, appropriation; évolution des architectures mégalithiques dans l'Ouest de la France, entre passé et présent. Munibe, Suppl. XX, p. 15-46.

Le Roux C.T. (2006). Gavrinis et les mégalithes du golfe du Morbihan. Ed. Jean-Paul Gisserot, Paris.

L'HelgouACH J. (1983). Les idoles qu'on abat (ou les vicissitudes des grandes stèles de Locmariaquer). Bulletin de la Société polymatique du Morbihan, 110, p. 57-68.

LINARES J.A. (2011). Territorios, paisajes y arquitecturas megaliticas. Guía del megalitismo en la provincia de Huelva. Huelva, Ed. Junta de Andalucía.

López J. B., Moya A., Escala O. et Nieto A. 2009. La cista tumulària amb esteles esculpides dels Reguers de Seró (Artesa de Segre, Lleida) : una aportació insòlita dins l'art megalític peninsular i europeu. Tribuna d'Arqueologia, p. 87-125.

Loveday R. (2012). The Greater Stonehenge Cursus - the Long View. Proceedings of the Prehistoric Society, Vol. 78. p. 341-350.

Migdley M. S. (2013). Megaliths in North-west Europe. The Cosmology of Sacred Lanscapes. in TARLOW S. et NILSSON Stutz L. (dir.), The Archaeology of Death E Burial, Oxford Handboook, p. 421-440.

Moreno M. et Delibes G. (2007). Dataciones absolutas para un menhir del Valle de 
Valdelucios (Burgos) : resultados de un sondeo en el túmulo de la Cuesta del Molino. Zephyrus, 60, p. 173-179.

Moullec J-M., MARChand G., LÉOPOld P., Le Goffic M., KAYSER O. et GOUletQuer P-L. (1996). Où sont passés les Mésolithiques côtiers bretons? Bilan 1985-1995 des prospections de surface dans le Finistère. Revue archéologique de l'Ouest, 13, 1, p. 5-30.

O'Sullivan M. (2009). Living stones. Decoration and ritual in 4th and 3 th millennium BC. Ireland. in BALBíN R., BUENO P., GONZÁLEZ R. et del ARCO C. (eds.), Grabados rupestres de la fachada atlántica europea y africana, Oxford, Archaeopress (British Archaeological Report, International series, 2043), p. 5-12.

PEARSON M.P. \& RAMILISONINA (1998). Stonehenge for the ancestors : the stones pass on the message. Antiquity, vol. 72, n²76, p. 308-326.

RUST A. (1943). Die alt- und mittelsteinzeitlichen Funde von Stellmoor. Neumünster, Karl Wachlholtz.

Scarre C., Arias P., Burenhult G., FAno M., Oosterbeek L., Schulting R., Sheridan A. \& WhitTle A. (2003). Megalithic chronologies. in Burenhult G. (ed.), Stones and Bones; Formal Disposal of the Dead in Atlantic Europe during the Mesolithic-Neolithic Interface 60003000 BC, Oxford, Archaeopress (BAR International Series, 1201), p. 65-114.

SteElman K.L., RAmírez F.C., VAlCARCE R. F., Guilderson T. \& ROWE M.W. (2005). Direct radiocarbon dating of megalithic paints from north-west Iberia. Antiquity, 79, p. 379-389.

SHEE Twohig E. (1981). The megalithic art of Western Europe. Clarendon Press, Oxford. 


\section{Illustrations}

Figure 1. a : Bois décoré avec cercles concentriques de Maerdy, Wales, Royaume-Uni, (d'après Jones 2013) ; b: Gavrinis, Bretagne, France, (photo R. de Balbín); c: stèle mégalithique d'Oles, Asturies, Espagne, (d'après Bueno et al.2009).

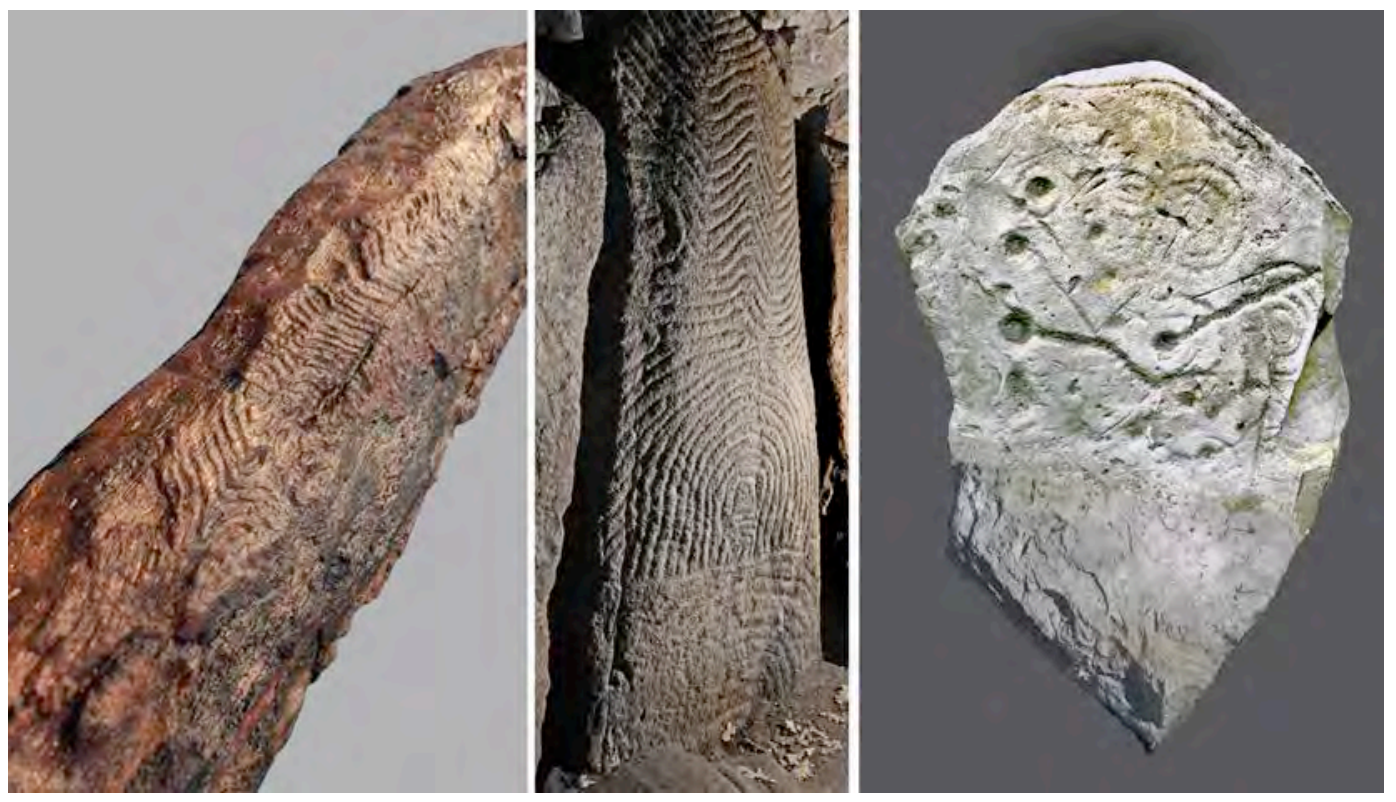

Figure 2. Menhir du Cabezo, Alcántara, Espagne. Vue générale du menhir tombé et du paysage de la plaine du Tage (Photo R. de Balbín).

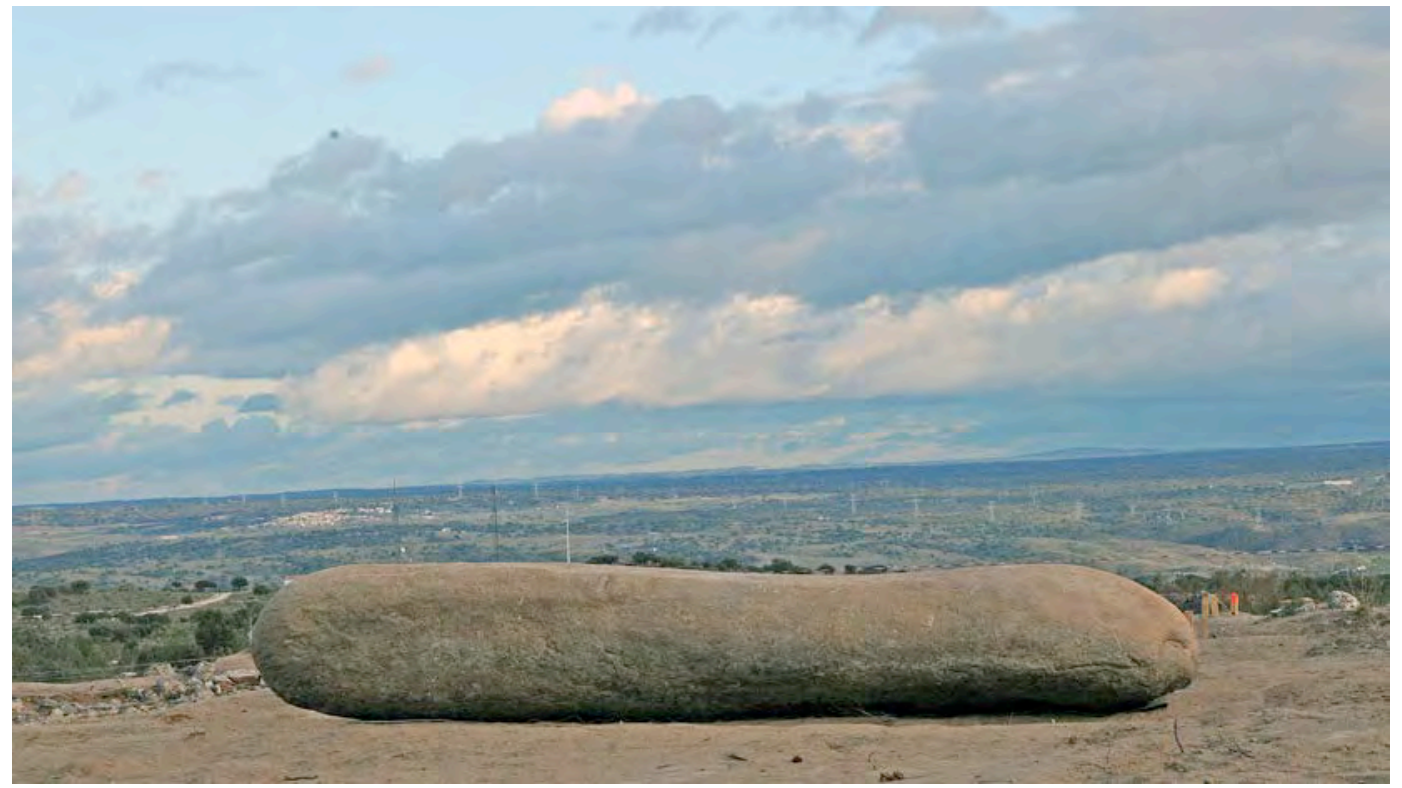


Figure 3. Stèle de Telhal du front de la chambre mégalithique de Telhal ( $\mathrm{d}$ 'après Bueno et al. 2013).

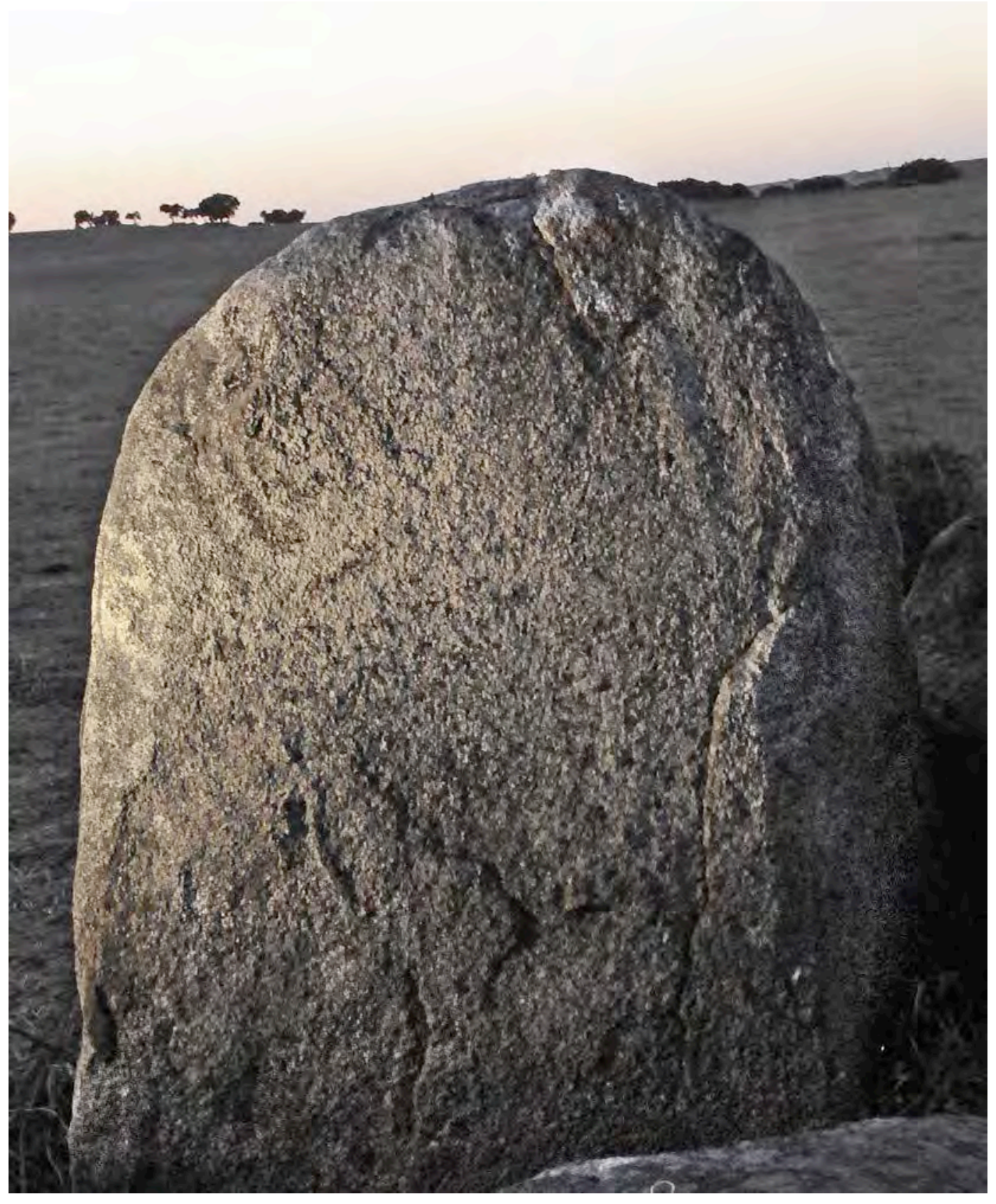


Figure 4. Élévation photographique des files Nord et Sud du dolmen de Soto, Huelva, Espagne (Photos R. de Balbín).

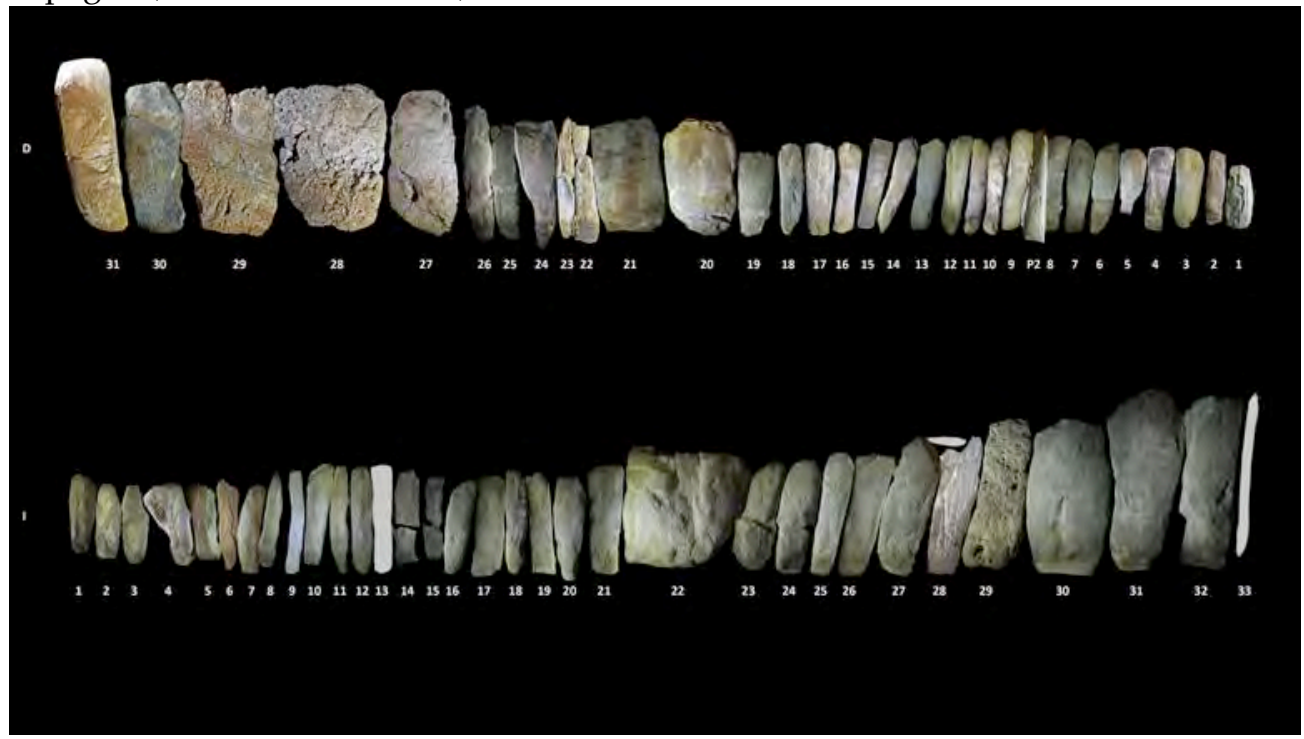

Figure 5. En haut: obscurité et fermeture au Paléolithique et au Néolithique. a. Grotte de Tito Bustillo, Asturies, Espagne ; b. dolmen de Petit Mont, Arzon, Bretagne.

En bas: verticalité et visibilité au Paléolithique et au Néolitique. c. Rocha dos Namorados, Vale do Côa Portugal; d. Menhir du Champ Dolent, Bretagne, France. (Photos R. de Balbín).
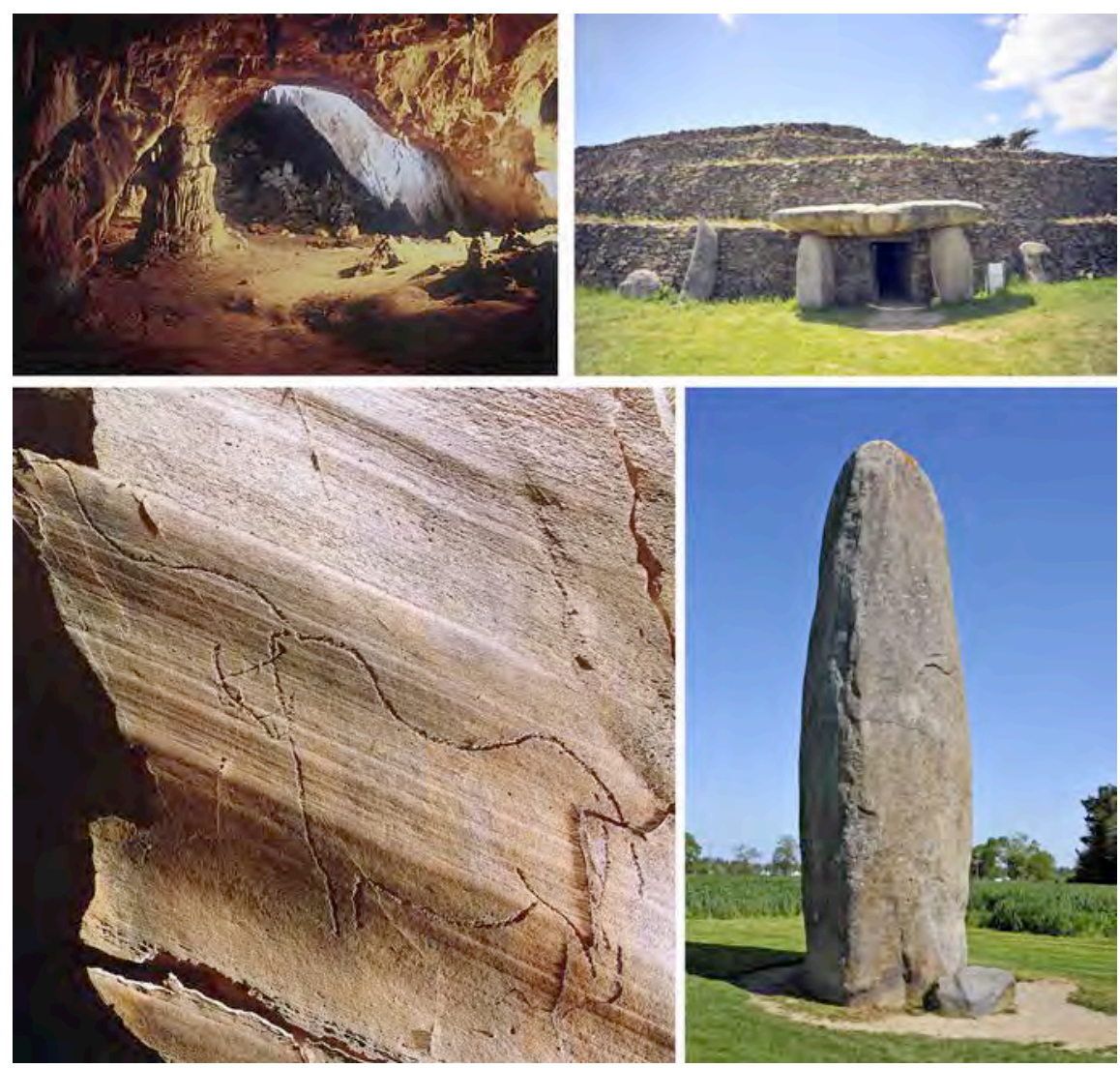


\title{
Pierres dressées et mâts-totem : le pilier comme vecteur de communication publique dans les sociétés pré-littéraires
}

\author{
Christian JEUNESSE \\ Université de Strasbourg, \\ UMR 7044 du CNRS, Institut Universitaire de France \\ Université de Strasbourg, MISHA \\ Extrait de : Olivier BUCHSENSCHUTZ, Christian JEUNESSE, Claude MORDANT et Denis VIALOU (dir.),
Signes et communication dans les civilisations de la parole, Paris,
Édition électronique du CTHS (Actes des congrès des sociétés historiques et scientifiques), 2016. \\ Cet article a été validé par le comité de lecture des Éditions du CTHS dans le cadre de la publication \\ des actes du $139^{\circ}$ Congrès national des sociétés historiques et scientifiques tenu à Nîmes en 2014.
}

\begin{abstract}
Résumé
Engagé dans une recherche de références ethnologiques dans le contexte d'une réflexion sur la signification du mégalithisme européen, nous avons été frappé par la diversité des circonstances dans lesquelles sont érigées des pierres dressées et la variété des messages dont elles constituent les supports. Dans certaines sociétés non-mégalithiques, à l'exemple des tribus indiennes de la côte Nord-Ouest de l'Amérique du Nord, les mêmes messages sont portés par des piliers en bois (les «mâts-totems»). D'autres sociétés, notamment en Afrique orientale, utilisent conjointement les deux types de supports. Dans des populations privées d'écriture, les piliers constituent, à côté de la communication orale, un support privilégié de transmission de la mémoire collective. Par leur biais, statuts (des vivants ou des morts), généalogies, épisodes mythologiques, événements - anodins ou marquants - de la vie de la communauté forment un paysage mémoriel beaucoup plus coloré et divers que ne le laissent supposer l'austérité apparente de nos mégalithismes fossiles européens.
\end{abstract}

Mots-clés : mât-totem, menhir, pierres dressées, statue-menhir, mégalithisme

\begin{abstract}
Whilst engaging in research on the significance of European megalithism using ethnological references, we were struck by the diversity of the circumstances in which these stones were erected and by the messages they convey. In non-megalithic societies, for example the native tribes of the North-West coast of the United States, the same messages are conveyed using wooden totem poles. Other societies, in particular in East Africa, use both media. In oral societies, these pillars (and oral communication) which transcribe the collective memory: status (of the living and the dead), genealogies, myths, important or anodyne events of community life, make up a more colourful and more diverse memorial landscape than the supposedly more austere environment conveyed by European stone megaliths. (traduction Rebecca Peake)
\end{abstract}


Nous entendons par «pierre dressée» tous les types de pierres dressées isolées dépourvues de fonction architectonique, décorées ou non. La catégorie, qui recouvre au fond ce que la tradition nomme "menhir », englobe donc les statues-menhirs, les pierres isolées et les pierres intégrées dans une structure de type alignement ou enceinte. Leur usage est largement répandu et partagé par plusieurs provinces mégalithiques préhistoriques et subactuelles, comme l'a naguère bien montré R. Joussaume (1985). Dans les études consacrées au mégalithisme néolithique européen, la discussion tourne généralement autour d'une gamme d'interprétations relativement étroite s'appuyant, traditionnellement, sur deux grands paradigmes: les pierres dressées comme stèles funéraires ou comme élément d'un alignement ou d'un observatoire à vocation astronomique. Le tout avec, au fond, fort peu de certitudes. Les liens avec le funéraire sont loin d'être aussi directs qu'on le laisse parfois entendre. Ainsi à Sion "PetitChasseur », toutes les stèles ont été trouvées en position secondaire, réemployées dans l'architecture de sépultures à coffres de pierres; nous ne savons donc rien ni sur leur fonction originelle, ni sur leur lien chronologique avec les structures funéraires (parmi l'abondante bibliographie, voir en dernier lieu Corboud et Curdy 2009). Il en est de même pour les stèles aniconiques de la nécropole de Ventabren (Bouches-du-Rhône, Néolithique récent et final) (Hasler 1998 ; Hasler et al. 2002), elles aussi probablement réutilisées après une première "vie " que rien n'oblige à mettre en relation avec un contexte funéraire, et aussi, enfin, pour la célèbre série de Gavrinis, formée elle aussi de stèles réemployées (voir en dernier lieu Cassen et al. 2014) dont la fonction primaire demeure tout aussi énigmatique. Le cas des stèles du Néolithique final I d'Italie du Nord présente ceci d'intéressant qu'on en a trouvé récemment au sein de structures interprétées comme des sanctuaires (en dernier lieu Fedele 2013), sans connexion directe avec des installations funéraires. L'interprétation " astronomique » n'est, pour sa part, véritablement convaincante que pour un petit nombre de monuments, par exemple le grand sanctuaire de Stonehenge (dont les supports verticaux des célèbres tritlithons n'entrent d'ailleurs pas dans notre définition des pierres dressées).

On trouve dans la littérature sur le mégalithisme européen d'autres interprétations plus générales : les pierres dressées comme représentations phalliques, ou encore l'idée d'une verticalité qui favoriserait la communication entre le monde sensible et le monde surnaturel. Certains ont également insisté sur l'efficacité médiatique du support « pilier », qui n'a pas échappé aux publicistes et aux propagandistes de toutes obédiences. Il s'agit là de généralités certes incontournables, mais sans grande utilité concrète. D'autres modèles plus ambitieux, certains ne s'appuyant que très marginalement sur les comparaisons ethnographiques (notamment: D'Anna et al. 2006 ; Cassen 2014 a et b), d'autres davantage (par exemple : Parker Pearson et Ramilisonina 1998 ou Cauwe 2014) ont été développés. Ils ne seront cependant pas discutés dans cet article dans lequel nous n'avons aucunement la prétention d'apporter une contribution directe à la question de l'interprétation du mégalithisme néolithique européen.

Notre parti pris est ici, indépendamment de toute référence aux pierres dressées européennes et aux discussions qu'ont suscitées leur compréhension, de dresser un inventaire préliminaire des significations recueillies en contexte ethnologique. L'objectif n'est pas, comme dans le comparatisme "sauvage ", de trouver des clés d'interprétations toutes faites qu'on pourrait transposer dans le mégalithisme néolithique européen, mais simplement, comme dans toute entreprise de ce type, d'élargir l'éventail des hypothèses auxquelles confronter les données recueillies sur les mégalithismes "fossiles ». Nous nous sommes donc livré, en marge d'un travail plus large sur les sociétés mégalithiques subactuelles, à une petite enquête ethnographique.

Cette recherche n'a aucune prétention à l'exhaustivité. Il faut la voir plutôt comme une série de sondages destinés à réaliser un inventaire préliminaire des fonctions observées dans les sociétés actuelles et subactuelles. Nous nous sommes intéressé à des sociétés qui ont pu être observées "vivantes », dans lesquelles voyageurs, missionnaires, soldats ou savants ont pu recueillir auprès d'informateurs autochtones des indications sur la 
signification et les contextes d'érection des pierres dressées. Les synthèses produites par R. Joussaume (Joussaume 1985 ; compléments dans Joussaume et Raharijaona 1985) et A. Gallay (2006) ont servi de point de départ à cette recherche. Pour ce qui est de l'Asie du Sud-Est, grand réservoir de données s'il en est, nous avons complété notre information en puisant dans les monographies de T.C. Hodson sur les Naga (1911), de E.E.W. Schröder sur les Nias (1917) et de C. Jannel et F. Lontcho sur les Toraja (1992), ainsi que dans deux articles de T. Sudarmadi (1999) et A.-M. Viaro (1984).

Sans que cela soit une véritable surprise, nous avons été frappé par la très grande diversité des usages, que ce soit pour les circonstances dans lesquelles sont érigées des pierres dressées ou pour la variété des messages dont elles constituent les supports. Nous allons voir que cela nous mène bien au-delà de la fonction funéraire et des observatoires, et que la pierre dressée peut être vue, de manière générale, comme un des supports de mémoire utilisés par les sociétés dépourvues d'écriture.

\section{Les données}

Les données recueillies proviennent d'une aire très vaste qui va de l'Afrique orientale à l'île de Pâques (fig. 1). Il existe bien d'autres concentrations de mégalithes de par le monde, mais elles correspondent à des mégalithismes fossiles, connus uniquement par le biais des recherches archéologiques. Commençons par passer en revue brièvement les différents contextes culturels pris en compte.

Les Nagas de l'Assam, dans le Nord-Est de l'Inde, érigent des menhirs isolés ou groupées dans des alignements ou des structures circulaires délimitant des aires de danse utilisées notamment dans le cadre du culte des ancêtres (Hodson 1911). Certains menhirs sont de fait érigés en l'honneur des ancêtres ou de certains défunts, d'autres sont dressés dans le cadre de fêtes de prise de grade (ou «fêtes du mérite »). Ces dernières sont réservées à l'aristocratie locale; l'érection des pierres constitue un des points culminants de ces cérémonies fastueuses exigeant des moyens très importants. Dans une des zones concernées, l'échelle des grades compte six niveaux; chaque promotion suppose, de la part du postulant, l'organisation d'un banquet. Deux pierres - une pour lui, une pour sa femme - sont dressées devant sa maison lors de la fête d'accès au sixième grade (fig. 2). Traditionnellement, l'accès à certains grades supposait également l'exhibition d'une tête d'ennemi fraîchement coupée (Gallay 1995). À côté des pierres liées à ces cérémoniels ostentatoires individuels et aristocratiques ou utilisées dans le cadre des funérailles ou du culte des ancêtres, d'autres, dans une démarche plus collective, sont dressées pour commémorer des événements mythiques.

L'île de Flores, en Indonésie, où existent par ailleurs des coffres funéraires mégalithiques, a livré au moins deux types de pierres dressées. Certaines, groupées en «fagots » et érigées à l'occasion de la mort d'un guerrier, représentent les ennemis qu'il a tués au combat, s'apparentant ainsi à des sortes de trophées symboliques. D'autres ont une signification religieuse, comme ces menhirs érigés aux deux extrémités des villages et associés à un culte solaire. Les pierres-trophées illustrent bien la diversité des significations: une pierre peut représenter le guerrier lui-même, d'autres les ennemis qu'il a occis, le dénominateur commun étant qu'elles célèbrent toutes deux la gloire de l'intéressé.

Sur l'île de Nias, au large de Sumatra, on trouve un mégalithisme polymorphe, avec des sièges, des dalles horizontales servant de litières cérémonielles et des pierres dressées (Schröder 1917, Viaro 1984). Souvent sculptées, ces dernières sont érigées sur les places des villages, devant les maisons des nobles, soit à l'occasion de fêtes de prise de grade, soit lors de funérailles; dans le second cas, les pierres servent de siège à l'esprit du mort. Certaines stèles anthropomorphes anciennes sont réputées être en relation avec le culte des ancêtres. 
Chez les Toraja de Sulawesi, toujours en Indonésie, le mégalithisme est essentiellement lié aux cérémonies funéraires. Lors des funérailles des membres de la noblesse, des menhirs sont dressés dans une aire cérémonielle. Ils ne sont pas directement associés à la sépulture, qui est installée dans une cavité creusée à flanc de falaise. L'érection du menhir fait partie d'un cycle cérémoniel complexe avec un grand déploiement de richesse et de puissance. Ce cycle est rythmé par les rites favorisant la libération des âmes du défunt : la première s'en va dans le premier bœuf sacrifié, la seconde dans l'effigie de bois du défunt qui sera exposée devant le caveau familial, la troisième dans ce menhir que l'on dresse dans l'aire sacrée juste avant que le corps ne soit conduit dans la tombe, dans le cadre d'une cérémonie impliquant des sacrifices de buffles et de cochons (Gallay 2011, Jannel et Lontcho 1992).

À Madagascar, dans les ethnies Merina et Betsileo héritières des migrants austronésiens venus d'Indonésie, on trouve, parallèlement à des coffres funéraires mégalithiques, de nombreuses pierres dressées aux significations variées. Certaines sont érigées sur des plates-formes à caractère funéraire, où elles côtoient des piliers sculptés en bois et représentent un ancêtre fameux. D'autres, chez les Mérinas, se dressent auprès de la tombe du lignage, servant de substitut à un ancêtre décédé au loin dont le corps n'a pas pu être déposé dans la sépulture collective ; il est à noter que ces substituts sont tantôt en pierre, tantôt en bois. Dans la province d'Imerina, la limite des villages traditionnels est marquée par quatre mégalithes dressés aux quatre coins cardinaux; on est là, comme à Flores, du côté de l'astronomique et du religieux, qui ne sont évidemment pas séparables. Mais il existe bien d'autres prétextes à l'érection de menhirs : commémoration d'une alliance politique, d'une déclaration de guerre ou d'une réconciliation, intronisation d'un prince, ou encore construction d'une résidence royale. L'érection des pierres s'inscrit donc, de manière courante, dans les cérémonies accompagnant les grands événements de la vie communautaire.

En Polynésie, les pierres dressées sont en général liées aux fameuses plateformes cérémonielles appelées ahu sur l'île de Pâques, heiau à Hawaï et marae à Tahiti, dans les Tuamotou (fig. 3) ou à Samoa. Les statues anthropomorphes pascuanes (moai) sont érigées à la mémoire de personnages de haut rang ou représentent les ancêtres des lignages. L'ahu est un monument plurifonctionnel, mêlant les dimensions religieuse, funéraire et identitaire. À Tahiti et Samoa, on trouve à la fois des menhirs érigés sur la plateforme et des dalles dressées insérées dans son dispositif de délimitation.

L'Éthiopie nous livre également un gros corpus partagé entre des mégalithismes soit complètement fossiles, soit fossiles mais encore vivants dans les mémoires, soit actuels. Chez les Arsi, on trouve des stèles à motifs géométriques directement associés aux tombes. Leur finalité est de célébrer la bravoure et la virilité du défunt, les conditions pour en être doté étant $\mathrm{d}^{\prime}$ avoir tué un ennemi, $\mathrm{d}$ 'avoir chassé avec succès un éléphant, un lion et un léopard et, enfin, d'avoir engendré des enfants, le nombre de ceux-ci déterminant le nombre de stèles. Chez les Konso, les pierres dressées se trouvent principalement dans deux contextes : premièrement des pierres couplées avec des tombes de grands guerriers se présentant sous la forme de grandes plateformes; elles y sont associées à des statues de bois représentant le guerrier, ses femmes et, comme sur l'île de Flores, les ennemis qu'il a tués. Deuxièmement, des pierres dressées sur une place du village soit à l'occasion de cérémonie de passage d'une classe d'âge à une autre, soit pour commémorer des événements importants, par exemple des conflits.

Là comme ailleurs, on remarque que les mêmes fonctions peuvent être remplies indifféremment par des piliers en pierre ou en bois. Cette substituabilité nous suggère que les sociétés non mégalithiques à piliers de bois doivent être associées à la réflexion globale sur la question des pierres dressées. Dans certaines tribus indiennes de la côte nord-ouest de l'Amérique du Nord, par exemple, les fonctions assumées ailleurs par des menhirs le sont par des piliers en bois souvent appelés de manière réductrice mâtstotems. Ces derniers peuvent être des «mâts-tombeaux» qui abritent physiquement les 
os du défunt déposés dans un coffre posé au sommet du pilier érigé devant la maison du lignage. D'autres piliers sculptés plantés eux aussi devant la maison peuvent raconter l'histoire, mélange hagiographique de faits réels et mythiques, du lignage, et d'autres être dressés en l'honneur d'invités prestigieux. D'autres encore, dans un tout autre registre, sont érigés pour faire honte à une personne qui s'est abstenue de payer une dette. Le monument est alors appelé shame pole; il signe l'un des rares cas où l'érection de piliers s'éloigne clairement de la dimension commémorative. Il y a donc une sorte de continuité entre les sociétés qui, pour exprimer une gamme de messages qui se recoupent largement, n'utilisent que la pierre, celles qui font appel au bois et à la pierre et celles qui, à l'image des tribus de la côte nord-ouest, n'emploient que le bois. On peut noter au passage que, si elle a bien sûr disparu des paysages, la mixité pierre-bois est bien attestée pour le mégalithisme néolithique européen. On sait aussi, depuis peu, qu'une partie au moins des pierres dressées du même complexe mégalithique portaient des décorations peintes (Bueno Ramirez et al. 2009, Bueno Ramirez et al. 2012). Mais l'ethnographie nous livre également des menhirs à décoration amovible, comme cette étonnante pierre dressée à vocation funéraire Gewada photographiée par J.-P. Cros et R. Joussaume il y a quelques années en Éthiopie et dont le caractère anthropomorphe est renforcé par la présence de véritables vêtements (fig. 4).

À l'image des menhirs indonésiens abritant l'esprit d'un ancêtre, les pierres dressées sont donc, au moins dans certains cas, autre chose que de pesants monolithes commémorant des événements passés; ils s'inscrivent dans le paysage comme des éléments vivants et agissants que l'on côtoie avec crainte et respect.

\section{Discussion}

Les données exposées laissent apparaître des contextes d'érections relativement variés et une large gamme de messages. En voici une rapide récapitulation :

- en contexte funéraire, le pilier peut représenter le mort lui-même, ses proches ou témoigner de sa bravoure et de sa virilité à travers la représentation ou l'évocation des ennemis ou des animaux dangereux qu'il a tués et des enfants qu'il a engendrés; il peut également abriter les restes du mort ou, plus souvent, offrir un abri à son esprit, et donc servir de demeure à un ancêtre dont la présence en quelque sorte physique renforce le poids dans la vie du groupe ;

- la représentation ostentatoire des hauts faits d'un individu se retrouve dans les pierres érigées, en Indonésie et en Assam, à l'occasion des cérémonies de prise de grade. Il s'agit de proclamer à la face du monde le prestige et la puissance d'un individu cette fois vivant. Le contexte est celui des ploutocraties ostentatoires d'A. Testart, où c'est, avant la bravoure au combat ou les prouesses cynégétiques, la richesse qui ouvre la voie à l'ascension dans l'échelle du prestige (Testart 2004);

- d'autres pierres commémorent des événements, réels ou mythiques : elles marquent le lieu d'un épisode mythologique ou rappellent le souvenir d'une bataille, d'une déclaration de guerre, d'un traité de paix, de la conclusion d'une alliance, de la construction d'un édifice, d'une naissance aristocratique, d'une cérémonie d'initiation, de l'intronisation d'un dirigeant, etc. ;

- quand ils sont décorés, les piliers, en pierre ou en bois, peuvent aussi raconter les hauts faits d'un lignage, ou servir à humilier un débiteur ;

- des pierres dressées sont également partie prenante de dispositifs religieux; ainsi ces blocs érigés aux confins des villages selon les points cardinaux, à Florès et à Madagascar ; - l'érection des menhirs peut s'accompagner de sacrifices animaux, comme dans les cérémonies funéraires Toraja; certains servent de support à des rituels divers dont l'étude systématique reste à faire ; celles qui abritent les esprits des ancêtres peuvent être vues comme des éléments vivants et agissants du paysage familier ;

- les pierres dressées et les piliers de bois sont toujours des monuments publics, même si certains célèbrent des mérites individuels. 
Ils peuvent être vus comme des supports de communication non-verbaux, avec, à première vue deux sortes de messages :

- stèles funéraires et pierres de prise de grade servent d'instruments de glorification pour les élites dans le domaine austronésien (Madagascar, Indonésie, Mélanésie et Océanie) et en Assam ;

- dans l'ensemble de notre domaine d'étude, d'autres piliers, à vocation commémorative, servent de support à la mémoire tribale.

Mais cette opposition n'est pas aussi tranchée qu'il n'y paraît. En effet, ce que célèbrent les pierres de mémoire, ce sont les victoires de l'aristocratie, la naissance des princes, la construction de la résidence nobiliaire, tout comme les âmes dont l'érection du menhir funéraire toraja contribue à la libération sont les âmes des riches et les "mâts-totem » historiés de la côte Nord-Ouest racontent l'histoire, réelle mais aussi mythique, des lignages nobles, soulignant la relation particulière au monde des dieux et des esprits qui fonde leur puissance. C'est donc, de manière générale, la geste des puissants qui est comme inscrite dans le paysage grâce aux piliers de bois ou de pierre. Ces derniers se font en quelque sorte écho: la victoire commémorée par un menhir sera celle d'un ancêtre dont l'esprit réside dans un autre menhir érigé non loin de là. Ce personnage, et à travers lui le lignage auquel il appartient, sera donc doublement présent dans le paysage mémoriel dont les pierres dressées forment autant de jalons. Leur érection sert à proclamer le prestige des puissants, mais aussi, du fait de leur solidité et de la résistance des matériaux employés, à prolonger dans le temps le retentissement de l'événement qui fut le prétexte de leur érection et la gloire du commanditaire. À l'exaltation d'une fête destinée à marquer durablement les esprits, succède la fréquentation quotidienne d'un pilier commémoratif qui rappelle à la fois l'événement lui-même et les rapports sociaux (relations hiérarchiques) qu'il a permis de revivifier et de consolider.

Nul autre événement ne témoigne de manière aussi éloquente de l'ambiance de compétition sociale exacerbée qui règne dans les sociétés concernées, au sein de systèmes où la valeur d'un individu se mesure à sa place dans l'échelle du prestige, où sa position dans la hiérarchie n'est pas garantie par des statuts politiques institutionnalisés et doit donc être constamment rappelée et raffermie par l'organisation de nouvelles fêtes et l'érection de nouveaux piliers. Il existe donc, manifestement, un lien entre les cultures à pierres dressées et certaines configurations socio-politiques. Un gouverneur de province d'un État centralisé n'a évidemment pas besoin de dresser une pierre tous les ans pour afficher sa position dans l'ordre de préséance, puisque son statut est inscrit dans le marbre de l'organisation étatique. Le pilier sert à conserver la mémoire du lignage et offre une version indurée, éventuellement historiée, des récits qui sont la matière du discours des griots ou des aèdes lorsqu'ils énumèrent, à l'occasion des fêtes, les hauts faits et la généalogie des puissants. Il témoigne d'une volonté pathétique de projeter vers le futur l'état des rapports de force qui prévaut au moment de son érection.

De quel futur parlons-nous ? Quelle est la durée de cette mémoire des pierres et des piliers et, accessoirement, qui est chargée de la conserver? Nous n'avons pas trouvé de travaux sur ce sujet, pour lequel certains villages indonésiens où cohabitent pierres « vivantes » et «fossiles » constitueraient des terrains idéaux. On peut cependant avancer sans trop de risque que, dans cette ambiance où le rang social doit être sans cesse réaffirmé, l'effacement de la mémoire des pierres dressées qui renvoient aux mérites individuels ou à l'histoire du lignage doit suivre de peu la disparition (ou la dégradation) de ce dernier. Dans le cadre de cette hypothèse, il faut envisager que les paysages mégalithiques et les configurations mémorielles qu'ils dessinent se renouvellent en permanence, montrant à un moment donné un mélange de pierres « mortes », celles des individus qui n'ont plus de descendants pour entretenir leur mémoire, et de pierres "vivantes » témoignant de l'état présent des rapports sociaux au sein de la communauté. Les pierres dressées à l'occasion d'une victoire collective, celles qui commémorent un événement mythique ou celles qui se dressent aux entrées des villages demeurent très probablement " actives » beaucoup plus longtemps. Leur destin à toutes est, sur la longue durée, au rythme des changements civilisationnels, de se voir investies de contenus 
nouveaux sans rapports avec les motivations qui furent celles de leurs commanditaires. Toute la force du médium "pilier» est dans cette capacité à dialoguer avec des imaginaires successifs.

Cette manière de fixer la mémoire et de rendre tangible l'ordre social en dressant des piliers a probablement été beaucoup plus répandue que nous ne le laisse entrevoir la répartition chronologique et géographique des grandes provinces mégalithiques. Les sociétés anciennes qui, à l'image des Indiens de la Côte Nord-Ouest, ont choisi de « communiquer » exclusivement à travers des piliers de bois resteront forcément à l'écart de nos inventaires. Un examen superficiel des données ethnologiques disponibles montre cependant que ce choix est loin d'être aussi répandu qu'on pourrait le penser. Il s'agit donc bien d'un mode de communication spécifique, historiquement et culturellement situé, et qui mériterait de ce fait que l'on s'y intéresse davantage afin de mieux en saisir la signification et les modalités de fonctionnement. La place des pierres dressées au sein des sociétés dites "mégalithiques» vivantes mériterait également un surcroît d'investigations. Au sein du corpus examiné, le spectre va des groupes - par exemple les Arsi et les Konso d'Éthiopie - pour lesquels le pilier est la seule manifestation mégalithique - à ceux où il ne constitue qu'un type de construction parmi d'autres. Le lien entre les seconds et les populations de langues austronésiennes disséminées entre l'île de Pâques et Madagascar (fig. 1), naguère suggéré par H. Geldern, paraît indiscutable (Geldern 1945). Une analyse détaillée des modalités de propagation et de la chronologie de cette tradition mégalithique spécifique pourrait être d'un grand secours pour la compréhension du mégalithisme néolithique européen. 


\section{Bibliographie}

BuenO RAMíRez P., BALbín BEHRMANN R. de, BARROSO BERMEjO R. (2009). Pintura megalítica en Andalucía. in CRUZ-Auñón BRIONES R. et FERRER AlBELDA E., dir., Estudios de Prehistoria y Arqueología en homenaje a Pilar Acosta Martínez, Universidad de Sevilla, Sevilla, p. 141-170.

Bueno Ramírez P., BAlbín Behrmann R. de, BARRoso Bermejo R., LAPORTE L., Gouezin P., BARRoso Bermejo R., HeRnANZ Gismero A., GAVIRA-VAllejo J.M. \& IRIARTE CELA M. (2012). Paintings in Atlantic Megalithic Art: Barnenez. Trabajos de Prehistoria 69, $\mathrm{n}^{\circ}$ 1, enero-junio 2, p. 123-132.

CASSEN S. (2014). Sites de Passage (1), Le modèle carnacois des pierres dressées à l'épreuve des rivières, des lacs et des montagnes (France, Suisse, Italie). in Entre archéologie et écologie, une préhistoire de tous les milieux, Mélanges offerts à Pierre PÉTREQUIN, Annales littéraires de l'Université de France Comté, Presses universitaires de Franche Comté, p. 281-302.

CASSEN S. (2014) Sites de passage 2, Le modèle carnacois des pierres dressées à l'épreuve des steppes, des courses et des légendes. in ROBIN G. et al., (dir.), Fonctions, utilisations et représentations de l'espace dans les sépultures monumentales du Néolithique européen. Actes du colloque international, Aix-en-Provence, 8-10 juin 2011, Préhistoires Méditerranéennes, supplément $n^{\circ} 3$.

Cassen S., Grimaud V., Lescop L., Marcoux N., Oberlin C. et Guirec Q. (2014). The first radiocarbon dates for the construction and use of the interior of the monument at Gavrinis (Larmor-Baden, France). PAST 77, July 2014, p. 1-4.

CAuwe N. (2014). Megalith of Ister Island. In Besse M. (dir.), Around the Petit-Chasseur Site in Sion (Valais, Switzerland) and New Approaches to the Bell Beaker Culture. Proceedings of the International Conference held at Sion (Switzerland) October 27th - 30th 2011, Oxford, Archeopress, p. 321-330

CORboud P. et CuRdy P. (dir.) (2009). Stèles préhistoriques. La nécropole du Petit-Chasseur à Sion. Musées cantonaux du Valais, Sion, 119 p.

D'Anna A., Guendon J.-L., Pinet L., Tramoni P. (2006). Espaces, territoires et mégalithes: le Plateau de Cauria (Sartène, Corse du Sud) au Néolithique et à l'Âge du Bronze. in DUHAMEL P. (dir.), Impacts interculturels au Néolithique moyen, Actes du $25^{\text {ème }}$ colloque interrégional sur le Néolithique, Dijon, octobre 2001, Revue archéologique de l’Est, $25^{\text {ème }}$ supplément, p. 191-213.

FEDELE F. (2013). Il sito cerimoniale di Anvòia a Ossimo (Valcamonica). in MARINIS R.C. de (dir.), L'Età del Rame. La Pianura Padana e le Alpi al tempo di Ötzi, Catalogue d'exposition, Massetti Rodella Editori, Roccafranca, p. 197-207.

FLANNERY K. \& MARCUS J. (2012). The creation of inequality : how our prehistoric ancestors set the stage for slavery, monarchy and empire. Harvard University Press, Cambridge \& London, 648 p.

GALLAY A. (1995). Mégalithisme et chefferie: approche transculturelle. in GALLAY A. (dir.), Dans les Alpes à l'aube du métal. Archéologie et bande dessinée, Sion, Catalogue édité par les Musées cantonaux du Valais, p. 163-171.

GALLAY A. (2011). Une approche anthropologique de la notion de bien de prestige. Bulletin d'études préhistoriques et archéologiques alpines, vol. XXI, Société valdôtaine de Préhistoire et d'Archéologie, p. 29-43. 
Gallay A. (2006). Les sociétés mégalithiques. Pouvoir des hommes, mémoire des morts. Lausanne, Presses polytechniques et universitaires romandes, Coll. "Le Savoir Suisse », $139 \mathrm{p}$.

HASLER A. (1998). Les stèles de la nécropole tumulaire néolithique de Château-Blanc (Ventabren, Bouches-du-Rhône), Archéologie en Languedoc 22, p. 105-112.

Hasler A., Collet H., Durand C., Chevillot P., Renault S. et Richier A. (2002). Ventabren - Château Blanc. Une nécropole tumulaire néolithique. in GUTHERZ X. (éd.), Archéologie du TGV Méditerranée. Fiches de synthèse, tome 1 : La Préhistoire, p. 227-238.

HEINE GELDERN R. (1945). Prehistoric Research in the Netherland Indies. in P. HONIG P. \& VERDOORN F. (dir.), Science and Scientists in the Netherlands Indies (New York: Board for the Netherlands Indies, Surinam and Curaçao, 1945). Reprint: (New York: Southeast Asia Institute, 1945), p. $129-167$.

Hodson T. C. (1911). The Naga tribes of Manipur. London, Macmillan \& co., 212 p.

Jannel C. et Lontcho F. (1992). Les Toraja d'Indonésie. Paris, Armand Colin, 161 p.

JOUSSAUME R. (1985). Des dolmens pour les morts. Les mégalithismes à travers le monde. Paris, Hachette, 398 p.

JOUSSAUME R. et RAHARIJAONA V. (1985). Sépultures mégalithiques à Madagascar. Bulletin de la Société préhistorique française, vol. 82, p. 431-451.

PARKer PEARSON M., RAMilisonina 1998, Stonehenge for the ancestors : the stones pass on message, Antiquity, t. 72, n²76, p. 308-326

RENFREW C. (1984). Islands out of time. in RENFREW C. (dir.), Approaches to social archaeology, Edinburgh University Press, p. 200-224.

SCHRÖDER E.E.W. (1917). Nias : ethnographische en historische aanteekeningen en studiën. Leiden, $866 \mathrm{p}$.

SUDARMADI T. (1999). A semiotic approach on indonesian megalithihs study, Humaniora, vol. 12, p. 73-81.

TeSTART A. (2005). Eléments de classification des sociétés. Ed. Errance, Paris, 159 p.

ViARO A. M. (1984). Nias : habitat et mégalithisme. Archipel, vol. 27, p. 109-148. 


\section{Illustrations}

Figure 1: Localisations des mégalithismes subactuels et frontières de l'aire des langues austronésiennes (infographie C. Jeunesse).

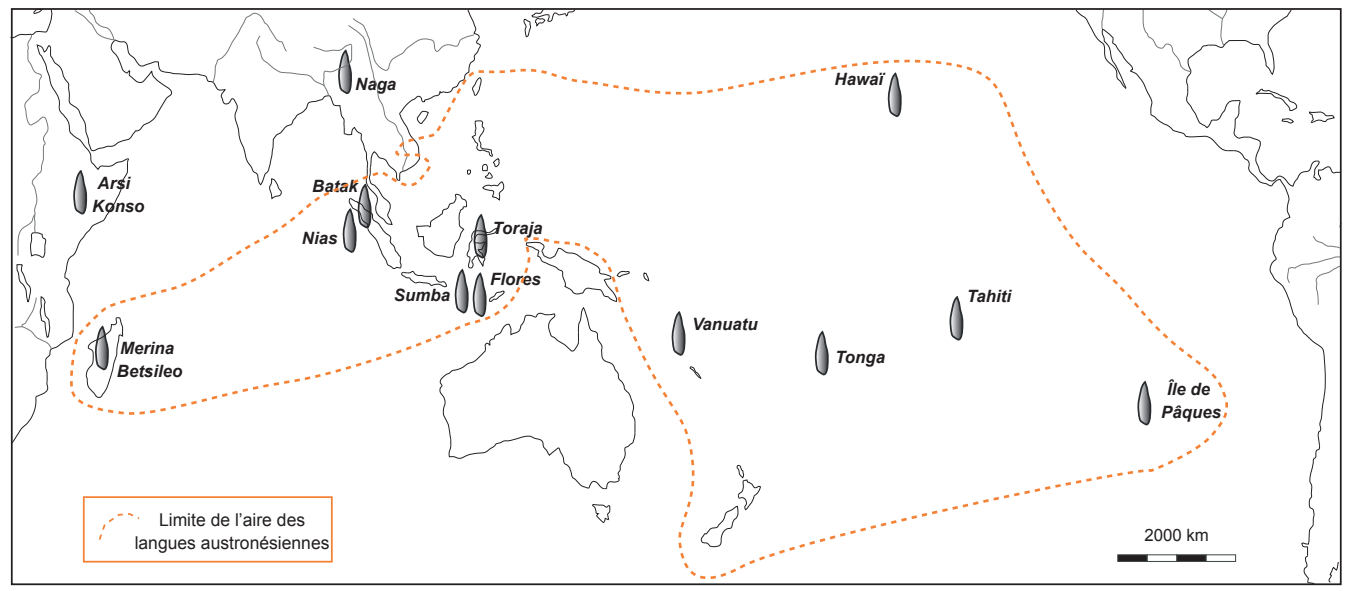

Figure 2: Pierre dressée à l'occasion d'une fête du mérite dans le village de Maram, en pays Naga (Assam, Inde) (d'après Flannery et Marcus 2012).

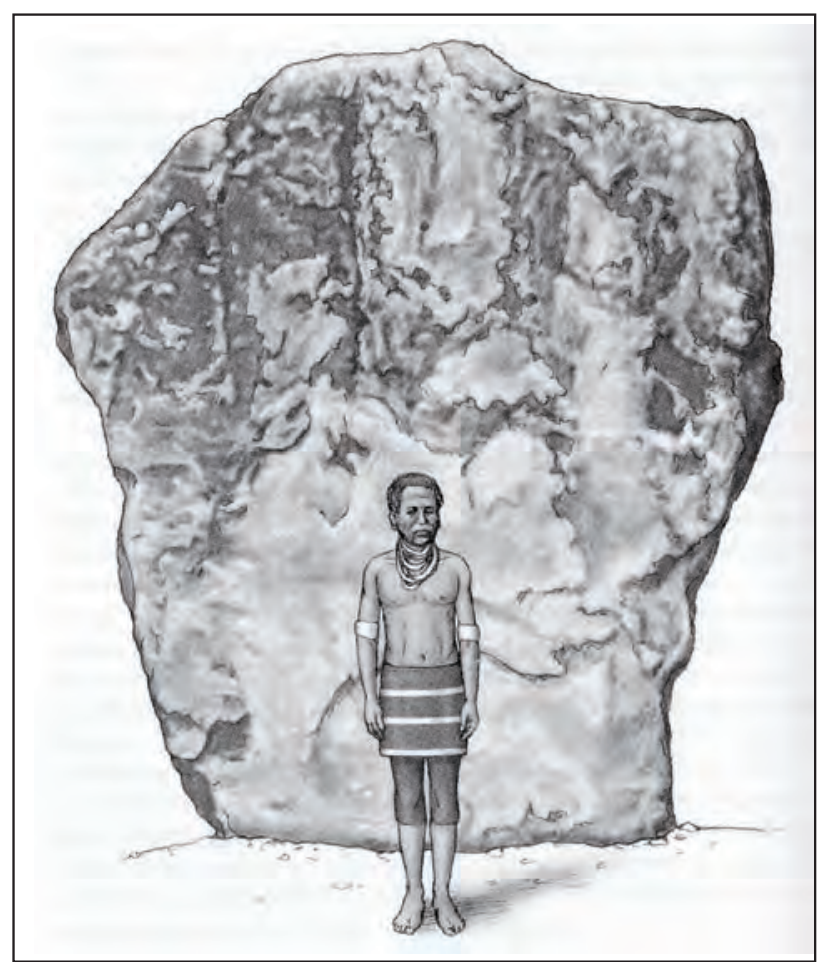


Figure 3 : Reconstitution d'un Marae de Tuamotou, Polynésie française (d'après Renfrew 1984).

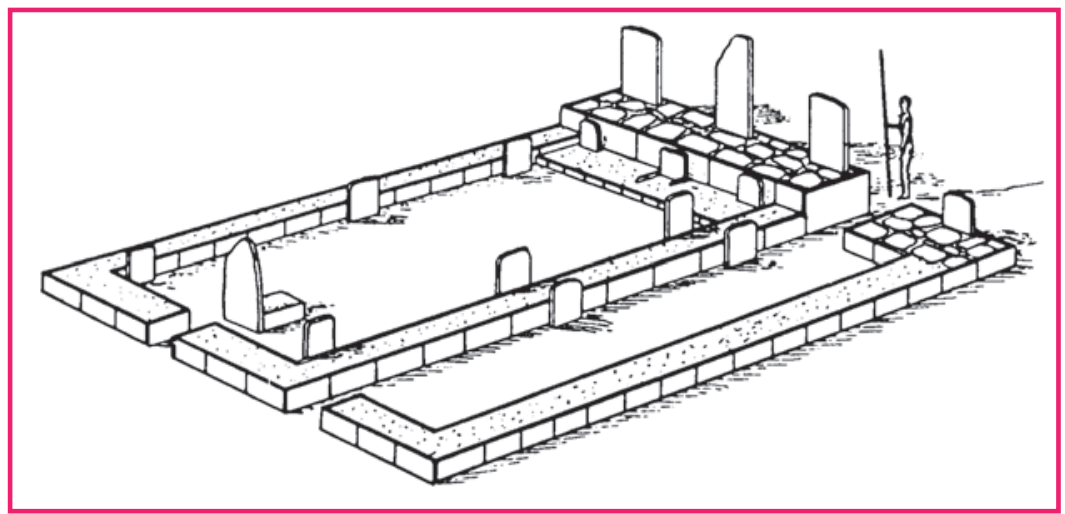

Figure 4 : Menhir «habillé » à vocation funéraire Gewada (Éthiopie) photographié par Jean-Paul Cros dans les années 2000 (copyright : J.-P. Cros).

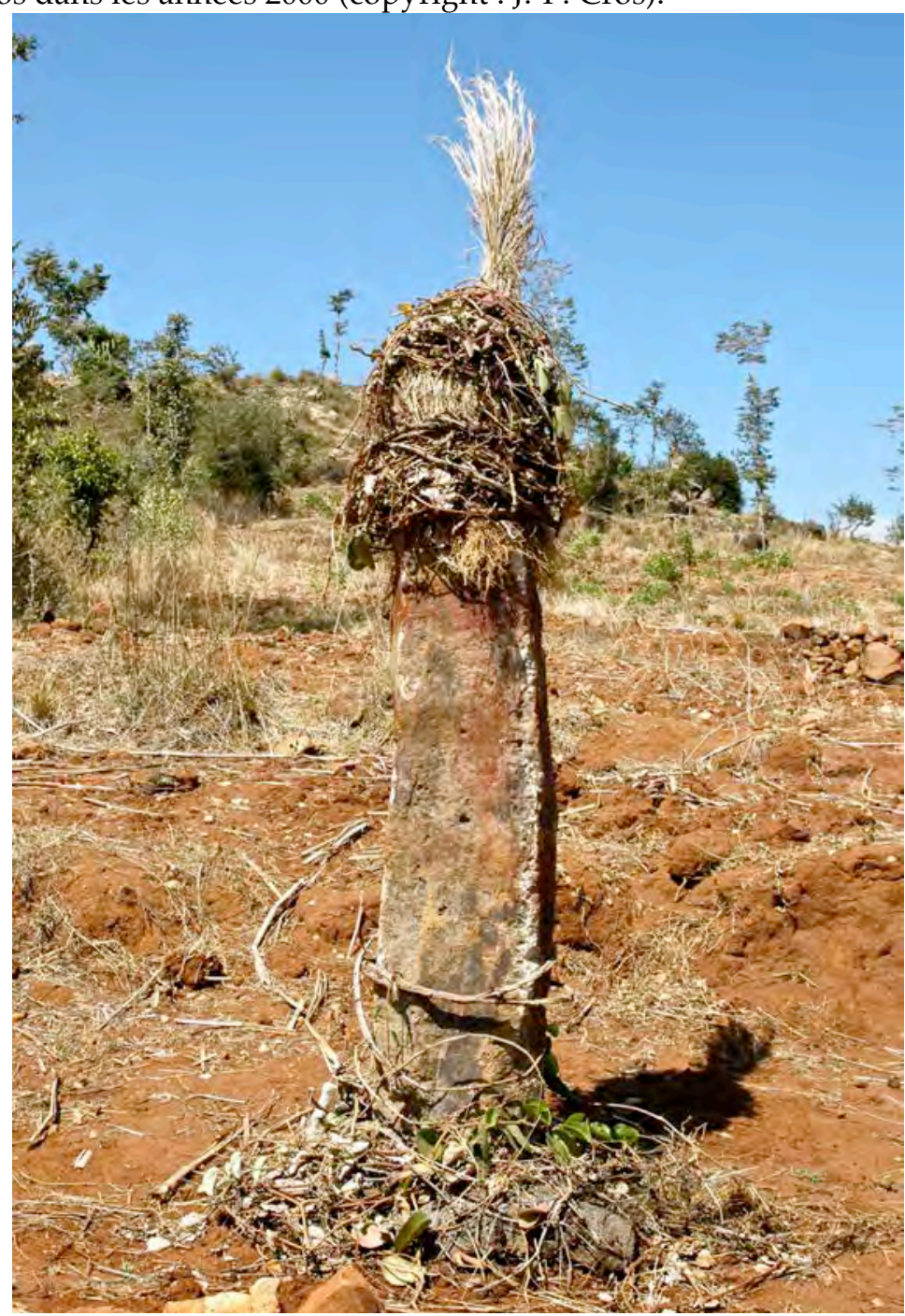




\title{
Les signes "à valeur ajoutée " dans l'expression graphique schématique du Néolithique
}

\author{
Philippe HAMEAU \\ Maître de Conférences en Anthropologie, \\ Université de Nice-Sophia Antipolis, MSHS Nice \\ \begin{tabular}{r}
\hline Extrait de : Olivier BUCHSENSCHUTZ, Christian JEUNESSE, Claude MORDANT et Denis VIALOU (dir.), \\
Signes et communication dans les civilisations de la parole, Paris, \\
Édition électronique du CTHS (Actes des congrès des sociétés historiques et scientifiques), 2016.
\end{tabular} \\ Cet article a été validé par le comité de lecture des Éditions du CTHS dans le cadre de la publication \\ des actes du $139^{\mathrm{e}}$ Congrès national des sociétés historiques et scientifiques tenu à Nîmes en 2014.
}

\begin{abstract}
Résumé
Le corpus iconographique du Néolithique, peint ou gravé, est restreint à quelques catégories de figures: les êtres vivants (hommes, animaux et "idoles») et les signes (motifs soléiformes et lignes brisées). Les uns et les autres peuvent être figurés dans des versions très réalistes, au sens d'immédiatement reconnaissables, ou très simplifiées au point de nous paraître absconses. La syntaxe de cette iconographie est perceptible au travers de règles précises d'apposition des figures (emplacement, taille et translation) et de leurs associations par juxtaposition ou contraction. Ces processus associatifs nous conduisent à penser que les motifs soléiformes et les lignes brisées indiquent le statut des êtres vivants : l'étape de leur passage et transformation sur et par le site sur lequel ils sont figurés. Il semble que cette même valeur ajoutée véhiculée par les mêmes signes soit reprise pour l'iconographie linéaire médiévale. Ceci explique en partie que les mêmes sites sont souvent marqués aux deux époques et infère un comportement syntaxique inhérent au schématisme.
\end{abstract}

Mots-clés

Schématisme, écriture, signe soléiforme, ligne brisée, Néolithique, Sud de la France

\begin{abstract}
The iconographical collection from the Neolithic, including paintings and engravings, has a small number of figures, mainly represented by living beings (people, animals and idols) and symbols (sun motifs and broken lines). These figures can be realistic and immediately recognisable, or can be simplified until appearing quite abstract. The iconographical syntax is perceived using precise rules of figure layout (position, size and translation) and their association by juxtaposition or contraction. These associations indicate that the sun motifs and the broken lines represent the status of the living beings : a stage of their passage and their transformation at and influenced by the site where they are figured. It seems that the same message was transmitted by the same symbols in medieval linear iconography. This explains in part why the same sites were marked during these two periods and infers actions based on syntax and inherent to established schemas. (traduction Rebecca Peake)
\end{abstract}

\section{Une écriture sélective}

Pendant les $\mathrm{IV}^{\mathrm{e}}$ et $\mathrm{III}^{\mathrm{e}}$ millénaires av. J.-C., une expression graphique dite schématique envahit l'univers matériel et idéel des communautés agropastorales du sud-est de la France et de la péninsule Ibérique. Le terme expression graphique remplace ici celui d'art, régulièrement employé par les chercheurs et par l'Administration de la discipline on parle encore d'art postglaciaire : le programme P30 de la Recherche archéologique afin de souligner l'existence d'une syntaxe, c'est-à-dire d'un système unissant les motifs entre eux pour qu'ils évoquent des thèmes.

Cette expression graphique aurait pu être rangée dans les écritures pictographiques car elle est essentiellement constituée de motifs simples et géométriques. Toutefois, la 
diversité des versions formelles d'une même figure est telle qu'il ne semble pas y avoir de pictogrammes récurrents et stables. En fait, il existe un très petit nombre de figures de base mais elles sont sujettes à des transformations formelles parce qu'elles sont interprétées à titre individuel ou au niveau d'un groupe humain restreint, d'où la multiplicité de leurs versions graphiques.

À ce titre, on parle souvent d'expression schématique puisqu'il est possible d'exprimer la même charge sémantique avec une figure réaliste, au sens d'une figure identifiable, ou avec un motif très simplifié en passant par toutes les versions intermédiaires entre ces deux extrêmes (fig.1). Les multiples versions d'un signe ne signifient aucunement une évolution d'ordre chronologique. On ne saurait interpréter ces variations morphologiques comme des indices de typo-chronologie comme on l'a longtemps fait en parlant de figures «sub-réalistes », "proto-schématiques » par exemple. Il est vrai qu'on a aussi et jusqu'à récemment expliqué l'évolution des expressions graphiques du Paléolithique à partir des mêmes considérations, des figures les plus frustes aux plus abouties, acceptant implicitement le primat de l'esthétique sur le contenu sémantique de ces représentations.

Dans le même temps, le qualificatif de schématique qui sert à particulariser l'expression graphique néolithique n'est pas très approprié. La réduction graphique est un phénomène qui affecte toutes les expressions, en tous lieux et à toutes les époques, du Paléolithique à aujourd'hui. Il s'agit en fait d'abréger la forme du signifiant, d'utiliser la synecdoque, les abréviations et autres simplifications dans les limites de ce qui est culturellement compréhensible puisqu'il s'agit de la communication d'une pensée. En conséquence, il n'est pas sûr que l'expression graphique néolithique soit plus schématique que les autres au point d'ériger en spécificité la simplification de ses traits.

On pourrait également la ranger au nombre des écritures sélectives au sens que P. Déléage donne à ce terme : des écritures qui ne codent qu'une partie du discours et qui ne se comprennent que si elles sont contextualisées (Déléage 2013). En effet, l'expression graphique dont il est question est essentiellement restreinte à cinq figures lesquelles représentent environ $95 \%$ de l'ensemble des motifs. Les $5 \%$ restants correspondent à des motifs particuliers à un site ; ils ne sont pas récurrents et ils n'entrent pas a priori dans les catégories usuelles. De même, il semble bien que la fréquentation et l'usage des sites marqués par cette expression correspondent au concept de "passage et transformation » (Hameau 2002). Quand leur usage est explicite, ce sont des sites sépulcraux (grottes sépulcrales, dolmens) ou bien des abris au niveau desquels nous pensons que se sont déroulés des rites d'initiation ${ }^{1}$. Or, la mort et l'initiation sont par essence des rites de passage et de transformation sociale. À ce titre, les sites peints sont peut-être plus des sites marqués que des sites ornés. La finalité de leur fréquentation n'est peut-être pas de les peindre et l'on peut imaginer qu'au cours de diverses pratiques en lien avec l'initiation, les visiteurs soient amenés à exprimer, succinctement, leur propre transformation. Le signe ne serait que le vestige le plus pérenne ou le plus évident d'un ensemble de pratiques sociales ayant contribué à son élaboration. En conséquence, l'expression graphique étudiée ici pourrait se satisfaire d'une iconographie restreinte pour évoquer l'essentiel de ce contexte ritualisé. En cela, elle serait sélective.

C'est donc en fonction de ces considérations que nous envisagerons la présence du motif soléiforme et de la ligne brisée, dans l'expression graphique du Néolithique, toutes techniques et tous contextes confondus. Si les mêmes motifs et les mêmes associations de motifs sont exprimés par la peinture pariétale, la gravure rupestre, la sculpture (les fameuses statues-menhirs) et sur support mobilier, il faut donc considérer l'expression graphique de la fin du Néolithique comme un ensemble cohérent: ce que certaines équipes espagnoles préfèrent appeler «art mégalithique »: les travaux de Primitiva Bueno, par exemple.

1. Nous avons inventorié l'ensemble des éléments qui nous permettent de conclure à de tels rites dans Hameau 2010a. 


\section{L'essentiel du corpus}

Le nombre des catégories de motifs est donc très faible (fig.2). D'ailleurs, dans toute expression graphique, le réservoir symbolique est généralement réduit et il n'est pas étonnant qu'il le soit ici. Ces catégories de motifs ${ }^{2}$ sont des personnages masculins qui, par schématisation et simplification, deviennent des signes anthropomorphes masculins ; des quadrupèdes, essentiellement des cervidés, plus rarement des caprinés, qui peuvent être réduits à l'expression de leurs appendices frontaux, bois ou cornes ; des idoles, c'està-dire des représentations anthropomorphes qui ne sont pas l'homme, qui appartiennent plutôt à un niveau qualifiable de supranaturel, et qu'on simplifie par l'indication des traits du visage ( $\mathrm{T}$ facial avec ou sans yeux), par celle de la forme générale (motif en arceau) ou par celle de son principal attribut (le collier). Enfin, ce sont des signes soléiformes faits de rayons qui se recoupent ou qui partent d'un cercle central et qui peuvent être réduits à de simples points en peinture ou à des cupules en gravure ; des lignes brisées verticales ou horizontales, parfois emboîtées jusqu'à devenir de véritables résilles, parfois simplifiées jusqu'à n'être plus que des chevrons.

À la suite de ces catégorisations, quelques remarques s'imposent. D'abord, trois de ces catégories correspondent à des motifs que l'on peut interpréter comme des êtres vivants (personnage, quadrupède, idole) au contraire des deux autres. Or, ce sont ces derniers motifs qui accompagnent indifféremment les trois premiers. Ensuite, il apparaît que ce que nous appelons simplification ne signifie pas nécessairement réduction des éléments caractéristiques de la figure. Ainsi, le personnage peut devenir un motif arboriforme par multiplication de ses membres de même que des lignes brisées peuvent devenir des résilles par réplication et emboîtement de leurs formes. Enfin, personnages et idoles peuvent être dotés d'objets qui finissent par devenir leurs véritables attributs : arc, bâton, canne, coiffe de plumes par exemple pour le personnage masculin ; arc, "objet », collier, hache, crosse... pour l'idole. Ces attributs sont rares, ils ne sont pas systématiques et ils sont parfois exprimés à la place du personnage ou de l'idole.

Pour passer des motifs aux thèmes, il faut qu'il y ait association d'au moins deux motifs ${ }^{3}$. Des règles président à ces associations. Les motifs peuvent être juxtaposés. La récurrence de leur proximité est conçue comme l'indice de leur complémentarité. Les motifs peuvent également être imbriqués. À partir de deux motifs différents, on façonne une nouvelle figure qui les intègre. C'est sans doute l'imbrication des motifs qui garantit le mieux notre sentiment d'un lien profond et sémantique entre eux. En effet, de nombreux exemples ethnographiques démontrent que des relations peuvent être plus étroites entre des motifs distants qu'entre des motifs proches mais nous n'avons aucun moyen d'en faire la preuve pour le présent corpus.

Ces associations, surtout pour celles qui utilisent la juxtaposition, ont été signalées de longue date par divers chercheurs sans que ceux-ci soient nécessairement allés plus loin que cette simple observation. La plupart ont vu qu'il existait un lien entre le motif soléiforme et l'homme, le cervidé ou encore l'idole.

«N'importe quel connaisseur de l'art schématique péninsulaire concéderait le poids spécifique de l'association élément anthropomorphe/soleil, dans toutes les versions possibles. $»^{4}$

2. Personnages masculins, quadrupèdes et idoles ont fait l'objet de plusieurs articles thématiques (respectivement, Hameau 2013, 2006, 2003 et à paraître). La présente étude se conçoit donc comme la suite logique des précédentes.

3. Même si en fin d'article, nous postulons que l'un des deux motifs peut n'être que mentalement présent.

4. Bueno Ramirez et Balbin Behrmann 2000, p. 446. 
Les exemples proposés par J.L. Sanchidrián Torti et V.E. Muñoz Vivas (1983, p. 163) comme éléments de comparaison avec certaines peintures post-paléolithiques de la cueva de la Pileta (Málaga) sont un modèle du genre. La ligne brisée comme élément associé n'a pas fait l'objet d'une telle démonstration.

J. Martinez Garcia (1984) pense aussi qu'existent des associations récurrentes, mais il signale surtout la relation homme/animal et propose une lecture très structuraliste des panneaux en termes d'associations symboliques, d'inversions symétriques, de dichotomies normatives. La teinte des figures elle-même lui semble une stratégie dans ces oppositions sémantiques qu'il pense être un indice du système économique des populations qui ont marqué les parois.

Il apparaît pourtant que les associations de motifs sont autres et plus complexes. D'abord, le motif soléiforme et la ligne brisée sont si systématiquement placés près des personnages, des quadrupèdes et de l'idole que l'on peut s'étonner qu'ils soient totalement absents des exemples donnés par J. Martinez Garcia. De même, le code chromatique qu'il propose pose le problème de la stricte contemporanéité des motifs humains et animaux. Généralement, des teintes différentes suggèrent des compositions pigmentaires et/ou des moments graphiques différents. L'analyse de paires de motifs strictement synchrones est donc préférable. En fait, à travers ces jeux d'association de motifs conçus comme des éléments syntaxiques, J. Martinez Garcia veut exprimer le lien de subordination des animaux à l'homme pour démontrer qu'il s'agit bien d'une expression graphique néolithique au sens d'une économie basée entre autres choses sur l'élevage.

Toutefois, un autre cas d'association existe qui n'est jamais signalé comme tel, celui qui consiste à doubler la même figure (fig. 3). Or, les cas de doublement sont très fréquents et ils concernent indifféremment les trois catégories d'êtres vivants. Les exemples valent quelles que soient les techniques d'expression utilisées, peinture, gravure ou sculpture. Ce doublement est le plus souvent imparfait: les deux motifs ont des sens de lecture différents, ou bien l'un est plus petit que l'autre, ou encore l'un est seul tandis que l'autre est associé à un motif soléiforme ou à une ligne brisée.

Dernière observation concernant la thématique et les associations de motifs : lorsqu'il y a contraction de deux motifs en un, elle ne porte jamais sur l'imbrication de deux figures d'êtres vivants. On ne connaît pas de personnage ou d'idole dont la tête porterait des bois de cervidés, et il n'existe pas de cervidé à tête d'homme ou d'idole. La contraction se fait uniquement entre un motif représentant un être vivant et un motif soléiforme ou une ligne brisée. Ces remarques préalables permettent donc d'appréhender la place que peuvent occuper ces deux derniers motifs.

\section{Modes d'association avec le signe soléiforme et la ligne brisée}

Le motif soléiforme adopte lui-même de nombreuses morphologies, depuis la figure rayonnante avec ou sans cercle central jusqu'au simple point, s'il s'agit de peinture, ou jusqu'à la cupule, s'il s'agit de gravure. C'est même sous sa forme ponctuée ou cupulée qu'il est le plus fréquent pour des raisons évidentes de rapidité d'exécution. Dans le cas du point peint, on a même imaginé que la gestuelle, le fait de toucher la paroi du bout des doigts, avait tout autant d'importance que le fait de tracer la figure dans la totalité de ses traits.

Les stèles venaissines du Rocher des Doms à Avignon et de l'Isle-sur-Sorgue soulignent cette correspondance entre le motif rayonnant et la ponctuation, et entre le signe isolé et le signe répété (Gagnière et Granier 1976, fig. 23-26). La contraction du motif soléiforme avec les êtres vivants revêt souvent un caractère esthétique. Ainsi, les deux yeux de l'idole peuvent prendre la forme de deux soleils, ce qu'on observe fréquemment sur les 
figurines ibériques d'idoles sur plaquettes de schiste ou sur la petite statuette découverte dans la mine de Gavà (Catalogne) (Bosch et Estrada 1994, p. 287-291). Un point accompagne souvent le personnage mais deux points peuvent aussi l'encadrer par simple souci d'équilibre. Assez fréquemment, ces points sont interprétés, selon leur emplacement, comme les yeux, les seins ou les testicules du personnage alors qu'ils ne sont positionnés ainsi qu'en fonction de la symétrie droite/gauche des individus considérés de face. L'interprétation réaliste des signes oublie que la motivation graphique n'est pas nécessairement iconique, qu'il n'y a pas forcément une correspondance entre la forme et ce qui est représenté (Harris 1993). En revanche, s'agissant de formes tracées pour exprimer un message, diverses stratégies peuvent présider à leur apparence, depuis le regroupement des motifs jusqu'à leur dislocation, ces deux versions extrêmes n'écartant pas obligatoirement leur beauté plastique (Boas 2003).

D'autres exemples du rapprochement de l'être vivant avec le motif soléiforme existent encore, très différents les uns des autres dans leur forme, comme les motifs soléiformes dont les rayons sont hérissés d'appendices à la façon de bois de cervidé (Tajos de las Figuras, Cadix, Abelanet 1986, fig. 18), le personnage à tête soléiforme de l'abri Neukirch (Var, Hameau 2000, fig. 103) ou bien les traits verticaux multirenflés (Los Buitres, Badajoz) que H. Breuil (1933/35, vol. III, fig.17) appelait des barres humaines.

L'association de la ligne brisée et de la représentation d'un être vivant offre un peu moins d'exemples diversifiés. Dans quelques rares cas, la ligne brisée semble bien symboliser l'élément liquide. Ainsi, à l'abri d'El Gabal (Alméria), un cerf incline nettement la tête comme s'il s'abreuvait, l'eau étant représentée par quatre lignes brisées allongées (Breuil 1933/35, vol. IV, pl. XXIV) Ce motif en ligne brisée se fond dans la représentation de certaines idoles sous la forme de chevrons qui deviennent leur coiffe ou bien le décor de leur cape (Breuil 1933/35, vol. IV, pl. XI). La contraction de la ligne brisée avec le personnage masculin se traduit parfois par des individus dont les membres inférieurs s'allongent en longues lignes brisées (Estrecho de Santonge, Almeria, (Breuil 1933/35, vol. IV, pl. XXV) ou bien dont les mêmes membres prennent une forme en $M$ qui a souvent été interprétée comme leur position accroupie (Pena Escrita de Fuencaliente, Breuil 1933/35, vol. III, pl. XLIV). En fait, dans ces deux exemples, seul change le développement, long ou court, de la ligne brisée.

\section{Les compositions plus complexes}

Au-delà des interprétations réalistes qui sont aussi les plus fréquemment proposées, mais qui ne tiennent nullement compte de la variabilité symbolique, l'herméneutique des motifs, soléiforme et ligne brisée, ne peut être appréhendée qu'en observant les contextes et la place de ces figures dans des ensembles iconographiques plus complexes. À ce titre, le panneau de la rotonde sud de Baume Peinte (Vaucluse) apporte quelques éléments significatifs (fig. 4a).

Les figures de cet ensemble sont parfaitement synchrones. Les analyses stylistiques, incluant un travail sur la gestuelle ayant présidé au tracé des motifs, et techniques permettent de conclure à un panneau réalisé par le même individu ayant utilisé le même pinceau et le même mélange pictural (Hameau 1997, 2009, 2012). Il n'y a ni ajouts, ni repeints, ni repentirs qui pourraient laisser imaginer plusieurs logiques symboliques accumulées au fil du temps. La cohésion sémantique de l’ensemble est donc avérée.

Il s'agit d'une composition sur deux registres séparés par un axe horizontal ponctué. Audessous de l'axe, les figures sont en position couchée ou aberrante. La position du cervidé $\mathrm{n}^{\circ} 14 \mathrm{n}^{\prime}$ est pas réaliste, par exemple. Au-dessus de l'axe, les figures sont en position droite. Elles sont parfois doublées et quand il y a doublement, une figure est ponctuée et $l^{\prime}$ autre ne $l^{\prime}$ est pas. C'est le cas des arceaux $n^{\circ} 15$ et 18 et des signes losangiques $n^{\circ} 21$ et 25 . Pour les arceaux censés représenter l'idole, il y a même contraction puisque le contour 
d'un de ces motifs est ponctué. Dans le cas des signes losangiques, il y a juxtaposition des points et du losange, mais les axes de symétrie de la figure entraînent l'apposition de plusieurs points. Ce signe losangique existe sous une forme plus anthropomorphisée puisque pourvue de membres inférieurs à la grotte Monier (Var) (Hameau 2002, fig. 143.1). On constate donc ici que la ligne ponctuée agit comme une délimitation et que les ponctuations associées à des figures n'appartiennent qu'au registre supérieur.

À cette opposition haut-bas, s'ajoute une opposition gauche-droite. En effet, le support de ce panneau étant une rotonde de 2,50 m de diamètre, ses deux extrémités se retrouvent en vis-à-vis. Or, aux figures $n^{\circ} 1,2$ et 3 , couchées et/ou non ponctuées répondent les figures $n^{\circ} 25,28$ et 30 , droites et/ou ponctuées. Cette opposition vaut parce que ces différentes figures sont placées avant et après l'axe ponctué.

Il apparait donc que les êtres vivants de cette composition sont diversement orientés, accompagnés et spatialisés, selon la place qu'ils occupent par rapport à la ligne de points. Ces derniers, en tant que signes soléiformes, semblent donc représenter le symbole et le véhicule du passage.

\section{La valeur ajoutée}

Au vu de cet ensemble iconographique où la place de chaque motif n'est ni le produit du hasard, ni imposée par la nature ou par l'état du support, nous supposons que le motif soléiforme, complet ou simple point, rajoute un sens à la figuration de l'être vivant près de laquelle il est placé ou avec laquelle il est imbriqué. Il agirait donc comme un déterminant. Si l'expression graphique étudiée ici est à mettre en relation avec des rites de passage et de transformation sociale, le motif soléiforme, pourrait bien indiquer le nouveau statut de l'être vivant. Il constituerait alors un signe « à valeur ajoutée ».

Les doublements d'un même être vivant sont imparfaits (fig. 4 b-e). Une idole ou un personnage est accompagné d'un point et l'autre ne l'est pas. Ces motifs en binôme représentent peut-être deux états de cet être vivant. Pour Baume Peinte, ce sont les deux arceaux ou les deux signes losangiques. D'innombrables versions graphiques de ces doublements imparfaits sont d'ailleurs possibles. C'est ainsi que sur les pentes du Signal de la Lichère (Gard), site à gravures rupestres, le double arceau finit par devenir un signe en $\mathrm{M}$ dont un seul des jambages abrite une cupule (Hameau 2002, fig. 200). Au Puerto Alonzo (Badajoz), le doublement du même personnage masculin veut qu'un seul des deux motifs ait un corps multirenflé (Breuil 1933/35, vol. II, fig. 23.e).

La ligne brisée semble bien avoir la même fonction déterminative. Elle aussi, accompagne imparfaitement de nombreux cas de doublement, essentiellement le doublement du personnage. Ce sont les personnages censément accroupis de la Piedra Escrita de Fuencaliente systématiquement flanqués d'un personnage debout (Breuil 1933/35, vol. III, pl. XLIV) ou bien ces personnages doublés dont l'un des deux est poursuivi par une ligne brisée sur le site de La Graja (Jaen) (Breuil 1933/35, vol. III, fig. 41).

Il n'est d'ailleurs peut-être pas besoin d'exprimer les deux états, pas besoin de les opposer l'un à l'autre. Si l'on admet que l'abri peint constitue vraiment le lieu du passage, on peut admettre que compte surtout l'indication de la transformation de l'être vivant ou même l'indication du concept de transformation, c'est-à-dire la finalité de sa présence sur le site. Concernant les rites de passage, opposer l'avant et l'après rite est surtout conforme au schéma proposé par A. Van Gennep (1909). P. Bourdieu lui préfère parler de « rites d'institution » en arguant que le principal de ce rite n'est pas de dissocier en deux temps ceux qui le font, mais plutôt d'opposer ceux qui le font et ceux qui ne le font pas (1982). Ainsi, pourrait-on comprendre que l'on ait très souvent, sur les sites, des personnages ou des idoles en exemplaire unique accompagnés d'un motif soléiforme ou d'une ligne brisée. Ainsi, pourrait-on également concevoir que les motifs soléiformes ou 
les lignes brisées ne soient pas accompagnés de motifs d'êtres vivants. Ce cas est même le plus répandu : la plupart des pierres à cupules n'expriment que ce motif sur leur support, le réseau supérieur de la grotte de l'Église à Baudinard (Var) n'abrite que des motifs soléiformes (Hameau 2002, fig.105 et 106) de même que la ligne brisée est presque le seul motif peint sur plusieurs sites du département de la Drôme (abri 2 de Pierre Rousse, abri d'Eson, Baume Ecrite) (Hameau 2002, fig. 29, 33, 34, 36).

En conséquence, la réduction graphique telle qu'envisagée pour les motifs et qui vaut à cette expression graphique le qualificatif de schématique semble valoir aussi pour les thèmes. Il semble que l'on peut présenter la totalité du thème sous une forme réaliste ou identifiable, mais que l'on peut aussi simplifier à l'extrême ce thème en ne gardant plus que le symbole indispensable à sa compréhension: ici, le motif soléiforme ou la ligne brisée. Après tout, au moment où il les trace sur la pierre, l'auteur sait très bien que c'est à lui ou à ses condisciples du moment que s'adressent ces figures et il en connaît la charge sémantique. Il peut donc résumer son message par le tracé de ces seuls motifs. C'est d'ailleurs le fait des écritures sélectives de ne retenir que les faits saillants et incontournables.

\section{Valeur ajoutée et longue tradition graphique}

Une fois posée l'hypothèse d'un système de signes « à valeur ajoutée » dans l'expression graphique de la fin du Néolithique, il semble que d'autres corpus iconographiques connaissent des processus d'associations de motifs dans les mêmes termes et en utilisant parfois les mêmes symboles. Bien sûr, nous réaffirmons notre conception relativiste du symbole qui veut qu'un même motif n'a pas automatiquement la même signification partout et à toutes époques. En revanche, les mécanismes syntaxiques présentés plus haut tels que simplification, juxtaposition, contraction, translation, etc., existent pour d'autres expressions graphiques même si les chercheurs leur attribuent d'autres noms tels que stylisation, association, abréviation, rotation, etc.

Ainsi, motifs soléiformes et lignes brisées existent dans d'autres corpus peu éloignés du point de vue des dates de celui que nous avons étudiés : les gravures du Mont Bégo ou du Val Camonica par exemple (de Lumley 1995, Priuli 1989). Ils sont parfois associés, voire imbriqués, avec des motifs représentant des êtres vivants tracés sous diverses formes : ponctuation à proximité d'un personnage masculin, motif corniforme dont les cornes encadrent un point, personnage aux bras en ligne brisée, motif corniforme dont les cornes sont en lignes brisées par exemple (de Lumley 1995, fig. 26, 28,102 ; Priuli 1989, p. 39). On peut penser qu'ils sont des signes à valeur ajoutée, même si l'on est conscient que cette valeur ajoutée n'est peut-être pas identique à celle que nous avons proposée pour le corpus iconographique précédent. Ainsi, en signalant les points et les traits courts tracés près des motifs anthropomorphes de la Grotta Scritta 1 à Olmetta-du-Cap en Corse, MC.Weiss fait l'inventaire de quelques interprétations qui sont parfois faites de ces motifs : désigner le personnage humain le plus remarquable, signaler les pensées de l'individu, etc. $(2003 \text {, fig. } 14)^{5}$

Une expression graphique d'époque historique ${ }^{6}$, dite schématique linéaire - d'autres chercheurs l'appellent art filiforme -, reprend les mêmes mécanismes syntaxiques et une grande partie des motifs énumérés pour le Néolithique (Hameau 2001). Cette expression graphique est composée de motifs réalisés sous abris ou sur rochers par incision superficielle de la roche.

5. M.C.Weiss reprend certaines des interprétations données par différents auteurs au sujet de plusieurs ensembles iconographiques. À ce titre, il ne serait pas concevable d'assigner des sens différents à un même motif considéré au sein d'un même corpus. En ce cas, toute la démonstration faite ici s'effacerait au profit d'un travail au cas par cas.

6. Cette expression historique correspond à une grande amplitude de temps qui inclut le Moyen Âge. Selon les auteurs, elle commence à la fin de l'âge du Fer et/ou perdure jusqu'au début du XX⿳亠丷厂犬⿱ 
Au nombre de ces motifs, des personnages masculins et féminins sont parfois flanqués d'un signe soléiforme rayonnant ou d'une ligne brisée (fig. 5). Soumis aux mêmes simplifications que celles que nous avons vues pour le Néolithique, ces personnages deviennent des motifs anthropomorphes dits arboriformes, en flèche, etc. Des effets de contraction existent également et se traduisent par des personnages ou des signes anthropomorphes aux yeux soléiformes, à tête soléiforme ou bien aux membres en ligne brisée. Or, cette expression schématique linéaire d'époque historique investit parfois des abris déjà peints ou des rochers déjà gravés au Néolithique : à ce jour, une vingtaine de sites sont concernés par ce double marquage dans le sud-est de la France. Elle le fait selon plusieurs stratégies. Les gravures linéaires restent en marge des peintures préhistoriques, ou bien elles répliquent les motifs préhistoriques en usant parfois d'une version formelle différente (un signe soléiforme linéaire sur une cupule préhistorique, par exemple) ou encore, elles complètent les motifs préhistoriques en rajoutant aux personnages néolithiques le signe à valeur ajoutée qui n'y était pas ou qui a disparu avec le temps (un signe soléiforme linéaire rajouté à la représentation d'un personnage masculin, par exemple) (fig. 5e).

Les graveurs se sont donc réappropriés les lieux mais aussi le corpus iconographique déjà présent. Compte tenu du hiatus chronologique, l'herméneutique de l'ancien corpus n'a sans doute pas été comprise mais les graveurs ont répondu à deux processus mentaux. En premier, ils ont opéré la longue tradition graphique des lieux qui veut qu'un site déjà marqué soit aussi un site remarquable parce qu'il porte en lui un usage qui lui est particulier. Ils ont également conçu l'efficacité symbolique des motifs au sens que $\mathrm{Cl}$. Lévi-Strauss (1958) et M. Eliade donnent respectivement à ce terme :

«Le symbole délivre son message et remplit sa fonction alors même que sa signification échappe à la conscience. $»^{7}$

Ceci est possible parce que les deux corpus correspondent à un même système syntaxique avec des motifs principaux et des motifs à valeur ajoutée et que la forme des signifiants utilisés est identique.

\section{La prise en compte des mêmes motifs}

Considérer l'expression schématique de la fin du Néolithique comme une écriture sélective a permis d'appuyer la démonstration sur l'importance de la contextualisation des données graphiques, c'est-à-dire les conditions culturelles et sociales dans lesquelles le support a été marqué, et sur l'idée que ces figures sont des éléments de langage et qu'à ce titre, des mécanismes président à leur apposition sur ce même support de façon qu'elles soient compréhensibles par le plus grand nombre. Passer du contexte de l'initiation au concept de "passage et transformation » permet d'expliquer que les signes «à valeur ajoutée » ne concernent pas uniquement les représentations de personnages masculins. Les quadrupèdes et l'idole passent aussi et se transforment et l'on peut sans doute étendre cette notion au site lui-même qui, de lieu naturel, abri ou rocher, devient monument, et aux matières premières, silex, argile, serpentinite, par exemple, lorsqu'elles sont extraites et transformées sur place ${ }^{8}$. Envisager la présente iconographie comme un vecteur de communication suppose que les motifs n'expriment une idée que s'ils sont liés à d'autres motifs. Cette posture évite de recourir à des typologies de catégories de figures toujours plus importantes et qui ne font que confirmer l'extrême variabilité des formes d'un motif sans pouvoir, compte tenu du recul du temps, donner un sens à une version graphique plutôt qu'à une autre. En revanche, la connaissance des mécanismes d'association de motifs, juxtaposition et contraction, permet de ne pas confondre figure et

7. Eliade 1965, p. 97.

8. Une quinzaine d'abris peints du sud-est de la France sont des gîtes de matière première, par exemple. 
unité graphique (Vialou 1987) tout en acceptant que des versions graphiques jouent sur la symétrie et le rythme, deux éléments inhérents à l'« art primitif » (Boas 2003).

On observe aussi que les modes d'abstraction de cette expression graphique existent à différents niveaux de représentation et que l'on peut simplifier, indifféremment, les motifs et les thèmes. Les exemples les plus aboutis d'associations de figures, au sens des plus explicites, connaissent eux-mêmes une schématisation de leurs éléments si bien que le motif de l'être vivant peut ne pas être doublé, voire même que la seule valeur ajoutée soit exprimée en tant que finalité du passage sur et par le site.

Enfin, les mécanismes décrits ici semblent régir d'autres expressions graphiques. L'expression linéaire a servi d'exemple en ce qu'elle donne aux lieux leur concrétude : un usage des mêmes sites, des mêmes supports, des mêmes motifs et de la même syntaxe reliant deux corpus iconographiques pourtant éloignés dans le temps et dans leur contenu.

En l'occurrence, l'usage des mêmes motifs «à valeur ajoutée » peut surprendre. Cependant, certains symboles sont retenus plus que d'autres en raison de la charge sémantique qu'ils véhiculent même si cette charge est distribuée selon des valeurs constrastives. Cl. Lévi-Strauss explique que :

«Les récits mythiques de par le monde mettent en place des milliers de soleils profondément différents et même contradictoires d'une culture à l'autre. Reste que cette multiplicité se greffe sur une expérience qui, quelle que soit la variété des contextes, confère à cet astre, comme à nul autre, une richesse sémantique et un statut singulier qui lui permettent cette abondance de combinaisons narratives. $»^{9}$

Un inventaire des logos contemporains utilisant le soleil comme motif de base aboutirait de même à une très longue liste de concepts tout aussi diversifiés et la ligne brisée offrirait tout autant d'interprétations. Pour être complet sur la polysémie des signes, il faut aussi admettre que si ces derniers acquièrent un sens particulier en fonction du contexte précis dans lequel ils sont exprimés, leurs autres significations subsistent mais restent simplement en arrière-plan (Coquet 2011).

Dans l'expression graphique du Néolithique, la prépondérance numérique du signe soléiforme et de la ligne brisée - à eux deux, ils représentent $80 \%$ du corpus exprimé - et leur importance sémantique admettent peut-être quelques analogies dans le contexte même des sites marqués par ces motifs. Ces lieux répondent en effet à quatre paramètres récurrents ${ }^{10}$ qui les distinguent des autres sites proches. Au nombre de ces critères, l'héliotropisme et l'hygrophilie sont vraiment primordiaux. Le premier signifie l'orientation méridionale des sites peints ou gravés, au sens large du terme, c'est-à-dire entre sud-ouest et sud-est. Quelques sites peuvent avoir une orientation occidentale ou orientale, aucun n'est ouvert au nord ${ }^{11}$. L'hygrophilie sous-entend une humidité périodique de l'abri, le plus souvent après des pluies importantes : des joints de strates suintent, des fissures drainent l'eau, de petits gours se remplissent, des stalactites et stalagmites sont réactivées, etc. Dans le cas de rochers gravés, l'eau circule au sein d'un réseau de cupules et de drains. L'importance du soleil et de l'eau dans le choix de l'espace rappelle donc celle des motifs censés représenter ces éléments. Ils sont les déterminants à la fois du site qui devient un lieu culturel et des êtres vivants qui opèrent leur transformation. Bien entendu, nous n'avons pas d'arguments pour expliquer la préférence qui est donnée à ces figures sauf à prendre à notre compte le postulat selon lequel elles sont des motifs à forte valeur expressive susceptibles de convenir au plus grand nombre de situations symboliques.

9. Hénaff 2008, p. 66

10. Qui sont le panoptisme, l'héliotropisme, la rubéfaction des parois et l'hygrophilie (Hameau 2002).

11. Â l'exception des abris peints dont les motifs sont tracés au fond de galeries. Les figures sont alors sans connexion avec une orientation cardinale particulière. 


\section{Bibliographie}

Acosta P. (1968). La pintura rupestre esquematica en España, Universidad de Salamanca, $250 \mathrm{p}$.

BOAS F. (2003) [1927]. L'art primitif, Paris, Adam Bird, 416 p.

Bosh J. et Estrada A. (1994). La Venus de Gavà, in J. Bosch et A. EstradA (dir.), El Neolític Postcardial a les Mines Prehistòriques de Gavà (Baix Llobregat), Rubricatum, nº, p. 287291.

BOURDIEU P. (1982). Les rites comme actes d'institution, Actes de la recherche en Sciences sociales, vol. 43, Éditions Fayard, Paris, p. 58-63.

BUENO RAMIREZ P. et BALBIN BEHRMANN R. de (2000). Art mégalithique et art de plein air. Approches de la définition du territoire pour les groupes producteurs de la Péninsule Ibérique, L'Anthropologie, t.104, p.427-458.

BREUIL H. (1933-35). Les peintures schématiques de la Péninsule Ibérique, Paris, Imprimerie Lagny, 4 vol.

Coquet M. (2001). L'anthropologie de l'art, in M. SEGALEN (dir.) Ethnologie, Concepts et aires culturelles, Armand Colin, Paris, p.140-153.

DéLÉAge P. (2013). Inventer l'écriture, Éditions des Belles Lettres, Paris, 248 p.

ELIADE M. (1965). Le sacré et le profane, Éditions Gallimard, Paris, 186 p.

GAGNIÈRE S. et GRANIER J. (1976). Catalogue raisonné des stèles anthropomorphes chalcolithiques du Musée Calvet d'Avignon, Avignon, Éditions du Musée Calvet, 75 p.

HAMEAu Ph. (1997). Les peintures schématiques de Baume Peinte (Saint-Saturnin-lès-Apt, France), Zephyrus, n50, p.179-197.

HAMEAU Ph. (2000). Implantation, organisation et évolution d'un sanctuaire préhistorique: la haute vallée du Carami (Mazaugues et Tourves, Var), $7^{\text {ème }}$ Supplément au Cahier de l'ASER (Association de Sauvegarde, d'Étude et de Recherche pour le patrimoine naturel et culturel du Centre-Var), $227 \mathrm{p}$.

HAMEAU Ph. (2001) L'art schématique linéaire dans le Sud-Est de la France, L'Anthropologie, t.105, p. 565-610.

Hameau Ph. (2 002). Passage, transformation et art schématique: l'exemple des peintures néolithiques du sud de la France, Archaeopress, Oxford, British Archaeological Reports, vol. $1044,280 \mathrm{p}$.

HAMEAU Ph. (2003). Que l'idole est antérieure à l'homme ..., Revue du Centre archéologique du Var, p. 35-42.

HAMEAU Ph. (2006). Animal et expression schématique néolithique dans le sud de la France: entre réel et idéel, in Ph. HAMEAU (éd.) Animaux peints et gravés : de la forme au signe. Le bestiaire dans les expressions graphiques post-paléolithiques en Méditerranée occidentale, Actes du Colloque international Nice juillet 2005, Anthropolozoogica, t. 41.2, p. 103-124.

HAMEAU Ph. (2009). Site, support, signe: une cohérence de sens. L'expression graphique picturale au Néolithique, in VIALOU D. (dir.) Représentations préhistoriques, images du sens, L'Anthropologie, vol. 5.3/3, p. 885-912. 
HAMEAU Ph. (2009). Expérimenter la peinture néolithique, Actes du Colloque Archéologie expérimentale, Baux-de-Provence 10 octobre 2009, Les Baux-de-Provence, Éditions du Musée d'Art et d'Archéologie des Baux-de-Provence, p. 5-14.

HAMEAU Ph. (2010a). Un passage obligé par les abris peints au Néolithique, in HAMEAU Ph., ABRY Ch. et LeTOUBlON F. (coord.) 2010, Les Rites de passage - 1909-2009 - De la Grèce d'Homère à notre XXI siècle, Le Monde alpin et rhodanien, Musée Dauphinois, Grenoble, p. 47-56.

HAMEAu Ph. (2010b). Peintures et gravures schématiques à la Bergerie des Maigres - La longue tradition graphique, ERAUL, $\mathrm{n}^{\circ} 122$, Université de Liège, $106 \mathrm{p}$.

HAMEAU Ph. (2012). Geste graphique et technicité : l'exemple des peintures néolithiques, in GaUCher Ch., CANDAu J. et Halloy A. (dir.) Anthropologie du geste, Anthropologie et Sociétés, vol. 36-3, p. 57-76.

HAMEAU Ph. (2013). Personnages masculins, signes anthropomorphes et expression graphique schématique au Néolithique dans le sud de la France, $L^{\prime}$ Anthropologie, n¹1, p. 238-255.

HAMEAU Ph. (2015). Gabriel RODRIGUEZ et Henri MARCHESI (dir.), Statues-menhirs et pierres levées du Néolithique à aujourd'hui, Les versions peintes et gravées des figures de l'expression mégalithique, Éditions de la Direction régionale des affaires culturelles Languedoc-Roussillon et Groupe Archéologique du Saint-Ponais, Actes du III Colloque de Saint-Pons-les-Thomières sur la statuaire mégalithique, p. 423-432, 2012.

HARRIS R. (1993). La Sémiologie de l'écriture, CNRS Éditions, Paris, 377 p.

HÉNAfF M. (2008). Claude Lévi-Strauss, le passeur de sens, collection Tempus, Éditions Perrin, Paris, $233 \mathrm{p}$.

LÉVI-SRAuSS Cl. (1958). L'Anthropologie structurale, Éditions Plon, Paris, 452p.

MARTineZ Garcia J. (1984). El Penon de la Virgen : un conjunto de pinturas rupestres en Gilma (Nacimiento, Almeria). Associaciones recurrentes, simbolismo y modelo de distribucion, Quadernos de Prehistoria de la Universidad de Granada, nº9, p. 39-80.

SANCHIDRIÁN TORTI J.L. et MUÑOZ VIVAS V.E. (1983). Cuestiones sobre las manifestaciones parietales post-paleoliticas en la cueva de la Pileta (Benaoján, Málaga), Zéphyrus, p. 151-163.

VAN GENNEP A. (1909). Les rites de passage, Éditions Nourry, Paris, 288 p.

VIALOU D. (1987). L'art des grottes en Ariège magdalénienne, XXII Supplément à Gallia Préhistoire, Éditions du CNRS, Paris, 432 p.

WeIss M.- Cl. (2003). L'art rupestre de la Corse, Éditions Albiana, Ajaccio, 244 p. 


\section{Illustrations}

Figure 1 : Évolution des versions graphiques du personnage masculin depuis les versions identifiables jusqu'aux versions très simplifiées : a-f, personnages complets dont un qui s'appuie sur une canne (a) et un autre qui est coiffé de plumes et est armé d'un arc (b) ; f $\mathrm{h}+\mathrm{l}$ et $\mathrm{m}$, personnages et signes anthropomorphes masculins cruciformes; $\mathrm{i}-\mathrm{k}$, signes anthropomorphes à bras ou jambes en «phi »; $n$ - p, versions arboriformes ; $q$ et $r$, signes anthropomorphes en trait vertical à extrémité supérieure renflée ou non. (DAO Ph. Hameau)

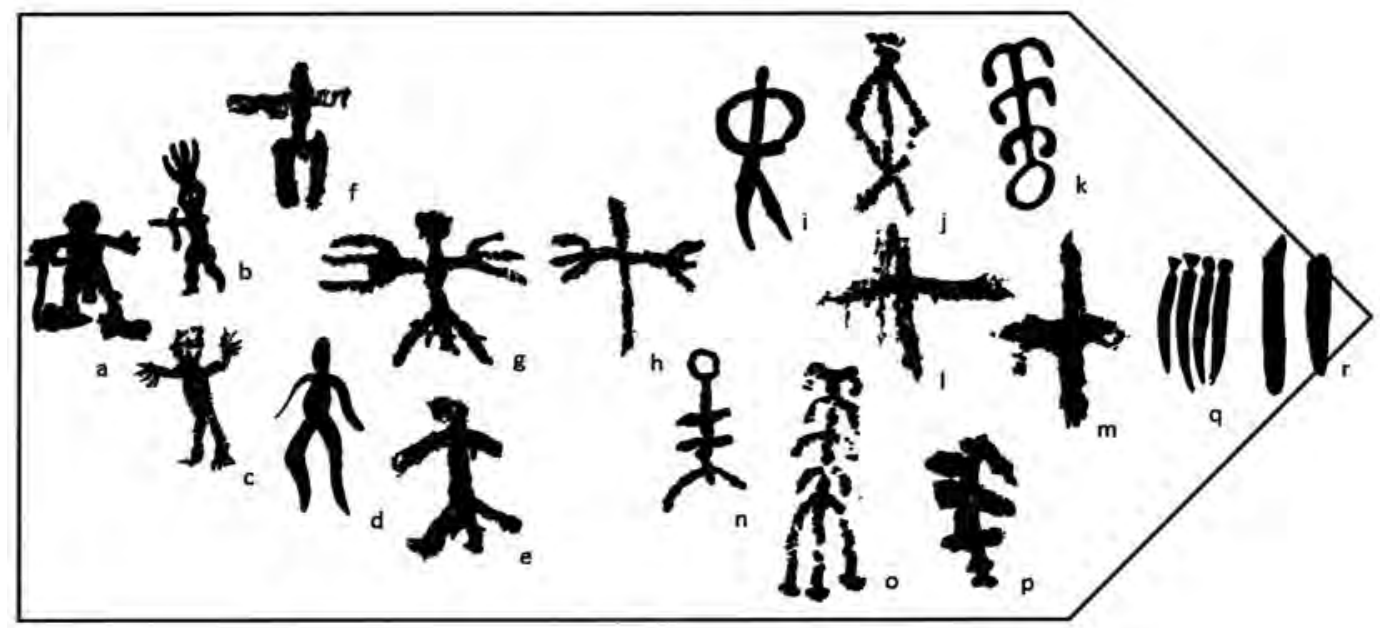

Figure 2: Les cinq principales catégories de motif de l'expression graphique du Néolithique : versions identifiables et simplifiées. (DAO Ph. Hameau)

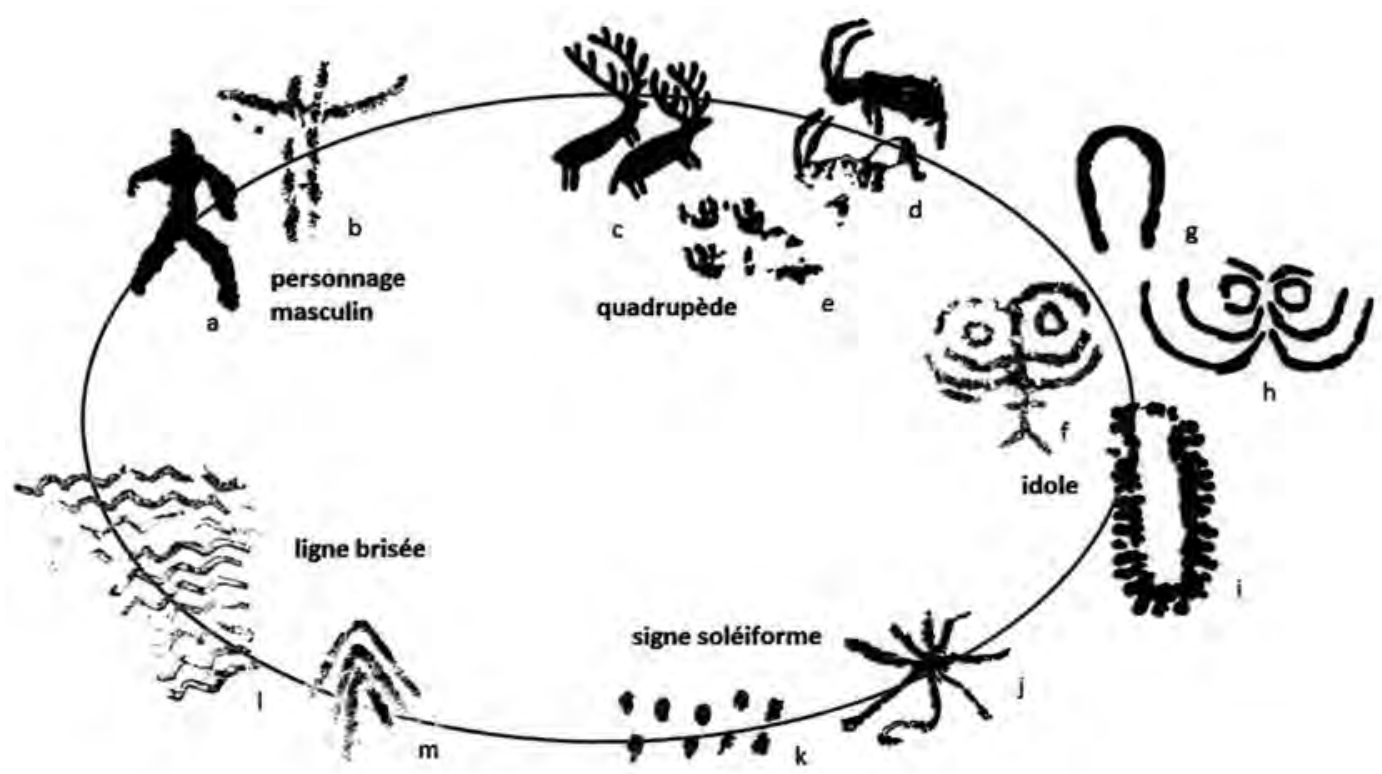


Figure 3 : Le doublement imparfait de la figure des êtres vivants : a - c, le personnage masculin, exemples en peinture; $d$ - f, l'idole, versions gravées relevées sur les pentes du Signal de la Lichère (Gard). (DAO Ph. Hameau)

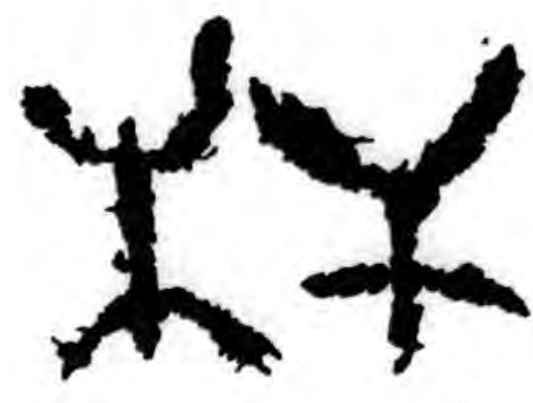

a

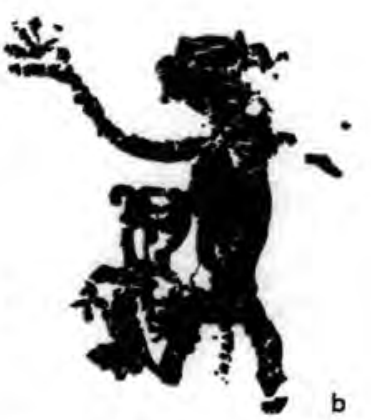

différence de taille

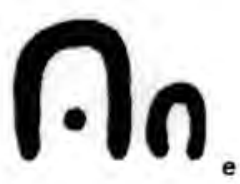

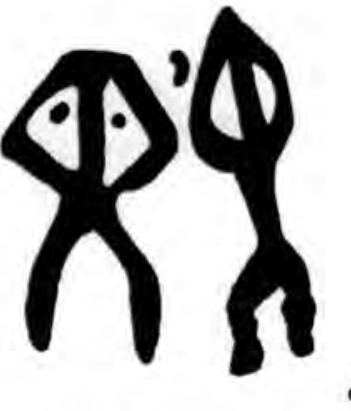

un seul des deux motifs est "ponctué "
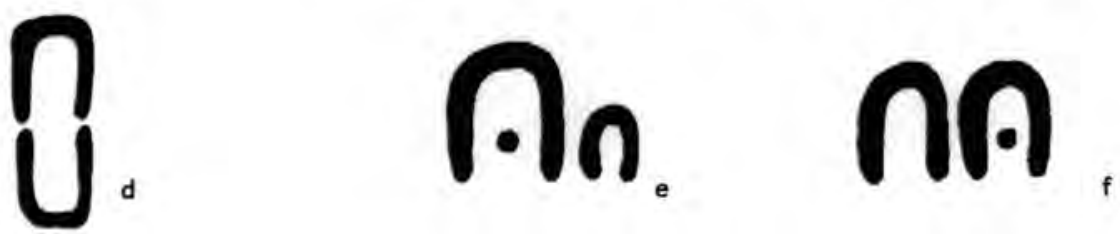

Fig. 5 : Présence du signe soléiforme et de la ligne brisée dans l'expression schématique linéaire : a, motif hémi-arboriforme dont une branche est poursuivie par une ligne brisée ; b et $c$, personnages masculins à tête ou yeux solaires flanqués d'une ligne brisée ; d, deux personnages dont la silhouette est traitée en ligne brisée; e, personnage peint au Néolithique sur lequel on a rajouté un motif gravé soléiforme. (DAO Ph. Hameau)

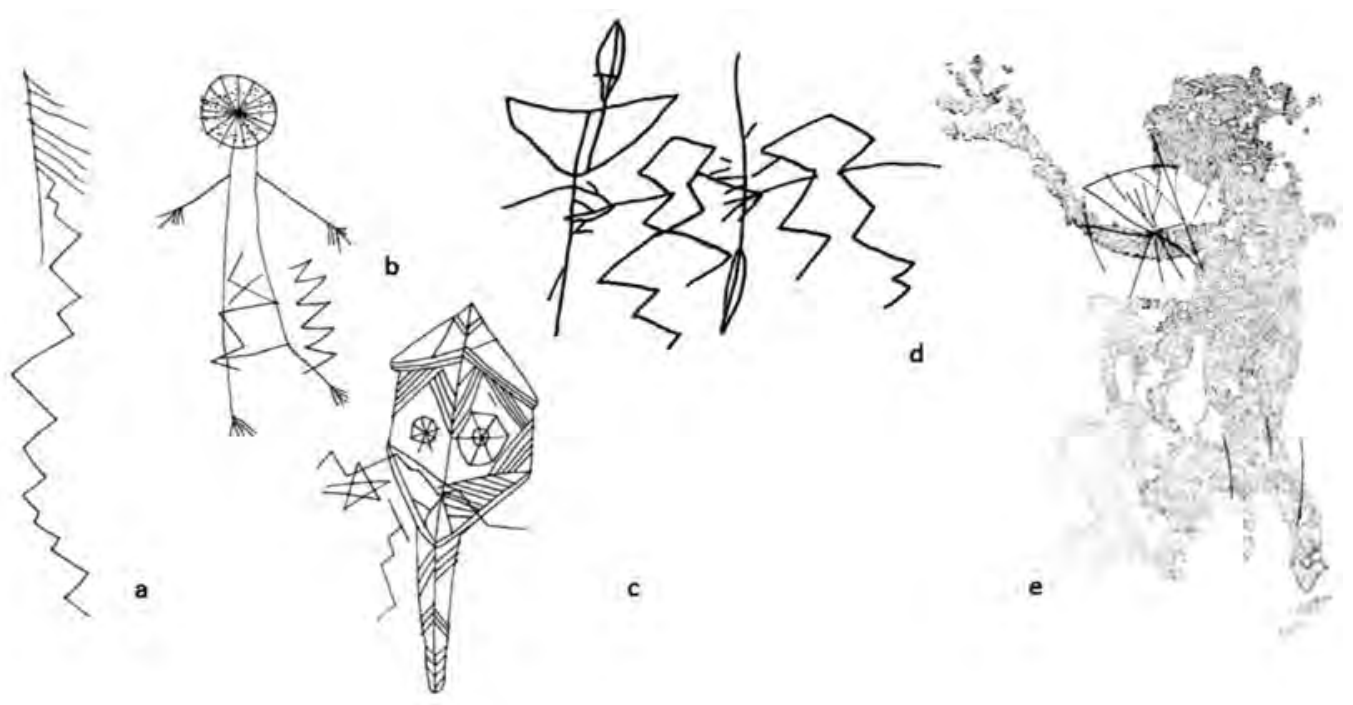




\title{
Faire " parler » les pierres \\ Le modèle naturaliste en archéologie préhistorique Le cas de la Vallée des Merveilles, 1868-1913 ${ }^{1}$
}

\author{
Maddalena CATALDI \\ Doctorante Centre Alexandre Koyré, EHESS

\begin{abstract}
$\overline{\text { Extrait de : Olivier BUCHSENSCHUTZ, Christian JEUNESSE, Claude MORDANT et Denis VIALOU (dir.), }}$ Signes et communication dans les civilisations de la parole, Paris, Édition électronique du CTHS (Actes des congrès des sociétés historiques et scientifiques), 2016.

Cet article a été validé par le comité de lecture des Éditions du CTHS dans le cadre de la publication des actes du $139^{\circ}$ Congrès national des sociétés historiques et scientifiques tenu à Nîmes en 2014.
\end{abstract}

\begin{abstract}
Résumé
Au milieu du XIX $X^{e}$ siècle, les gravures du site de la vallée des Merveilles, connues depuis le $\mathrm{XVII}^{\mathrm{e}}$ siècle, sont interprétées comme des vestiges préhistoriques par la communauté scientifique émergente des préhistoriens. Cette contribution explore la relation entre la construction du savoir sur l'Homme préhistorique et la construction des outils conceptuels susceptibles de permettre la connaissance de ses cultures. Parmi ces outils, les types sont des ensembles d'objets, rassemblés en raison de leur proximité morphologique. Déjà utilisés par l'archéologie classique, ils sont rapprochés des taxons utilisés par les sciences naturelles (espèces, genres, etc.) dans le discours de l'archéologie préhistorique en devenir.

L'article retrace la construction de ces types et leur fonction dans le discours des archéologues travaillant à la Vallée des Merveilles entre 1868 et 1913.
\end{abstract}

Mots-clés: Mont Bégo, vallée des Merveilles, gravures, épistémologie et naissance de la préhistoire, histoire des sciences et des techniques

\begin{abstract}
In the middle of the 19th century, the engravings of the vallée des Merveilles, known of since the 17the century, were dated to Prehistory by the emerging scientific community. This paper will explore the relationship between the growing knowledge of Prehistoric Man and the elaboration of the concepts used in study of prehistoric cultures. One of these tools is the "type" defined as a group of objects with similar morphologies. Already used in classical archaeology, the "type" used in this emerging prehistoric archaeology was based on the taxons of the Natural Sciences (species, genus, etc).

This paper retraces the construction of these types and their use in the archaeological research of the Vallée des Merveilles between 1868 and 1913. (traduction Rebecca Peake)
\end{abstract}

Le site archéologique de la Vallée des Merveilles (Alpes-Maritimes, France) préserve près de 40000 figures, datées du Chalcolithique jusqu'à l'âge du Bronze, gravées sur les rochers des vallées qui entourent le Mont Bego $(2872 \mathrm{~m})^{2}$.

1. Je voudrais remercier Laurent Puymerail et Patricia Wils pour avoir si patiemment et toujours généreusement corrigé mon français, Volny Fages pour sa relecture attentive et son soutien, Florent Detroit pour l'inspiration. 2. Je fais référence ici aux études actuelles sur le Mont Bego, produites par l'équipe du Pr. Henry de Lumley, en particulier parmi les plus récentes : de Lumley H. et al. 2011. 
Ces gravures, connues depuis le $\mathrm{XVII}^{\mathrm{e}}$ siècle, notamment dans des chorographies (Gioffredo 1839) et des statistiques descriptives (Fodéré 1821) ${ }^{3}$, sont, dans la seconde moitié du XIX $X^{\mathrm{e}}$ siècle, interprétées comme les vestiges des peuples préhistoriques ayant occupé la région. Cette interprétation se construit dans le discours de la discipline préhistorique au moment même où celle-ci est en train d'émerger comme science. En effet, ces gravures sont présentées pour la première fois dans une des toutes premières réunions internationales spécifiquement instituées " pour les études préhistoriques " ${ }^{4}:$ le troisième congrès d'Archéologie préhistorique à Norwich (Angleterre) en 1868 (Moggridge M. 1869).

À partir de ce moment fondateur des études scientifiques sur les cultures de l'Homme préhistorique, ces figures ont donné lieu à une grande variété d'interprétations, parfois contradictoires. Comment comprendre, rétrospectivement, la diversité des attributions de ces mêmes figures? Comment cet épisode peut-il éclairer la phase initiale de la formalisation des concepts et des pratiques des préhistoriens?

À l'époque, ces derniers appuient leurs interprétations - les attributions à un peuple ou l'interprétation de la fonction de ces représentations dans les cultures primitives - sur ce qu'ils appellent la "détermination ", c'est-à-dire le procédé qui conduit à nommer une représentation ou un type, un ensemble d'objets, rassemblés en raison de leur proximité morphologique. L'origine de ce procédé, qu'il vienne de l'archéologie classique ou d'un modèle plutôt naturaliste, a fait l'objet de débats depuis (Schlanger 2010, Gräslund 1987). $\mathrm{Au}$ travers de l'étude du cas de la Vallée des Merveilles, il s'agira de considérer la construction de types dans la perspective d'une histoire de la constitution de la discipline préhistorique. L'exemple des classifications taxonomiques forgées à partir de ces gravures, montre que l'acquisition des connaissances et la construction de la discipline ne peuvent pas être séparées dans l'analyse historiographique.

Dans cet article, sont présentés les trois moments de la trajectoire qui conduit de l'émergence des gravures de la Vallée des Merveilles dans le discours scientifique sur les cultures préhistoriques, à la construction d'un modèle consensuel pour la « détermination » de ces gravures. Sera analysée également l'évolution de la diffusion des gravures de la Vallée des Merveilles, en particulier au sein des lieux de présentation et de conservation scientifique (congrès et musées), ainsi que les pratiques qui leur sont associées, pour comprendre comment les savants composent les types à la base de leur discours.

\section{Matthew Moggridge, de la Vallée des Merveilles à Norwich}

Matthew Moggridge (1803-1882) et son fils John (1842-1874) font partie de la diaspora de notables anglais qui investit la Riviera, en Italie et en France, tout au long du XIX siècle (Dawes 2003). Ils s'installent à Menton pour des raisons de santé, afin que John puisse profiter des bienfaits du climat du Sud (Moggridge J.T. 1866). Les deux sont botanistes, associés à la Linnean Society de Londres; le père travaille sur les conifères des côtes méditerranéennes (Moggridge M. 1867), le fils publie plusieurs observations sur les orchidées du genre Ophrys et une flore de Menton (Moggridge J.T. 1866, 1867, 1871). Matthew Moggridge est par ailleurs membre de la Geological Society de Londres. À l'été 1867, accompagné par un naturaliste prussien, probablement Georg Dieck (1847-1925), M. Moggridge part en direction du Mont Bego afin de reproduire les gravures des lacs des Merveilles. Les deux savants sont équipés pour faire des moulages et des estampages mais, à cause du mauvais temps qui approche et de la difficulté du travail en plein air à

3. Cette publication est basée sur une Statistique des Alpes Maritimes, rédigée en 1803 et non publiée.

4. En français et en italique dans le texte. «Origin and Designation of the Congress», p. XIV. 
cette altitude, G. Dieck finira par réaliser des dessins à l'œil, pendant que M. Moggridge explore la vallée et lui indique les gravures à relever. C'est la première fois que l'on reproduit les gravures de la Vallée des Merveilles pour publication (fig. 1). Même si les autres communautés épistémiques que l'on a évoquées avaient connaissance de leur existence et qu'elles avaient donné des interprétations sur l'origine de ces figures, en attribuant leur production à l'armée d'Hannibal, ou à des bergers ${ }^{5}$, la reproduction et la diffusion des figures n'étaient pas nécessaires à leur analyse. Par contre, pour la communauté des archéologues émergeant à cette époque, la production des figures par copie fait partie du travail que l'archéologue se doit d'accomplir. Ainsi, il est intéressant ici de remarquer que $M$. Moggridge, dans sa communication, ne s'aventure pas à identifier les auteurs des gravures observées dans la Vallée des Merveilles, mais il propose une lecture des gravures suggérant une analogie avec les hiéroglyphes, une même figure se reproduisant en combinaisons variées.

Afin de saisir la nature et l'importance de l'obtention et de la publication de gravures de spécimens pour la communauté émergente des archéologues préhistoriens, il est essentiel d'analyser de plus près les procédés et les pratiques qui y sont alors associées.

Cette pratique peut être comparée à celle des naturalistes. Bruno Latour a décrit ce processus dans son article sur Boa Vista (Latour 1993) en indiquant les étapes qui amènent les objets naturels, de leur contexte (la nature), à une conceptualisation et donc au débat scientifique. Comme une plante, détachée de son milieu local (une forêt, une prairie...), peut être enfin conservée et classée dans un herbier, les gravures de la Vallée des Merveilles, détachées de leurs rochers par copies, et séparées des autres gravures présentes sur la roche, deviennent des figures sur papier, qui peuvent désormais être conservées, reproduites et comparées. Dans le cas des botanistes, il ne s'agit pas uniquement de conserver la plante; dans la pratique, la production de planches d'aquarelles a la même importance que le recueil de spécimens. Lors des expéditions coloniales en Amérique du Sud, comme l'a décrit par exemple Daniela Bleichmar pour l'empire espagnol, les botanistes des Lumières se font accompagner sur le terrain par des artistes et dirigent de près leurs choix et la production d'aquarelles qui formeront les flores locales destinées à être connues et étudiées en Europe. Faire circuler ces « faits naturels ", une fois copiés sur une page blanche qui les détache de l'environnement d'où ils sont tirés, est une qualité de base requise pour pouvoir ensuite les ré-agréger dans un système naturel, celui de Linné (Bleichmar 2009).

Dans la Vallée des Merveilles, le naturaliste G. Dieck, qui accompagne M. Moggridge, dessine les gravures choisies par ce dernier et qui vont être publiées dans les actes du congrès de Norwich et faire leur entrée dans le discours sur les peuples primitifs. Hodder Micheal Westropp (1820-1885), archéologue irlandais, insère les gravures de la Vallée des Merveilles, qu'il a vues à Norwich et donc qu'il peut comparer avec d'autres, dans sa publication de synthèse sur la pratique de la gravure dans les cultures primitives, publiée dans les Actes du congrès (Westropp 1869). Les gravures des Merveilles sont prêtes donc pour circuler dans la communauté émergente des préhistoriens et elles peuvent être comparées avec d'autres, provenant d'autres sites.

Chez M. Moggridge, les gravures sont dessinées et reproduites sans commentaires. Les figures sont divisées en cinq planches pour la publication où elles se présentent de façon dispersée sur la page, sans numéros qui les renverraient au texte et sans ordre explicité. M. Moggridge fait des choix et il ne prend pas en compte l'unité de la roche. Il considère toutes les gravures séparément, mais il ne construit pas des types: il traite chaque gravure comme une singularité, puisqu'il reproduit exclusivement les figures qui ressemblent à d'autres déjà connues, rencontrées dans des musées ou des publications. Il fonde ainsi son raisonnement et sa présentation graphique sur une analogie

5. Il s'agit des attributions faites par F.E. Fodéré, dans sa Statistique des Alpes Maritimes en 1803 et dans la Corografia delle Alpi Marittime de $1690 \mathrm{ca}$ de Pietro Gioffredo. 
décontextualisée, tant d'un point de vue spatial que temporel. Il identifie ainsi une « idole sarde » (Moggridge M. 1869, p. 361), vue dans un musée à Turin, ou des gravures ressemblant aux cornes des antilopes. On peut dire que M. Moggridge agit comme un naturaliste dans sa pratique (la copie des gravures), mais qu'il n'organise pas ses gravures de cette façon, puisqu'il les considère comme des figures singulières, chacune avec sa signification spécifique. M. Moggridge possède une méthode naturaliste, mais il ne va pas créer un système naturaliste.

\section{Une controverse française}

Sous leur forme imprimée, les gravures fournissent alors matière à controverses entre préhistoriens. Autour de 1877, la Vallée des Merveilles fait son apparition sur la scène française, peu de temps après la traduction de l'article de M. Moggridge en 1875 dans la Revue Archéologique, dans laquelle les figures sont groupées en deux planches (Moggridge M. 1875). Plusieurs articles paraissant en France, l'intérêt est porté ici plus spécifiquement sur trois d'entre eux, ceux qui reproduisent les gravures.

Léon Clugnet (1848 - après 1905), à cette époque conservateur de la bibliothèque de Lyon, explore la Vallée des Merveilles pendant deux jours, à la fin du mois d'août 1877. L. Clugnet dessine à main levée près de 150 figures, dont la revue des préhistoriens et anthropologues matérialistes "Matériaux pour l'histoire primitive et naturelle de l'Homme » présente une sélection (Clugnet 1877, p. 379-387) (fig. 2). Pour L. Clugnet, comme pour M. Moggridge, l'origine des gravures demeure incertaine, mais elles ont vraisemblablement été réalisées par des bergers du fait qu'on trouve représentés les outils et les animaux qui peuvent correspondre à une vie pastorale, dont la monotonie est exprimée dans la répétition des figurations et dans l'absence de «tableaux historiques représentant des événements remarquables » (ibid. p. 385). Ceci traduit donc une société vivant aux marges de l'histoire, celle des bergers.

Cette attribution s'accorde avec une datation avant l'âge du Métal, qu'il propose en s'appuyant sur la forme massive des armes représentées qui sont aussi celles qui ont servi pour réaliser les gravures. Elles ne peuvent donc pas être en métal, puisqu'elles se seraient cassées au contact avec les rochers. Pour étayer son argumentation, il va au Musée de Saint-Germain-en-Laye et prouve par la pratique que des outils en pierre seraient plus convenables pour graver sur ce type de rochers.

L. Clugnet divise les gravures en groupes finement différenciés. Par exemple, il fait la différence entre les bovidés (bovinés et caprinés) avec des «têtes de bœuf » et des " têtes de béliers, boucs, bouquetins " ou alors il repère les outils plus conformes à la vie pastorale comme des "filets servant à transporter le foin dont on se sert encore dans quelques hautes vallées des Alpes » (ibid. p. 386). Il détermine également des figures singulières, par exemple un chien de berger, un oiseau. Dans les planches publiées dans Matériaux, les figures, sans ordre évident sur la page, sont numérotées afin d'être mises en relation avec la liste de groupes dans le texte. L. Clugnet crée des types, c'est-à-dire des ensembles de figures qui se ressemblent, mais il les détermine de façon à les singulariser; il n'hésite d'ailleurs pas à créer des groupes avec des figures uniques. Son système prête à contestation sur ce point. Les figures singulières ne vont pas être " déterminées » de la même façon par d'autres préhistoriens et elles fragilisent donc la stabilité de son système. En revanche, la pratique «naturaliste» des copies de transformation des gravures en figures devient centrale, au point qu'elle sera la base des attaques de E. Rivière, qui l'accusera du vol de ses copies, et donc de lui avoir soustrait sa « découverte».

En effet, L. Clugnet et E. Rivière se croisent sur le terrain en descendant dans la vallée. 
Émile Rivière (1835-1922) qui résidait dans la région pour soigner sa faible santé, avait entrepris, dès 1872, des fouilles aux Baoussé-Roussé, des grottes dans les environs de Menton. Ces travaux, financés par le Ministère de l'Instruction Publique, avaient livré un ensemble de squelettes appartenant à la « race de Cro-Magnon », couchés et aux crânes ornés de coquilles et d'ocre. Cette découverte exceptionnelle lui avait valu une certaine renommée et il avait été nommé, entre autres, membre correspondant de la Société des Sciences naturelles, des Lettres et des Beaux-arts de Cannes et de l'arrondissement de Grasse en 1873. Au musée de cette Société, il avait vu les estampages des gravures des Merveilles offerts par le docteur Battersby en $1868^{6}$. Ayant obtenu un financement du Ministère pour sa nouvelle mission aux lacs des Merveilles, E. Rivière et son adjoint, l'architecte et archéologue Léon de Vesly (1844-1920) partent en 1877 pour l'Italie, avec une chambre claire pour copier les figures et un théodolite pour dessiner une carte de la vallée ${ }^{7}$. Le but, affiché dans la demande de financement au Ministère, est de réaliser des estampages, une technique de copie plus fidèle que le dessin, qui aurait évité "les erreurs » présentes dans les dessins de $\mathrm{M}$. Moggridge, défini d'ailleurs comme "plus naturaliste qu' archéologue $»^{8}$.

Le rapport de mission est manquant dans le dossier conservé aux Archives nationales, mais on sait que, pendant 10 jours, E. Rivière et L. de Vesly ont pu effectuer 400 estampages, un album de dessins des roches, réalisé par L. de Vesly et une carte topographique de la région des gravures. Ils n'ont pas pu se servir de la chambre claire, saisie par les militaires italiens au début de leur mission'. Alexandre Bertrand (18201902), archéologue et membre de l'Académie des Inscriptions et Belles Lettres, directeur du Musée d'Archéologie Nationale (MAN) de Saint-Germain-en-Laye depuis son ouverture en 1868, interrogé par le Ministère sur l'importance des résultats de la mission, est mécontent du rapport, jugeant les 19 premières pages, sur 39, anecdotiques ${ }^{10}$. Pour G. Bertrand, formé à l'archéologie classique et spécialiste des vestiges gaulois, les résultats de la mission sont également décevants. Il avance qu'on aurait pu éventuellement faire mouler un des rochers, choisi sur la base des dessins de L. de Vesly, pour l'exposer au MAN et faire circuler des copies des types les plus représentatifs des gravures des Merveilles $^{11}$. Le rapport de G. Bertrand est intéressant parce qu'il nous permet de remarquer que, pour lui, la roche présente un intérêt dans son ensemble, comme objet d'étude et qu'elle est digne de conservation.

Nonobstant l'avis de G. Bertrand, opposé à la publication, E. Rivière publie une partie des gravures dans les actes du congrès de l'Association française pour l'Avancement des Sciences (AFAS), suite à leur présentation au congrès de cette association à Paris en 1878 (Rivière 1879). Nombre de ces estampages sont d'ailleurs exposés par le Ministère pendant l'Exposition Universelle de Paris de cette même année.

Les figures relevées sont regroupées par E. Rivière en trois groupes selon les objets représentés: des «animaux», des «armes, instruments et outils», des «signes indéterminables, mais se rapportant à un type à peu près toujours le même ", et publiées sur une seule planche (fig. 3) (ibid., p. 787). Pour la publication, E. Rivière a copié au pantographe les contours des estampages pour les réduire au sixième de leur grandeur naturelle. Les figures sont identifiées individuellement sur la planche par un numéro qui fait référence à sa détermination donnée dans le texte. En effet, E. Rivière essaye d'identifier chaque figure et par exemple, dans les «signes indéterminables », qu'il dit tous apparentés à un type à peu près identique, l'auteur propose de considérer certains

6. Arch. nat. Ministère de l'Instruction publique , F 173003 A, fol. 1. Cfr. « Dons faits aux Musée », p. 117.

7. Arch. nat. Ministère de l'Instruction publique, F 173003 A, « Note des objets saisis, extrait du procès-verbal dressé par M. le Capitain Somale, en date 1 juillet 1877 ».

8. Arch. nat. Ministère de l'Instruction publique , F 173003 A, fol. 1.

9. Arch. nat. Ministère de l'Instruction publique, F 173003 A, fol. 33

10. Arch. nat. Ministère de l'Instruction publique, F 173003 A, fol. 49. Il s'agit de neuf pages d'historique des recherches sur le site et dix pages portant sur les difficultés de la mission. Il s'agit probablement du rapport de l'expulsion par les autorités italiennes, méfiantes à l'égard d'étrangers dessinant des cartes de la zone de frontière, au tout début de la mission, survenue en juillet.

11. Ibid. 
comme des « filets », des « galettes » ou des " gâteaux », d'autres comme des " anneaux », des "roues" ou des "rouelles", et d'autres encore comme des sortes de "manches ou timons », comme s'il s'agissait d'éléments d'un véhicule, puis d'autres enfin comme « des clôtures ou des barrières ». Elles se rapportent toutes au type affectant la forme d'un " cercle », d'un « ovale », d'un « carré » ou d'un " rectangle plus ou moins allongé » (Ibid. p. 789-790). Il est intéressant de remarquer ici que G. Bertrand, dans son rapport, avait renommé le groupe des «animaux » qu'il appelait «cornes avec frontal», ou «frontal cornu »; il considérait que le corps de l'animal n'était jamais représenté et qu'il s'agissait donc d'un unique groupe de cornus avec des variations, qu'il appelait "types », ou «variantes ${ }^{12}$. E. Riviére revient donc à l'idée de G. Bertrand : ils créent ensemble ce que l'on pourrait appeler un taxon, un groupe plus large destiné à absorber des types différents, que Riviére considère comme des variantes d'une unique représentation.

E. Rivière utilise ses copies pour les comparer avec d'autres, provenant des Canaries. Il maintient que les gravures du Mont Bego présentent « une véritable parenté » (Rivière 1879 p. 783) avec celles-là. Cette " découverte », confirmerait l'hypothèse de l'expansion des Guanches d'Afrique en Europe (ibid. p. 783). En passant par le Périgord, ces « hommes de Cro-Magnon » seraient venus s'installer à l'âge du Bronze, au Col de Tende. Cette "découverte » s'appuie sur la ressemblance des gravures des deux établissements éloignés dans le temps et dans l'espace. L'idée est que si l'on peut, par comparaison, construire une filiation entre les crânes et les restes fossiles, donc entre la même " race » habitant différents sites, on trouvera de la même façon une "parenté » entre les représentations gravées par cette « race ».

En 1878, c'est Edmond Blanc (1841-19..), archéologue et directeur de la bibliothèque municipale de Nice, correspondant du Ministère de l'Instruction publique pour les Travaux historiques, qui s'intéresse aux gravures des Merveilles. Il s'agit d'un des fondateurs de la Société des Sciences naturelles et historiques des Lettres et des BeauxArts de Cannes et de l'arrondissement de Grasse, où E. Rivière avait pu voir des estampages des gravures avant sa mission.

E. Blanc conteste l'attribution par L. Clugnet des gravures à des bergers de l'âge de la Pierre, puisque celle-ci est construite à partir du système des groupes de figures. E. Blanc écrit que L. Clugnet, en présentant les gravures, a voulu « placer ensemble les objets de la même nature, sans tenir compte de la façon dont ils sont groupés sur les rochers » (Blanc 1879, p. 73) et il propose d'interpréter ces figures, qu'il reproduit dans les Mémoires de la Société, comme des «ex-voto » pour une divinité terrible, alléguant une description épouvantable des lieux environnants. La toponomastique, qui rappelle enfer, démons et sorcières, constitue un autre argument à l'appui de sa thèse et une partie de son texte est donc dédiée à une description pittoresque des Vallées, cet élément prenant le statut d'argument pour une explication scientifique. Les planches publiées (fig. 4) sont en partie des dessins, repris au pantographe, des estampages réalisés par l'auteur, mais une partie reprend les figures publiées par L. Clugnet pour en donner une nouvelle «détermination» (ibid. p. 83). Ainsi E. Blanc identifie toutes les figures, une par une : il reconnaît des «amulettes » parmi les nouvelles figures reproduites et voit un « homme armé, brandissant de la main droite un poignard et de l'autre un objet difficile à déterminer » là où Clugnet avait vu un « oiseau » (ibid. p. 85-86).

Les archéologues français L. Clugnet, E. Rivière et E. Blanc s'accordent sur la façon de reproduire des gravures, la production de figures par copie. Cette pratique demeure dorénavant consensuelle et la production des copies est perçue comme centrale dans le travail d'un archéologue, comme le montrent les accusations de E. Rivière à L. Clugnet d'avoir copié ses estampages, pour lui voler sa "découverte », source du litige qui durera jusqu'en 1879 (Clugnet 1879, p. 235-239). Une fois les gravures réduites en figures sur papier, les archéologues peuvent les déplacer à leur gré d'un groupe à un autre, ou alors 
les comparer avec des figures issues de sites géographiquement très éloignés. Cette phase structure le discours des préhistoriens, en fournissant un terrain commun pour les échanges et la discussion. C'est ici que les archéologues font émerger des modèles, des groupes, liés par affinités morphologiques. Cette concordance de méthode est visible dans la publication des planches, analogues pour les trois auteurs: chaque figure est numérotée, le numéro renvoyant à une « détermination » dans le texte.

Par contre, différentes approches concernent la « détermination », c'est-à-dire le procédé qui conduit au groupement et donc à l'identification des gravures. L. Clugnet crée un grand nombre de types et identifie des figures singulières. E. Rivière, construit peu de groupes, mais il les compose de figures singulières; il s'oppose ainsi à l'idée à la base de l'approche de G. Bertrand qui était, par contre, pour une construction de taxons plus larges, formés par les types, considérés donc comme des variations d'une même représentation. L'approche de E. Blanc s'oppose à celle de L. Clugnet et il nomme, une par une, chaque figure dont il dispose.

\section{Clarence Bicknell et Arturo Issel}

Clarence Bicknell (1842-1918) s'installe à Bordighera (Imperia, Italie) en 1879. Ancien révérend anglican, il est né au sein d'une famille fortunée de Herne Hill, au sud de Londres. Tout comme les Moggridge, c'est une figure éminente et active de la communauté anglaise installée sur le côté italien de la Riviera. Espérantiste, il participe aux congrès européens des amateurs de cette langue utopique. Botaniste, il constitue un herbier qu'il léguera ensuite à l'Istituto botanico de l'Université de Gênes, avec plus de trois mille aquarelles, dont une partie est conservée dans ce même institut et publiée dans deux Flora dédiées à la Riviera (Bicknell 1885, 1896). En position périphérique par rapport au réseau des institutions et associations des spécialistes, il tisse cependant des relations savantes, faites d'échanges de spécimens et d'informations avec des botanistes italiens et suisses renommés, tels que Saverio Belli (1852-1919), Émile Burnat (1828-1920) et John Isaac Briquet (1870-1931).

Dans la dernière décennie du $\mathrm{XIX}^{\mathrm{e}}$ siècle, les intérêts de ce savant basculent vers l'archéologie. Il entame alors un travail de relevé des gravures de la Vallée des Merveilles et de celles de la Vallée de Fontanalba, de l'autre côté du Mont Bego. Pour mener à bien son ambitieux projet d'inventaire d'un maximum de figures gravées, il fera construire un chalet aux flancs de cette montagne, dans la Vallée de Casterino. Les gravures, copiées chaque été lors d'une campagne sur le site, sont publiées en série entre 1898 et 1913 chez différents éditeurs, en italien comme en anglais (Bicknell 1898, 1899, 1902, 1903, 1909, 1913).

C. Bicknell se considère comme botaniste et archéologue amateur et il laisse l'interprétation des gravures à Arturo Issel (1842-1922), savant géologue et paléontologue génois avec qui il est constamment en contact et qui publiera un important travail dédié aux peuples préhistoriques installés en Ligurie (Issel 1901, 1908). Le travail de C. Bicknell est conséquent. Il copie par estampage un nombre considérable de gravures, plus de 12 000. La planche reproduite en figure 5 montre l'intérêt de son organisation des figures. Les gravures ne sont pas nommées en référence à leur ressemblance à des animaux ou outils qui serait perçue par l'archéologue, mais elles sont rassemblées en un petit nombre de groupes, huit, par leurs ressemblances morphologiques. Elles sont ensuite nommées : le grand groupe des " armes », le groupe de « figures qui ont des cornes », des figures qui sont nommées "peaux ou de type peaux», des "figures géométriques" et des « attelages ».

La nouvelle organisation des figures est bien visible dans la configuration des pages de la 
dernière œuvre de C. Bicknell, A guide to the prehistoric rocks engravings in the Italian Maritime Alps de 1913, où les représentations du même type sont rassemblées sur une même planche (fig. 5). D'ailleurs, les numéros qui servaient pour identifier chaque gravure dans les publications des archéologues précédemment évoquées et qui en faisaient une entité singulière, disparaissent de la planche de $\mathrm{C}$. Bicknell. Cet agencement graphique qui fait ressortir l'unité des figures sur la planche, leur nature commune, constitue un argument montrant la pertinence de cette classification. À travers ces groupes, C. Bicknell considère les différences qui auparavant posaient des problèmes (par ex. boucs, vaches, bouquetins, etc.) comme des variations d'un objet unique («les animaux avec cornes »). Rassemblant toutes ces variations autour d'une seule idée, celle d'un animal cornu, C. Bicknell crée la catégorie, déjà suggérée par G. Bertrand et utilisée jusqu'à nos jours, de corniforme. On voit ici la création d'un taxon qui peut être désormais accepté en dehors de l'attribution à un peuple préhistorique ou à un autre.

Arturo Issel, géologue, paléontologue et minéralogiste, auteur de travaux internationalement reconnus sur la préhistoire de la zone ligure, appuie sa publication de 1901 dans la revue de référence des paléontologues italiens sur les frottis produits par C. Bicknell. Son texte reprend plusieurs figures publiées par les archéologues évoqués précédemment pour en changer la «détermination ». Les figures produites par d'autres de façon dispersée, se trouvent maintenant absorbées dans les taxons de C. Bicknell. Les figures qui restent isolées, singulières, perdent leur force dans ce système et elles se trouvent, maintenant, dans une position marginale, n'étant pas significatives de l'ensemble des gravures du site. Les groupes représentatifs réduisent l'importance des figures singulières, qui avaient été auparavant à l'origine d'affrontements entre archéologues. A. Issel propose une synthèse des attributions des gravures faites par d'autres archéologues, les reliant à différents peuples (Ibères, Phéniciens et Ligures ${ }^{13}$ ) et montrant que ces hypothèses ne sont pas radicalement différentes. Il allègue le fait que les différents peuples de l'âge des Métaux sont, en effet, tous liés par leur appartenance à une même « race », idée déjà à la base de l'hypothèse de E. Rivière (Issel 1901, p. 251-253). Les peuples sont donc des variations au sein de la même "race ", celle de Cro-Magnon. Cette idée est inséparable du modèle de C. Bicknell, qui considère les différences morphologiques entre représentations comme des variations au sein d'un même taxon.

La trajectoire de la formation des types de la Vallée des Merveilles montre que la construction de ce modèle de classification structurant l'archéologie préhistorique comme discipline, n'est pas anodine. Si les savants qui y participent ont plutôt des formations de naturalistes, on voit aussi l'influence de l'archéologie classique. Par exemple, G. Bertrand, archéologue classique, suggère en premier et explicitement un modèle basé sur des groupes larges, des taxons formés de «variations» d'une même forme. Mais cette démarche n'est pas consensuelle pour les archéologues classiques non plus, comme le démontre l'opposition de E. Blanc à L. Clugnet sur ce point.

En revanche, le modèle des taxons, plus larges et moins discriminants, formés par variations morphologiques qui groupent finalement les types, permet de créer des liaisons entre sites de peuplements non contemporains, des moments successifs de l'histoire naturelle - de la race de Cro-Magnon, dans les propositions de E. Rivière, d'Issel et Bicknell -; bref, il s'agit d'indexer à une "race» ces représentations qui peuvent maintenant être placées dans une chronologie. Ceci est d'autant plus important dans le cas des représentations culturelles, que les gravures ne peuvent pas être mises en succession par le biais de la stratigraphie comme on peut le faire avec l'industrie lithique par exemple. D'ailleurs, dans un article de 1878, l'anthropologue Arthur Bordier pourra relier, sur cette base, les gravures des Merveilles de l'âge du Bronze et celles de ses contemporains Boschimans d'Afrique du sud (Bordier 1878).

13. A. Issel fait référence aux travaux de synthèse d'archéologues allemands, italiens et français non évoqués ici. Il s'agit d'auteurs, sauf E. Rivière, qui ne produisent pas leurs propres figures. Pour cette raison, ils ne sont pas pris en compte dans cette étude. 
La nouveauté de cette démarche est soulignée dans un texte qui théorise l'utilisation de ces catégories naturalistes en archéologie préhistorique. Il s'agit de Typology and the Theory of Evolution applied to Human Labour (1899), de l'archéologue suédois Oscar Montelius (1843-1921), spécialiste de la chronologie de l'âge des Métaux. O. Montelius maintient que les archéologues ont une façon de procéder proche de celle des naturalistes, puisqu'ils classent leurs objets collectés de manière à ce que the results are immediately obvious (les résultats soient immédiatement visibles) par proximité morphologique (Gräslund 1987, p. 103). Comme son maître, Hans Hildebrand (18421913), O. Montelius est très actif dans les congrès préhistoriques internationaux ; les deux s'investissent dans une campagne pour rapprocher les archéologues des naturalistes. Ils voudraient faire une place à l'archéologie préhistorique dans le champ des sciences naturelles.

Les deux sont bien conscients que les types sont utilisés par l'archéologie classique et notamment par la numismatique (ibid. p. 98). Mais si Montelius et son maître revendiquent une formation naturaliste pour les préhistoriens, c'est justement parce que les types dont ils doivent se servir ne sont pas ceux qu'utilise l'archéologie classique. Les nouveaux types des préhistoriens sont explicitement comparés par ces auteurs aux espèces définies par les naturalistes. Comme les espèces du monde organique se suivent dans la chaîne évolutive, les types représentent des étapes dans l'évolution culturelle humaine (ibid., p. 102). Ils ne servent plus uniquement à comparer les antiquités des différents peuples, comme le fait l'archéologue classique.

Maintenant, la tâche du préhistorien est :

« To trace the international connection between the types and to show how one is developed from the other. We call this typology (... ) (A) method which, in actual fact, is nothing else than that of the natural scientist, although it is applied not to the productions of nature but to relics of man's prehistory. $)^{14}$

Hildebrand et Montelius considèrent les types comme des catégories nécessaires pour le discours naturaliste de cette communauté scientifique émergente. Quelle que soit leur origine disciplinaire, ils agissent comme des types naturalistes et, si ce sont des concepts pivots entre l'archéologie classique et la préhistoire, ils sont appellés, par les fondateurs de cette dernière, à justifier la possibilité d'un discours scientifique sur les cultures humaines.

14. La tâche du préhistorien est « de tracer la connexion internationale entre les types et de montrer comment ils se développent les uns à partir des autres. On appelle ce procédé "typologie". Il s'agit d'une méthode qui ne diffère point, dans les faits, de celle des naturalistes, même si elle est appliquée non pas aux objets naturels mais aux ruines de la préhistoire humaine » (traduction de l'auteur) (cité par Gräslund 1987, p. 103). 


\section{Bibliographie}

Anonyme (1869a). Dons faits au Musée, Mémoires de la Société des Sciences naturelles et historiques des Lettres et des Beaux-arts de Cannes et de l'arrondissement de Grasse. vol. 1, p. 117.

Anonyme (1869b). Origin and Designation of the Congress, International Congress of Prehistoric Archaeology, London, Longmans, Green and $\mathrm{C}^{\circ}$, p. XIV-XV.

BICKNELL C. (1885). Flowering plants and ferns of Riviera and the neighbouring mountains, London, Trübner and $\mathrm{C}^{\circ}$.

BICKNELL C. (1896). Flora of Bordighera and San Remo, Bordighera, Gibelli.

BICKNELL C. (1898). Le figure incise sulle rocce in Val Fontanalba, Genova, Ciminago.

BICKNELl C. (1899). Osservazioni ulteriori sulle incisioni rupestri in Val Fontanalba, Genova, Ciminago.

BICKNELL C. (1902). The prehistoric rocks engravings in the Italian Maritime Alps. Bordighera, Gibelli.

BICKNELL. C. (1903). Further exploration in the region of the prehistoric rock engravings in the Italian Maritime Alps. Bordighera, Gibelli.

BICKNELL C. (1909). Nuovo contributo alla cognizione delle incisioni osservate nelle alte valli delle Alpi Marittime. Genova, Ciminiago.

BICKNELL C. (1913). A guide to the prehistoric rocks engravings in the Italian Maritime Alps. Bordighera, Bessona.

BLANC E. (1879). Étude sur les sculptures préhistoriques de Val d'Enfer. Mémoires de la Société des Sciences naturelles et historiques des Lettres et des Beaux-arts de Cannes et de l'arrondissement de Grasse, t. VII, 1877-1878, Cannes, p. 72-89.

BLEICHMAR D. (2009). Visible Empire: scientific expeditions and visual culture in the Hispanic enlightenment, Postcolonial studies, vol. 12, n 4, p. 441-466.

BORDIER A. (1878). Les sciences anthropologiques à l'exposition universelle. in La Nature, Sixième année, deuxième semestre, 1878, n 261-287, p. 129-131, 210-214, 358-362, 408-410.

Clugnet L. (1877). Sculptures préhistoriques situées sur les bords des Lacs des Merveilles. Matériaux pour l'histoire primitive et naturelle de l'Homme, $2^{\mathrm{e}}$ série, t. VIII, p. 379387.

CLUGNET L. (1879). Incident à propos des sculptures sur rochers du Lac des Merveilles. Matériaux pour l'histoire primitive et naturelle de l'Homme, $2^{\mathrm{e}}$ série, t. X, p. 235-239.

DAWES B. (3003). La rivoluzione turistica. Thomas Cook e il turismo inglese in Italia nel XIX secolo, Napoli-Roma, Edizioni Scientifiche Italiane.

FODÉRÉ F.-E. (1821). Voyage aux Alpes Maritimes, ou Histoire naturelle, agraire, civile et médicale du comté de Nice et pays limitrophes. Paris, F. G. Levrault.

Gioffredo P. (1839). Storia delle Alpi Marittime, Torino, Stamperia Reale. 
GRÄSLUND B. (1987). The Birth of Prehistoric Chronology. Dating methods and dating systems in nineteenth-century Scandinavian archaeology. Cambridge, Cambridge University Press, p. 131.

ISSEL A. (1901). Le rupi scolpite nelle alte valli della Alpi Marittime, Bullettino di Paleontologia italiana, IIIè serie, t. VII, anno XXVII, n 10-12, p. 218-259.

ISSEL A. (1908). Liguria preistorica, Atti della Società Ligure di Storia Patria, Genova.

Latour B. (2007). Le "pédofil" de Boa-Vista - Montage photo-philosophique, Petites leçons de sociologie des sciences, Paris, La Découverte.

LuMLeY H. (de) et al. (2011). La montagne sacrée du Bégo, CNRS Éditions, Paris.

Moggridge J. T. (1866). Menton et ses environs par un touriste anglais, accompagné d'un panorama des montagnes, de M. Moggridge. Paris, Librairie Nouvelle.

MogGridge J. T. (1866). Monstrosities in Ophrys Insectifera, Linné. The journal of Botany, British and foreign, vol. IV, p. 167-168.

MogGRIDGE J. T. (1867). Some abnormal forms of Ophrys. The journal of Botany, British and foreign, vol. V, p. 316-318.

Moggridge J. T. (1871). Contribution to the Flora of Mentone and to a winter Flora of the Riviera including the coast from Marseille to Genova, London, Reeve and $\mathrm{C}^{\circ}$.

MogGridge M. (1869). The Meraviglie. International Congress of Prehistoric Archaeology, London, Longmans, Green and $\mathrm{C}^{\circ}$, p. 359-363.

MogGridge M. (1867). On the zones of Coniferae, from the Mediterranean to the crest of the Maritime Alps, The Journal of Botany, British and foreign, vol. V, n¹, p. 48-50.

MogGridge M. (1875). Les Merveilles, Revue Archéologique, nouvelle série, XXIX, pp. 370373. Planches XV-XVI

RIVIÈRE É. (1879). Gravures sur roche des Lacs des Merveilles au val d'Enfer, Association française pour l'Avancement des sciences. Congrès de Paris de 1878, Comptes rendus de la $7^{e}$ session, Paris, p. 783-793.

SCHLANGER N. (2010). Series in progress: antiquities of nature, numismatics and stone implements in the emergence of prehistoric archaeology, History of Science, XLVIII, p. 344369.

WeSTROPP HODDER M. (1869). On rock carvings, International Congress of Prehistoric Archaeology 1868, London, Longmans, Green and C ${ }^{\circ}$, p. 47-55. 


\section{Légende des figures}

Figure 1 : Moggridge, production naturaliste de figures singularisées. PLATE V. 1869.

Matthew Moggridge, "The Meraviglie», International Congress of Prehistoric Archaeology, London, Longmans, Green and co, 1869. pp. 359-363.

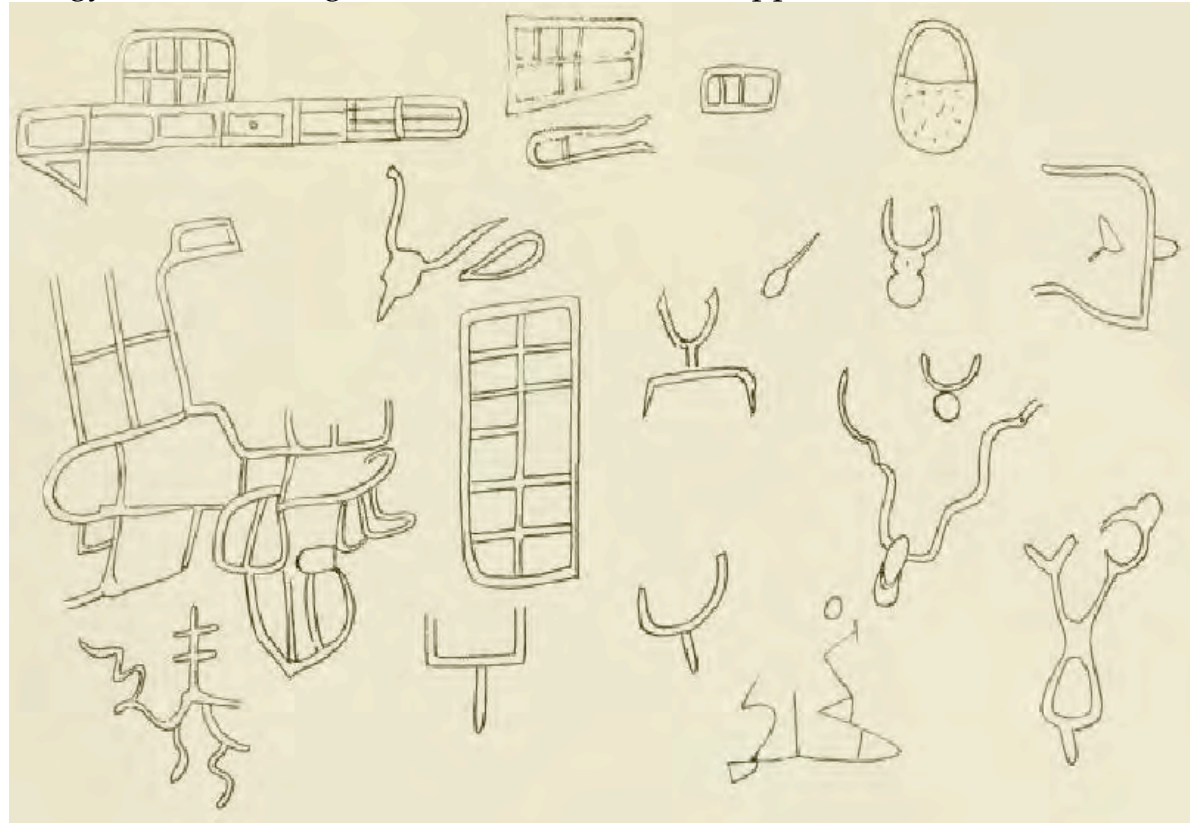

Figure 2 : Bovinés et caprinés, les types finement différenciés de Clugnet. 1877.

Les sculptures sur rochers des lacs des Merveilles, Planche III. 1877. Léon Clugnet, "Sculptures préhistoriques situées sur les bords des Lacs des Merveilles. », Matériaux pour l'histoire primitive et naturelle de l'Homme, $2^{\text {éme }}$ série, Tome VIII, août 1877, pp. 379-387.

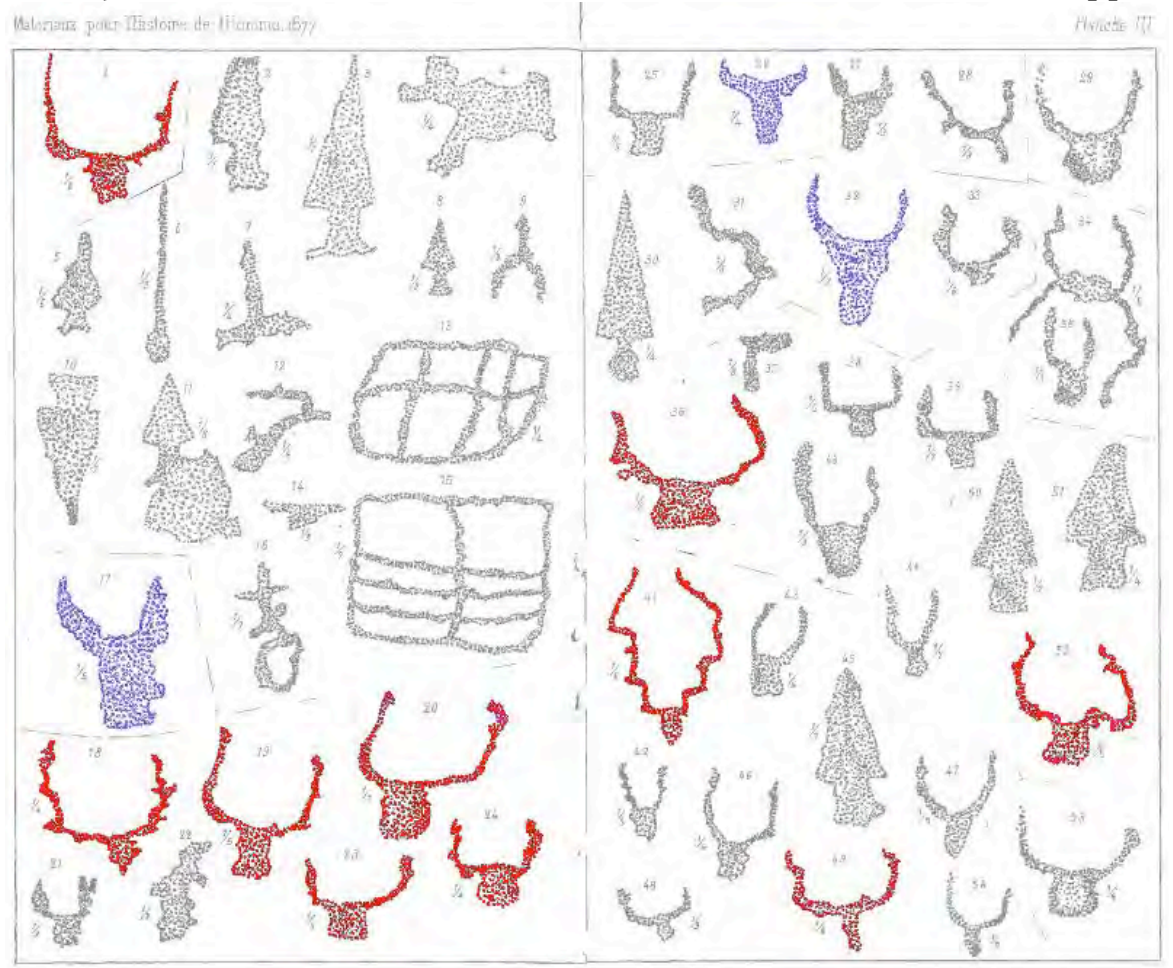

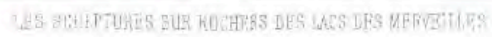


Figure 3: Différents types dans le groupe des armes instruments et outils de Rivière. 1879 Gravures sur roches des Lacs des Merveilles en Italie. T. VII. Pl. XI. 1879. Émile Rivière, "Gravures sur roche des Lacs des Merveilles au val d'Enfer », Association française pour l'Avancement des sciences. Congrès de Paris de 1878, Comptes rendus de la 7 session. Paris, 1879. pp. 783-793.

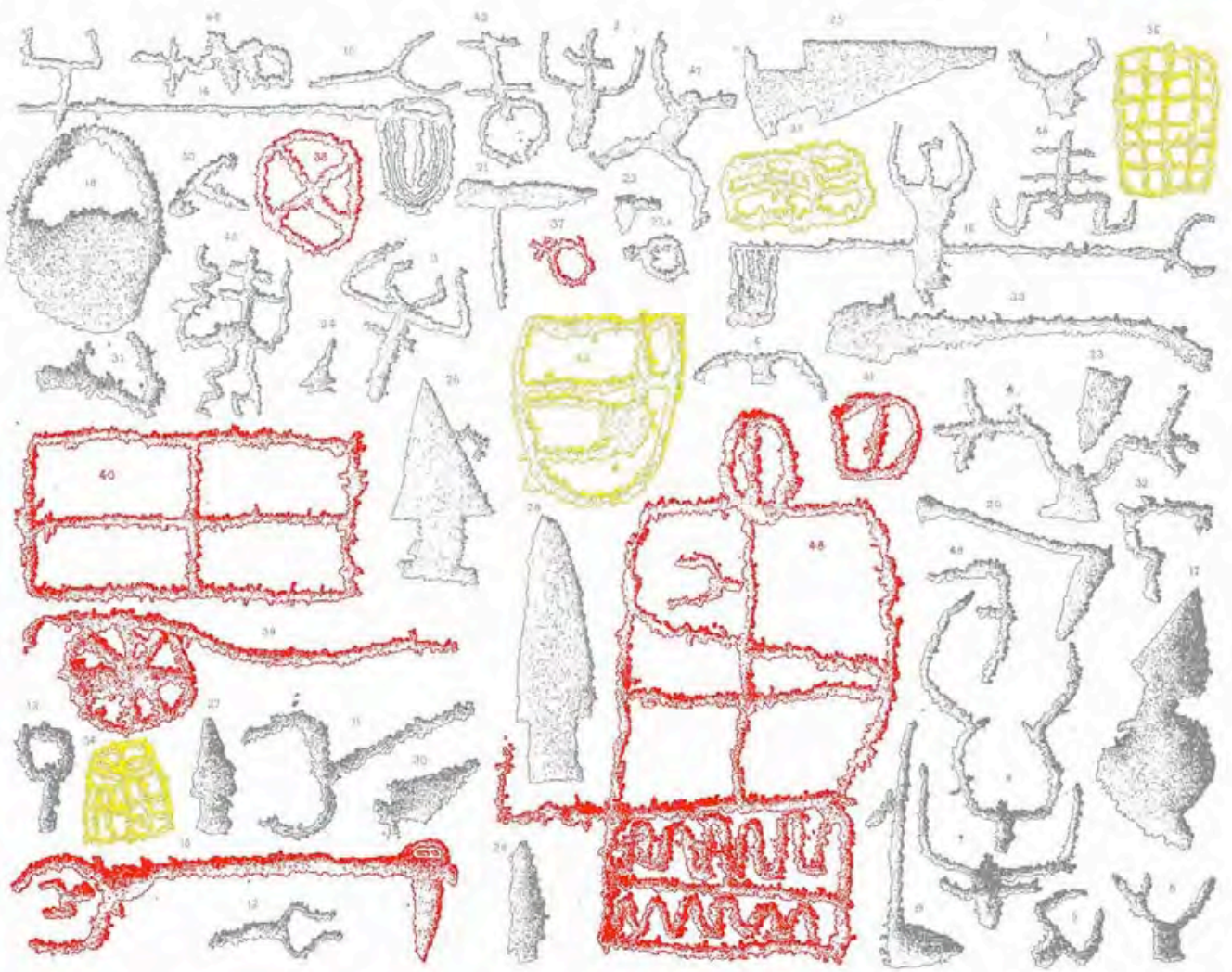


Figure 4 : Amulettes de Blanc, 1877-1878

Sculptures préhistoriques du Val d'Enfer. 1877, Edmond Blanc, «Étude sur les sculptures préhistoriques de Val d'Enfer », Mémoires de la Société des Sciences Naturelles et Historiques des Lettres et des Beaux-arts de Cannes et de l'arrondissement de Grasse. Tome VII, 1877-1878, Cannes, 1879. pp. 72-89.

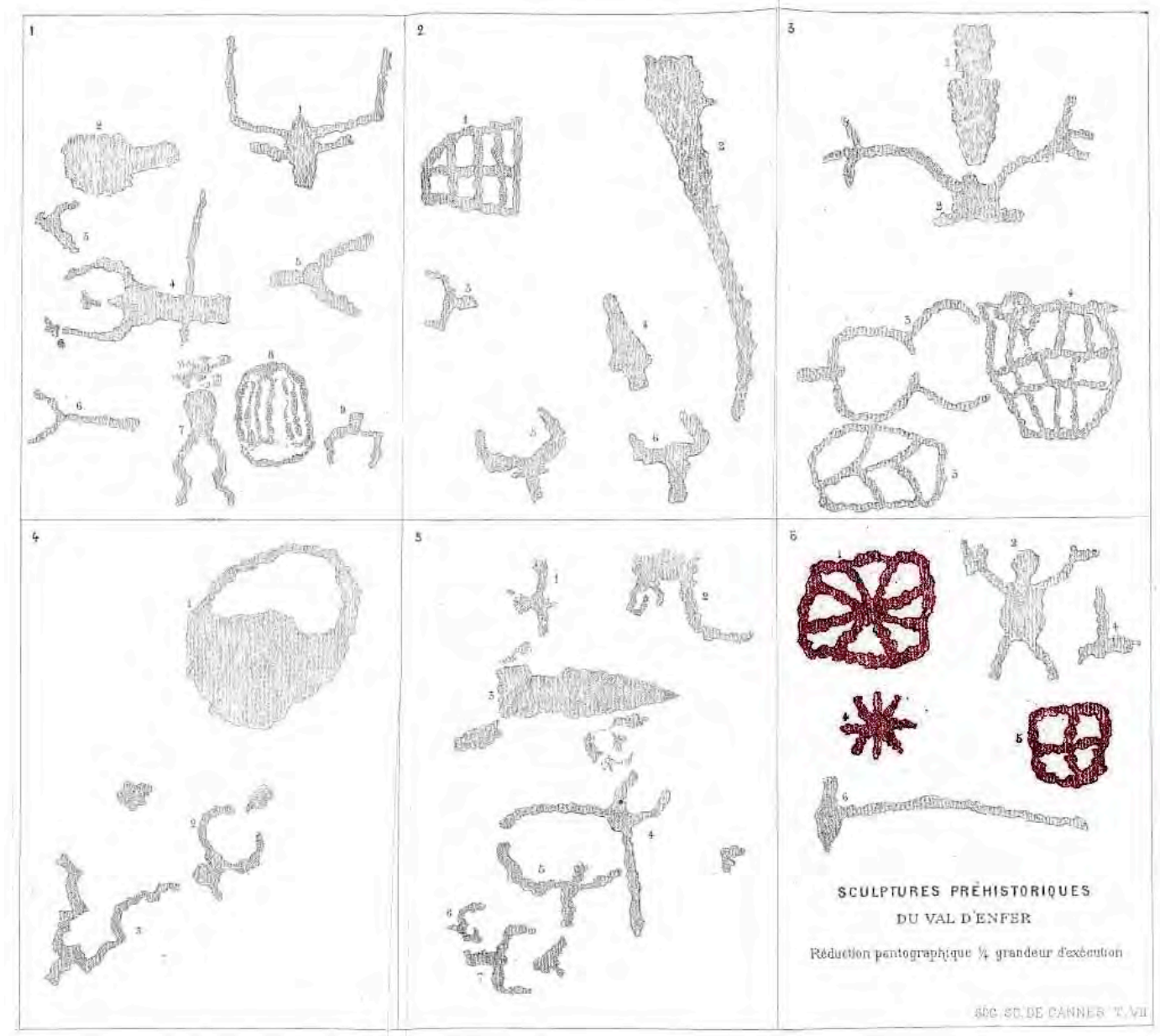


Figure 5 : Les taxons des animaux avec des cornes créées par Bicknell. 1913.

Various types of horned beasts. 1913, Clarence Bicknell, A guide to the prehistoric rocks engravings in the Italian Maritime Alps. Bordighera, Bessona, 1913.

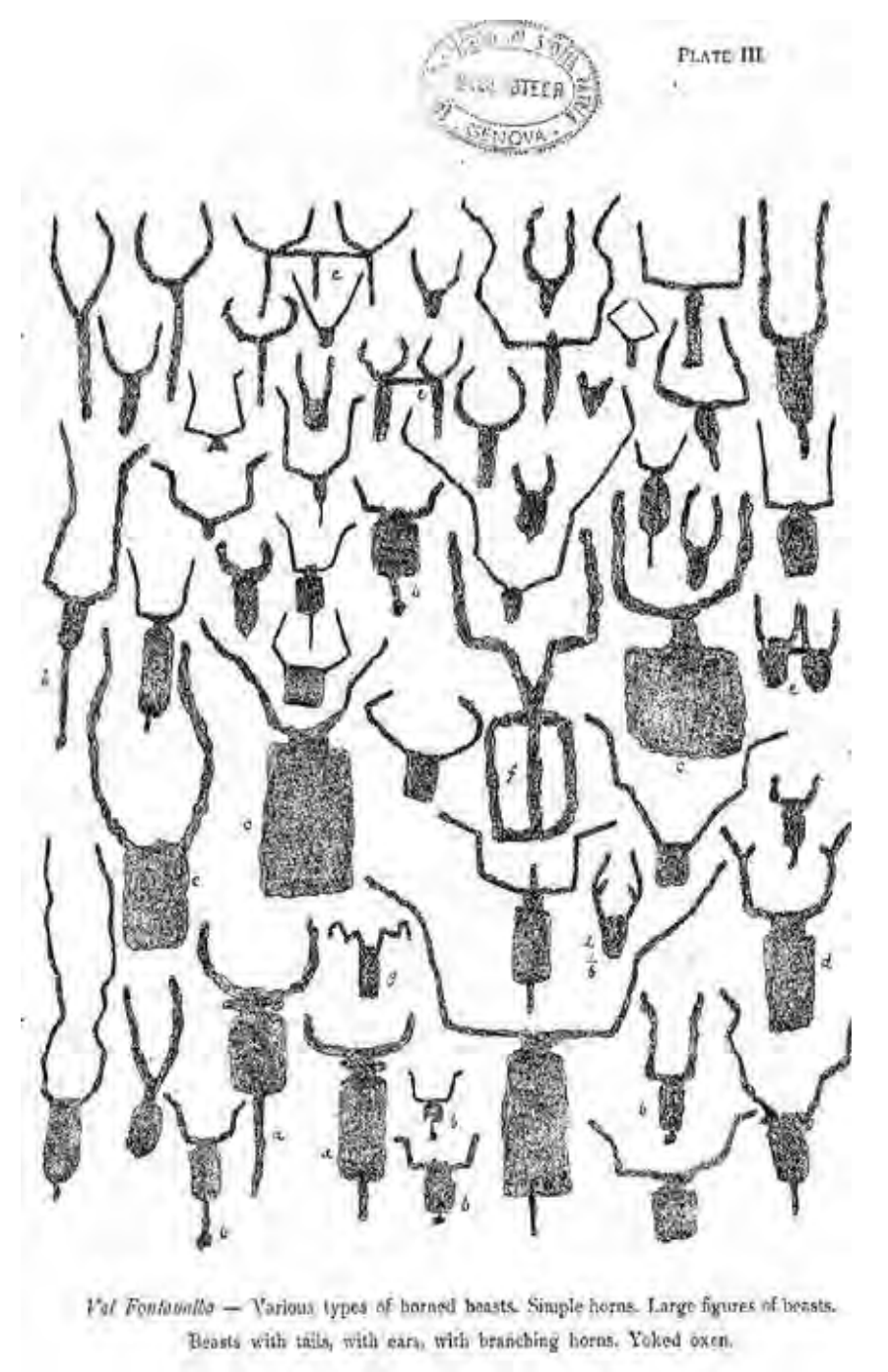




\title{
Communication visuelle autour du Camp du Château à Salins-les-Bains (Jura, France) : mise en évidence d'un réseau de relations visuelles à l'âge du Bronze
}

\author{
Estelle GAUTHIER \\ Maître de Conférences, Université de Franche-Comté, \\ CNRS UMR 6249, Chrono-Environnement, Besançon \\ Jean-François PININGRE \\ Conservateur du Patrimoine, DRAC Franche-Comté, \\ CNRS UMR 6298 ARTEHIS, Dijon \\ avec la collaboration de Hervé GRUT
}

Extrait de : Olivier BUCHSENSCHUTZ, Christian JEUNESSE, Claude MORDANT et Denis VIALOU (dir.),
Signes et communication dans les civilisations de la parole, Paris,
Édition électronique du CTHS (Actes des congrès des sociétés historiques et scientifiques), 2016.

Cet article a été validé par le comité de lecture des Éditions du CTHS dans le cadre de la publication des actes du $139^{\text {e }}$ Congrès national des sociétés historiques et scientifiques tenu à Nîmes en 2014.

\begin{abstract}
Résumé
Des campagnes de prospections systématiques menées depuis plusieurs années dans le secteur du Camp du Château à Salins-les-Bains ont permis la mise au jour de 40 dépôts de l'âge du Bronze dans des contextes topographiques particuliers. La bonne précision de leur localisation permet de mesurer les liens de perceptibilité entre les lieux de dépôt et des points marquants du paysage naturel et social de l'âge du Bronze, en particulier des sites de hauteur. La topographie de la région a en effet favorisé la constitution d'une véritable toile de relations visuelles tout autour du Camp du Château. Les dépôts semblent marquer les frontières de l'espace visible depuis celui-ci. La mise en place d'une forme de communication visuelle entre les sites a pu permettre de soutenir la mémoire communautaire afin d'entretenir les traditions. Ainsi les choix d'implantation des dépôts avaient-ils pour objectif de baliser l'espace social et culturel associé au camp de hauteur fortifié ?
\end{abstract}

Mots-clés : dépôts de bronzes, âge du Bronze, visibilité, camp de hauteur, actes sociaux, mémoire communautaire, paysage sacré

\begin{abstract}
Ongoing prospection of the area around the Camp du Château at Salins-les-Bains has brought to light 40 Bronze Age hoards in notable topographical contexts. Their precise location has allowed us to define the perceptible link between the hoards' location and particular landmarks in the natural and social landscape of the Bronze Age and in particular high altitude sites. The topography of the area seems to have conducive in establishing a web of visual relations around the Camp du Château. The hoards mark borders visible from the Camp du Château and contribute to a kind of visual communication needed to support the collective memory that maintains traditions. Do the choices in hoard location demarcate the social and cultural space associated with this fortified site? (traduction Rebecca Peake)
\end{abstract}

L'approche de la dynamique spatiale des dépôts de bronzes repose sur une documentation tributaire $\mathrm{du}$ hasard de découvertes souvent anciennes, d'ensembles inégalement documentés, voire incomplets. La région de Salins-les-Bains (Jura) apparaissait jusqu'alors peu documentée par rapport à d'autres secteurs présentant des conditions équivalentes dans la densité des sites, le contexte géographique, la présence de sources de matières premières comme le sel dans la région de Lons-le-Saunier par exemple. Seuls y étaient connus quelques petits dépôts du Bronze ancien et de la fin du Bronze final (Millotte 1963, Piningre 1998). 
Un programme de prospections systématiques au détecteur, conduit dans le cadre légal et formel d'une étude de l'environnement archéologique du site fortifié de hauteur du Camp du Château, est venu sensiblement modifier cet état. En bordure du Jura central, cette zone, aux reliefs contrastés (fig. 1), contrôlée par cinq sites de hauteur fortifiés occupés à différentes périodes de l'âge du Bronze, constitue un point de passage privilégié entre les plaines de la Saône et la vallée de la Loue, d'une part, les plateaux du Jura central et le Plateau suisse d'autre part (Piningre et Grut 2009). Les sources salées des marnes du Trias, exploitées dès le Néolithique (Pétrequin et al. 2001), représentent un atout supplémentaire propice au développement de l'économie et des échanges.

Le recensement et la fouille de 40 dépôts datant principalement de la fin du Bronze moyen (Bz C2) et du début du Bronze final (Bz D/Ha A1) a autorisé dans un premier temps un réexamen de la question de l'insertion des dépôts dans le cadre d'une microrégion. À partir d'une documentation représentative, ce programme a permis de recueillir de précieuses informations concernant la localisation, le contexte de déposition et la composition de ces ensembles par la prise en compte d'effectifs complets et non sélectionnés. Cette concentration de dépôts constitue un phénomène rare qui n'est sans doute pas étranger à cette situation géographique (fig. 2).

\section{Composition et chronologie des dépôts}

Les dépôts de Salins sont composés d'un nombre d'objets compris entre quelques unités et près de 300 individus, soit une moyenne de 35 objets par dépôt, pour un poids moyen de $2 \mathrm{~kg}$ qui peut atteindre pour les plus importants entre 6 et $9 \mathrm{~kg}$ (fig. 3). La plupart d'entre eux associent à des fragments de lingots, un lot d'objets manufacturés, fragmentés, parmi lesquels prédominent les outils (haches, faucilles) et, dans de plus faibles proportions, les parures (épingles, bracelets) et quelques armes (poignards, lames d'épées). Ils peuvent entrer ainsi dans la définition des "dépôts de recyclage ». Douze dépôts, ne comprenant que des fragments de lingots, seuls ou associés à des fragments d'objets non datables, offrent des caractères analogues aux précédents, mais ne peuvent être situés avec toute la précision chronologique souhaitable. Enfin, quelques dépôts évoquant des assortiments de parures féminines entières, appariées, et un petit dépôt de déchets d'or se distinguent plus nettement des précédents et peuvent refléter un rôle social différent.

Dans le détail, l'analyse de ces dépôts ne répond sans doute pas à une seule logique. La confrontation du poids moyen et des effectifs permet de distinguer des ensembles pondéreux de plusieurs kilos qui diffèrent des dépôts peu pondéreux, où la fragmentation est maximale. La masse de quelques centaines de grammes de certains d'entre eux, ou les quelques ensembles de parures et d'or, correspondent difficilement à l'image du dépôt de fondeur classique.

Indépendamment des distinctions que nous venons de souligner, on remarquera que ces dépôts appartiennent tous à une tranche chronologique précise, comprise entre le Bronze moyen ( $\mathrm{Bz}$ B / C) et la période initiale du Bronze final (Bz D2/Ha A1), et que leur nombre fluctuant connaît un accroissement qui place les $2 / 3$ des ensembles datables entre le Bz C2 et D1 (fig. 4). Durant toute la période, la modification dans le temps de la composition des dépôts pourrait être comprise dans le sens d'une évolution de pratiques codifiées. Un accroissement de la fragmentation et une évolution des composants sont ainsi nettement perceptibles (Piningre et Grut 2009, Piningre et al., à paraître). Les dépôts les plus anciens sont composés de petits lots d'objets complets ou peu fragmentés et peu diversifiés (généralement des haches). Ils font place aux dépôts pondéreux de la fin du Bronze moyen et du début du Bronze final (Bz C2-D1), où les fragments de lingots sont majoritaires, alors que dans ceux, moins nombreux, du début du Bronze final, les objets très fragmentés dominent et la proportion des lingots diminue sensiblement (Piningre et Grut 2009). 


\section{Localisation et contexte de déposition}

La zone considérée (fig. 2) est centrée sur une aire de $10 \mathrm{~km}$ de diamètre autour de Salinsles-Bains, comprise entre les communes de Malans au Nord-Est, Aresches au Sud et Marnoz à l'Ouest. Au centre, le Mont Poupet domine le secteur à $808 \mathrm{~m}$ d'altitude. Il ne semble pas avoir été occupé, mais quelques dépôts anciens y ont été signalés (Millotte 1963, p. 341). Le Camp du Château, occupé à partir de la fin du Bronze moyen et au début du Bronze final (Bz C2/D1), a été abondamment étudié et semble dès cette époque occuper un rôle majeur (Piningre et Ganard 2004; Piningre et Grut 2009). Il est contemporain de la plupart des 40 dépôts trouvés lors des campagnes de prospection (fig. 1), qui sont datés essentiellement de la même période. Le sud de la zone est parcouru par la Furieuse, un affluent de la Loue, qui passe entre le Mont Poupet et le Camp du Château. Outre les 5 sites de hauteur fortifiés proches du Mont Poupet (à moins de $5 \mathrm{~km}$ de la ville actuelle de Salins), la zone compte encore trois autres sites de hauteur à une dizaine de kilomètres. La datation des occupations de ceux-ci reste floue. Même si certains d'entre eux semblent abandonnés à la fin du Bronze ancien, peut-être ont-ils continué à occuper une place dans le paysage culturel?

La localisation des dépôts dans la zone d'étude (fig. 2) permet de distinguer plusieurs groupes:

- immédiatement au sud du Mont Poupet, sur la commune de Saint-Thiébaud, quatre dépôts sont très proches les uns des autres (Le Poirier au Loup, dépôts 1, 2 et 3 et Mouleyrand).

- à l'est du Mont Poupet, de part et d'autre du cours du Todeur, six dépôts se trouvent en limite des communes de Salins-les-Bains et de Saizenay, aux lieux-dits, Bois-Bovard, Bois de Séry et Côte Chenien.

- au sud du Camp du Château, c'est un ensemble de quatorze dépôts qui se trouvent alignés le long d'une crête sur la commune de Pretin, face aux camps de Bracon et de Château.

- au nord du Camp du Château, deux dépôts ont été découverts sur la commune de Marnoz, et deux autres non loin, au lieu-dit Granges-Feuillet.

- au sud de Salins-les-Bains, quatre autres trouvailles proches sont localisées aux lieuxdits Côte de Thésy et Côte-en-Velet. Un dépôt a été découvert beaucoup plus loin au sud, à Aresches.

- enfin, tout au nord de la zone prospectée, un ensemble de huit dépôts est dispersé sur les communes de Chay, Bartherans, Cussey-sur-Lison, Malans (3 dépôts) et Eternoz (2 dépôts).

Les contextes topographiques des lieux de dépôt présentent des caractères particuliers propres à la catégorie des Abhangdepots selon la classification de T. Soroceanu (1995, p. 23 ; 2012), correspondant à deux types de localisations. À Pretin, Salins, SaintThiébaud, Saizenay, la plupart d'entre eux se retrouvent ainsi à proximité d'escarpements rocheux, en bordure du plateau, dans des fissures ou en contrebas sur les talus d'éboulis de corniches calcaires. Quelques-uns sont situés sur des crêtes calcaires de lapiaz démantelées par l'érosion, entre des blocs volumineux à Aresches, Salins-Bois Bovard. Ainsi, ces localisations s'identifient souvent avec des repères topographiques naturels, bien visibles en milieu déboisé (corniches calcaires, promontoire rocheux, pointes de lapiaz en relief).

Le faible enfouissement des objets (entre 5 et $25 \mathrm{~cm}$ de profondeur en moyenne, voire en surface pour certains), sous la forme de concentrations lâches sur des superficies de quelques $\mathrm{m}^{2}$, exclut l'existence de dépôts en urne, de dépôts groupés en fosse ou en contenant périssable profondément enfouis. Les objets formant un même ensemble peuvent être éparpillés, ou quelquefois groupés en plusieurs lots de quelques individus. L'existence d'objets déposés dans les fissures d'une corniche rocheuse est attestée à 
Saizenay, alors que d'autres objets retrouvés en contrebas dans une diaclase argileuse et entre des blocs du talus d'éboulis semblent avoir été naturellement dispersés par la suite. Une mise en place analogue est vraisemblable à Pretin 12. La position d'une majorité des lots d'objets du secteur salinois sur des surfaces planes présentant un substrat rocheux à faible couverture argileuse semble exclure toute action d'érosion importante, naturelle ou anthropique - liée à l'agriculture par exemple - justifiant cette dispersion. Ces caractères sont donc difficilement compatibles avec l'occultation d'un lot d'objets profondément enfoui suivant le modèle classique des cachettes de métal. Ils accréditeraient la volonté d'un faible enfouissement initial, voire la dispersion superficielle des objets dès leur abandon ou encore une déposition hors sol (dans un tronc d'arbre ou associée à une construction périssable par exemple). Cette disposition rendrait alors possible des actes de dépôts effectués en une seule fois ou successivement, échelonnés dans le temps. Les mêmes observations ont été faites pour d'autres découvertes récentes, comme le dépôt 6 de Rabenwand, (Styrie, Autriche, Windholz-Konrad 2005). Les dépôts de Rabenwand sont très similaires à ceux étudiés ici tant par leur position topographique, que la proximité des dépôts, la composition des assemblages, la dispersion des objets et leur faible profondeur, à la différence près qu'ils ont été couverts de blocs de pierres.

En première analyse, la répartition spatiale des dépôts retient également l'attention. Les trois groupes principaux de Pretin, Salins-Côte de Thésy et Saint-Thiébaud rassemblent respectivement sur quelques centaines de mètres l'essentiel des dépôts identifiés dans le secteur de Salins (fig. 2). Dans chacun de ces ensembles, les dépôts espacés de quelques dizaines ou de quelques centaines de mètres délimitent généralement des zones étendues plutôt que des emplacements ponctuels. Parmi ceux-ci, le secteur de Pretin avec quatorze concentrations d'objets, occupe une place privilégiée où la proximité du Camp du Château, visible à $1 \mathrm{~km}$ au Nord, ne semble pas fortuite. Il se distingue par un nombre inégalé de dépôts, ainsi que par le regroupement exceptionnel de six concentrations d'objets placées au voisinage immédiat d'une aiguille rocheuse, détachée de la falaise et formant un point de repère évident dans le paysage.

À l'échelle de la zone d'étude, on remarque aussi que les dépôts forment deux groupes centrés sur deux axes de pénétration du massif jurassien par deux affluents de la Loue, les vallées de la Furieuse au sud et du Lison au nord. Chacune de ces concentrations correspond aussi avec des zones marquantes du paysage soulignant le contact entre la vallée et le plateau.

\section{Les dépôts de bronzes : entre mémoire et perceptibilité}

Si l'image que nous percevons des traditions au sein des sociétés protohistoriques repose sur des vestiges dont l'interprétation met en avant le caractère répétitif et symbolique, principalement dans des lieux à vocation funéraire ou cultuelle (voir une synthèse de ces indices dans Van Dyke et Alcock 2003), certains de ces actes communautaires ont laissé des traces plus ou moins bien interprétables (Bradley 2000). Tel est notamment le cas des dépôts de l'âge du Bronze, placés dans des contextes isolés, à l'écart des habitats et des nécropoles. Tandis que certains y voient des quantités de métal retirées temporairement des circuits de consommation pour répondre à des objectifs économiques, d'autres les interprètent comme le résultat d'actes sociaux voire cultuels (sur l'interprétation des dépôts, voir par ex. Bradley 1990 ; Hansen 1994 ; Kristiansen 2000 ; Fontijn 2002). Sans préjuger que tous les dépôts correspondent à ce type d'activité, on peut raisonnablement penser que certains d'entre eux ont effectivement pu répondre à des préoccupations touchant au domaine du symbolique et de l'expression sociale, de par le nombre, la nature et l'état des objets conservés, mais aussi leur contexte d'enfouissement (voir également une très récente étude de dépôts "de fondeur ", démontrant qu'ils peuvent être considérés comme des accumulations d'objets votifs à long terme, intégrés à des pratiques sociales, Dietrich 2014). 
Or, les caractéristiques décrites ci-dessus de la quarantaine d'ensembles étudiés ici, que ce soit l'évolution de leur composition et de l'état des pièces, leur contexte géographique et leur emplacement topographique, mettent en avant l'idée d'une pratique ritualisée. En déposant des lots d'objets fragmentés inutilisables en des endroits remarquables, facilement accessibles et repérables, il ne s'agirait donc pas seulement de soustraire définitivement une masse de métal à la consommation, mais peut-être aussi de la placer sous une protection spécifique en un lieu consacré. Les transformations de ces pratiques dans le temps ont pu, quant à elles, résulter aussi d'une fluctuation des dynamiques économiques et des approvisionnements en métal.

Si l'on admet donc que ces dépôts aient pu avoir un caractère définitif et un rôle social, on peut considérer qu'ils sont le résultat de pratiques communautaires répétitives, dont on peut supposer qu'elles reposent sur un ensemble de traditions partagées par les membres d'une même communauté. En effet, les coutumes reproduisent des gestes devant correspondre à des idées ou des mythes se transmettant de génération en génération, en tant qu'héritage culturel et vecteur de l'identité communautaire. Les actes qui en résultent se fondent donc sur un ensemble d'interactions et d'interprétations sociales d'où émerge un sens commun donné aux objets et aux lieux (Mead 1938). Or, ces traditions ne bénéficiant pas de supports écrits, elles devaient reposer sur une transmission orale afin de subsister dans la mémoire communautaire (sur le rôle de la mémoire sociale, voir Van Dyke et Alcock 2003).

Les dépôts étant destinés à être enfouis et les lieux n'ayant sans doute pas été aménagés durablement en surface, seule une communication orale a pu permettre de transmettre leur souvenir (Fontijn 2007). Or, les dépôts peuvent en eux-mêmes être vus comme des actes de célébration de la mémoire (Bradley 1990, Van Dyke et Alcock 2003). De même, les objets qu'ils renferment, de par leur propre histoire, leur "biographie » (Kopytoff 1986 ; Gosden et Marshall 1999 ; Fontijn 2002), offrent un accès au passé (Rowlands 1993). L'exemple du dépôt de Salisbury est particulièrement significatif (Stead 1998). Ce lot d'objets métalliques, enfoui au Second âge du Fer est issu de la récupération de plusieurs dépôts de l'âge du Bronze, associés à des objets spécifiquement votifs, comme des boucliers et des chaudrons miniatures et finalement déposés au fond d'une même fosse. On ne sait pas durant quel laps de temps les dépôts anciens ont été collectés, ni sur quelle aire géographique. Ce cas très particulier illustre non seulement le lien entre la déposition d'objets métalliques et le domaine religieux, mais également la place de la mémoire dans ces pratiques : leur persistance et l'impact de l'ancienneté de ces objets sur la valeur qui leur était attribuée. La notion de mémoire apparaît donc comme essentielle lorsque l'on parle de dépôts (Fontijn 2002). Nous sommes ici à la limite entre les pratiques de « mémoire inscrite » (actes communautaires visibles) et de «mémoire incorporée » (actes secrets et éphémères) de M. Rowlands (1993).

Néanmoins, la mémoire collective a une durée limitée (Bradley 2003). Un soutien visuel pourrait avoir permis de l'entretenir. En effet, la vue, premier vecteur de communication après la parole, s'intègre dans les processus d'échanges sociaux et permet d'établir des liens entre les individus, mais aussi avec des objets ou des lieux. Elle permet de conserver, rappeler et partager le souvenir des actes passés. Concernant l'histoire d'une population sans écriture :

«Un élément visuel est presque toujours nécessaire pour maintenir vivante la mémoire soutenue uniquement par des traditions orales. » (Person 1962, p. 468)

Ainsi des objets mobiliers ou des monuments ont vraisemblablement été utilisés pour conserver la mémoire d'actes passés (Bradley 2003).

Concernant les dépôts, ce n'était bien entendu pas les objets eux-mêmes qu'il s'agissait de voir, mais plutôt les endroits qui ont été le cadre des rites perpétués par les membres de la communauté, parmi lesquels le sacrifice (l'enfouissement définitif) d'objets de bronze 
pourrait n'être que la dernière étape et la seule trace archéologique qui nous soit parvenue.

Ici, le fait de voir les lieux précisément n'est pas tant important que le fait de pouvoir les apercevoir ou se les représenter mentalement. Leur "présence » dans le paysage permet de les intégrer au quotidien et de maintenir vivace le souvenir de ces lieux qui avaient alors peut-être acquis un caractère sacré. On peut en effet penser que l'endroit conservait une importance pour la société, au moins pour quelque temps. Ils sont chargés émotionnellement et montrent un engagement social dans le paysage (Schama 1996, Van Dyke et Alcock 2003, Harmanşah 2014). D'une part, la sélection de contextes topographiques particuliers montre que le choix de leur emplacement n'était pas anodin et d'autre part, le positionnement des dépôts suivants a pu être conditionné par celui des précédents. On le voit notamment par l'accumulation de dépôts sur certains sites : Pretin, 14 dépôts; Rabenwand, au moins 7, (Windholz-Konrad 2005) ; Bullenheimer Berg, au moins 19, (Diehm, Falkenstein 2012). Par ailleurs, si les enfouissements n'ont pas été faits en une seule fois (voir ci-dessus), il était nécessaire de se souvenir de leur emplacement.

De récentes études des dépôts (voir notamment Fontijn 2002 ; Hansen et al. 2012) ont révélé que leur emplacement s'intègre dans le paysage naturel et anthropisé. Bien que les dépôts ne soient pas marqués au sol, il est probable qu'un moyen de se souvenir et de repérer leur emplacement fut, comme ici, leur proximité avec des éléments naturels particuliers. Et à l'instar des dépôts de la région de Salins-les-Bains, les choix topographiques (Soroceanu 2012) mais aussi leur position par rapport à d'importants axes de circulation ou l'exploitation de ressources, notamment en sel, sont des éléments souvent constatés (par exemple Windholz-Konrad 2002, 2005, 2010 ; Dietrich 2013). On connaît aussi plusieurs cas d'associations de dépôts avec des sites de hauteur fortifiés ; l'exemple de Bullenheimer Berg est l'un des plus marquants (Diemer 1995).

Dans ce contexte, l'importance du lien visuel avec les différents éléments constituant le paysage social devient claire : les sites cultuels peuvent en effet baliser le paysage. Au moment des cérémonies, le contact visuel avec les autres sites pouvait permettre une prise en compte plus globale de l'espace cultuel. En effet, comme le souligne D. Fontijn (2002), il est préférable de ne pas considérer les lieux de déposition comme indépendants les uns des autres, mais il est plus approprié de parler en termes de "zones de déposition ». L'idée d'un balisage du paysage a déjà été proposée dans un contexte topographique similaire, pour des sanctuaires de pics en Crête à l'âge du Bronze (Peatfield 1983, 1994). L'intervisibilité aurait tenu un rôle important dans la gestion du paysage religieux et politique (Soetens et al. 2002). La perception des anciens lieux de dépôt servirait en effet à la commémoration des activités passées et ces lieux seraient devenus des jalons visuels pour les participants à ces activités. Par ailleurs, selon A. Peatfield (1994, p. 25), à un niveau régional, l'intervisibilité des sanctuaires de pics offre une opportunité pour l'expression de l'unité rituelle qui a pu dépasser les frontières politiques. Cet exemple apporte deux pistes intéressantes: d'une part, la visibilité peut jouer un rôle notable dans l'implantation des sites à caractère religieux et d'autre part, ces sites forment un tout dans le paysage cultuel d'un territoire ou d'une région.

Or, la topographie de la région des Salins, très contrastée, offre de beaux panoramas dans lesquels il est aisé de choisir des lieux bien visibles ou, au contraire, cachés ou encore des lieux à partir desquels on peut voir tel ou tel autre point important dans le paysage. Il apparaît donc intéressant de chercher si des liens de visibilité ou de perception peuvent être identifiés entre les lieux de déposition et différents éléments marquants du paysage social : l'habitat de hauteur fortifié situé sur le site du Camp du Château est en position centrale et concentre les activités de l'époque ; le Mont Poupet domine visuellement la zone à $808 \mathrm{~m}$ d'altitude; plusieurs autres camps de hauteur plus petits et pour certains occupés plus anciennement ont pu conserver un rôle dans la mémoire collective à cette époque. 


\section{Une implantation des dépôts fondée sur un système de relations visuelles?}

Pour répondre à cette question, une approche exploratoire a été menée afin de tester la visibilité depuis et en direction de chacun des lieux concernés, à l'aide d'un Système d'Information Géographique. La méthode a été développée dans les années 1990 (voir notamment Fisher 1996; Wheatley et Gillings 2000 ; Lake et Woodman 2003). Le protocole d'étude mis en place consiste à calculer les zones visibles depuis chacun des sites et à comptabiliser les relations visuelles. Le MNT utilisé est celui de la BD ALTI de l'IGN, avec une résolution de $25 \mathrm{~m}$. La notion de perception étant plus imprécise que la visibilité, une marge supplémentaire de 100 mètres autour des zones de visibilité a été prise en compte.

Les analyses mettent en évidence une forte intervisibilité des lieux de dépôt puisque la plupart sont visibles depuis 8 autres sites en moyenne, et inversement (fig. 5). Certains ont une bien meilleure visibilité, notamment ceux de Pretin 14 et Thésy 3, d'où il est possible d'apercevoir jusqu'à 21 lieux de dépôt. Les groupes de dépôts de Pretin et de Saint-Thiébaud sont les mieux perceptibles depuis les autres emplacements car on peut les apercevoir depuis une douzaine de sites.

Mais il est clair que ces relations ne concernent quasiment que le centre de la zone étudiée, sur l'actuelle Salins-les-Bains et les communes limitrophes. Il est intéressant de noter qu'il existe aussi une forte inter-visibilité entre les différents groupes de dépôts situés de part et d'autre du Camp du Château et du Mont Poupet (Saint-Thiébaud, Clucy, Thésy et Pretin). Ces relations donnent l'image d'un système de liens visuels complémentaires, visant à couvrir tout le secteur immédiat du Camp du Château (fig. 6).

Concernant la position des lieux de dépôt par rapport aux points marquants du paysage social de l'époque, on observe également de très bonnes relations visuelles, en particulier avec le Camp du Château et le Mont Poupet, privilégiés bien entendus par leur position dominante leur conférant une large emprise visuelle. Mais il faut noter que pratiquement tous les dépôts du centre de la zone étudiée peuvent être aperçus conjointement depuis ces deux sites (fig. 7). La relation visuelle avec les camps de hauteur occupés au Bronze ancien, de Bracon, Cernans, Clucy et Fort Belin, est moins systématique, mais elle est également remarquable. La perception des lieux de dépôts depuis et en direction de ces points importants du paysage crée ainsi une seconde toile de relations visuelles couvrant également toute la zone centrale (fig. 8). Cette première approche permet donc de proposer l'existence d'un système fondé sur l'importance de la perceptibilité des sites dans le choix des lieux de déposition, ayant abouti à une répartition organisée des dépôts autour de la vallée de la Furieuse.

Les analyses permettent également de saisir les limites géographiques de ce système (fig. 9). En effet, plusieurs sites ne répondent pas aux mêmes critères d'emplacement. La surface visible depuis le site d'Aresches est tournée vers la haute chaîne du Jura et exclut donc totalement la zone de Salins-les-Bains. Pour les dépôts trouvés au nord du Camp du Château et du Mont Poupet (Malans, Bartherans, Chay, Cussey, Eternoz), il ne semble exister aucune relation de visibilité avec les autres groupes. Ces dépôts paraissent donc ne pas participer au phénomène remarqué dans le secteur de Salins et devaient sans doute s'implanter selon un tout autre modèle. De la même manière, les camps plus lointains d'Echay, Mesnay et Port-Lesney ont une faible emprise visuelle sur la commune actuelle de Salins et sont donc peu visibles depuis les lieux de dépôt.

La concentration des liens visuels dans le secteur central permet ainsi de proposer l'existence d'un système de communication visuelle à la transition Bronze moyen Bronze final dont le pivot serait le Camp du Château et qui fonctionnerait dans une zone limitée à une dizaine de kilomètres de diamètre (fig. 9). Profitant de la topographie particulière de ce secteur, les communautés attachées au camp fortifié auraient ainsi 
choisi les lieux servant de cadre à leurs pratiques de déposition en tenant compte de leur potentiel visuel.

Par ailleurs, il est intéressant de constater que la position des découvertes est le plus souvent en limite des zones de visibilité, que ce soit celles des sites de hauteur ou des autres dépôts. Au lieu d'être au centre de l'emprise visuelle, les dépôts sont presque systématiquement dans les premiers $5 \%$ des surfaces perceptibles (voir par ex. fig. 7). Ce phénomène s'explique par le fait qu'ils sont essentiellement placés sur des lignes de crêtes et des bords de terrasses. De la même manière, on constate que les découvertes sont pratiquement absentes du centre même de la commune de Salins. Là aussi, les choix topographiques y sont pour beaucoup puisque la vallée semble délaissée au profit des hauteurs. Les dépôts sont rejetés aux limites de la zone de $10 \mathrm{~km}$ de diamètre définie précédemment. Les lieux de déposition matérialisent donc ici la frontière de l'espace visible depuis les différents points importants du paysage et balisent ainsi un secteur dépendant visuellement du Camp du Château. À l'écart des habitats et des nécropoles, ils pourraient être considérés alors comme des marqueurs significatifs du territoire, en bordure des principales voies de pénétration du Jura centre-occidental entre la plaine de la Saône et le Plateau suisse.

Cette étude exploratoire est, bien entendu, tributaire des données archéologiques. Si les informations sur les dépôts eux-mêmes sont remarquables tant du point de vue de leur composition que de leur localisation, il n'en reste pas moins que le secteur n'a pas été entièrement prospecté et qu'il l'a surtout été dans des zones similaires. Il faudrait donc étendre les recherches à d'autres contextes topographiques et couvrir davantage la région de Salins-les-Bains. La prise en compte de la datation fine permettrait aussi de mieux appréhender les relations entre des vestiges strictement contemporains, mais également de suivre la chronologie de l'implantation des dépôts pour vérifier l'hypothèse d'une prise en compte des dépôts précédents dans l'implantation des nouveaux.

Cette étude offre néanmoins des perspectives intéressantes pour comprendre l'organisation spatiale autour du Camp du Château et les liens entre milieu naturel et pratiques culturelles. Les communautés ont marqué et chargé de mémoire le paysage qui pour elles était autant un cadre social et sans doute religieux, qu'un cadre de vie. Alors que le rôle des sanctuaires dans l'organisation et le marquage territorial est assez bien connu pour la Grèce ancienne (De Polignac 1984, Alcock et Osborne 1994), il est bien plus difficile à saisir pour l'âge du Bronze nord-alpin. Se pourrait-il qu'ici nous ayons un aperçu de ce que pouvait être un "paysage sacré » associé à un territoire contrôlé par un camp de hauteur fortifié à la fin du Bronze moyen et au début du Bronze final ? 


\section{Bibliographie}

Alcock S. et Osborne R. (éd.) (1994). Placing the Gods: Sanctuaries and Sacred Space in Ancient Greece, Oxford University Press et Clarendon Press, Oxford et New York.

BRADley R. (1990). The Passage of Arms : An Archaeological Analysis of Prehistoric Hoards and Votive Deposits, Cambridge University Press, Cambridge.

BRADLEy R. (2000). An Archaeology of Natural Places, Routledge, London et New York.

BRADLEY R. (2003). The translation of Time, in R. Van Dyke et S. Alcock (éd), Archaeologies of Memory, Blackwell, Malden (Mass), p. 221-227.

DieHM M. et FALKENSTEIN F. (2012). Die Hortfunde vom Bullenheimer Berg, in S. NOMAYO et F. FALKENSTEIN (ed.), Der Bullenheimer Berg im Fokus moderner Methoden der Archäologie, Sriftenreiche des Städtischen Museums Kitzingen, 5, Sauerbrey, Kitzingen, p. 14-18.

DIEMER G. (1995). Der Bullenheimer Berg und seine Stellung im Siedlungsgefüge der Urnenfelderkultur Mainfrankens, Materialhefte zur bayerischen Vorgeschichte, 70, M. Lassleben, Kallmünz.

DiETRICH O. (2013). A small bronze hoard from " Kronstadt-Galgenberg ". A contribution to the understanding of cultural landscapes in Middle Bronze Age southeastern Transylvania, Satu Mare - Studii şi Comunicări, nº XXIX/I, p. 167-180.

DiETRICH O. (2014). Learning from 'scrap' about Late Bronze Age hoarding practices. A biographical approach to individual acts of dedication in large metal hoards, European Journal of Archaeology, no 17.3, p. 468-486.

FISHER P.F. (1996). Extending the Applicability of Viewsheds in Landscape Planning, Photogrammetric Engineering and Remote Sensing, $\mathrm{n}^{\circ}$ 62.11, p. 1243-1306.

FONTIJN D.R. (2001-2002) [2002]. Sacrificial landscapes. Cultural Biographies of persons, objects and 'natural' places in the Bronze Age of the southern Netherlands, Analecta Praehistorica Leidensia, 33-34.

FonTIJN D. R. (2007). The significance of 'invisible' places, World Archaeology, no 39.1, p. 70-83.

GosDen C. et MARSHALl Y. (1999). The Cultural Biography of Objects, World Archaeology, $\mathrm{n}^{\mathrm{o}} 31.2$, p. 169-178.

HANSEN S. (1994). Studien zu den Metalldeponierungen während der älteren Urnenfelderzeit zwischen Rhônetal und Karpatenbecken, Universitätsforschungen zur prähistorischen Archäologie, 21, Rudolf Habelt, Bonn.

HANSEN S., NeumanN D. et VAchta T. (ed.) (2012). Hort und Raum: Aktuelle Forschungen zu bronzezeitlichen Deponierungen in Mitteleuropa, Topoi - Berlin Studies of the Ancient World, 10, De Gruyter, Berlin et Boston.

HARMANŞAH Ö. (ed.) (2014). Of Rocks and Water: Towards an Archaeology of Place, Joukowsky Institute Publication, 5, Oxbow Books, Oxford.

KOPYTOFF I. (1986). The cultural biography of things: commoditization as process, in A. ApPADURAi (éd.), The Social Life of Things, Cambridge University Press, Cambridge, p. 64-94. 
KRISTIANSEN K. (2000). Europe Before History, New Studies in Archaeology, Cambridge University Press, Cambridge.

LAKE M.W. et WOODMAN P.E. (2003). Visibility studies in archaeology : a review and case study, Environment and Planning B : Planning and Design, $\mathrm{n}^{\circ} 30.5$, p. 689-707.

MEAD G.H. (1938). The Philosophy of the Act, ed. Morris C.W. et al., University of Chicago Press, Chicago.

MiLlotte J.-P. (1963). Le Jura et les plaines de Saône aux âges des métaux, Annales littéraires de l’Université de Besançon, 59, Archéologie, 16, Les Belles Lettres, Paris.

PERSON Y. (1962). Tradition orale et chronologie, Cahiers d'Études africaines, ${ }^{\circ} 2.7$, p. 462476.

Peatfield A.A.D. (1983). The Topography of Minoan Peak Sanctuaries, Annual of the British School at Athens, $\mathrm{n}^{\circ}$ 78, p. 273-279.

PeATfield A.A.D. (1994). After the 'Big Bang' - what? Or, Minoan symbols and shrines beyond palatial collapse, in S.E. ALCOCK et R. OsBORNE (éd.), Placing the Gods : Sanctuaries and Sacred Space in Ancient Greece, Clarendon Press, Oxford, p. 19-36.

Pétrequin P., Weller O., Gauthier E. et Dufraisse A. (2001). Salt springs exploitations without pottery during Prehistory. From New Guinea to the French Jura, in PÉTREQUIN P. et BEYRIES S. (éd.), Ethno-archaeology and its transfers, Papers from a session held at the European Association of Archaeologists Fifth Annual Meeting in Bournemouth 1999, British Archaeological Reports international series, 983, Archaeopress, Oxford, p. 37-65.

PiningRe J. -F. (1998). Les dépôts de Franche-Comté : analyse spatiale, in C. MORDANT, M. PERNOT M. et V. RYCHNER (éd.), L'atelier du bronzier en Europe du XX'e au VIII siècle avant notre ère. Tome III (session de Dijon). Production, circulation et consommation du bronze, Actes du colloque international «Bronze 96 » Neuchâtel et Dijon, 1996, Éditions du Comité des Travaux historiques et scientifiques et du Centre de Recherche sur les Techniques grécoromaines de l'Université de Bourgogne, Paris et Dijon, p. 211-222.

PININGRE J.F. et GANARD V., avec la collaboration de BARRAL Ph. et BoËs É. (2004). Les nécropoles protohistoriques des Moidons et le site princier du camp du Château à Salins (Jura), Revue archéologique du Centre de la France, n 43.1, p. 292-295.

PiningRe J. -F., GABillot M., MoRdant C. et Wirth S. (à paraître). Regards inédits sur le dépôt de Larnaud dans le contexte des nouveaux dépôts découverts dans le Jura français, in Choice Pieces. The destruction and manipulation of goods in the Later Bronze Age : from reuse to sacrifice, International conference Rome, 16-18 February 2012.

PiningRe J. F. et GRUT H. (2009). Dépôts et lieux de déposition de bronzes dans la région salinoise (Jura) au XV $\mathrm{XV}^{\mathrm{e}}$-XIV ${ }^{\mathrm{e}}$ siècle av. J.-C., in A. RICHARD, Ph. BARRAL et A. DAUBIGNEY (dir.), L'isthme européen Rhin-Saône-Rhône dans la Protohistoire. Approches nouvelles en hommage à Jacques-Pierre MiLLOTTE, actes du colloque de Besançon, 17-18 octobre 2006, Annales littéraires de l'Université de Franche-Comté, 860, Série "Environnement, sociétés et archéologie », 13, Presses universitaires de Franche-Comté, Besançon, p. 183199.

Polignac F. (de) (1984). La naissance de la cité grecque. Cultes, espace et société, VIII ${ }^{e}-$ VII siècles avant J.-C., La Découverte, Paris.

ROWLANDS M. (1993). The role of memory in the transmission of culture, World Archaeology, $\mathrm{n}^{\circ}$ 25.2, p. 141-51. 
SCHAMA S. (1996). Landscape and Memory, Vintage Books, New York.

SOETENS S., DriesSEN J., SARRIS S. et TOPOUZI S. (2002). The Minoan peak sanctuary landscape through a GIS, Archeologia e Calcolatori, nº 13, p. 161-170.

SOROCEANU T. (1995). Die Fundumstände bronzezeitlicher Deponierungen - Ein Beitrag zur Hortdeutung beiderseits der Karpaten, in SOROCEANU T. (dir.), Bronzefunde aus Rumänien, Prähistorische Archäologie in Südosteuropa, 10, V. Spiess, Berlin, p. 15-80.

SOROCEANU T. (2012). Die Fundplätze bronzezeitlicher Horte im heutigen Rumänien, in S. HANSEN, D. NeumanN et T. VAchta (éd.), Hort und Raum: Aktuelle Forschungen zu bronzezeitlichen Deponierungen in Mitteleuropa, Topoi - Berlin Studies of the Ancient World, 10, De Gruyter, Berlin et Boston, p. 227-255.

STEAD I. M. (1998). The Salisbury Hoard, Tempus, Stroud.

VAN DYKe R. et AlCOCK S. (2003). Archaeologies of Memory : An Introduction, in R. Van DYKE et S. AlCOCK (ed.), Archaeologies of Memory, Blackwell, Malden (Mass), p. 1-13.

WheAtLey D. et GILLINGS M. (2000). Vision, perception and GIS : developing enriched approaches to the study of archaeological visibility, in G. LOCK (ed.), Beyond the Map: Archaeology and Spatial Technologies, IOS Press, NATO Science Series, Series A, Life sciences, 321, Amsterdam, p. 1-27.

WindHOLZ-KONRAD M. (2002). Ein späturnenfelderzeitlicher Depotfund von der Rettenwand in Kapfenberg, Steiermark, Fundberichte aus Österreich, nº 41, p. 407-424.

WindHOlZ-KONRAD M. (2005). Die Rabenwand - ein neuer prähistorischer Depotfundplatz im Ausseerland, Steiermark, Fundberichte aus Österreich, no 43, p. 289-349.

WindHOLZ-KONRAD M. (2010). Der prähistorische Depotfund vom Brandgraben im Kainischtal, Bad Aussee: mit einem Corpus der urnenfelderzeitlichen Mehrstückhorte zwischen Öden- und Hallstättersee. Thèse de doctorat. Institut für Archäologie, Karl-FranzensUniversität, Graz. 


\section{Illustrations}

Figure 1: Environnement géographique du site de Salins-les-Bains. Au centre, l'éperon du Camp du Château et le village de Pretin; en arrière-plan, le Mont-Poupet (cliché J. Aubert).

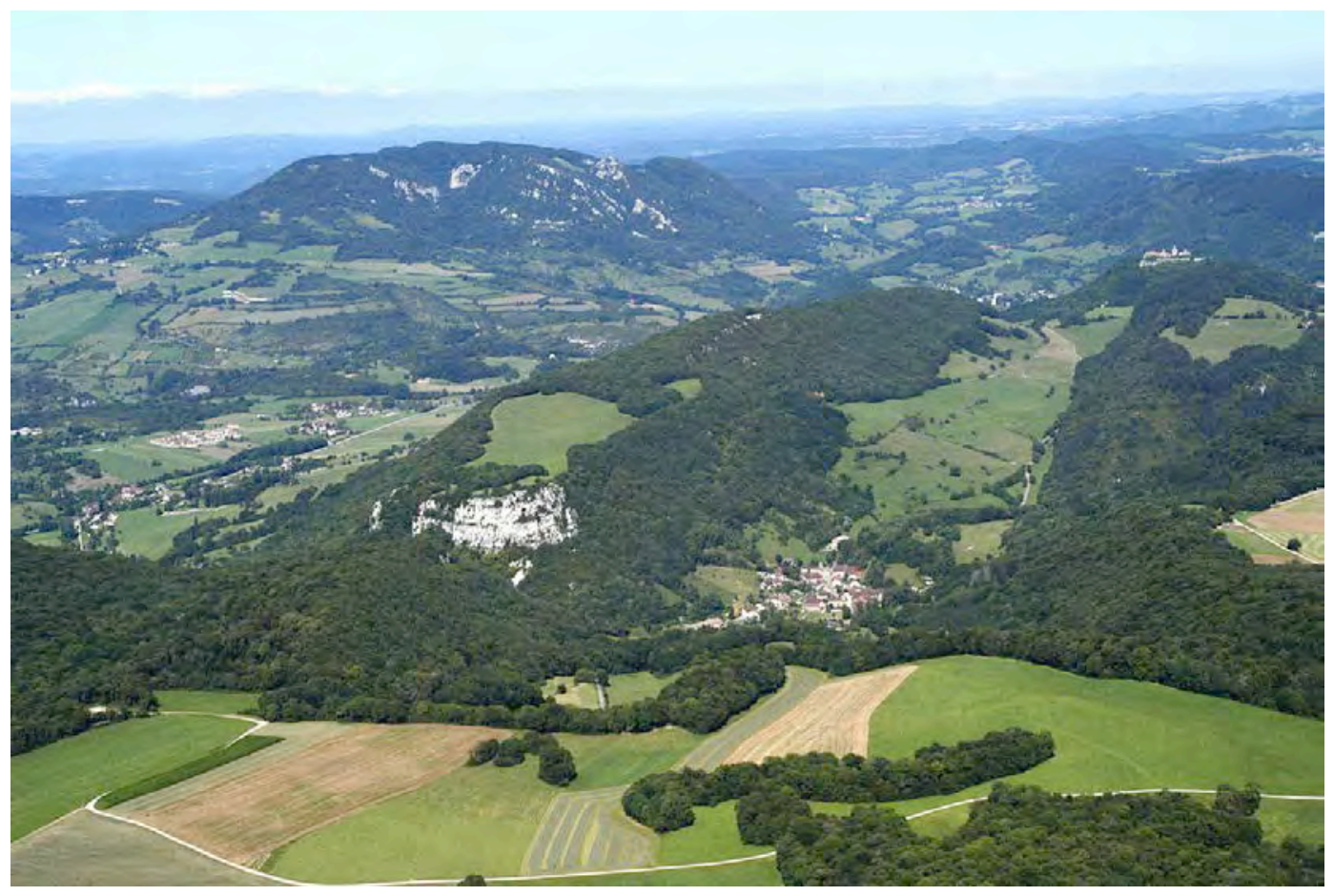


Figure 2 : Carte de répartition des dépôts de la région salinoise (CAO J.-F. Piningre)

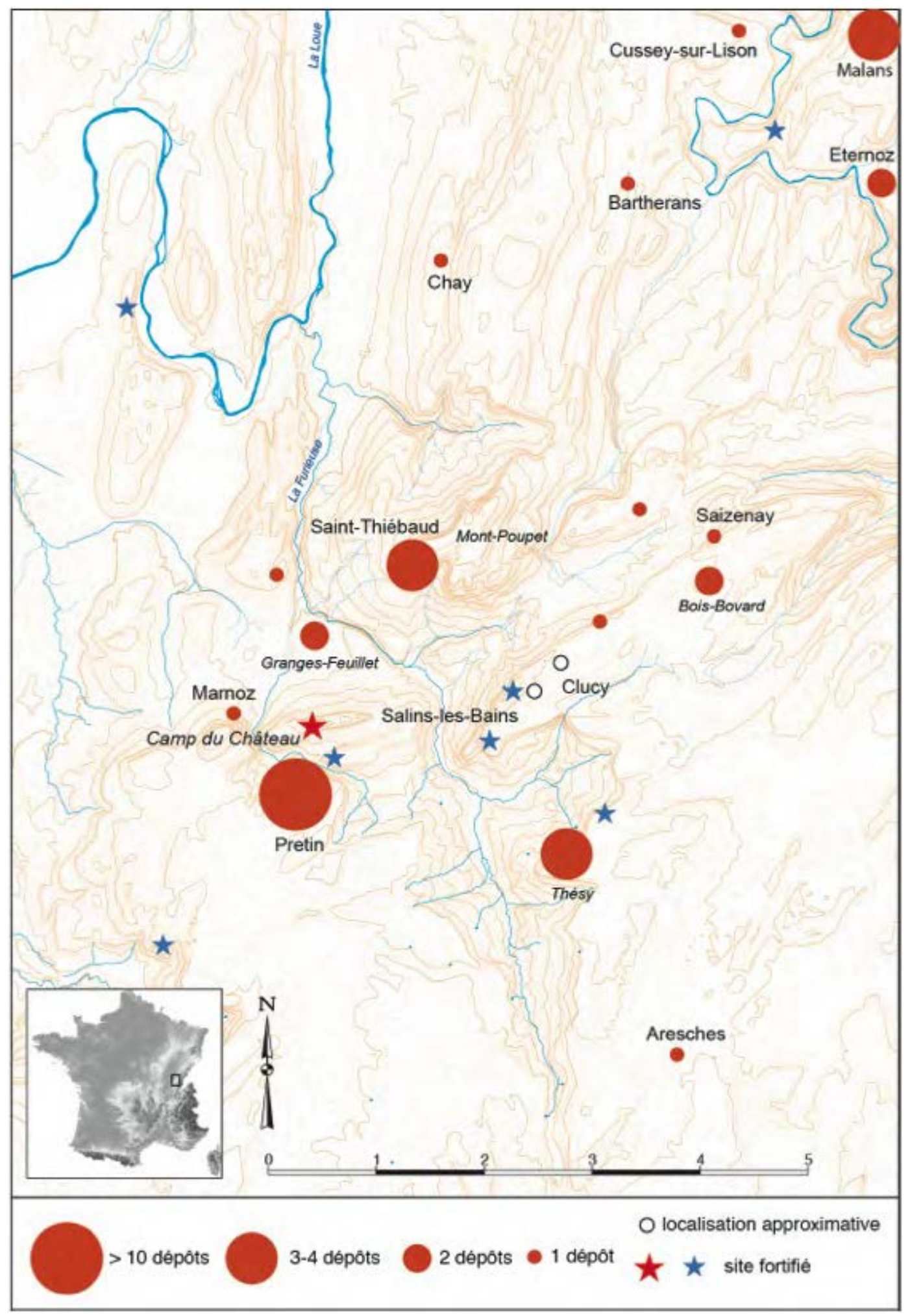


Figure 3 : Diagramme du poids des lingots et des objets des dépôts de la région salinoise (DAO J.-F. Piningre).

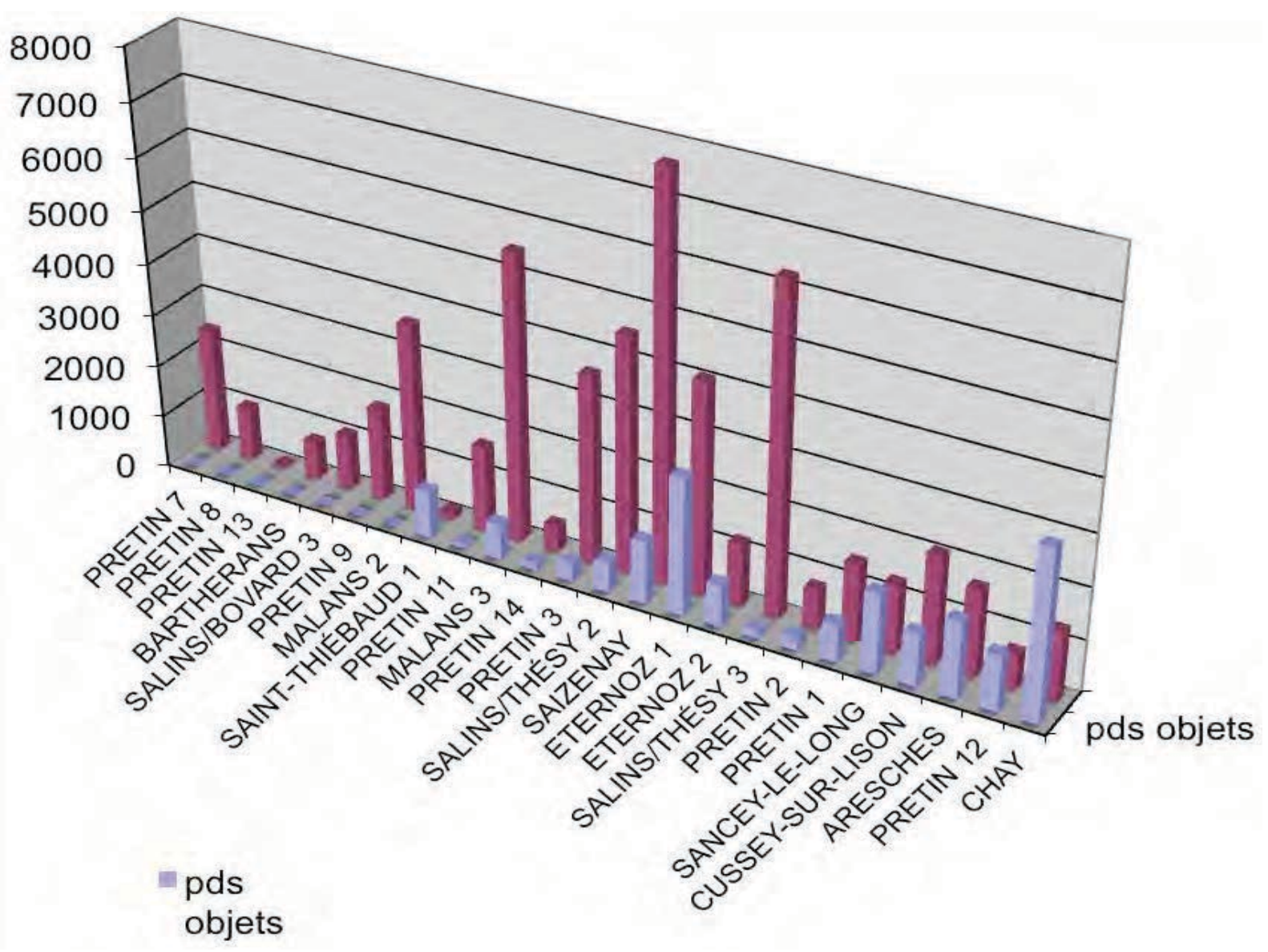


Figure 4: Représentation chronologique des dépôts de la région salinoise (DAO J.-F. Piningre).

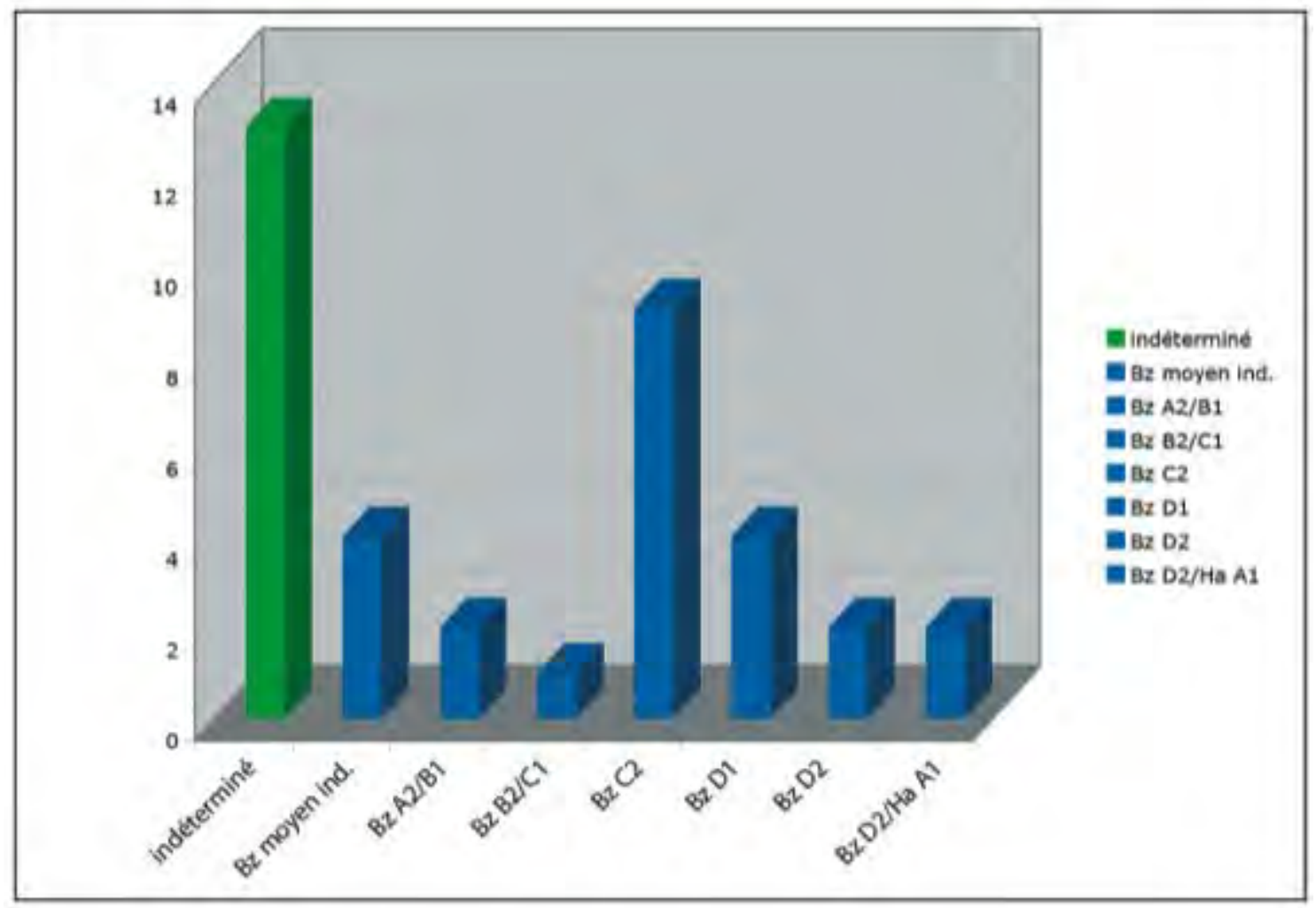

Figure 5 : Visibilité depuis (a) et en direction (b) de chacun des dépôts de bronzes et des sites de hauteur (DAO E. Gauthier).
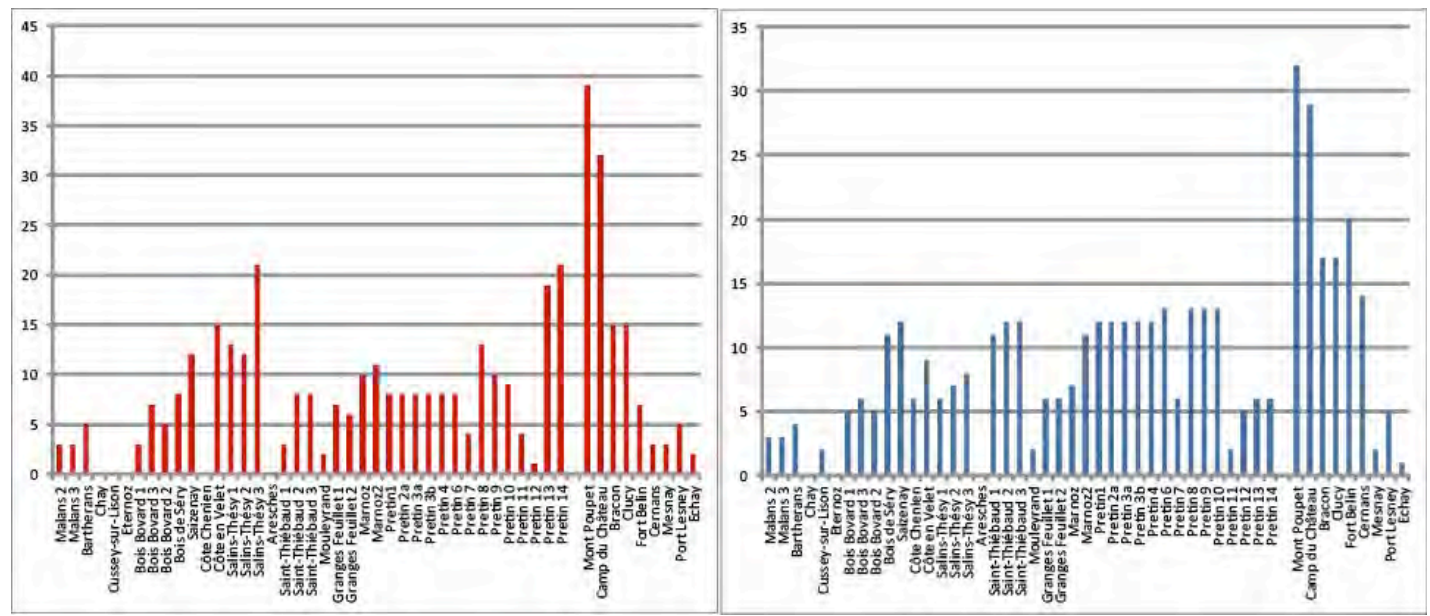
Figure 6 : Relations de perception entre les dépôts (Fond IGN BD ALTI; SIG et CAO E. Gauthier)
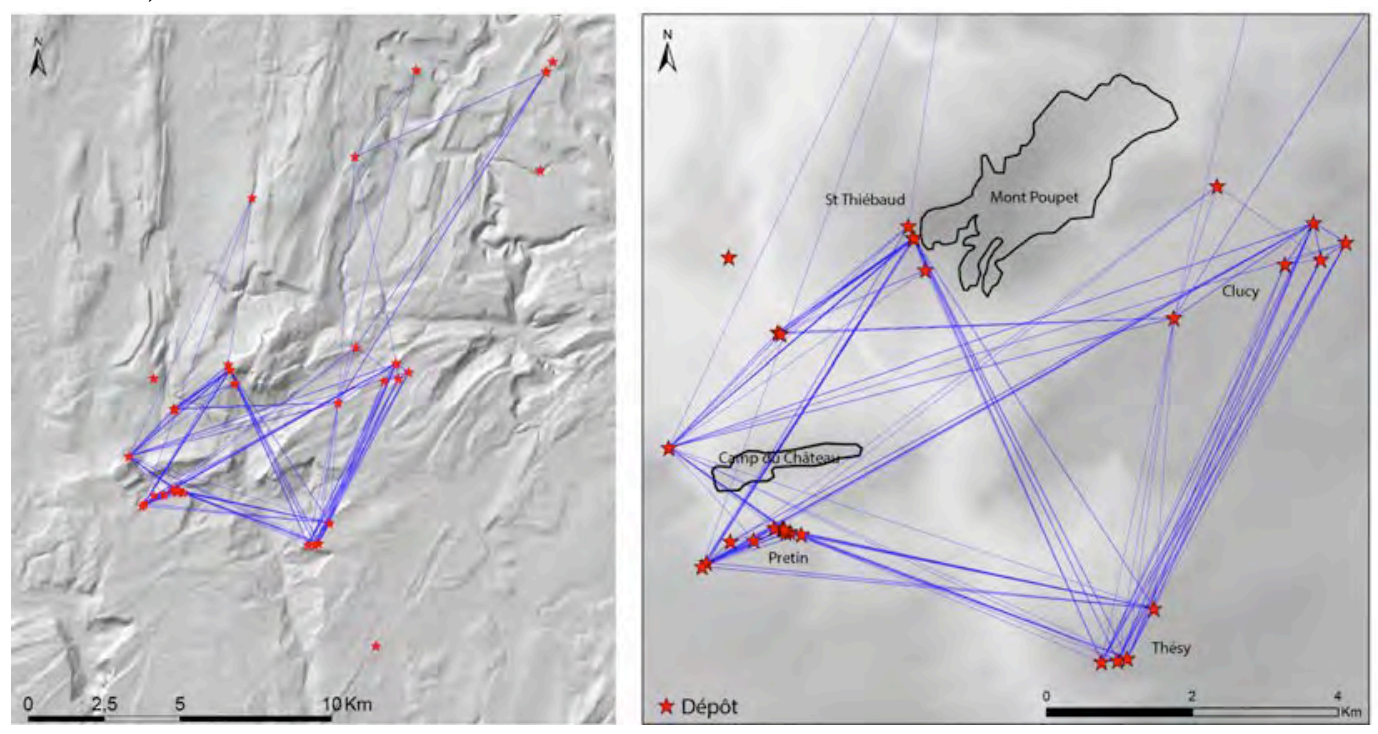

Figure 7 : Localisation des dépôts dans les zones de visibilité cumulée du Mont Poupet et du Camp du Château (Fond IGN BD ALTI et Route 120 ; SIG et CAO E. Gauthier)

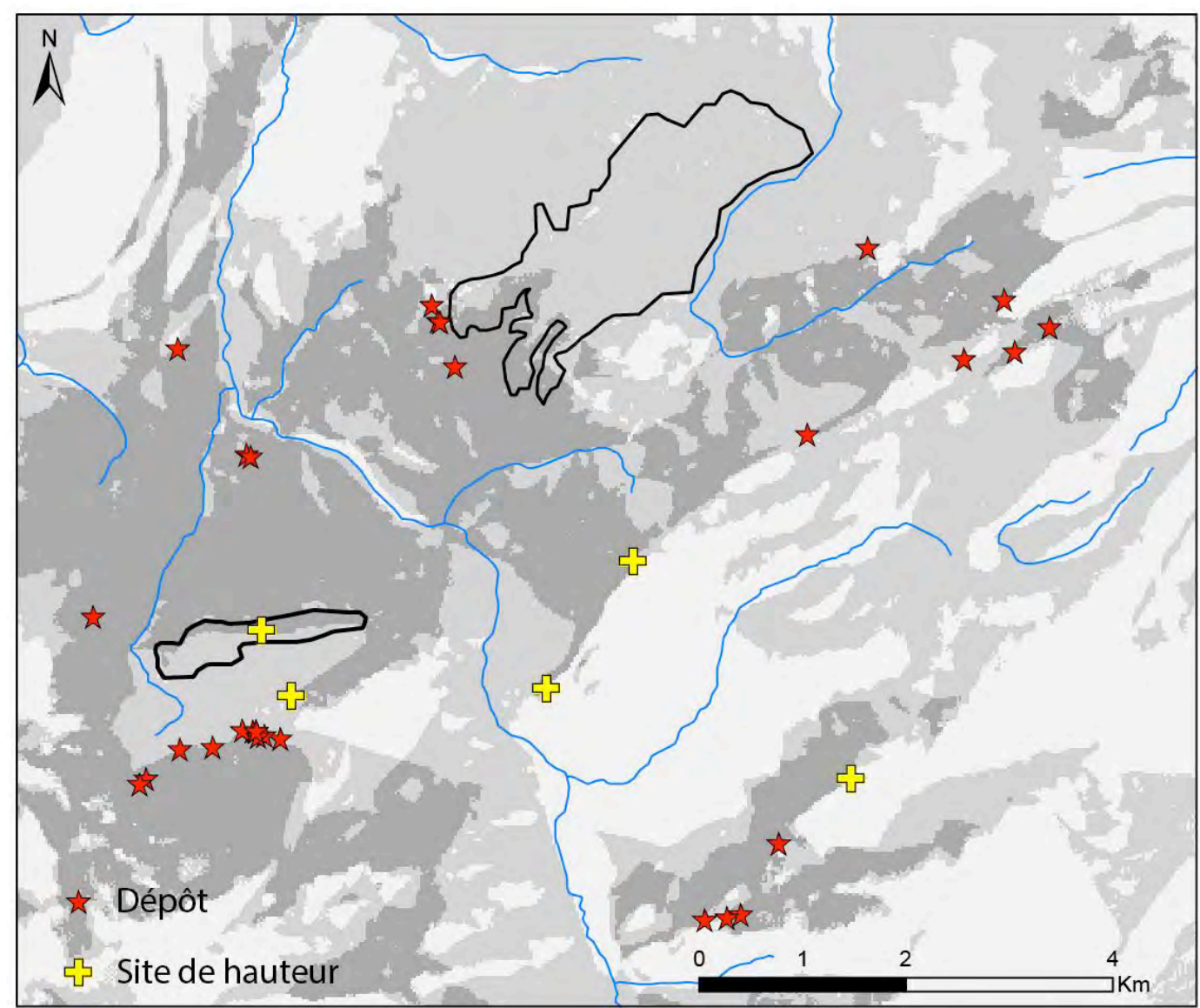


Figure 8 : Relations de perception entre les dépôts et les sites de hauteur (Fond IGN BD ALTI ; SIG et CAO E. Gauthier)
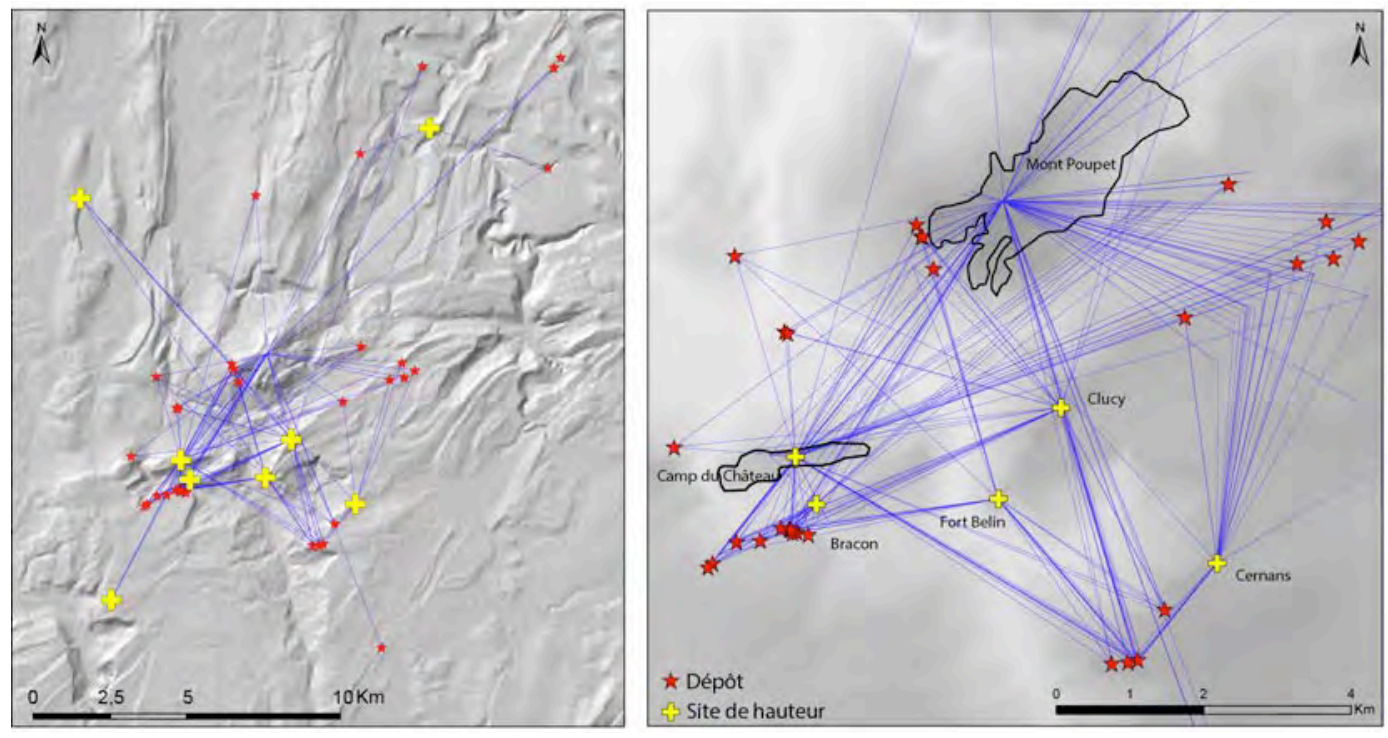
Figure 9: Modélisation du système de communication visuelle associé au Camp du Château (Fond IGN BD ALTI et Route 120, SIG et CAO E. Gauthier).

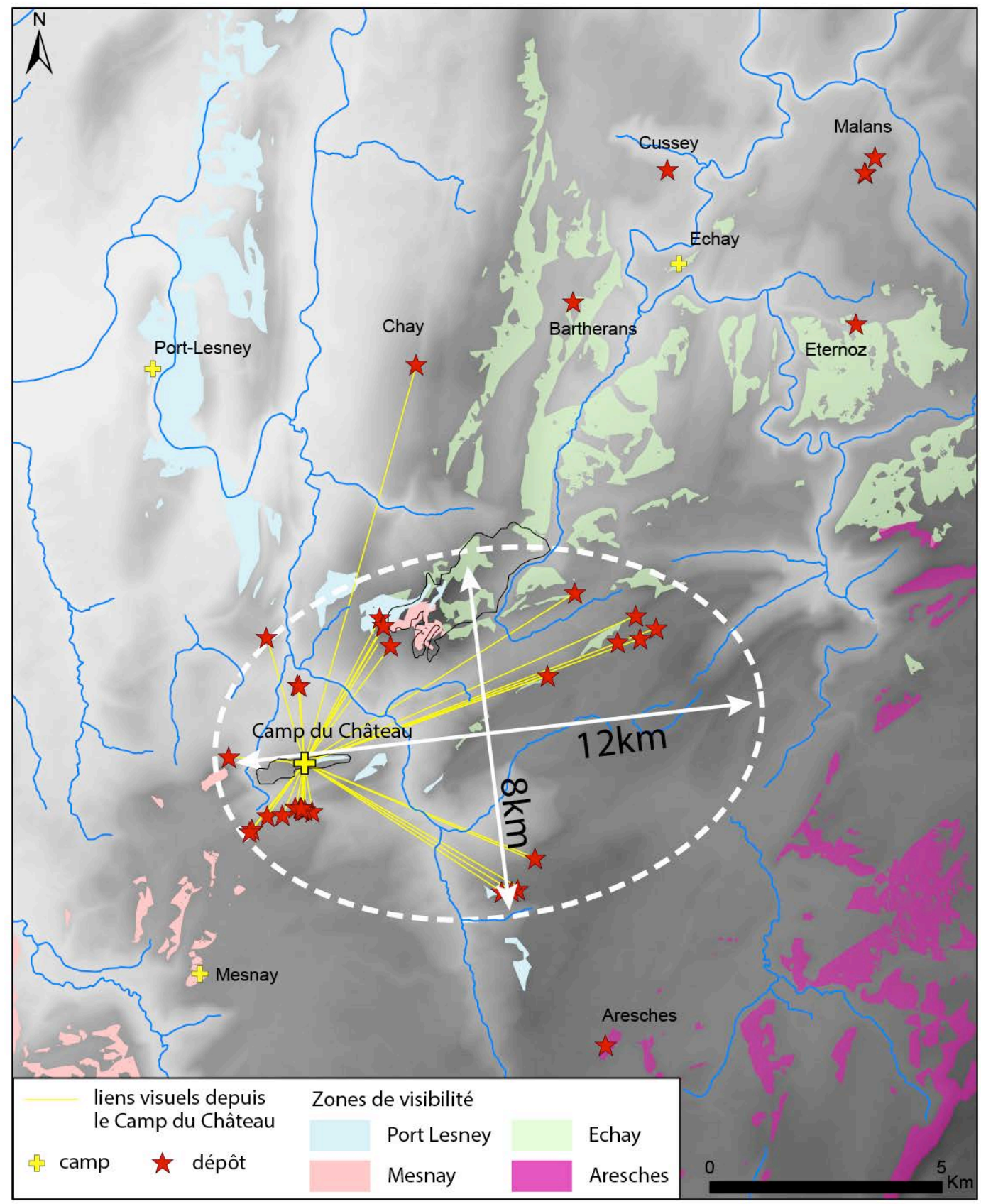




\title{
Expression graphique et espace de communication à l'âge du Bronze final. Le bassin rhénan et les Alpes occidentales (du XIII ${ }^{e}$ au IX $X^{e}$ siècle av. J.-C.)
}

\author{
Vincent GEORGES \\ Inrap Auvergne Rhône-Alpes UMR 6298 Artehis, Dijon
}

\begin{abstract}
Extrait de : Olivier BuchsenschutZ, Christian Jeunesse, Claude Mordant et Denis Vialou (dir.), Signes et communication dans les civilisations de la parole, Paris, Édition électronique du CTHS (Actes des congrès des sociétés historiques et scientifiques), 2015.

Cet article a été validé par le comité de lecture des Éditions du CTHS dans le cadre de la publication des actes du $139^{\mathrm{e}}$ Congrès national des sociétés historiques et scientifiques tenu à Nîmes en 2014.
\end{abstract}

\begin{abstract}
Résumé
$\mathrm{Du} \mathrm{XIII}{ }^{\mathrm{e}}$ au IX ${ }^{\mathrm{e}}$ siècle av. J.-C., les décors portés sur les bracelets alpins et rhénans sont sujets à d'importantes transformations. Dans cet article, ces changements seront lus comme une influence des élites à l'origine de ces variations au sein de sociétés segmentaires fondées sur les liens de parenté. Sur métal ou textile, ces graphismes complexes seront perçus et interprétés selon une lecture et une hypothèse de la transmission du pouvoir par voie utérine. Dans ce cadre, l'ordre successoral pourrait se dérouler sur trois générations avec une mère appartenant à la classe intermédiaire dont la descendance devrait pérenniser l'autorité de sa lignée. En tout état de cause, le rôle de certaines femmes aboutirait au perfectionnement de leur apparat sur le long terme et les « sémioses graphiques » utilisées tiraillées entre innovations et traditions se déploieraient au gré des échanges matrimoniaux de haut rang.
\end{abstract}

Mots-clés: arts graphiques ; bracelets ; parures annulaires ; parenté ; élites ; échanges matrimoniaux.

Key words: graphic arts ; bracelets ; annular jewellery ; kinship ; elites ; matrimonial exchanges.

\begin{abstract}
Between the 13th and the 9th century BC, the motifs on bracelets the Alps and Rhineland undergo important changes. In this paper, these changes will be considered as influenced by the elite within a divided society built upon the foundations of kinship. Drawn on metal or on textiles, these complex motifs are perceived and interpreted as the transmission of power via the female line. The successoral order could play out over three generations with a mother belonging to the intermediary class whose descendants seek to perpetuate their lineage. In any case, the role of some women will result in the long term improvement of their status and the graphic semiosis in place will be torn between innovation and tradition within a system of high ranking matrimonial exchanges. (Traduction Rebecca Peake)
\end{abstract}

Le souci de l'apparat au sein des sociétés européennes du Bronze final se manifeste en particulier par des ornementations complexes sur les bracelets en bronze. La mise à plat orthogonale de ces graphismes constitue l'étape préalable pour une ordination dans des arbres stylistiques (A.S.) (Georges, à paraître). La chronologie relative établie entre certaines occurrences est décisive pour dévoiler des tendances évolutives dominantes tout en pouvant faire référence à la chronologie absolue des périodes concernées. La lecture spatiale des arbres stylistiques donne aussi accès aux mutations perçues au sein des aires productrices ou réceptrices de ces graphismes. Le rôle de ces décors dans les organisations sociales peut alors être discuté grâce aux occurrences rhéno-alpines qui se comptent par plusieurs centaines. 


\section{Des tendances évolutives et des aires culturelles}

Des styles variés propres au Bronze final se déploient du bassin rhénan jusqu'aux Alpes méridionales (fig. 1). Ces représentations graphiques sont liées par une même racine cognitive. L'un des arbres est commun aux bracelets des types Cortaillod et Corcelettes et il bénéficie de bons repères chronologiques: sa branche initiale est caractéristique du début du Bronze moyen nord-alpin occidental (fig. 2, $\mathrm{n}^{\circ} 1$ ); les branches 2 à 24 s'échelonnent du Bronze moyen jusqu'aux décors du type Pourrières propres au Hallstatt A2 (Pászthory 1985, p. 149-152). Les types Cortaillod et Corcelettes figurent au niveau des ramures 25-34 et 35-57. Les premiers sont présents à la période du Hallstatt B1 ancien et les seconds au Ha B2, vers 950 av. J.-C. (ibid. 1985, p. 152-157 et 186-200; Rychner et al. 1996, p. 307 et suiv.).

Les ocelles complets sont d'abord seuls (fig. 2, 3-32) puis les branches 34 à 38 montrent les mêmes désormais reliés par des rayons sur des bracelets de grands formats avec de nouvelles extrémités distales; ces spécimens conservent de fortes analogies avec le style décoratif antérieur. Après 950 av. J.-C., de petits ocelles équidistants reliés par des rayons étroits figurent sur des bracelets réduits en taille et calibrés, ils sont spécifiques de la rive nord du lac de Neuchâtel (fig. 2, 40-42). L'ordonnancement se densifie à outrance au tournant $\mathrm{du} \mathrm{IX}^{\mathrm{e}}$ siècle (fig. 2, 44-48), avant de dériver vers des organisations plus sommaires (fig. 2, 49-57) sur une vaste aire nord-alpine avec des développements au Premier âge du Fer (Pászthory 1985 ; Véber 2009 ; Siepen 2005 ; Schaeffer 1926 ; Dunning et Rychner 1994, p. 63 et suiv. ; Reim 1994, p. 99 et suiv.).

Contemporain du type Corcelettes et de même morphologie, le type Auvernier s'en distingue en agrégeant des hachures obliques en position centrale (fig. 3, 3-10) (Pászthory 1985, p. 181-185). L'ouest des Alpes montre cet usage dès le début du Bronze final (fig. 3, 11-15), alors qu'au nord et au nord-est, les hachures couvrent la totalité des joncs, ou presque, comme à Aislingen au Ha A1 (fig. 3, 16). Apparu au Bronze moyen du côté de la Mer Baltique (Schubart 1972), ce décor progresse ensuite vers le sud.

Au Nord des Alpes, l'épicentre de la famille des bracelets dits réniformes occupe la région du Rhin moyen (fig. 4, 8-30); ils font l'objet de mutations graphiques du Ha A2 au Ha B1 (Beck 1980, pl. 3 et 53 ; Richter 1970, pl. 35). Auparavant, au début du Bronze final, un bracelet non encore réniforme mais déjà côtelé figure dans le dépôt de Praha-Bubeneč 2 (fig. 4,7). Son décor fait référence à la même racine cognitive qu'il partage avec l'un des décors de bracelets de la dame de Mathay au début du Ha A2 (Piningre 2014, p. 399 et suiv.) (fig. 4, 7bis). D'autres dessins orientaux en contextes plus précoces (Bronze C et D) s'y rattachent aussi (fig. 4, 1-6).

Les bracelets réniformes s'affirment en taille et en nombre d'ocelles au cours du Ha A2. Les décors à chevrons centraux avec des lignes de pointillés sont typiques du Rhin moyen (fig. 4, 10-19). Au Ha B1, sur une aire élargie, des ocelles axiaux émergent avec l'écartement des chevrons (fig. 4, 20-21 et 20-30). Il s'ensuit des dispositifs simplifiés avec des chevrons axiaux affrontés (fig. 4,31-41). Les pointillés ceinturent désormais les ocelles (fig. 4, 32 et 39-41). Au cours du Ha B1, à compter du $x^{\mathrm{e}}$ siècle, des assemblages en blocs juxtaposés (fig. 1, 5 ; fig. 4, 42-43) pénètrent jusque dans les Alpes internes (fig. 4, n 42 43 ; fig. 5A) et ornent aussi des supports non réniformes (Pászthory 1985, pl. 62 et 63).

La gamme des décors des réniformes rhénans contraste régionalement avec ceux des jambières à spirales (Kubach 1991, p. 141 et suiv.) (fig. 1, 2) ; le décor réniforme de Blanot (fig. 4, 25) tranche tout autant au Ha B1 avec ceux des jambières de ce même dépôt typique de la France orientale et reprenant aussi des registres ornementaux locaux plus anciens. Les riches compositions des réniformes se diffusent largement au Ha B1 (fig. 5A). L'extension et l'intensité sont supérieures à la dispersion au Ha A1, du style Poype parti du sud-est de la France (Georges, à paraître, fig. 11). 
À la fin $\mathrm{du} \mathrm{XI} \mathrm{I}^{\mathrm{e}}$ siècle ou au début du $\mathrm{X}^{\mathrm{e}}$ siècle (Ha A2/Ha B1), le dépôt de Larnaud occupe une position chronologique intermédiaire (Millotte 1993, p. 35-52). Si les décors réniformes complexes du Ha $\mathrm{A} 2$ sont absents, un jonc simple orné fait tout de même écho aux ornements orientaux plus précoces (fig. 4, 8) ; le style pré-Cortaillod ancien du Ha A2 y est bien présent (fig. 2, 21). Larnaud documente aussi le type Zerba et le «style Helston » avec des spécimens déjà en circulation vers 1000 av. J.-C. (fig. 5B, 9-10 et 13-14 et 5C, 6-7 et 18-19). La constitution de ce dépôt peut faire suite à des démantèlements de paires de bracelets avec ou sans bris rituels, lors de leur soustraction au moins partielle à la future parure funéraire des défunts. Le recyclage massif de pièces endommagées se fait sur de longues distances mais aussi, là où les bracelets sont portés. Des enfouissements volontaires en dehors des tombes peuvent participer comme nous le verrons plus loin, d'un retraitement non économique.

Ainsi, pour chaque arbre stylistique, un espace initial à l'ouest de la Suisse (fig. 2, 1-22) ou en Bavière oriental et en Bohême (fig. 4, 1-7) semble impulser des gammes décoratives qui seront ultérieurement transférées vers d'autres régions réceptrices. L'interpolation des analogies stylistiques et des données typo-chronologiques permet de suivre les tendances sur la durée en intégrant des bracelets traditionnellement donnés jusqu'ici comme mal datés.

\section{Sémiose graphique et identités féminines}

Avant leur déchéance post-mortem, des parures ont vocation à signaler certaines femmes des communautés de l'âge du Bronze. Dans notre hypothèse, les arbres stylistiques retraceraient l'historique des gammes ornementales employées par les artisans bronziers. Des rétroactions complexes entre affichage ostentatoire et identité communautaire seraient à l'origine de la complexification des styles à partir d'une racine cognitive initiale. Cette forme de communication composée de signes est non verbale ( $c f$. Fontanille et Beyaert-Geslin 2006, p. 1071 et suiv.) et caractéristique d'un groupe et d'une période tout en correspondant à une construction dans la durée ; c'est ce que nous proposons de nommer « sémiose graphique ».

Il est communément accepté que les sociétés protohistoriques européennes dites segmentaires, partagées et regroupées en lignées, reposent sur l'exogamie avec des réciprocités donnant lieu aux échanges matrimoniaux; le degré de cette réciprocité conditionne un niveau variable d'endogamie (Rowlands 1998, p. 142 et suiv.).

Si l'on accepte ces prémisses, il est permis d'envisager que la pratique ostentatoire des graphismes soit liée à des considérations matrimoniales (fig. 5A). Au cours de son développement, la sémiose graphique est sujette à une surcharge des décors ou à leur déconstruction rapide.

\section{L'incorporation des mythogrammes des parures métalliques en relation avec d'autres supports, textiles en particulier}

Certains styles incorporent des mythogrammes définis ici en tant que représentations aux contenus mythologiques (Leroi-Gourhan 1964, p. 278-283). Des bracelets rhénans, génois et italiques déclinent cette intégration (fig. 6, 1-10, 12-25; 26-32) avec, à la fin du Bronze final des silhouettes aviformes (fig. 6, 23-25) également recensées sur les céramiques du Bronze final (Gomez de Soto 1993). On retrouve ce type d'image possédant une dimension cosmogonique dans les figurations des barques solaires sur récipients métalliques (Kaul 1998, fig. 170, voir aussi Wirth 2006, p. 331 et suiv.). 
Un décor textile d'une tombe du Latium témoigne au $\mathrm{X}^{\mathrm{e}}$ siècle av. J.-C. d'une possible correspondance avec les décors contemporains des bracelets du Nord de la péninsule (fig. 7 ; fig. 5C : 4-5, 10-17, et 23-24). La technique textile mise en œuvre est celle du métier à lattes adopté au Bronze final. Auparavant, dans les Terramares, l'important corpus des pièces manufacturées en matières dures organiques est dépourvu des tablettes spécifiques de ces métiers, mais ces habitats ont livré le bâton cylindrique de Castione dei Marchesi (Eberschweiler 1998, fig. 9). Cet objet possède les caractéristiques nécessaires pour servir de montant de métier à broder (Georges 2007 : p. 307 et fig. 161-162), à l'instar des exemplaires suisses très comparables, mais plus tardifs de Zürich et Mörigen (ibid., fig. 5 et 7). Nonobstant l'interprétation qui en été faite (Cardarelli et al. 2001), ces bâtons ne sauraient être des fléaux de balances; nous insistons ici sur l'absence de perforation centrale ou de tout autre moyen de fixation nécessaire à l'accrochage du ruban de suspension axiale sur l'instrument composite. Les compositions géométriques complexes de ces bâtons sont partagées avec celles des décors de bracelets alpins du fait de leur origine vestimentaire (Georges, à paraître, fig. 16).

Le textile possède une valeur centrale dans le costume et il influence les supports annexes qui l'environnent. Certains tintinnabuli féminins du Bronze final vont jusqu'à représenter des métiers à tisser (Fath 2010, p. 254 et suiv.). Le décor villanovien du «trône » de Verruchio glorifie lui aussi la pratique féminine du tissage en faisant usage des mythogrammes cosmogoniques aviformes précités (Verger 2010, p. 66-73). Au Bronze final, les figurines féminines d'Europe centrale recensées par G. Schumacher-Matthaüs en 1985 s'inscrivent dans ce contexte global en étant ornées des mêmes méandres et ocelles que les bracelets réniformes (fig. 6, 1-10) et les surfaces discoïdes mycéniennes ou d'Europe intérieure (Gimbutas 1965, p. 63, fig. 27 ; Bouzek 2011, p. 43, fig. 35b). Ces symboles cosmogoniques sont étroitement liés à un discours religieux relayé et incarné par des responsabilités pontificales réservées aux élites de l'âge du Fer et plus largement indo-européennes (Scheid 2005 ; Renou 2004).

\section{L'apport du paradigme de la transmission du pouvoir par voie utérine}

L'onomastique étrusque fait état de grandes dames nommées à la fois par leur lignée d'origine et celle de leur mari (Briquel 1993, p. 120). Ces alliances matrimoniales sont le fait de la parenté indifférenciée en usage en Europe occidentale : cette parenté cognatique se superpose à la filiation. Le Latium montre que cette parenté est favorable à un pouvoir transmis par voie utérine dans le cadre d'une union nuptiale contractée à cet effet. À l'époque de Romulus, les gens patriarcales usent de cette pratique décrite pour d'autres peuples de l'Antiquité classique. Le pouvoir de l'homme se transmet à un tiers par l'intermédiaire d'une mère qui n'est pas son épouse. Si ni le fils, ni la fille, ne peuvent hériter du pouvoir paternel, ils peuvent donc le cas échéant, en être les dépositaires pour le compte de leur lignée et de leur descendance directe (Camous 2010, p. 288-292 ; Frazer 1981, p. 413).

Les mariages afférents, en bru, en gendre ou encore ceux de neveux et de nièces, nécessitent de déployer les stratégies adéquates pour la reproduction des élites. La transmission sur trois générations laisse du temps pour préparer la succession, soit de procéder à l'alliance jugée la plus appropriée. La faible densité structurelle des élites concourt aussi à procéder aux échanges matrimoniaux sur une base monogame. Les élites européennes et eurasiatiques de la Protohistoire ancienne se seraient distinguées par des unions isogames (Barry et al. 2000, p. 727) à la tête de lignées dans le contexte d'un échange généralisé (Rowlands 1998, p. 142-182 ; cf. Granet 1939, partie 3, chapitre 3).

La sélection des progénitures des frères et sœurs pour les mariages successoraux des lignées est de nature à renforcer l'unité du groupe au moment de l'alliance avec une lignée tierce. Les mariages obliques entre deux générations peuvent aussi survenir par 
choix comme par défaut. Pour l'Europe intérieure, au $\mathrm{VI}^{\mathrm{e}}$ siècle av. J.-C., la référence indirecte de Tite-Live à la mère des neveux du roi biturige Ambigat est une source rare et précieuse pour poser l'éventualité d'une transmission du pouvoir par voie utérine (Milcent 2004, p. 209). Les deux neveux sont appelés à exercer les plus hautes fonctions militaires par délégation directe, soit de la part de leur oncle (Baillet 1989, Tite-Live, Livre $V$, 34). La filiation agnatique, de père en fils émergerait dès l'âge du Fer sous l'effet du droit foncier selon divers témoignages ethnographiques et gréco-romains (Camous 2010, p. 292 ; Leduc 1991, p. 259 et suiv. ; Todd 2011, p. 331-345).

L'hypothèse d'une parenté cognatique avec une transmission du pouvoir par voie utérine exposée plus haut mérite d'être formulée pour l'âge du Bronze européen, non plus en faisant uniquement référence à un cadre théorique déterminé par des écrits anciens, mais en s'appuyant sur des faits matériels compatibles ou pouvant suggérer des alternatives. La sémiose graphique des bracelets pourrait nous fournir une matière utile en prise directe avec la parenté exogamique dans les différents espaces de communication où celle-ci s'exprime (fig. 5A).

La figure 8 explore les cas où un chef emploie sa descendance directe pour transmettre son pouvoir par une autre épouse que la sienne (fille ou bru). Un décalage générationnel intervient entre des parures de très haut rang, masculines et féminines, et ne pouvant pas être partagées entre époux, d'où la rareté des ensembles archéologiques afférents présentant à la fois des épées et des bracelets sur lesquels nous aurons à revenir. L'hypothèse repose sur un ordre proto-institutionnel établissant la transmission du pouvoir par voie utérine.

Le cas $n^{\circ} 1$ fait référence à un mariage en bru et le second à un mariage en gendre. En cas de procédures interchangeables, la lignée bénéficie au gré des circonstances d'un éventail de choix supplémentaires. Cet étalement sur trois générations accroît aussi le nombre des possibilités tout en augmentant l'éventualité d'une régence. Sans cet accroc successoral, le mode transitoire normal rend les deux premières générations solidaires du devenir de la lignée.

La dame de Blanot de l'âge du Bronze final s'accorderait davantage avec l'option d'un mariage en gendre, $\mathrm{du}$ fait des petites jambières présumées exprimer, selon une thèse séduisante, sa petite enfance (Verger 1998, p. 33 et suiv.). Ce scénario écarte le fait qu'il puisse s'agir de parures de bras avec un usage simultané d'un même décor pour les membres supérieurs et inférieurs. L'observation des 54 décors de Vinols du Bronze moyen, $150 \mathrm{~km}$ au sud de Blanot, amène justement à proposer ce port de doubles paires de parures annulaires dans cette région du Massif central oriental. L'association des objets dans ce dépôt implique très probablement une expression communautaire. Les groupes de décors ont pu suggérer des alliances matrimoniales intracommunautaires de haut rang entre lignées parallèles (Georges 2007, fig. 174). L'endogamie vraie n'aurait pas touché les autres unions du groupe. Le renversement de cette tendance endogamique des élites au Bronze indiquerait une recrudescence de l'exogamie avec des alliances internes aux groupes devenues moins cruciales i.e. supplantées par des alliances externes entre des lignées parvenues à atteindre un statut supérieur à l'intérieur de leur groupe d'origine.

Quoi qu'il en soit, le caractère ostentatoire des sémioses graphiques se placerait dans ce processus de parenté associé à l'exercice du pouvoir. La dimension intergénérationnelle expliquerait le succès au long cours marqué par un certain conservatisme.

\section{Le passage de souveraineté: intronisation par l'échange et la relégation des prestations matrimoniales}

Avec ou sans l'existence du «pouvoir utérin » et dans des contextes exogamiques, la 
parenté des élites de l'âge du Bronze conduit sans nul doute à l'éclosion de «maisons » regroupant des individus et constitutives des personnes morales liées à des territoires selon la définition donnée par C. Lévi-Strauss (Lévi-Strauss 1984, p. 190, voir aussi concernant le caractère ubiquiste de ce mode d'organisation, Lévi-Strauss 2008, La voie des masques). Les identités graphiques enregistrées sur les bracelets correspondraient à différents modèles institutionnels dont elles seraient l'incarnation, de sorte que des maisons puissent partager des identités graphiques extrêmement proches voire identiques. On ignore évidemment si la maison en tant que telle se distingue du lignage à l'âge du Bronze. Seul compte le fait que l'ordre successoral mobilise des règles, tantôt dogmatiques, tantôt astucieusement amendées au bénéfice d'un lignage basique ou d'une maison princière.

À la disparition des chefs à épée et des détentrices de bracelets richement ornés, le métal manufacturé perd sa raison d'être, et des anciennes prestations matrimoniales peuvent être reléguées, soit quitter le champ des sépultures et des échanges (Meillassoux 2008, p. 97 et suiv. ; Verger 1992, p. 135 et suiv. ; Fischer 2012 ; Bradley 1990, p. 40). Ce dernier auteur cite C. Meillassoux concernant l'exclusion définitive des échanges de certaines richesses une fois que la mort a rompu une alliance matrimoniale, mais aussi G. Dupré et P.-P. Rey (1969) qui insistent sur la forte déperdition des surplus agropastoraux pour fluidifier les échanges matrimoniaux entre des élites de plus en plus détachées du reste de la population.

Les dépôts uniquement composés de bracelets ornés sont susceptibles d'entériner cette relégation dans la durée; ils enregistreraient de la sorte les gammes ornementales promues au fil des générations. La continuité de la souveraineté, rituellement légitime jusque dans le retraitement post-mortem des parures, pourrait constituer le fil conducteur de ces dépôts, dont celui de Gross Bieberau (fig. 4, 3, 8, 10-12) (Richter 1970, p. 145). Les bracelets ornés, cochés à l'identique par paire sur leur face intérieure, sont la manifestation probable de paires non plus doubles, mais démultipliées (fig. 2, 25-26). Avec cette mise en dépôt, nous songeons, comme à Blanot, à un enfouissement unique hors sépulture d'une parure, soit une forme de relégation parmi d'autres.

Les relégations hors sépultures des prestations matrimoniales ne sont pas systématiques. Épées et bracelets ornés coexistent même parfois dans les tombes. Les inhumations doubles du Bronze final à la fois masculines et féminines d'Essfeld, de Wollmesheim et de Gammertingen (Sperber 1999, p. 605-659) pourraient indiquer dans l'optique du pouvoir utérin, non pas un chef défunt avec son épouse, mais au côté de sa génitrice ou de celle choisie pour enfanter son successeur et de ce fait porteuses des bracelets ornés: une sœur, une fille, une nièce ou toute autre épouse à l'exception de la sienne. Malgré l'emplacement imprécis des bracelets ornés, la tombe à épée de Hagenau pourrait constituer un antécédent plausible au Bronze moyen à l'instar de la tombe de Letkès en Hongrie occidentale où les bracelets se situent sur le flanc gauche à hauteur de l'épaule, séparés du corps par l'épée placée le long du bras (Pászthory et Mayer 1998, pl. 101; Kovács 1996, fig. 1a et 2).

À La Saulsotte (Aube), une sépulture triple du début du Bronze final a l'originalité d'associer un homme à deux femmes (Piette et al. 2005, p. 433-457). Un homme sans épée (A) porte aux bras deux bracelets et deux autres ornent les bras d'une adulte (C) placée tête-bêche sur sa droite (fig. 9). Les décors de ces deux paires se ressemblent, tandis que celle attribuée à la jeune femme à la gauche du défunt (B) se différencie par son ornementation. Quelle signification accorder à cette scène funéraire? Malgré une connaissance incertaine de l'ordre des inhumations, nous réitérons ici l'hypothèse monogame de deux unions matrimoniales successives (Georges 2007, p. 345). En l'absence d'études paléogénétiques, il reste que les bracelets de A et de C sont de styles nord-alpins et qu'ils fournissent un indice éventuel d'une présence étrangère (Beck 1980, pl. 7 et 53). La jeune femme porte, elle, les canons esthétiques en vigueur dans les régions de l'Yonne et de l'Aube. Si un élément de parure peut voyager sans porteur attitré, être échangé et finir sa course loin de son lieu de fabrication, son décor peut perdre aussi de 
sa signification. La chose semble exclue pour la parure de la jeune femme: son statut local se comprend dans une logique de reproduction sociale des élites, en l'occurrence celui d'une épouse, celle de l'homme de cette sépulture ou d'un tiers.

La recherche de femmes bien nées ou la quête de parures de prestige pourraient justifier des déplacements lointains. Les sémioses graphiques soulignent les tendances générales avec des territoires plus ou moins récepteurs ou producteurs en matière de graphisme.

Alfred Gell a parlé d'une cognition collective et externalisée pour expliciter l'unité de style des tatouages polynésiens (Gell 1998, p. 222). Les graphismes complexes sur bracelets de l'âge du Bronze final rhéno-alpin s'écartent de ce paradigme. Il peut être envisagé que l'affichage est de nature proto-institutionnelle avec la mise en avant de personnalités féminines aux moments cruciaux de l'ordre successoral. Dans notre hypothèse, dans les sociétés de rang, segmentées en lignées, les unions de haut rang seraient révélées par la lecture des sémioses graphiques. L'évolution, comme la variété et la répartition des graphismes viendraient éclairer d'une manière inédite l'histoire des échanges matrimoniaux. Cette proposition qui a pour elle de s'appuyer sur une grande quantité de faits archéologiques, permet également de participer au débat autour des dépôts métalliques et des multiples formes prises par la mise en terre symbolique des parures déchues.

\section{Bibliographie}

BAILLET G. 1989. Tite-Live, Histoire romaine, 5, Livre V, Paris, Les Belles Lettres.

Balac A.-M. et PAges F. 1984. Atlas préhistorique du midi méditerranéen. Feuille de Sisteron, sous la direction de G. Camps et de H. Camps-Faber, Paris, Éditions CNRS.

BARGE H. 2011. Dépôts métalliques de l'âge du Bronze. Cachettes et lieux sacrés dans les Alpes du Sud, Theix, Actilia Multimedia.

Barry L. S., Bonte P., D'OnOfrio S., Govoroff N., JAMARD J.-L., MATHieU N.-C., Porqueres i Gene E., Wilgaux J., ZeMPleni A., Zonabend F. 2000. Glossaire de la parenté, in L.S. BARRY (dir.), Question de parenté, L'Homme, n¹54-155, p. 721-732.

BECK A. 1980. Beiträge zur frühen und älteren Urnenfelderkultur im nordwestlichen Alpenvorland, Prähistorische Bronzefunde, München, C. H. Beck, XX, 2.

BOCQueT A. 1997. Archéologie et peuplement des Alpes françaises du Nord, L'anthropologie, t. 101, n 2, p. 291-393.

BOCQUET A. et coll. 1976. Les dépôts et la chronologie du Bronze final dans les Alpes du Nord, in A. BocQueT (dir.), Les âges des métaux dans les Alpes, Grenoble, colloque XXVI, U.I.S.P.P., p. 35-71.

BOUZEK J. 2011. Prehistory of Europe as seen from its centre, Czech lands from Paleolithic to the La Tène period in European context, Studia Hercynia, XV/1.

BRADLEY R. 1990. The passage of arms, an archaeological analysis of prehistoric hoards and votive deposits, Cambridge University Press, Cambridge.

BRESTRICH W. 1998. Die mittel-und spätbronzezeitlichen Grabfunde auf der Nordstadttterrasse von Singen am Hohentwiel, Kommissionverlag, Stuttgart, Konrad Theiss Verlag.

BRIQuel D. 1993. Les Étrusques, peuple de la différence, Paris, Armand Colin. 
CAmous T. 2010. Romulus, le rêve de Rome, Paris, Payot.

Cardarelli A., Pacciarelli M., Pallante P., Bellintani P. 2001. Pesi e bilance dell'età del Bronzo italiana, in C. CORTI et N. GIORDANI (dir.), Pondera, Pesi e misure nell'Antichità, Modena, p. 33-58.

CHAUME B. 1989. Les anneaux réniformes à côtes transversales du Bronze final : à propos de deux exemplaires du musée de Châtillon-sur-Seine (Côte-d'Or), Revue archéologique de l'Est, t. 40, p. 12-30.

COFfYn A. 1969. L'âge du Bronze au musée du Périgord, Gallia Préhistoire, t. XII, p. 83120.

COMBIER J. 1972. Bronze en Savoie, Albertville, Centre de documentation régionale de Tarentaise.

CORDIER G. 1984. L'âge du Bronze en Touraine, nouveaux documents, in Eléments de Préet de Protohistoire européenne, Hommages à Jacques-Pierre Millotte, Annales de l’Université de Besançon, p. 305 et suiv.

CORDIER G. 2002. Le dépôt de l'âge du Bronze final « Les Châteliers » à Amboise (Indreet-Loire) ", Revue archéologique du Centre de la France, p. 5-34.

Courtois J.-C. 1960. L'âge du Bronze dans les Hautes-Alpes, Gallia Préhistoire, 3, 1960, p. 47-108.

ČUJANOVÁ-JílKOVÁ E. 1970. Mittelbronzezeitliche Hügelgräberfelder in Westböhmen, Západoceská mohylová pohrebiste strední doby bronzové, Praha, Archeologický ústav CSAV.

De Santis A., Colacicchi O., GiUliani M. R., SANTORO B. 2010. Il processo storico nel Lazio antico tra la tarda età del bronzo e la prima età del ferro : i protagonisti, in Preistoria e Protostoria in Etruria, Atti del Nono Incontro di Studi Valentano (Vt) - Pitigliano (Gr), 12-14 Settembre 2008, L'alba dell'Etruria, Fenomeni di continuità e trasformazione nei secoli XII-VIII a.C., Ricerche e scavi, Milano, Centro Studi di Preistoria e Archeologia Onlus, p. 311-326.

DuPRÉ G. et REY P. 1969. Réflexions sur la pertinence d'une théorie de l'histoire des échanges, Cahiers internationaux de sociologie, 46, p. 133-162.

DUNNING C. et RYCHNER V. 1994. Archäologische Untersuchungen zum übergang von der Bronze - zur Eisenzeit in der Westschweiz, in Archäologische Untersuchungen zum Übergang von der Bronze - zur Eisenzeit zwischen Nordsee und Kaukasus, Regensburg, Universitätsverlag, p. 63-97.

EBERSCHWEILER B. 1998. Ein rätselhafter Holstab aus dem Zürichsee, Archéologie suisse, t. $21, \mathrm{n}^{\circ} 3$, p. $98-102$.

ELUÈRE C. 1974. Anneaux de jambes et jambières à spirales de France, Bulletin de la Société préhistorique française, t. 71, fasc. 2, p. 543-566.

EluÈRE C. 1975. Les anneaux réniformes de France, Bulletin de la Société préhistorique française, t. 72, fasc. 1, p. 26-32.

FATH B. 2010. Textilien und Symbole für ihre Herstellung in eisenzeitlichen Gräbern Mitteleuropas Griechenland - Este - Frög - Sopron, Praehistorische Zeitschrift, t. 86, p. 254271. 
FISCHER V. 2012. Les bronzes en contexte palafittique sur les rives du Léman et des Trois-Lacs (Suisse occidentale), Cahiers d'Archéologie romande, Lausanne, n¹28.

Fontanille J. et Beyaert-Geslin A. 2006. Sémiologie et sémiotique, in S. Mesure et P. SAVIDAN (dir.), Dictionnaire des Sciences humaines, Paris, PUF, p. 1071 et suiv.

FRAZER J. G. 1981. Le Rameau d'Or, Le Roi magicien dans la cité primitive, chap. XVIII [1 $1^{\text {ère }}$ ed. 1922], Paris, Laffont.

GAMBARI F. M. 1997. La prima età del Ferro nel Piemonte nord-occidentale, in La valle d'Aosta nel quadro della Preistoria e Protostoria dell'arco alpino centro-occidentale, Atti della XXXI riunione scientifica dell'IIPP, Courmayeur 2-5 juin 1994, Florence, IIPP, p. 341-360.

GELL A. 1998. Art and agency, an anthropological theory, Oxford, Clarendon Press.

GEORGES V. 2007. Le Forez $d u v^{e}$ au $I^{e r}$ millénaire avant J.-C. Territoires, identités et stratégies des sociétés humaines du Massif Central dans le bassin amont de la Loire (France), Thèse de doctorat en Archéologie, Dijon, Université de Bourgogne, 2007, 2 vol. [http:/ / tel.archives-ouvertes.fr ; tel-00369820, version 1].

GEORGES V. à paraître. L'ornementation des bracelets à l'âge du Bronze européen : analyse descriptive et perspectives, in C. MORDANT C. et S. WIRTH (dir.), Normes et variabilités au sein de la culture matérielle des sociétés de l'âge du Bronze, Journée thématique 13 juin 2013, Société préhistorique française et Association pour la Promotion des Recherches sur l’Âge du Bronze.

Gimbutas M. 1965. Bronze Age cultures in Central and Eastern Europe, de Gruyter, Mouton and Co publishers.

GLUCHY A. 2013. Les techniques du bronzier à l'âge du Bronze : méthodologie d'une lecture des états de surface d'objets de bronze à partir de l'étude du décor de bracelets du dépôt $n^{\circ} 2$ de SaintPriest Les Feuilly (Rhône), Thèse de doctorat en Archéologie, Dijon, Université de Bourgogne.

GOMEZ de SOTO J. 1993. Pictogrammes, figurations anthropomorphes et zoomorphes sur les céramiques de la fin de l'Âge du Bronze, une révision, in La représentation humaine du Néolithique à l'âge du Fer, Actes du $113^{\mathrm{e}}$ Congrès national des Sociétés historiques et scientifiques, Avignon 1990, Paris, Éditions du CTHS, p. 149-162.

GRANET M. 1939. Catégories matrimoniales et relations de proximité dans la Chine ancienne, Paris, Alcan.

KAUL F. 1998. Ships on bronzes. À study in Bronze Age religion and iconography, Copenhague, Publications from the National Museum Studies in Archaeology and History, 3 vol., fasc. 1-2.

KÉROUANTON I. 2002. Le lac du Bourget (Savoie) à l'âge du Bronze final : les groupes culturels et la question du groupe du Bourget, Bulletin de la Société préhistorique française, t. $99, \mathrm{n}^{\circ} 3$, p. $521-561$.

KOVÁCS T. 1996. The Tumulus Culture in the Middle Danube Region and the Carpatian Basin : Burials of the Warrior Elite, in C. BELARDELLI et R. PERONI, The Bronze Age in Europe and the Mediterranean, Colloquium XXII, The Evolution of Settlement Systems and Society in Europe and the Mediterranean during the Bronze Age and its Pre- and Protourban Developments, XIII international Congress of prehistoric and protohistoric Sciences, Forli (Italia), 8-14 September 1996, Forli, A.B.A.C.O. Edizioni, p. 201-207. 
KUBACH W. 1991. Das Rhein-Main-Gebiet zwischen Hügelgräber - und Urnenfelderkultur, in M. GEDL (dir.), Die Anfänge der Urnenfelderkulturen in Europa, Począ tki kultur pól popielnicowych w Europie, Archaeologia Interregionalis, 13, Warszawa, p. 141-156.

LAGRAND C. 1968. Recherches sur le Bronze final en Provence méridionale, thèse de l’Université d'Aix-en-Provence.

LAGRAND C. 1976. Les civilisations de l'âge du Bronze en Provence, Le Bronze final, in J. GuILAINE (dir.), La Préhistoire française, Paris, CNRS, t. II, p. 452-458.

Laux F. 1971. Die Bronzezeit in der Lünerburg Heide, Hildesheim, August Lax Verlagsbuchhandlung.

LEDUC C. 1991. Comment la donner en mariage ? La mariée en pays grec (IX ${ }^{\mathrm{e}}-\mathrm{IV}^{\mathrm{e}}$ siècle avant J.-C.), in P. SCHMitT PANTEL (dir.), Histoire des femmes en Occident, L'Antiquité, t. 1, Plon, Paris, p. 259-316.

LeroI-GOUhran A. 1964. Le geste et la parole, I, Technique et langage, Paris, Albin Michel.

LÉvi-Strauss C. 1984. Paroles données, Paris, Plon, 1984.

LÉVI-StRAuss C. 2008, La voie des masques, in DebAENE V. et al., OEuvres, Bibliothèque de la Pléiade, p. 873-1050.

MeILlassoux C. 2008. Femmes, greniers et capitaux, $2^{\mathrm{e}}$ édition, Paris, L'Harmattan.

MERCURIN R. en collaboration avec CAMPOLO S. 2011. Les dépôts d'objets en bronze dans les Alpes-Maritimes à l'âge du Bronze final : état des lieux et perspectives de recherche, in D. GARCIA (dir.), L'âge du Bronze en Méditerranée, Recherches récentes, Paris, Errance, p. 131154.

MiLCENT P.-Y. 2004. Le premier âge du Fer en France centrale, Mémoire de la Société préhistorique française, 34, Paris, 2 vol.

Millotte J.-P. 1963. Le Jura et les plaines de Sâ̂ne aux âges des métaux, Annales littéraires de l’Université de Besançon, vol. 59.

Millotte J.-P. 1993. À la recherche des artisans et commerçants de l'âge du Bronze, L'exemple du dépôt de Larnaud (Jura) : essai sur les apports de la typologie, in Mélanges offerts à J.-J. HATT, Cahiers alsaciens d'Archéologie d'Art et d'Histoire, t. XXXVI, Strasbourg, p. 35-52.

Millotte J.-P. et VignaRd M. 1960. Catalogue des collections archéologiques de Lons-leSaunier, Les antiquités de l'âge du Bronze, Annales littéraires de l'Université de Besançon, vol. 22.

MOHEN J.-P. 1977. L'âge du Bronze dans la région de Paris, Catalogue synthétique des collections conservées au Musée des Antiquités nationales, Paris, Éditions des Musées nationaux.

MOOSLEITNER F. 1991. Bronzezeit im Saalfeldener Becken, Archäologie in Salzburg, Band 1.

MÜlleR-KARPE H. 1949-50. Grünwalder Gräber, Praehistorische Zeitschrift, 34-35, p. 313325.

MÜLlER-KARPE H. 1959. Beiträge zur Chronologie der Urnenfelderzeit nördlich und südlich der Alpen, Römisch-Germanische Forschungen, Bd. 22, Berlin, Walter de Gruyter. 
MülleR-KARPE H. 1980. Handbuch der Vorgeschichte, Band IV, Bronzezeit, Drietter Teilband Tafeln, Münschen, C. H. Beck'sche Verlagbuchandlung.

O' CONNOR B. 1980. Cross-Channel relations in the Later Bronze Age, Oxford, British Archeological Reports.

OBERKAMPF M. et coll., 1997. Âge du Bronze de Haute-Savoie. t. 1 : en dehors des stations littorales, Annecy, Musée-Château d'Annecy.

PALTINERI S. et RUBAT BOREL F. 2010. Le armille tipo Zerba : un riesame della questione, in Preistoria e Protostoria in Etruria, Atti del Nono Incontro di Studi Valentano (Vt) - Pitigliano (Gr), 12-14 Sett. 2008, L'alba dell'Etruria, Fenomeni di continuità e trasformazione nei secoli XIIVIII a.C., Ricerche e scavi, Centro Studi di Preistoria e Archeologia - Onlus, p. 609-622.

PÁSZTHORY K. 1985. Der bronzezeitliche Arm- und Beinschmuck in der Schweiz, Prähistorische Bronzefunde, X, 3, München, C. H. Beck.

PÁSzTHORY K. et MAYER E. F. 1998. Die Äxte und Beile in Bayern, Prähistorische Bronzefunde, IX, 20, München, C. H. Beck.

PietTe J., ROtTIER S., DEPIERRE G. 2005. Les pratiques funéraires au début du Bronze final dans les nécropoles de Barbuise-Courtavant et de La Saulsotte (Aube), in C. MORDANT et G. DEPIERRE (dir.), Les pratiques funéraires à l'âge du Bronze en France, CTHS et Société archéologique de Sens, Paris et Sens, p. 433-457.

PININGRE J.-F. 2014. À propos du dépôt de Mathay (Doubs, France) : quelques réflexions sur les dépôts de parures féminines de prestige au Bronze final, in R.M. ARBOGAST et A. GREFFIER-RICHARD (dir.) Entre archéologie et écologie, une Préhistoire de tous les milieux, Mélanges offerts à Pierre Pétrequin, Besançon, Presses universitaires de Franche-Comté, Annales Littéraires de l'Université de Franche-Comté, 928 ; série "Environnement, sociétés et archéologie », 18, p. 399-416.

REIM H. 1994. Archäologische Untersuchungen zum Übergang von der Bronze- zur Eisenzeit in Baden-Wütermberg, in Archäologische Untersuchungen zum Übergang von der Bronze - zur Eisenzeit zwischen Nordsee und Kaukasus, Ergebnisse eines Kolloquiums in Regensburg, Bonn, Dr. Rudolf Habelt, p. 99-125.

RENOU L. 2004. Hymnes spéculatifs du Véda, traduit du sanskrit et annoté, 1 ère éd. 1956, Paris, Gallimard.

RICHTER I. 1970. Der Arm - und Beinsmuck der Bronze - und Urnenfeldderzeit in Hessen und Rheinhessen, Prähistorische Bronzefunde, X, 1, München, C. H. Beck.

Rolley C. (dir.) 2003. La tombe princière de Vix, Paris, Picard, 2 vol.

ROWLANDS M. J. 1998. Kinship, alliance and exchange in the European Bronze Age, in K. KRISTIANSEN et M. ROWLANDS (dir.), Social transformations in Archaeology, global and local perspectives, London, New York, Routledge, p. 142-182.

RubAt Borel F. 2009. Tipologia e cronologia degli elementi del ripostiglio di Chiusa di Pesio, in M. V. GAMBARI (dir.), Il Ripostiglio del Monte Cavanero di Chiusa di Pesio (Cuneo), Allessendria, LineLab. edizioni, p. 55-105.

RYCHNER V. 1979. L'âge du Bronze final à Auvernier (Lac de Neuchatel, Suisse), Typologie et chronologie des anciennes collections conservées en Suisse, Cahiers d'Archéologie romande, Bibliothèque vaudoise, $\mathrm{n}^{\circ} 15$ et 16 , Auvernier 1 et 2 . 
RYCHNER-FARAGGI A.-M. 1993. Hauterive-Champréveyres 9, Métal et parure au Bronze final, Neuchâtel, archéologie neuchâteloise, 17.

RYCHNER V., BÖHRINGER S., GASSMANN P. 1996. Dendrochronologie et typologie du Bronze final dans la région de Neuchâtel (Suisse) : un résumé, in Absolute chronology archaeological Europe 2500-500 BC., Acta Archaeologica, vol. 67, Kopenhagen, p. 307-314.

SCHAEFFER F. A. 1926. Les tertres funéraires préhistoriques dans la Forêt de Haguenau, t. II, Les tumulus de l'âge du Fer, Musée de Haguenau.

SCHEID J. 2005. Quand faire, c'est croire. Les rites sacrificiels des Romains, Paris, Aubier, Collection historique.

SCHUBART H. 1972. Die Funde der älteren Bronzezeit in Mecklenburg, Neumünster, Karl Wachholtz Verlag.

SCHUMACHER-MATTHAÜS G. 1985. Studien zu bronzezeitlichen Schmucktrachten im Karpatenbecken. Ein Beitrag zur Deutung der Hortfunde im Karpatenbecken, Mainz am Rhein, Philipp von Zabern.

SIEPEN M. 2005. Der hallstattzeitliche Arm- und Beinschmuck in Österreich, Prähistorische Bronzefunde, X, 6, Stuttgart, Steiner.

SimON-MiLlot R. 1998. Les bracelets en bronze du dépôt de Larnaud (Jura) conservés au Musée des Antiquités nationales, Antiquités nationales, nº 30, p. 25-86.

SPERBER L. 1999. Zu den Schwertträgern im westlichen Kreis der Urnenfelderkultur: Profane und Religiöse Aspekte, in Eliten in der Bronzezeit: Ergbnisse zweier Kolloqien in Mainz und Athen, Mainz, Verlag des Römisch-Germanischen Zentralmuseums, Monographien des RGZM, 43.2, p. 605-659.

SPROCKHOFF E. 1956. Jungbronzezeitliche Hortfunde, Der Südzone des Nordischen Kreises (Periode V), Romisch-Germanisches Zentralmuseum zu Mainz, Katalog 16, Mainz, Verlag RGZM.

STUCHLIK S. 1992. Die Veterov-Gruppe und die Entstehung der Hügelgräberkultur in Mähren, Praehistorische Zeitschrift, t. 67, n 1, p. 15-42.

Thevenot J.-P. et coll. 1991. L'âge du Bronze en Bourgogne. Le dépôt de Blanot (Côte-d'Or), Supplément à la Revue archéologique de 1’Est, n¹1, Dijon.

TIZZONI M. 1976. Il ripostiglio del Bronzo finale di Zerba (Piacenza), in Atti della XIX riunione scientifica IIPP XIX in Emilia Romagna, Firenze, p. 311-326.

TODD E. 2011. L'origine des systèmes familiaux, t. 1, L'Eurasie, Paris, Gallimard.

UENZE O. 1949-50. Die Hortfunde von Allendorf, Praehistorische Zeitschrift, t. 34-35, p. 202220.

VÉBER C. 2009. Métallurgie des dépôts de bronzes à la fin de l'âge du Bronze Final (IXe-VIII av. J. C.) dans le domaine Sarre-Lorraine: essai de caractérisation d'une production bronzière au travers des études techniques : formage et analyses élémentaires, British archeological Reports, International Séries, Oxford, Archaeopress.

VERGER S. 1992. L'épée du guerrier et le stock de métal : de la fin du Bronze ancien à l'âge du Fer, in G. KAENEL et Ph. CURDY (dir.), L'âge du Fer dans le Jura, Actes du Quinzième colloque de l'AFEAF, 9-12 mai 1991, Pontarlier et Yverdon-les-Bains, Cahiers d'Archéologie romande, 57, Lausanne, p. 135-151. 
Verger S. 1998. Les trois âges de la Dame de Blanot, in C. Mordant, M. Pernot et V. RYCHNER (dir.), L'atelier du bronzier en Europe, $d u X X^{e}$ au VIII ${ }^{e}$ siècle avant notre ère, Actes du colloque international Bronze 96, Neuchâtel et Dijon 1996, t. 3, Production, circulation et consommation $d u$ bronze, Comité des Travaux historiques et scientifiques et CRTGR, Paris, Dijon, p. 33-39.

VERGER S. 2010. Résidences aristocratiques et espaces publics de l'Italie du Nord et de l'Allemagne du sud, Dossiers de l'Archéologie, 339, Archeologia, Dijon, p. 66-73.

VITAL J. 1988. Le groupe Rhin Suisse France orientale dans la moyenne vallée du Rhône, in P. BRUN et C. MORDANT (Dir.) Le groupe Rhin-Suisse-France orientale et la notion de civilisation des Champs d'Urnes, Actes du colloque international de Nemours 1986, Mémoires du Musée de Préhistoire d’Ile-de-France n¹, Nemours, A.P.R.A.I.F., p. 445457.

Wels-Weyrauch U. 1991. Die Anhänger in Südbayern, Prähistorische Bronzefunde, IX, 5, München, C. H. Beck.

WiLhelmi K. 1983. Die Jüngere Bronzezeit zwischen Niederrhein und Mittelweser, Kleine Schriften aus dem vorgeschichtlichen seminar Marburg.

WIRTH S. 2006. Le mystère de la barque solaire : quelques considérations à propos des décors sur les situles de type Hajdùböszörmeny, in L. BARAY (dir.), Artisanats, sociétés et civilisations, Hommage à Jean-Paul THEVENOT, Supplément à la Revue archéologique de l'Est, 24, Dijon, p. 331-345.

Zumstein H. 1965. L'âge du Bronze dans le département du Haut-Rhin, Revue archéologique de l'Est, t. XV, fasc. 1-4, t. XVI, fasc. 1-3. 


\section{Illustrations}

Figure 1: Variété des ornementations complexes de l'aire rhénane et des Alpes occidentales ; 1. Gross Bieberau (Richter 1970, pl. 48, n878) ; 2. Kelsterbach (ibid., pl. 17, n³22) ; 3. Mörigen (Pászthory 1985, pl. 120, n¹348) ; 4. Grandson-Corcelettes (ibid., pl. 100, $\left.\mathrm{n}^{\circ} 1215\right)$; 5. Morges (ibid., pl. 59, n773) ; 6. Villa Nessi (Tizzoni 1976, fig. 3E) ; 7-8. L'Épine (Courtois 1960, fig. 49, $\mathrm{n}^{\circ} 1$ et fig. 50, n³) ; 9. Digne (Barge 2011, p. 74 et Musée d'archéologie nationale) ; 10. Cimiez (Mercurin et Campolo 2011, fig. 8, n²).
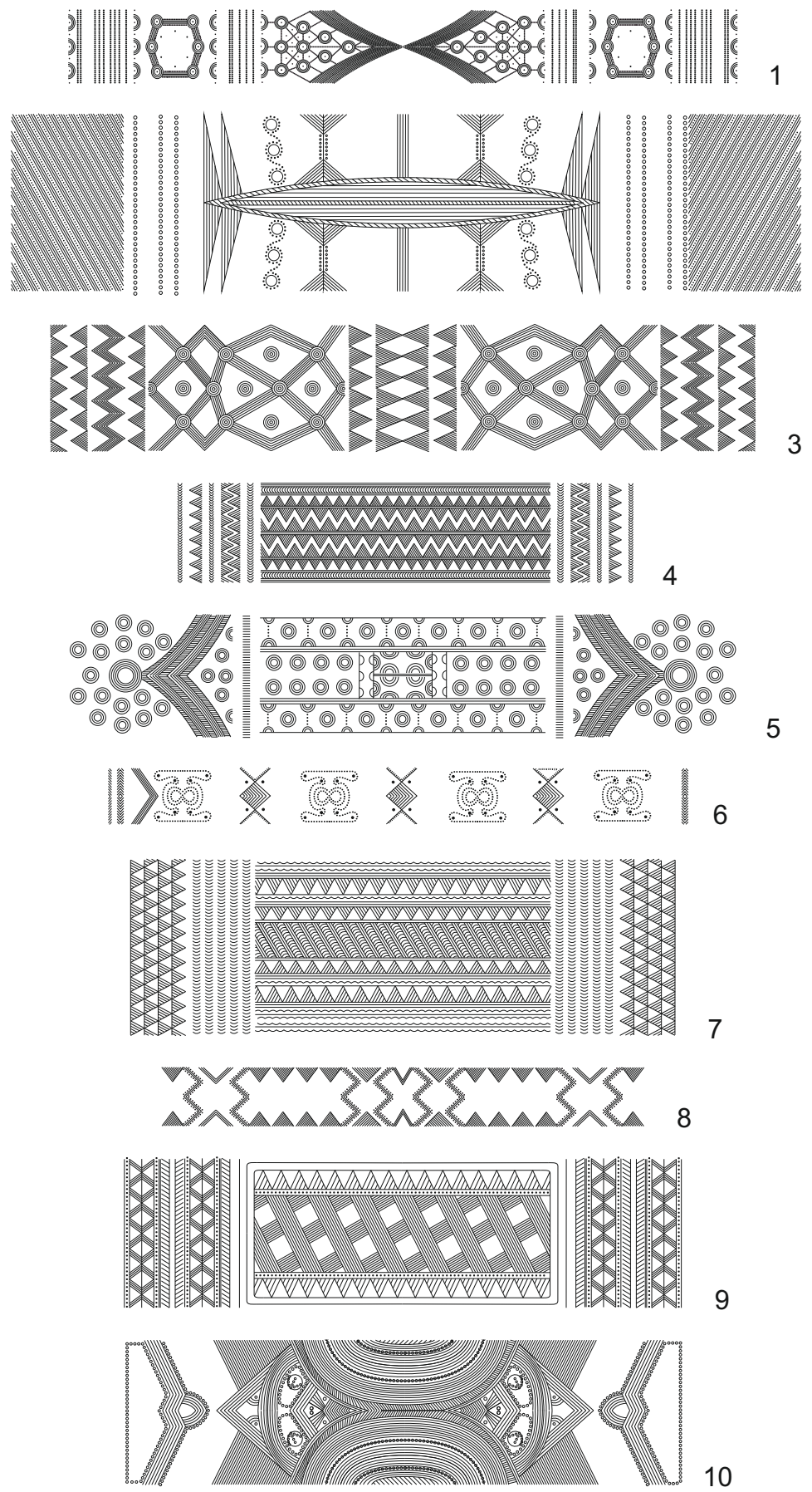

0 $10 \mathrm{~cm}$ 
Figure 2: Déroulés linéaires orthogonaux des occurrences de l'arbre stylistique élargi et simplifié des bracelets des types Cortaillod et Corcelettes, XVI-IX siècle av. J.-C. (en grisé : partie illisible ou non conservée) ; 1 . Hohentwiel (Brestrich 1998, pl. 1, nº4); 2. Vinols (Georges 2007, fig. 123, D3) ; 3. Bevaix (Pászthory 1985, pl. 67, nº32) ; 4. Provenance indéterminée (ibid., pl. 67, n830) ; 5. Provenance indéterminée (Coffyn 1969, fig. 11, n²4); 6. Albertville (Bocquet et coll. 1976, fig. 5, n4) ; 7. Corsier (Pászthory 1985, pl. 67, n834) ; 8. Menthon-Saint-Bernard (Oberkampf et coll. 1997, pl. 38, n`3); 9. Pagny-la-Ville (Millotte 1963 : pl. 40, n³) ; 10 Baume-les-Messieurs (Millotte et Vignard 1960, pl. XVIII, $\mathrm{n}^{\circ} 161$ ) ; 11-12. "Orpund » (Pászthory 1985, pl. 67, n833 [12] et 835 [11]) ; 13-15. SaintPriest (Gluchy 2013, fig. 78, n`512 et fig. 179, n`3; ibid., fig. 77, $\mathrm{n}^{\circ} 608$; ibid., fig. $179, \mathrm{n}^{\circ} 4$ [n $\left.{ }^{\circ} 14\right]$; 16-17. Amboise (Cordier 2002, fig. 6, n० 14 et 7) ; 18. "de Rives à Concise » (Oberkampf et coll. 1997, Pl. 54, n`3) ; 19. Auvernier (Rychner 1979, pl. 91, n6) ; 20. Cortaillod (Pászthory 1985, pl. 73, n888) ; 21. Larnaud (Simon-Millot 1998, pl. 18, I) ; 22. Bevaix (Pászthory 1985, pl. 73, n890) ; 23. Zürich-Wollishofen (ibid., pl. 73, n885); 24. Guévaux (ibid., pl. 157, n¹763) ; 25-26. Sursee (ibid., pl. 75, n897 et 898) ; 27-28. Estavayer (ibid., pl 70, n॰854 et 855 et pl. 69, n॰852) ; 29. Avenches (ibid., pl. 68, n॰842) ; 30. Estavayer

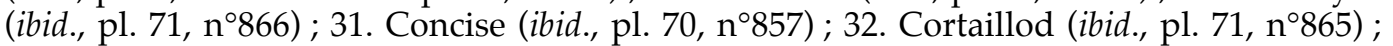
33. Aux environs de Loèche-les-Bains (ibid., pl. 71, n867-868); 34. Provenance indéterminée (ibid., pl. 72, n872) ; 35. Avenches, Murtensee (ibid., pl. 120, n¹344A) ; 36. Cortaillod (ibid., pl. 120, n ${ }^{\circ} 1344$ ) ; 37. Auvernier (ibid., pl. 120, n` 1346) ; 38. Estavayer (ibid., pl. 120, ${ }^{\circ} 1345$ ) ; 39. Corcelettes (ibid., pl. 119, n ${ }^{\circ} 342$ ); 40. Auvernier (ibid., pl. 126, $\left.\mathrm{n}^{\circ} 1380\right)$; 41. Concise (ibid., pl. 124, $\left.\mathrm{n}^{\circ} 1368\right)$; 42. Auvernier (Rychner 1979, pl. 85, n6) ; 43. Mörigen (Pászthory 1985, pl. 121, n $\left.{ }^{\circ} 1350\right) ; 44$. Cortaillod (ibid., pl. 124, $\mathrm{n}^{\circ} 1371$ ) ; 45. Mörigen (ibid., pl. 120, $\mathrm{n}^{\circ} 1348$ ) ; 46. Auvernier (ibid., pl. 129, $\mathrm{n}^{\circ} 1402$ ) ; 47. Cortaillod (ibid., pl. 128, n 1397) ; 48. Bâle (ibid., pl. 119, $\left.{ }^{\circ} 1338\right)$; 49. Saint-Prex (ibid., pl. 129, n¹403) ; 50. Hochstadt (Richter 1970, pl. 60, n¹053) ; 51. Corcelettes (Pászthory 1985, pl. 103, n 1238 et 1239) ; 52. Auvernier (ibid., pl. 102, n॰1233) ; 53. Corcelettes (ibid., pl. 104, n॰ ${ }^{\circ} 1243$ ) ; 54. idem (ibid., pl. 113, n¹299) ; 55. idem (ibid., pl. 112, n¹294) ; 56. idem (ibid., pl. 106, n 1250 et 1251) ; 57. Mörigen (ibid., pl. 109, $\mathrm{n}^{\circ} 1276$ ). 

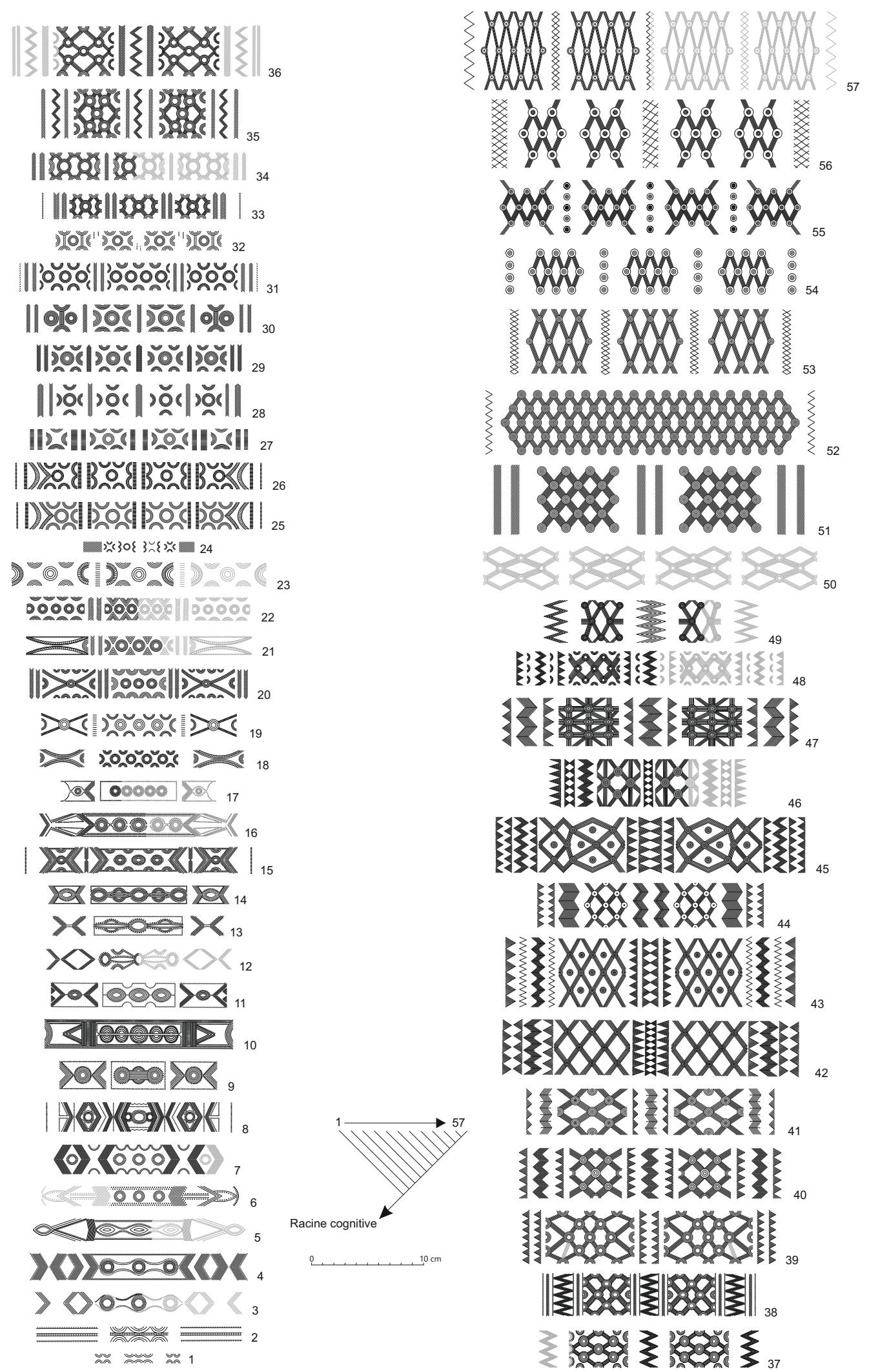

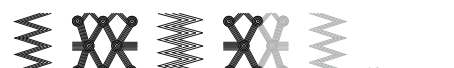

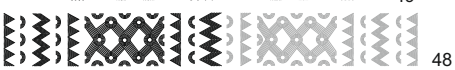

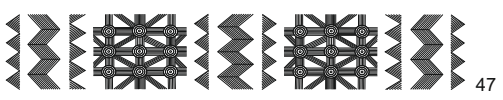

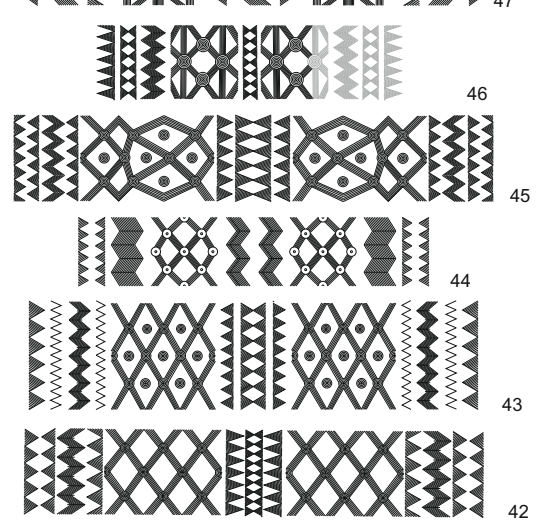

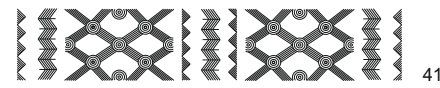

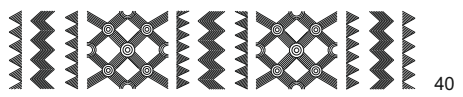

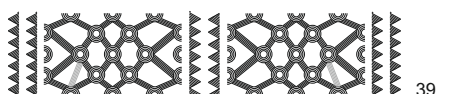

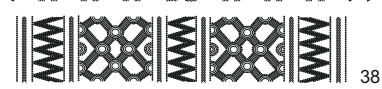

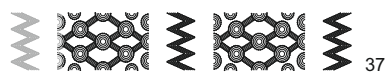


Figure 3 : Déroulés linéaires orthogonaux des occurrences de l'arbre stylistique élargi du type Auvernier avec hachures obliques centrales, XIV-IX siècle av. J.-C. (1-10) avec hachures obliques et bracelets longilignes du début du Bronze final à décors analogues (en grisé : partie illisible ou non conservée) ; 1 . Zimmersheim (Zumstein 1965, n 450); 2. Moosseedorf (Beck 1980, pl. 1, C, 4) ; 3. Brison-Saint-Innocent (Kérouanton 2002, fig. 28, $\left.\mathrm{n}^{\circ} 14\right) ; 4-5$. Auvernier (Pászthory 1985, pl. 98, n¹197 et Rychner 1979, pl. 90, n¹0); 6. Hauterive-Champréveyres (Rychner-Faraggi 1993, pl. 77, n5) ; 7. Auvernier (Pászthory 1985, pl. 97, $\mathrm{n}^{\circ} 1196$ ) ; 8. Vallamand (ibid., pl. 98, n ${ }^{\circ} 200$ ) ; 9. Chabrey (ibid., pl. 100, $\left.\mathrm{n}^{\circ} 1216\right)$; 10. L’Épine (Courtois 1960, fig. 49, n`1 et fig. 50, n³) ; 11. Spiez (Beck 1980, pl. 2, A, 4) ; 12. Clans (Lagrand 1976, fig. 2, n²0) ; 13. Montverdun (Georges 2007, pl. 161, nº ) ; 14. Hauterive-Champréveyres (Rychner-Faraggi 1993, pl. 77, n ${ }^{\circ}$ ); 15. Pérouges (Vital 1988, fig. 2, n5) ; 16. Aislingen (Wels-Weyrauch 1991, pl. 48, B, nº 23 et 24). 

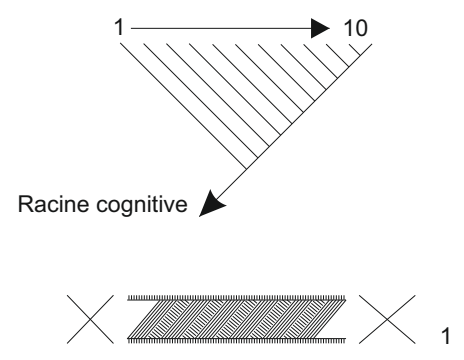

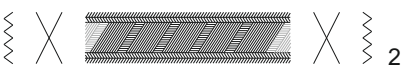

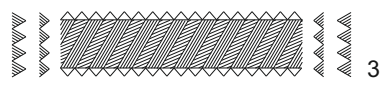
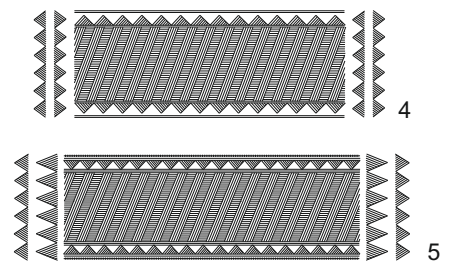

84

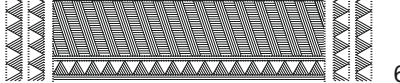

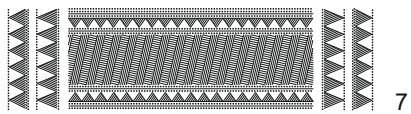

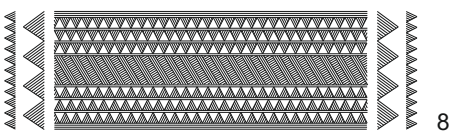

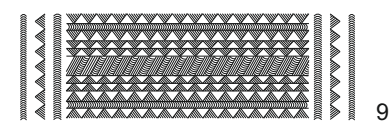

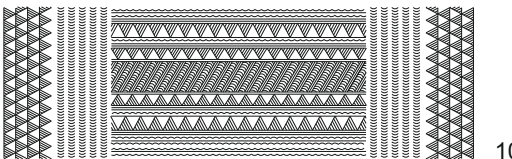

Din

KII

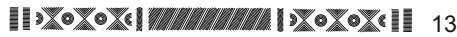

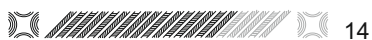

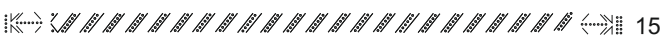

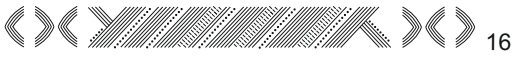
$10 \mathrm{~cm}$ 
Figure 4 : Déroulés linéaires orthogonaux des occurrences de l'arbre stylistique incluant des bracelets dits réniformes, XVI-IX ${ }^{\mathrm{e}}$ siècle av. J.-C. (en grisé : partie illisible ou non conservée) ; 1. Hagenau (Pászthory et Mayer 1998 : pl. $101:$ n $\left.^{\circ} 25\right) ; 2$. Štáhlavy (ČujanováJílková 1970, pl. 114, n¹20); 3. Unterbrunnham (Müller-Karpe 1949, fig. 16, n²) ; 4. Praha-Bubeneč 2 (Kytlicova 2007, pl. 42, B, n²) ; 5. Zelené (Čujanová-Jílková 1970, pl. 93, $\mathrm{n}^{\circ} 1$ ) ; 6. Borotice (Stuchlik 1992, fig. 24, $\mathrm{n}^{\circ} 2$ et 3) ; 7. Praha-Bubeneč 2 (Kytlicova 2007, fig. 42, B, n³) ; 7bis. Mathay (Piningre 2014 et Musée de Montbéliard) ; 8. Larnaud (SimonMillot 1998, pl. 16, C) ; 9. Gammertingen (Müller-Karpe 1980, pl. 437, C, n²) ; 10. Worms (Richter 1970, pl. 47, n॰866 et 867); 11. Gross Bieberau (ibid., pl. 47, n871 et 872); 12. Dienheim (ibid., pl. 47, n०869); 13. Wolfsheim (ibid., pl. 47, n'864); 14. Geisenheim (ibid., pl. 47, n874) ; 15. Wisselsheim (ibid., pl. 47, n875) ; 16. Gross Bieberau (ibid., pl. 47, $\mathrm{n}^{\circ} 876$ ) ; 17. Pfeddersheim (ibid., pl. 48, n॰877) ; 18-21. Gross Bieberau (ibid., pl. 48, n879, 878, 881 et 873) ; 22. Sinsheim (Lindenschmit 1900, pl. 47, $\mathrm{n}^{\circ} 1$ ) ; 23. Provenance indéterminée (Richter 1970, pl. 49, n890) ; 24. Kleedorf (Müller-Karpe 1959, pl. 140, A, $\mathrm{n}^{\circ} 6$ ) ; 25. Blanot (Thevenot et coll. 1991, fig. 37) ; 26. Boutigny (Mohen 1977, n³33) ; 27-28. [?] (Richter 1970, pl. 48, n 880 et 884) ; 29-30. Lindenstruth (ibid., pl. 49, n 889 et 888) ; 31. Bekdorf (Laux 1971, pl. 56, n5) ; 32. Champigny-sur-Aube (Eluère 1974, fig. 10, n²) ; 33. Courdemanges (Eluère 1975, fig. 1, n5) ; 34. Lauingen (Sprockhoff 1956 : pl. 44, n³) ; 35. Bingen (Richter 1970, pl. 49, n' 887 ) ; 36. Châtillon-sur-Seine (Chaume 1989, fig. 1, $\mathrm{n}^{\circ} 5$ ) ; 37. Provenance indéterminée (Chaume 1989, fig. 4) ; 38. Lauingen (Sprockhoff 1956 : pl. 44, ${ }^{\circ} 1$ ) ; 39-40. Münster Handorf (Wilhelmi 1983 : fig. 31, en bas et en haut) ; 41. Vadrup (Sprockhoff 1956, pl. 44, n5); 42. Aoste (Chaume 1989, fig. 2, nD2); 43. Saalfelden (Moosleitner 1991, pl. 20, n²6). 


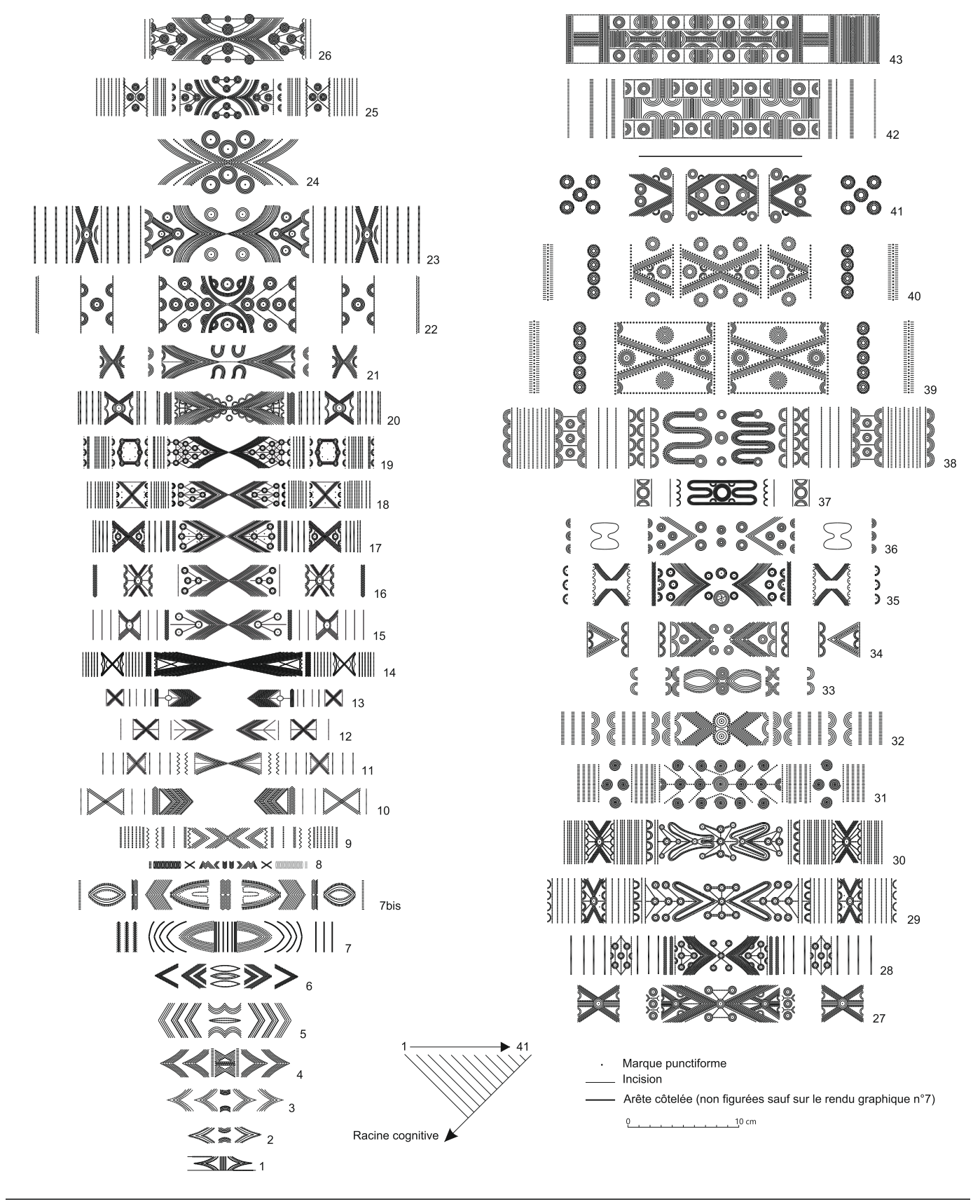


Figure 5A: Espaces de communication mettant en jeu des sémioses graphiques particulières au cours de la phase évoluée du Bronze final. 1. Répartition des branches 3957 de la fig. 2 ; 2 . Branches $1-10$ de la fig. 3 ; 3. Branches $9-41$ de la fig. 4 ; 4 et 5 . Branches des fig. 5B et C ; 6. Dépôt de Larnaud ; 7. Bracelets réniformes d'Aoste et Saalfelden.

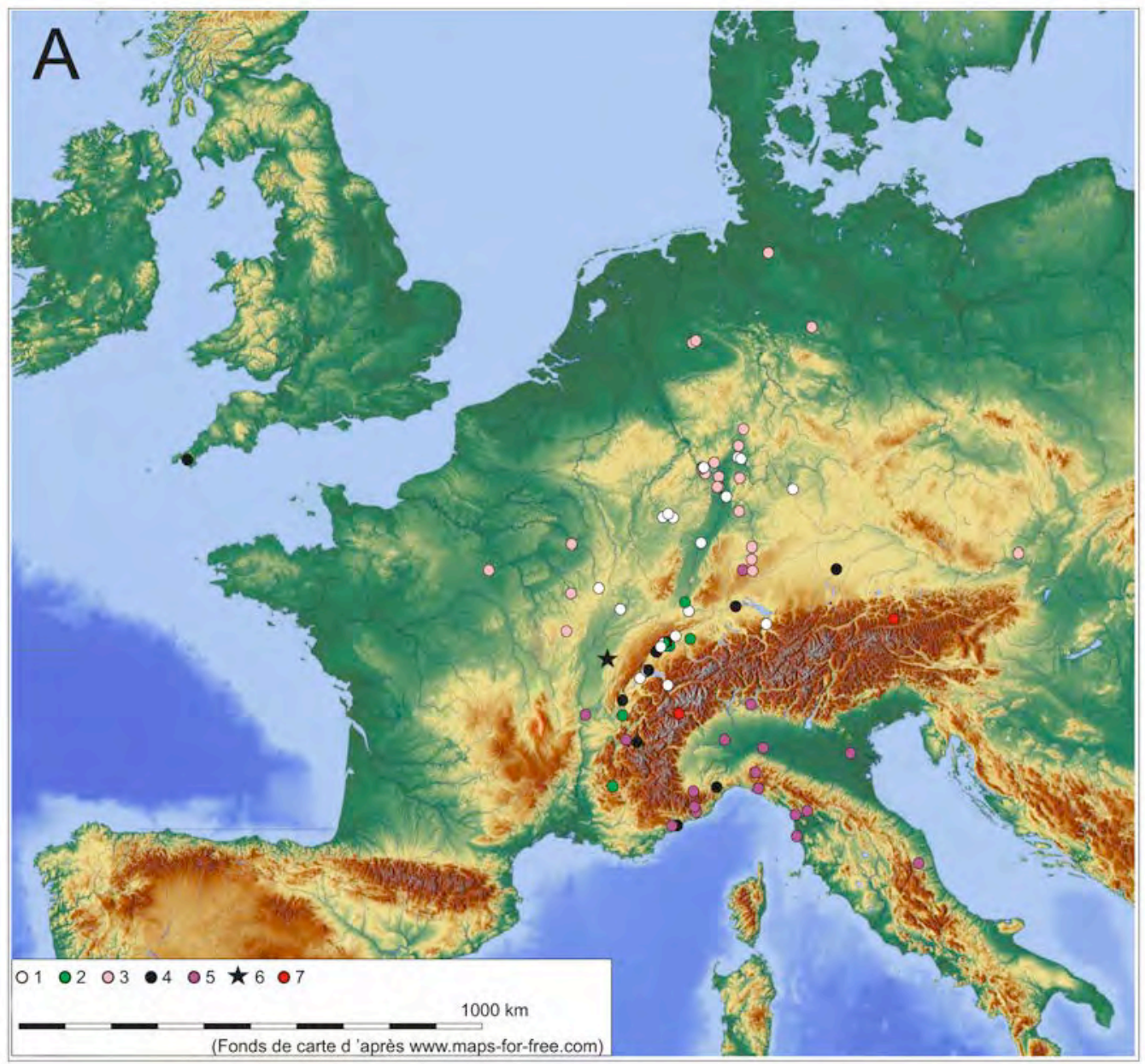

Figure 5B: Déroulés linéaires orthogonaux des occurrences de l'arbre stylistique du «style Helston », XII- $X^{\mathrm{e}}$ siècle av. J.-C. (en grisé : partie illisible ou non conservée) ; 1. Grandson-Corcelettes (Pászthory 1985, pl. 49, n656) ; 2-3. Auvernier (ibid., pl. 48, n642 et 643) ; 4. Feldgeding (Müller-Karpe 1959, pl. 190, K, 2) ; 5. Seyssel (Oberkampf et coll. 1997, pl. 51, n॰7) ; 6-7. Morges (Pászthory 1985, pl. 55, n॰737 et pl. 56, n749) ; 8. Saint-Sorlin (Combier 1972, pl. 31); 9-10. Larnaud (Simon-Millot 1998, pl. 17, Q et S) ; 11. Unterstammheim (Beck 1980, pl. 1, B, 4) ; 12. Helston (O' Connor 1980, fig. 16, C, 1) ; 1314. Larnaud (Simon-Millot 1998, pl. 17, P et L) ; 15-16. Morges (Pászthory 1985, pl. 158, $\mathrm{n}^{\circ} 1774$ et 1775) ; 17. Cimiez (Mercurin et coll. 2011, fig. 8, $\mathrm{n}^{\circ} 2$ ) ; 18. Cairo Montenotte (Gambari 1997, fig. 4).

Figure 5C : Déroulés linéaires orthogonaux des occurrences de l'arbre stylistique du type Zerba, XII-IX ${ }^{\mathrm{e}}$ siècle av. J.-C. (en grisé : partie illisible ou non conservée) ; 1-2. Borniga di Realdo (Paltineri et Rubat Borel 2010, fig. 1, n50 et 51) ; 3. Saint-Priest (Gluchy 2013, fig. 79, $\mathrm{n}^{\circ} 375$ ) ; 4-5. Chiusa di Pesio (Rubat Borel 2009, fig. 47, $\mathrm{n}^{\circ} 1$ et 2) ; 6-7. Larnaud (SimonMillot 1998, pl. 12, C et B) ; 8. Goncelin (Bocquet et coll. 1976, fig. 4, $\mathrm{n}^{\circ} 7$ ) ; 9. Pezzana (Tizzoni 1976, fig. 4, B) ; 10. Loto (ibid., fig. 6 en bas) ; 11. Pfeffingen (Müller-Karpe 1959, 
pl. 165, A, 8) ; 12. Limone di Livourno (Tizzoni 1976, fig. 8, A, 2) ; 13. Badia Paveze (ibid., fig. 4 au milieu et fig. 5) ; 14. Amboise (Cordier 1984, fig. 1, n5) ; 15. Zerba (Tizzoni 1976, fig. 1 et 2) ; 16-17. Pariana (Paltineri et Rubat Borel 2010, fig. 1, n`35 et 36) ; 18-19. Larnaud (Simon-Millot 1998, pl. 8, H et pl. 18, E) ; 20. Monte-Bignone (Paltineri et Rubat Borel 2010, fig. 2, $\mathrm{n}^{\circ} 31$ ) ; 21-22. Cimiez (Mercurin et coll. 2011, fig. 7, $\mathrm{n}^{\circ} 1$ et fig. $6, \mathrm{n}^{\circ} 1$ ) ; 23. Villa Nessi (Tizzoni 1976, fig. 3, E) ; 24. Limone di Livourno (Tizzoni 1976, fig. 8, A, 1) ; 25. Badia-Paveze (ibid., fig. 4 en haut et fig. 5) ; 26. Villamarzana (Paltineri et Rubat Borel 2010, fig. $2, \mathrm{n}^{\circ} 25$ ) ; 27-29. Camaiore (ibid., fig. $2, \mathrm{n}^{\circ} 23,24$ et fig. 3 ).
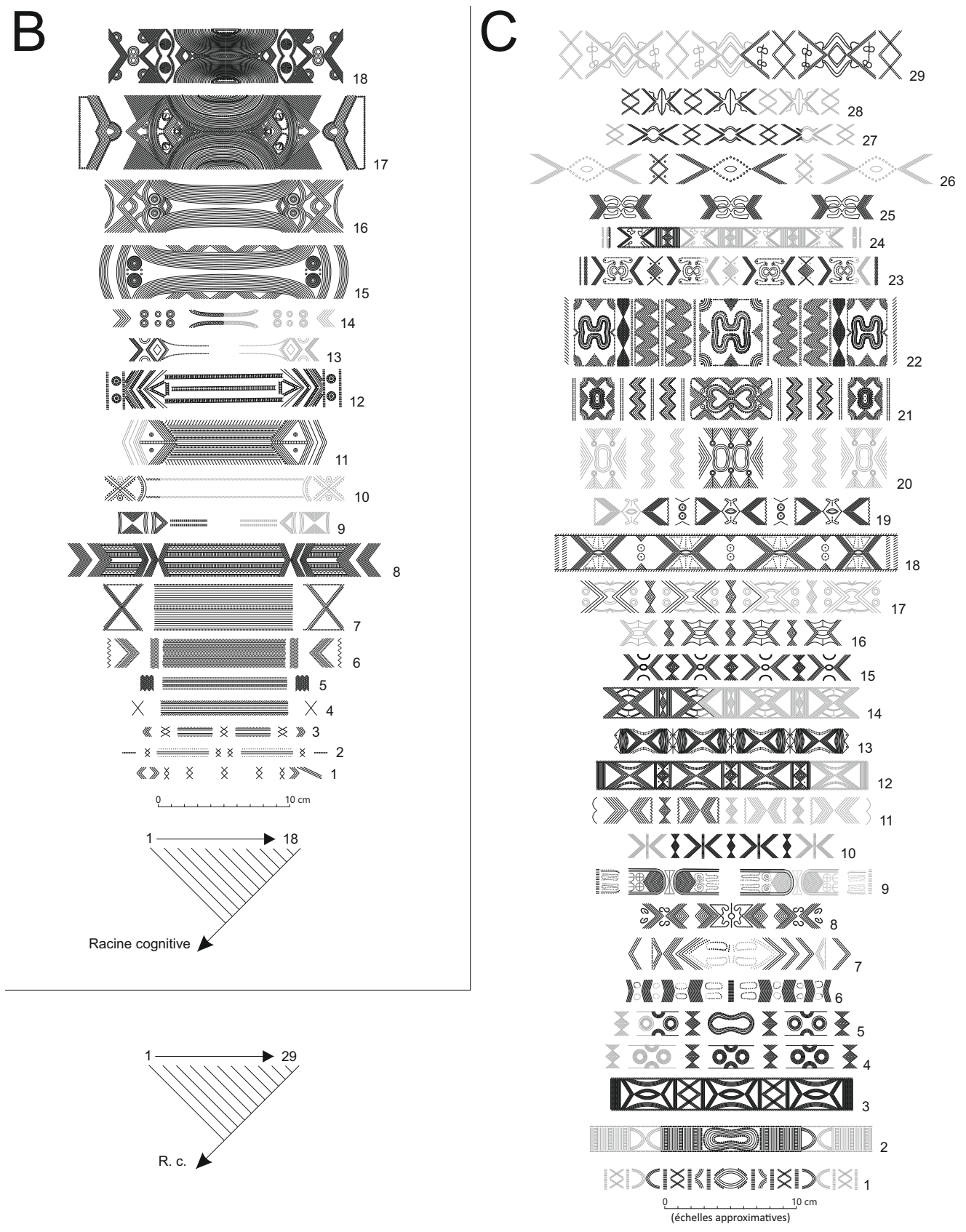
Figure 6 : Portions des décors sur bracelets à caractère présumé cosmogonique en vue orthogonale (sauf 11 : motifs d'une plaque de ceinture; 1 . Wisselsheim ( $c f$. fig. $\left.4, \mathrm{n}^{\circ} 15\right) ; 2$. Gross Bieberau (cf. fig. 4, $\left.\mathrm{n}^{\circ} 16\right)$; 3. Pfeddersheim (cf. fig. 4, $\left.\mathrm{n}^{\circ} 17\right)$; 4-6. Gross Bieberau (cf. fig. $4, \mathrm{n}^{\circ} 18,19$ et 21) ; 7-8. Lindenstruth (cf. fig. $4, \mathrm{n}^{\circ} 29$ et 30); 9. Provenance indéterminée (cf. fig. 4, $\mathrm{n}^{\circ} 37$ ) ; 10. Lauingen (cf. fig. 4, $\mathrm{n}^{\circ 37}$ ) ; 11. Allendorf (Uenze 1949$1950)$; 12. Savournon (Balac et Pages 1984, fig. 1, n¹) ; 13. Chiusa di Pesio (cf. fig. 5C, 5) ; 14. Cannes ou Cimiez [sur type Clans] (Lagrand 1968, pl. VII, n²); 15-16. Larnaud (cf. fig. 5C, 6 et 7) ; 17. Goncelin (cf. fig. 5C, 8) ; 18. Pezzana (cf. fig. 5C, 9) ; 19. Larnaud (cf. fig. 5C, 18) ; 20. Villa Nessi (cf. fig. 5C, 23) ; 21. Badia Paveze (cf. fig. 5C, 25) ; 22. Larnaud (cf. fig. 5C, 19) ; 23-25. Camaiore (cf. fig. 5C, 29, 28 et 27) ; 26-27. Borniga di Realdo (cf. fig. 5C, 1 et 2 ) ; 28. Monte Bignogne ( $c f$. fig. 5C, 20) ; 29-32. Cimiez 2 (cf. fig. 5C, 21 ; Mercurin et coll. 2011, fig. $7, \mathrm{n}^{\circ} 2$; ibid., fig. $6, \mathrm{n}^{\circ} 1 ; c f .5 C, 22$ ).

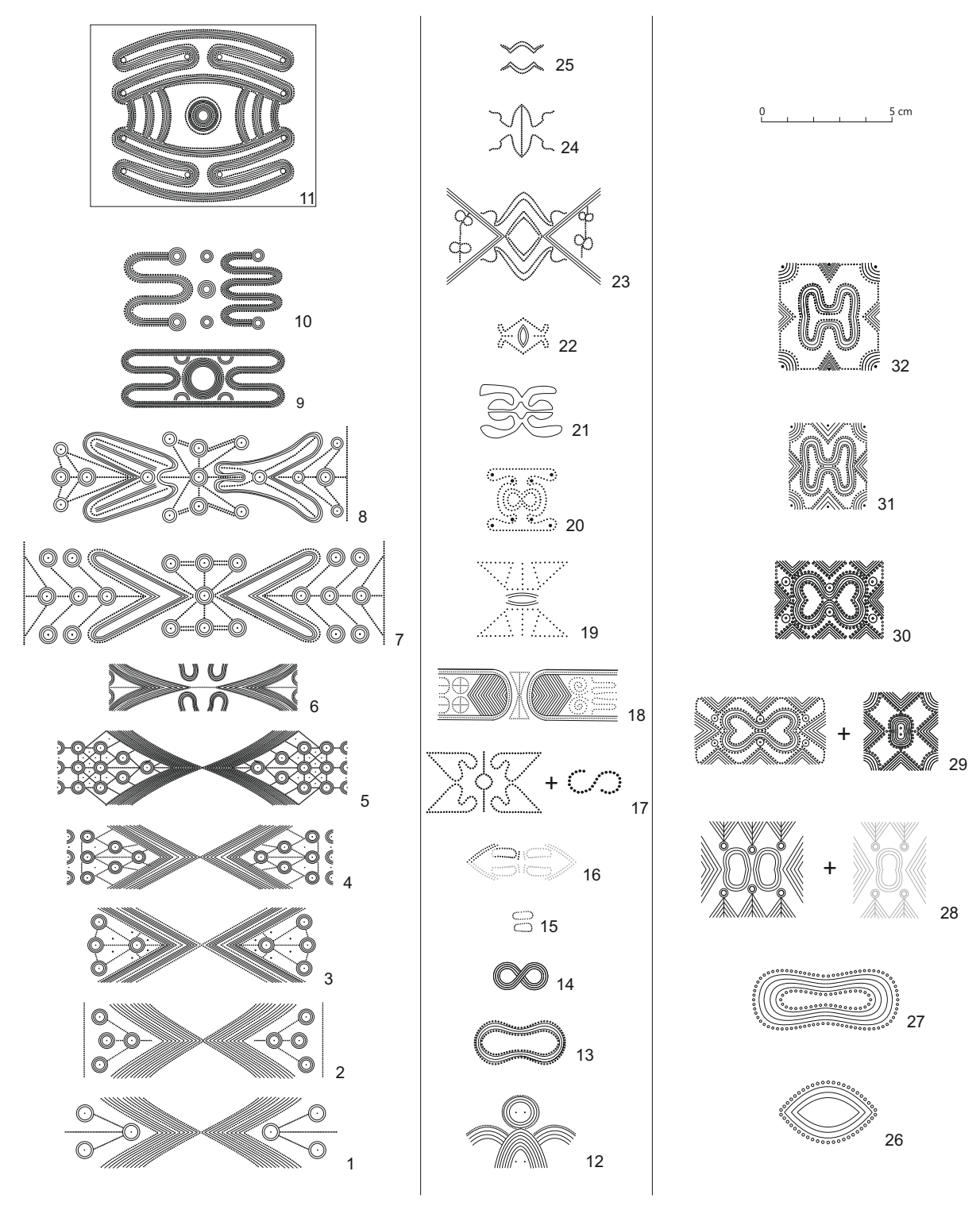


Figure 7: Seconde hypothèse de restitution du fragment textile de Santa Palomba (d'après Santis et al. 2010, fig. 2, D).

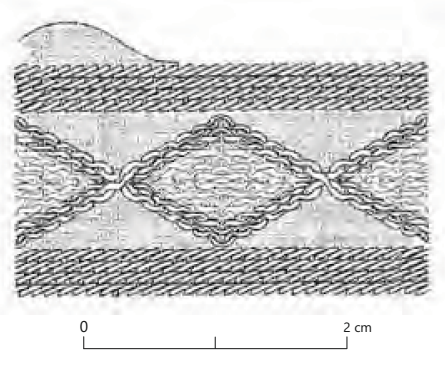

Figure 8 : Perspectives théoriques du système de parenté faisant le lien entre les porteurs d'épées et des porteuses des parures à graphismes complexes.

a : cas théorique $n^{\circ} 1$
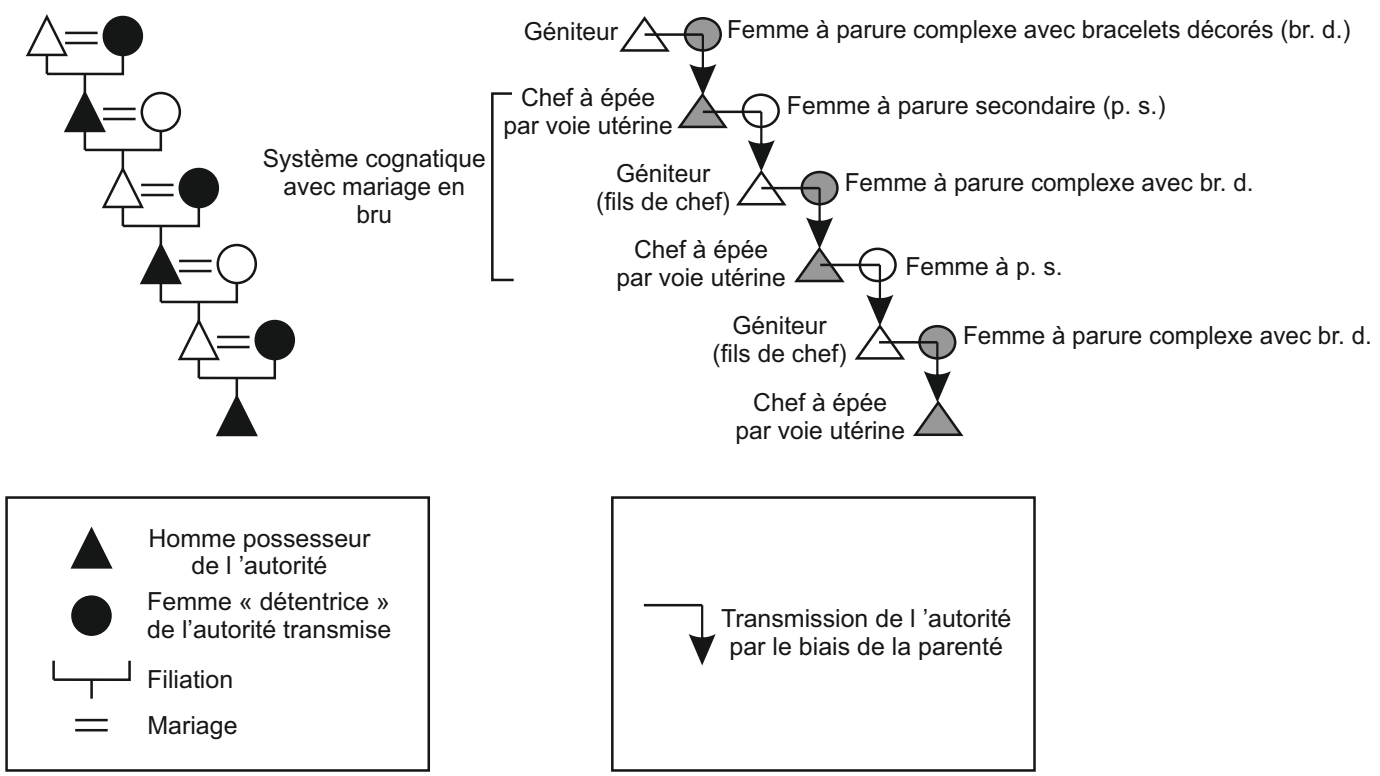

$b$ : cas théorique $n^{\circ} 2$
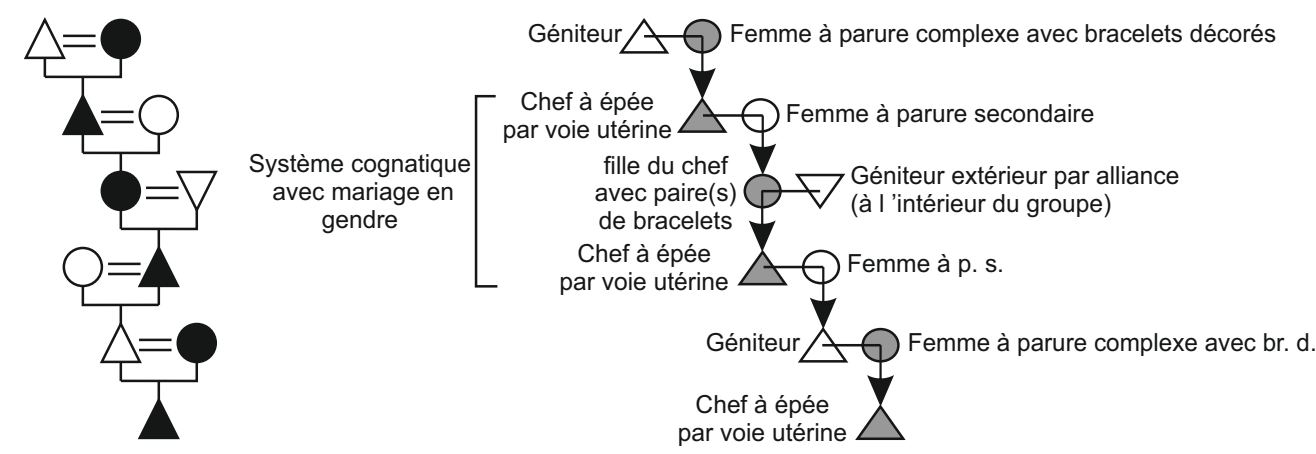
Figure 9: Sépulture triple de la nécropole de la Saulsotte avec les représentations orthonormées des décors sur bracelets (d'après Piette et al. 2005, fig. 7 et 8 ).

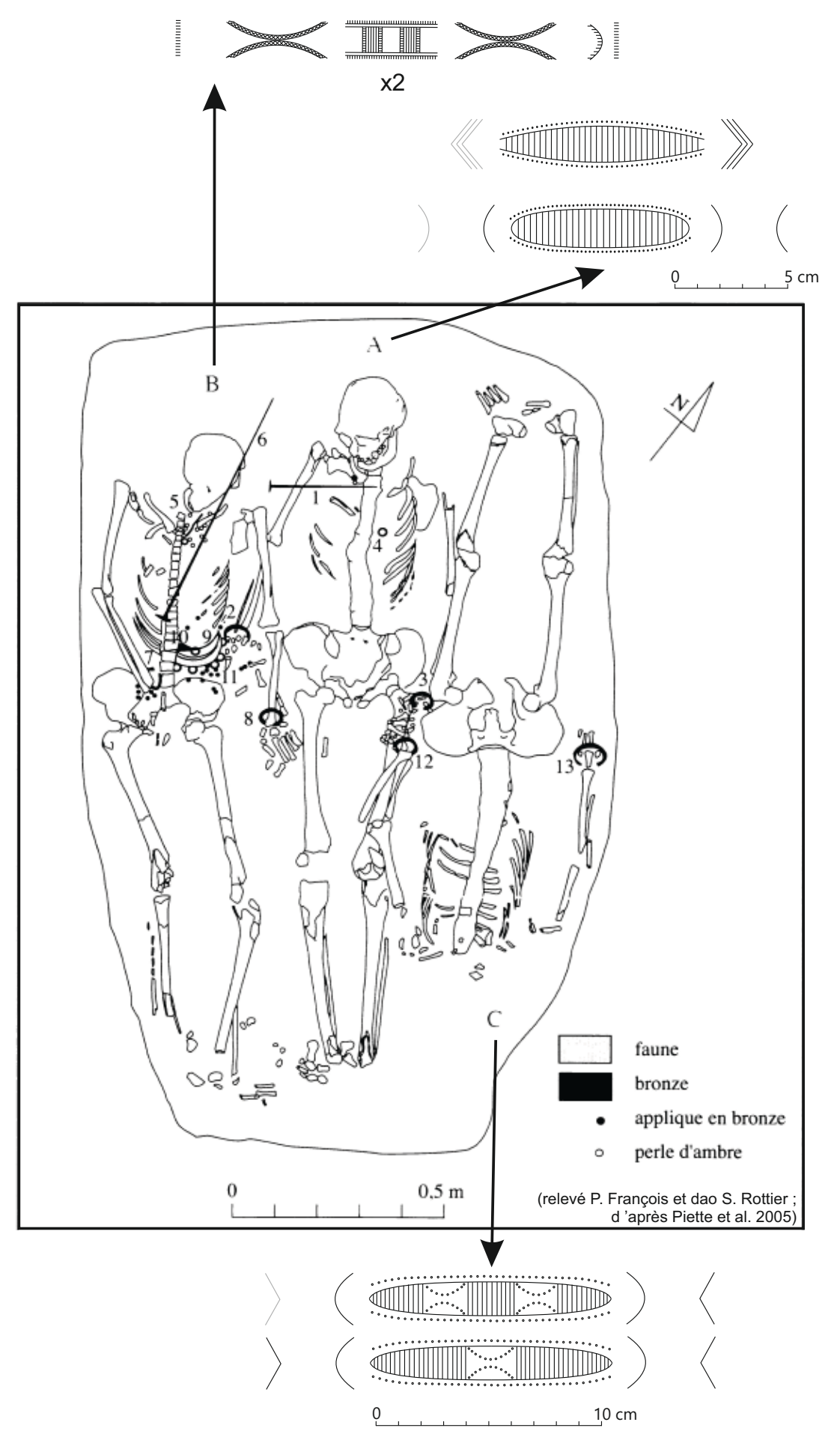




\title{
Clamor, couvre-chefs et batterie de cuisine : communication et cryptage des données chez les Celtes de l'âge du Fer
}

\author{
Olivier BUCHSENSCHUTZ \\ Directeur de recherches émérite au CNRS, \\ AOROC-Archéologie et Philologie d'Orient et d'Occident, CNRS / ENS, Paris

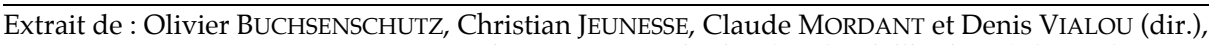 \\ Signes et communication dans les civilisations de la parole, Paris, \\ Édition électronique du CTHS (Actes des congrès des sociétés historiques et scientifiques), 2016. \\ Cet article a été validé par le comité de lecture des Éditions du CTHS dans le cadre de la publication \\ des actes du $139^{\mathrm{e}}$ Congrès national des sociétés historiques et scientifiques tenu à Nîmes en 2014.
}

\begin{abstract}
Résumé
Examinée à travers la rhétorique césarienne, la communication chez les Gaulois semble tantôt efficace, tantôt délibérément restreinte. César insiste sur la communication par le son, et insiste sur les limites de l'utilisation des documents écrits. On en a un peu rapidement déduit que les Gaulois n'avaient pas atteint le stade de l'écriture. Mais les nouvelles données archéologiques révèlent son utilisation plusieurs siècles avant la conquête romaine. $\mathrm{D}^{\prime}$ autres moyens de communication sont utilisés. Au premier âge du Fer, l'analyse des mises en scène funéraires révèle un jeu subtil entre la présence matérielle des composantes du banquet ou leur représentation sur des vases, entre le cadavre habillé et sa représentation en pierre. À partir du IV ${ }^{\mathrm{e}}$ siècle av. J.-C., le récit disparaît des images ; le banquet est représenté directement par la nourriture et les instruments de cuisine. Le décor des armes et des parures joue avec l'abstraction, la double lecture, et une symbolique réservée aux initiés.
\end{abstract}

Mots-clés : communication, celtes, écriture, messages sonores

\begin{abstract}
According to Caesar, communication with Gallic society seems to have been par efficient, part deliberately limited. Caesar insists on communication by sound and insists on the limited use of written documents. We have perhaps assumed to quickly that the Gauls did not use the written word, but new archaeological data reveals its use during the centuries before the Roman invasion. There were also other communication methods. During the Early Iron Age, funerary scenes reveal the subtle game between the actual presence of the components of the banquet and their depiction on vases, between a dressed corpse and its representation in stone. From the $4^{\text {th }}$ century BC the story telling disappears from the imagery as the banquet is directly represented by food and cooking implements. The décor on weapons and adornments becomes more abstract with a double meaning and a symbolic that is reserved only for the initiated. (traduction Rebecca Peake)
\end{abstract}




\section{Les moyens de communication des Gaulois d'après César: clamor et littera}

Nous avons tous en mémoire le texte dans lequel César s'étonne de la rapidité de la transmission des nouvelles chez le Gaulois, dans le De bello gallico, VII, 3 :

«Celeriter ad omnes Galliae civitates fama perfertur. Nam ubicumque maior atque illustrior incidit res, clamore per agros regionesque significant; hunc alii deinceps excipiunt et proximis tradunt, ut tum accidit. 3 Nam quae Cenabi oriente sole gesta essent, ante primam confectam vigiliam in finibus Arvernorum audita sunt, quod spatium est milium passuum circiter centum LX. »

« La nouvelle en parvient bientôt à toutes les cités de la Gaule ; car, dès qu'il arrive quelque chose de remarquable et d'intéressant, les Gaulois l'apprennent par des cris à travers les campagnes et d'un pays à l'autre. Ceux qui les entendent les transmettent aux plus proches comme on fit alors. En effet, la première veille n'était pas encore écoulée que les Arvernes savaient ce qui s'était passé à Cenabum au lever du soleil, c'est-à-dire à cent soixante milles environ de chez eux.»

Il cite toutefois un autre exemple pendant la Guerre $(\mathrm{V}, 53)$ chez les Rèmes :

«Interim ad Labienum per Remos incredibili celeritate de uictoria Caesaris fama perfertur, ut, cum ab hibernis Ciceronis milia passuum abesset circiter LX, eoque post horam nonam diei Caesar peruenisset, ante mediam noctem ad portas castrorum clamor oreretur, quo clamore significatio uictoriae gratulatioque ab Remis Labieno fieret. »

«Cependant le bruit de la victoire de César fut porté à Labienus, chez les Rèmes, avec une si incroyable vitesse que, bien qu'éloigné de soixante mille pas des quartiers de Cicéron, où César n'était arrivé qu'après la neuvième heure du jour, des acclamations s'élevèrent aux portes du camp avant minuit, et que déjà, par leurs cris de joie, les Rèmes félicitaient Labienus de cette victoire. »

La comparaison des deux textes révèle une grande similarité du vocabulaire, le lecteur a l'impression que l'auteur a fait un copié/collé. On retrouve en effet les termes de clamor, celeriter, fama. Une exploration systématique du texte permet de retrouver fama employée dans le même sens, une nouvelle qui sort de l'ordinaire. Clamor, employé 24 fois, désigne soit le tumulte d'une bataille, soit, comme ici, le moyen de transmettre une information. Il s'oppose à littera, qui est un message écrit utilisé par les Romains, comme on le voit par exemple dans le livre V, 45 et 46 : Cicéron a envoyé une lettre confiée à un Gaulois, qui peut passer à travers les lignes ennemies sans encombre, et César lui envoie également un message écrit :

[5,45] Quanto erat in dies grauior atque asperior oppugnatio, et maxime quod magna parte militum confecta uulneribus res ad paucitatem defensorum peruenerat, tanto crebriores litterae nuntiique ad Caesarem mittebantur; quorum pars deprehensa in conspectu nostrorum militum cum cruciatu necabatur. Erat unus intus Neruius nomine Vertico, loco natus honesto, qui a prima obsidione ad Ciceronem perfugerat suamque ei fidem praestiterat. Hic seruo spe libertatis magnisque persuadet praemiis, ut litteras ad Caesarem deferat. Has ille in iaculo illigatas effert et Gallus inter Gallos sine ulla suspicione uersatus ad Caesarem peruenit. Ab eo de periculis Ciceronis legionisque cognoscitur. »

«Plus le siège devenait rude et difficile à soutenir, surtout avec le peu de défenseurs auxquels nous réduisait chaque jour le grand nombre des blessés, plus Cicéron dépêchait vers César de courriers porteurs de ses lettres ; la plupart étaient arrêtés et cruellement mis à mort à la vue de nos soldats. Dans le camp était un Nervien, nommé Vertico, d'une naissance distinguée, qui, dès le commencement du siège, s'était rendu près de Cicéron et lui avait engagé sa foi. II détermine un de ses esclaves, par l'espoir de la liberté et par de grandes récompenses, à porter une lettre à César. L'esclave la porte, attachée à son javelot, et, Gaulois lui-même, il se mêle aux Gaulois sans inspirer de défiance, et arrive auprès de César, qu'il instruit des dangers de Cicéron et de la légion. » 
$[5,46]$ Caesar acceptis litteris hora circiter XI diei statim nuntium in Bellouacos ad M. Crassum quaestorem mittit, cuius hiberna aberant ab eo milia passuum XXV; iubet media nocte legionem proficisci celeriterque ad se uenire. Exit cum nuntio Crassus. Alterum ad Gaium Fabium legatum mittit, ut in Atrebatium fines legionem adducat, qua sibi iter faciendum sciebat. Scribit Labieno, si rei publicae commodo facere posset, cum legione ad fines Neruiorum ueniat. Reliquam partem exercitus, quod paulo aberat longius, non putat exspectandam; equites circiter quadringentos ex proximis hibernis colligit. »

«César, ayant reçu cette lettre vers la onzième heure du jour, envoie aussitôt un courrier au questeur M. Crassus, dont les quartiers étaient chez les Bellovaques, à vingt-cinq mille pas de distance. Il lui ordonne de partir au milieu de la nuit avec sa légion et de venir le joindre en toute hâte. Crassus part avec le courrier. Un autre est envoyé au lieutenant C. Fabius, pour qu'il conduise sa légion sur les terres des Atrébates, qu'il savait avoir à traverser luimême. Il écrit à Labienus de se rendre, s'il le peut sans compromettre les intérêts de la République, dans le pays des Nerviens avec sa légion. Il ne croit pas devoir attendre le reste de l'armée qui était un peu plus éloigné, et rassemble environ quatre cents cavaliers des quartiers voisins. »

Vercingétorix utilise aussi des messagers, par exemple dans le livre VII, 8, qui lui annoncent que César a passé les Cévennes :

«Celeriter haec fama ac nuntiis ad Vercingetorigem perferuntur. »

« La renommée et des courriers en informent bientôt Vercingétorix. »

Ainsi selon le témoignage de César, les Gaulois n'utilisent pas de messages écrits. La transmission des informations se fait par des messagers ou, quand la nouvelle est importante et urgente, par un bruit (clamor) qui est relayé de village en village. On imagine qu'il s'agit de cris, de "tam-tam», des messages codés sonores plus faciles à percevoir à distance que le langage courant. Il faudrait chercher sans doute des comparaisons chez les populations qui utilisaient dans plusieurs régions du monde une langue sifflée pour se parler d'une colline à l'autre, avec une portée qui pouvait aller jusqu'à dix kilomètres, les habitants des îles Canaries par exemple, de Madagascar ou encore les bergers de Pyrénées (Arripe 1984 ; fr.wikipedia.org/wiki/Langage_sifflé) ou de Suisse (le yodel).

Cela peut nous sembler extraordinaire aujourd'hui, parce que notre environnement est rempli de bruits parasites depuis le développement des moteurs. Ceux qui ont eu l'expérience d'une marche en Afrique savent que dans cette ambiance, le moindre bruit est perceptible à longue distance et facilement interprétable: on peut distinguer un message transmis par des humains, des bruits discrets du vent ou des animaux. Personnellement, je pencherais plus pour une langue sifflée ou codée que pour le tamtam, qu'éventuellement César aurait pu citer, et pour lequel nous n'avons aucun témoignage archéologique.

\section{Représentation ou mise en scène dans les sépultures}

La plus grande partie des vestiges archéologiques dont nous disposons pour l'âge du Fer sont des déchets, qui nous donnent une vision objective, mais limitée à la vie quotidienne et à l'économie des populations. Heureusement les innombrables et souvent riches sépultures sont de véritables mises en scène volontaires. Elles portent un message que nous sommes encore loin de comprendre dans le détail. Elles constituent une expression de la piété de l'entourage du défunt, à travers le traitement du corps, son vêtement, ses insignes ou sa panoplie, qui traduisent sa place dans la société. Souvent des vestiges culinaires au sens large renvoient à l'évocation d'un banquet, d'une cérémonie funéraire, plutôt qu'à un viatique pour la survie du défunt. 


\section{Les coiffures (fig. 1)}

Plusieurs chapeaux, dont nous avons la trace à la fois dans des sépultures et dans des sculptures en pierre, présentent des formes aussi complexes que des mitres d'évêques ou des bicornes de polytechniciens (Dossiers de l'archéologie, $\mathrm{n}^{\circ} 329$, sept.-oct. 2008). Dans la tombe de Hochdorf (Wurtemberg) (Biel 1985, fig.33) comme dans celle de Mardié (Loiret) (Verger et al. 2002, fig. 4), la tête des défunts est coiffée d'un chapeau conique en écorce de bouleau qui renvoie à une sculpture en pierre découverte sur un tertre funéraire à Hirschlanden (Wurtemberg) (Zürn 1964 ; Frey 2008, p. 32 et p. 65). Les fouilles du Glauberg (Hesse) ont livré une sculpture en pierre et des fragments de trois autres associées à une sépulture dans laquelle se retrouvent les éléments figurés, les armes comme le costume et les parures. Le plus frappant est une coiffe en cuir montée sur une armature de métal, clairement représentée sur les sculptures. Elle couvre le sommet du crâne du personnage et déploie des deux côtés de sa tête de grandes oreilles qui font évidemment penser aux oreilles de Mickey Mouse (Herrmann 2008, p. 29, 61). Avant cette découverte, on pensait que ces «vessies de poisson" ou "feuilles de gui » appartenaient au décor des objets, ou à une symbolique indépendante des rares représentations humaines et animales qui émergeaient d'éléments abstraits. Elles renvoient selon toute vraisemblance à la fonction du défunt. Il serait téméraire d'identifier cette fonction, surtout pour une période aussi ancienne de l'âge du Fer pour laquelle aucune source ne décrit les fonctions des personnes éminentes, et encore moins les signes qui les distinguent du peuple. Mais il nous semble clair qu'il y a là un signe de la fonction du défunt limpide pour les contemporains.

\section{Les banquets funéraires}

Deux supports sont utilisés pour exprimer la nature et la richesse du banquet. En Europe centrale et orientale, des vases en bronze décorés de frises narratives illustrent avec précision des cortèges de différentes catégories de personnages apportant des cadeaux ou le signe de leur allégeance à des aristocrates qui se défient dans des duels sportifs, banquettent, ou encore s'accouplent. Par exemple, la situle de Kuffarn déroule sa frise comme une bande dessinée, un véritable récit déposé dans la sépulture (Frey 1962, Urban 1989, p. 198-199) (fig. 2).

À l'ouest, ce sont les objets du banquet ou du foyer qui sont réunis et mis en scène dans la chambre funéraire, à la place de leur représentation plastique. C'est le cas particulièrement dans la tombe de Hochdorf, où le mort est accompagné non seulement d'un char de cérémonie, d'une kliné en bronze, mais aussi d'un service à boire et à manger pour huit convives (Biel 1985) (fig. 3). Il est coiffé d'un chapeau conique en écorce de bouleau.

À la fin de l'âge du Fer, les sépultures les plus importantes sont disposées dans des chambres funéraires plus modestes, de trois à cinq mètres carrés. Le corps, souvent incinéré, est déposé sur le sol un peu à l'écart, alors que les éléments du banquet, des chenets ou des grills, occupent la place d'honneur au centre de la chambre funéraire (fig. 4). Des demi-carcasses, des vases contenant une mince couche de denrées au sommet de leur remplissage, sont disposés astucieusement pour en imposer au spectateur du banquet ou des funérailles, en simulant une grande abondance de dons (Bouche et al. 2002 ; Meniel et Metzler 1999) (fig. 4). 


\section{Le cryptage des données}

Les décors des objets du premier âge du Fer étaient soit géométriques et abstraits, soit explicites. On y reconnaît des animaux, des hommes, même des scènes narratives comme celles que nous avons évoquées plus haut. À partir de l'époque de La Tène, la narration disparait complètement, les personnages sculptés ou gravés dans la pierre ou le bronze sont peu nombreux, ils sont rarement représentés en train d'exercer une activité ; ils disparaissent peu à peu à La Tène moyenne au profit d'enchevêtrements et de motifs abstraits. Nous avons tendance à lire comme un assemblage de motifs décoratifs ces représentations, mais certains indices semblent montrer qu'elles délivraient pour les Celtes un message plus ou moins explicite. Il est très difficile toutefois de le décrypter.

Non seulement nous manquons d'une épopée celtique qui pourrait nous donner des clés d'interprétation, - les légendes celtiques médiévales sont bien loin des Celtes de l'Antiquité -, mais on peut parfois se demander si les sculpteurs n'ont pas volontairement crypté les messages qui sont évoqués dans les objets qu'ils ont produits. Les transformations du motif universel du maître des animaux ou de l'arbre de vie emprunté à l'Orient sont significatives. Sur l'épée de Port, un poinçon reproduit fidèlement la scène (Buchsenschutz 2007, fig. 30); sur une fibule du Parsberg, deux animaux fantastiques encadrent une tête aux yeux exorbités (Kruta 2000, fig. 135); $\mathrm{V}$. Kruta reconnaît le même thème sur un motif triangulaire abstrait d'un casque de Monte Bibele (ibid., fig. 133) (fig. 5). Des constructions savantes, sur un schéma géométrique très complexe, entremêlent des motifs curvilignes dont émerge parfois une tête de monstre. Faut-il y voir une influence pythagoricienne, un simple exercice décoratif, ou l'expression d'une cosmogonie locale? Les arguments en faveur de l'une ou l'autre hypothèse manquent cruellement.

En analysant de très près deux fourreaux décorés découverts respectivement à EpiaisRhus dans le Val-d'Oise, et à Moscano di Fabriano près d'Ancône, André Rapin a pu montrer que certains reliefs avaient été exécutés avec le même poinçon (Rapin 2003, fig. 2) (fig. 6). En revanche, l'emploi d'un poinçon au décor inversé sur une des onze bandes qui ornent ce fourreau reste une énigme, d'autant plus qu'elle est à peine visible.

Peut-on parler d'une écriture codée ? Cette abstraction, et en même temps le soin apporté à la réalisation de ces motifs et de ces compositions complexes, peuvent le laisser penser, mais on manque de confirmation pour valider ces hypothèses.

Je voudrais insister en conclusion sur les conditions dans lesquelles se trouvaient les Celtes et toutes les populations dans l'Antiquité par rapport à l'écriture et dans un milieu où les bruits étaient rares et les hommes relativement silencieux. Il faut se représenter un monde dans lequel le moindre son peut être immédiatement interprété. Dans la Gaule en guerre, les signaux sonores atteignent leur but plus vite et plus sûrement que les messages écrits, comme en témoigne lui-même César. L'utilisation de l'écriture par les Gaulois n'est pas pour autant négligeable, dans le monde des comptages et du commerce. Elle ne concerne ni les sciences religieuses, ni surtout les récits mythologiques, qui nous manquent pour comprendre les autres messages laissés par les Celtes. Les rituels funéraires, les armes et les parures, représentent certainement quelque chose de précis pour les contemporains, mais leur signification nous échappe. Le choix d'un vocabulaire artistique de plus en plus abstrait, voire délibérément ésotérique, satisfait notre œil, mais le message devient pour nous totalement incompréhensible. On peut mettre en parallèle le rire des Celtes devant les représentations anthropomorphes des dieux de Delphes avec leur peu d'intérêt pour l'écriture. Il s'agit d'un choix culturel délibéré. 


\section{Bibliographie}

Dossiers de l'archéologie, $\mathrm{n}^{\circ}$ 329, sept.-oct. 2008. Contributions traduites en français de plusieurs spécialistes de ces questions, O.-H. Frey, R. Herrmann, S. Verger, Ch. Peyre, réunissant une partie de l'abondante bibliographie.

Jules César, Guerre des Gaules, texte et traduction par L.-A. CONSTANS, 2 volumes, Les Belles Lettres, Paris, 1978, 1972.

http:// fr.wikipedia.org/wiki/Langage_sifflé

ARRIPE R. (1984). Les siffleurs d'Aas. Imprimerie de la Monnaie, Pau.

BIEL J. (1985). Der Keltenfürst von Hochdorf. Theiss, Stuttgart.

Bouche K., BlancQuaert G., GinOuX N. (2002). Les fouilles archéologiques de l'ensemble funéraire aristocratique d'époque gauloise de Raillencourt-Sainte-Olle (Nord), Actes des $X^{\mathrm{e}}$ journées Lunula, Bruxelles-février 2002, Archaelogia protohistorica, X, Bruxelles, p. 74-79.

Buchsenschutz O. (2007). Les Celtes de l'âge du Fer. Armand Colin, Paris.

Buchsenschutz O., Chardenoux M.-B., Gruel K., LAmbert P.-Y., Lejars T., VerGer S. (2015), L'Europe celtique à l'âge de Fer (VIII'-1 $I^{e r}$ siècles) Nouvelle Clio, PUF, Paris.

FREY O. H. (1962). Die Situla von Kuffarn, ein figürlich verzieter Bronzeblecheimer der Zeit um 400 v. Chr., Veröffentlichungen aus dem Naturhistorischen Museum in Wien, Neue Folge, p. $1-12$.

FREY O. H. (2008). Hommes ou héros ? Les statues du Glauberg et la grande sculpture chez les Celtes anciens, Les Dossiers de l'Archéologie, n 329, octobre 2008, p. 64 - 71.

HERRMANN F. R. (2008). Le Glauberg, résidence princière, tombes princières et sanctuaire, Les Dossiers de l'Archéologie, n 329, octobre 2008, p. 18-35.

KRUTA V. (2000). Les Celtes, Histoire et dictionnaire, Bouquins, Robert Laffont, Paris.

MetZler J. et N., MÉNIEL P. (1999). Lamadelaine, une nécropole de l'oppidum du Titelberg. Dossiers d'archéologie du Musée national d'Histoire et d'Art, VI, Luxembourg.

RAPIN A. (2003). Les analyses sémiologiques de l'image : l'iconographie du deuxième âge du Fer, in O. BuCHSENSCHutZ, A. BulaRD, M.B. ChaRdenOuX, N. GinOuX (dir.), L'âge du Fer en Ile-de-France - Décors, images et signes de l'âge du Fer européen, Actes du XXVIe Colloque de l'AFEAF, Paris-Saint-Denis, 24e et 26e suppléments à la Revue Archéologique du Centre de la France, p. 49-62.

URBAN O. (1989). Wegweiser in die Urgeschichte Österreichs, ÖsterreichischerBundesverlag, Vienne.

Verger St., Milcent P.-Y., Moulherat Chr. (2002). La Butte Moreau à Mardié (Loiret) et les tombes aristocratiques du Centre de la Gaule au V $V^{\mathrm{e}}$ siècle avant J.-C., in D. MARANSKI, et V. GUICHARD (ed.), Les âges du Fer en Nivernais, Bourbonnais et Berry oriental: regards européens sur les âges du Fer en France, actes du XVII ${ }^{\mathrm{e}}$ Colloque de l'AFEAF, Nevers, 20-23 mai 1993, Bibracte 6, Glux-en-Glenne, p. 117-150.

ZÜRN H. (1964). Eine hallstattzeitliche Stele von Hirschlanden, Kr. Leonsberg (Württemberg), Vorbericht, Germania, 42, p. 27-36. 


\section{Illustrations}

Figure 1: Les coiffures (d'après Services archéologiques de Hesse et de BadenWürtemberg).

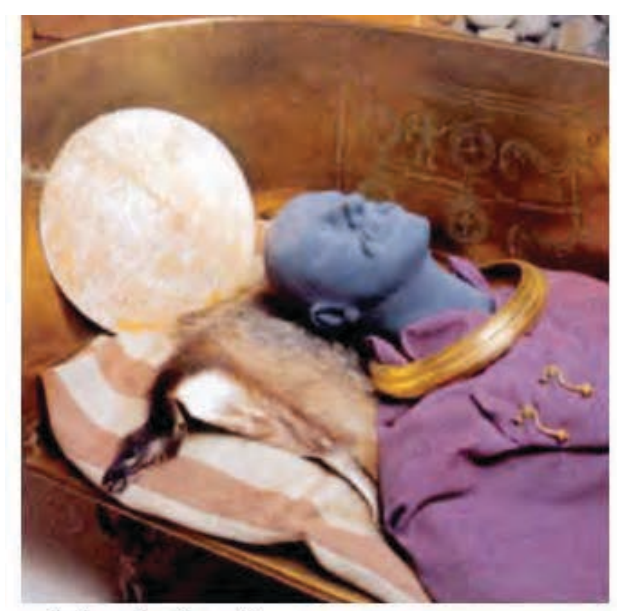

Hochdorf

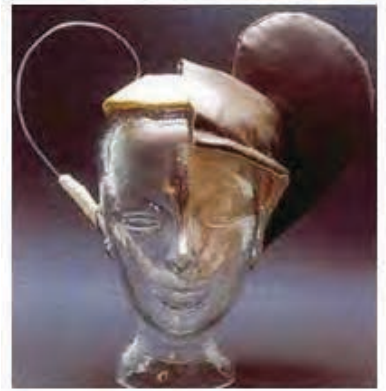

Coiffe de la tombe du Glauberg

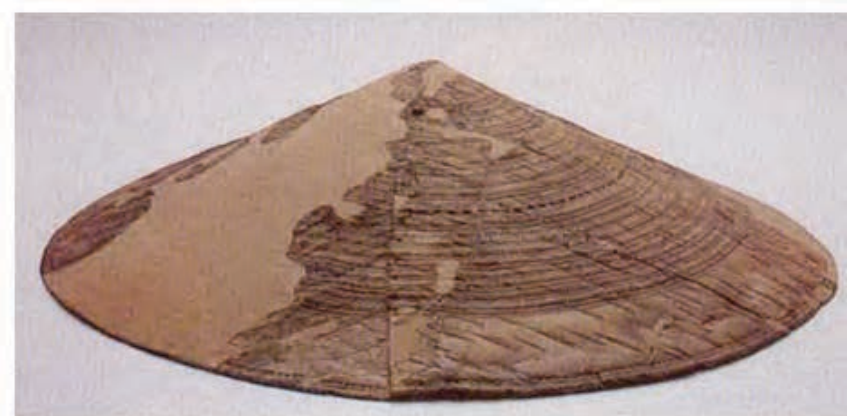

Chapeaux d'écorce Hochdorf

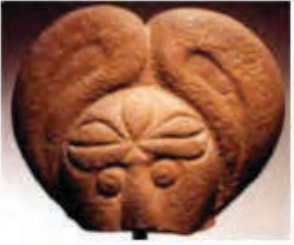

Tête de Heidelberg, Musée de Karlsruhe

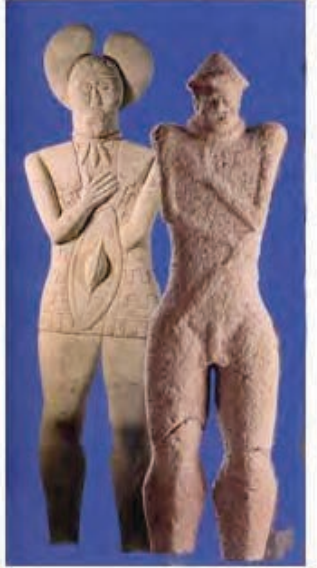

Sculptures du Glauberg et de Hirschlanden VIe s 
Figure 2 : Décoration de la situle de Kuffarn (d'après Urban 1989, p. 198-199).
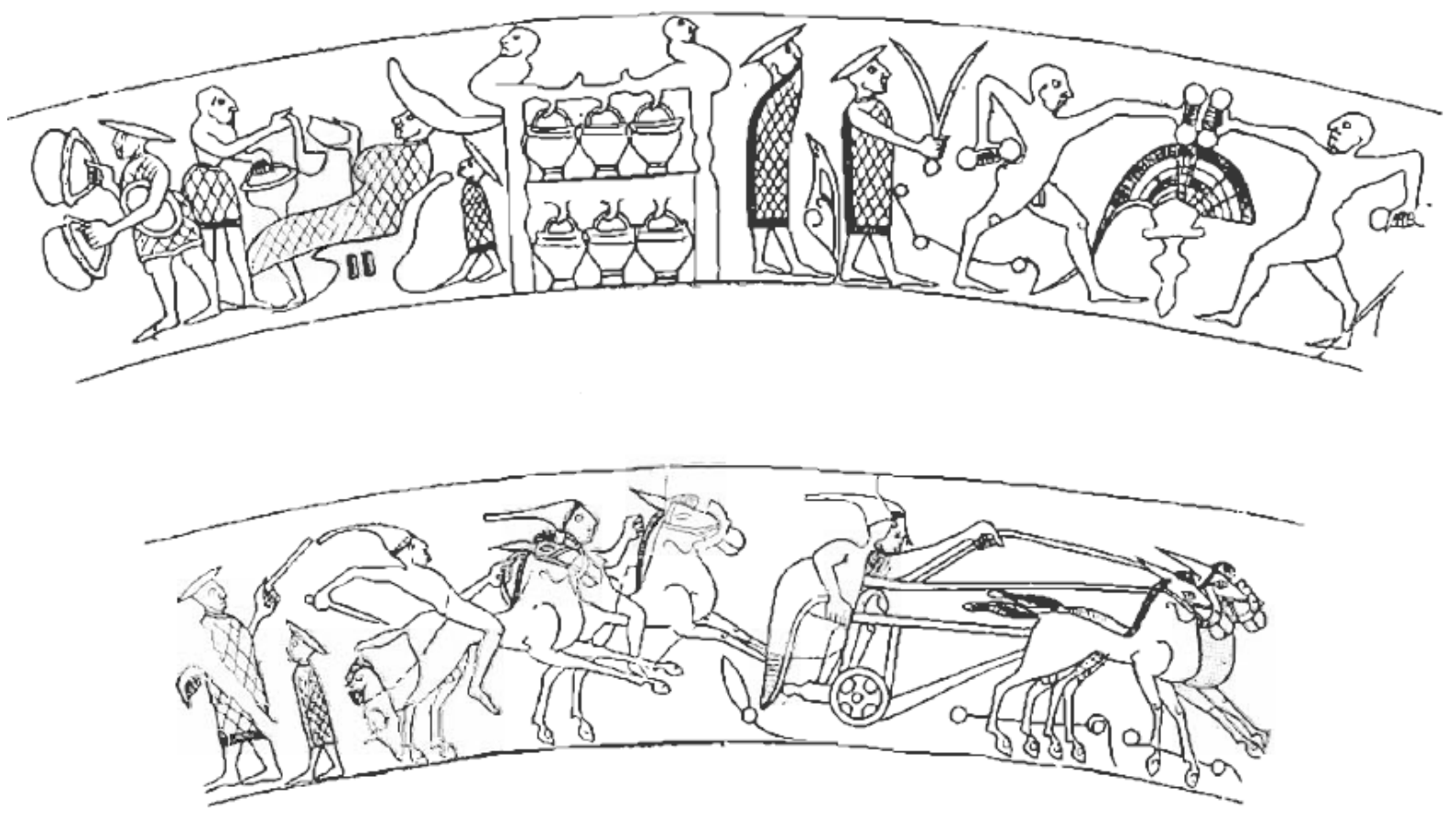

Figure 3. La tombe de Hochdorf (Wurtemberg) (d'après J. Biel 1985, fig. 33).

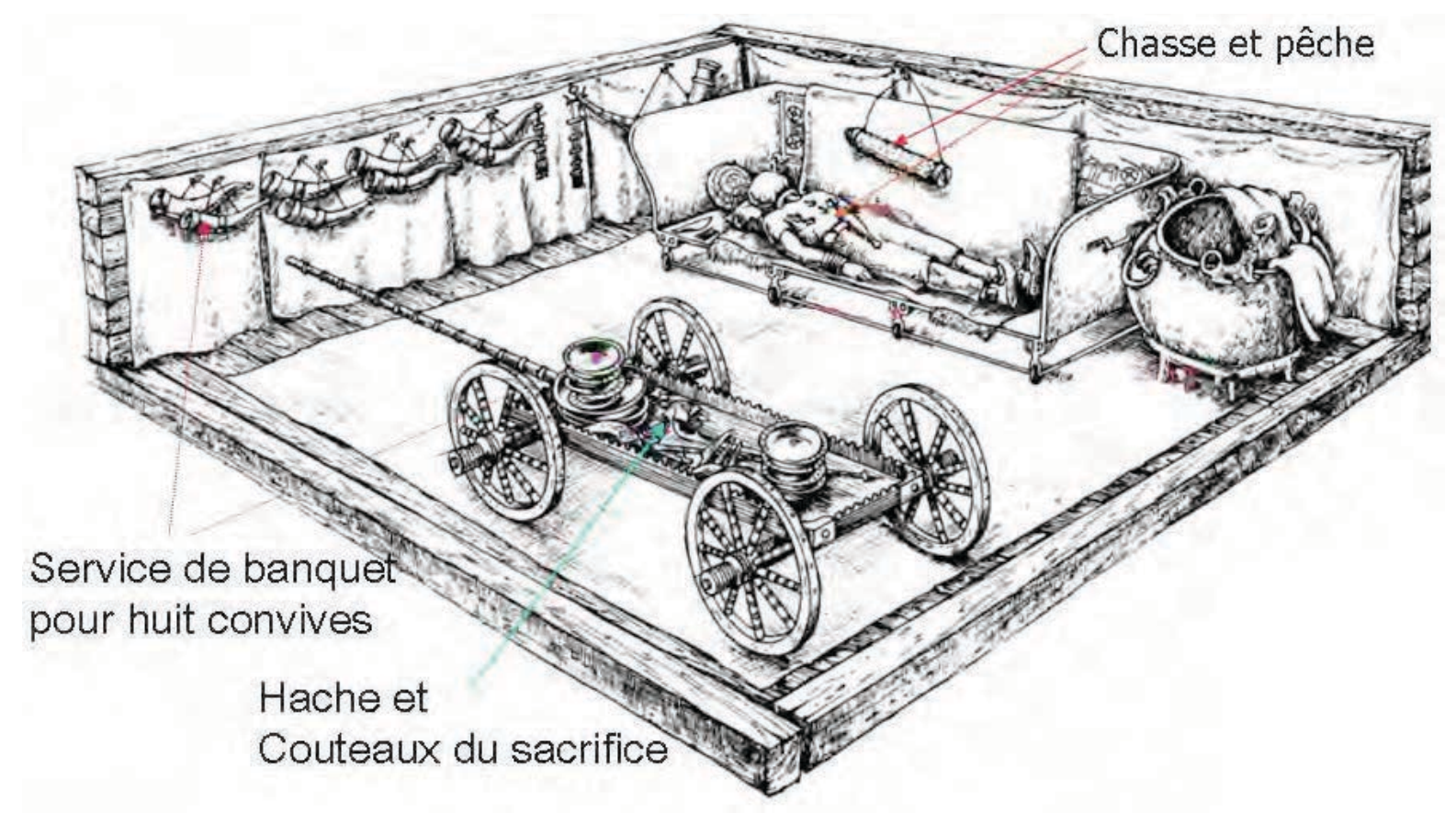


Figure 4. Chenets au centre de la tombe de Raillencourt-Saint-Olle (Nord) (140-80 av. J. C.) (Fouille et cliché K. Bouche).

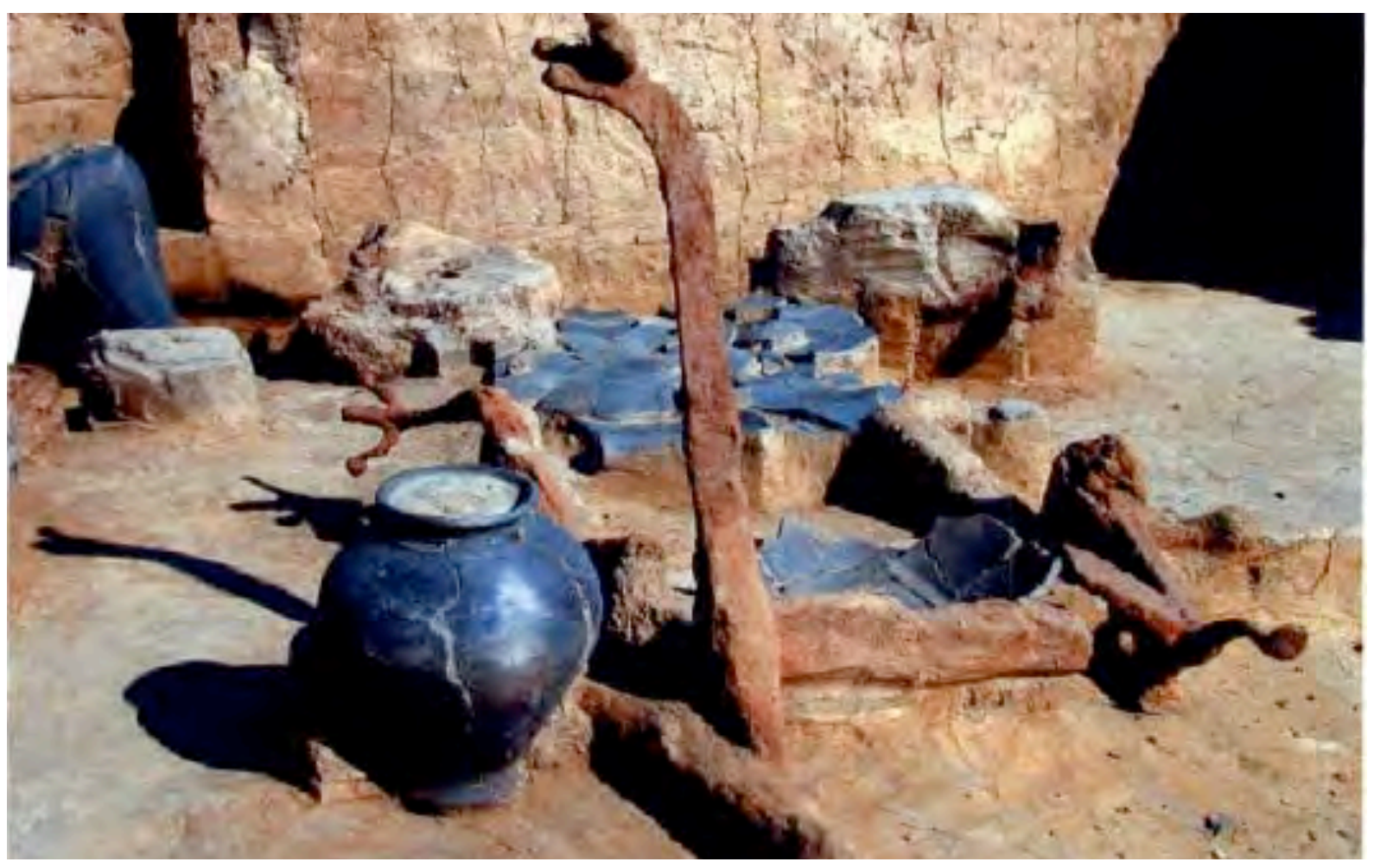

Figure 5. Épée de Port, arbre de vie; Parsberg fibule à masque; Monte Bibele, casque (d'après V. Kruta 2000, fig.133, 135 et O. Buchsenschutz 2007, fig. 30).
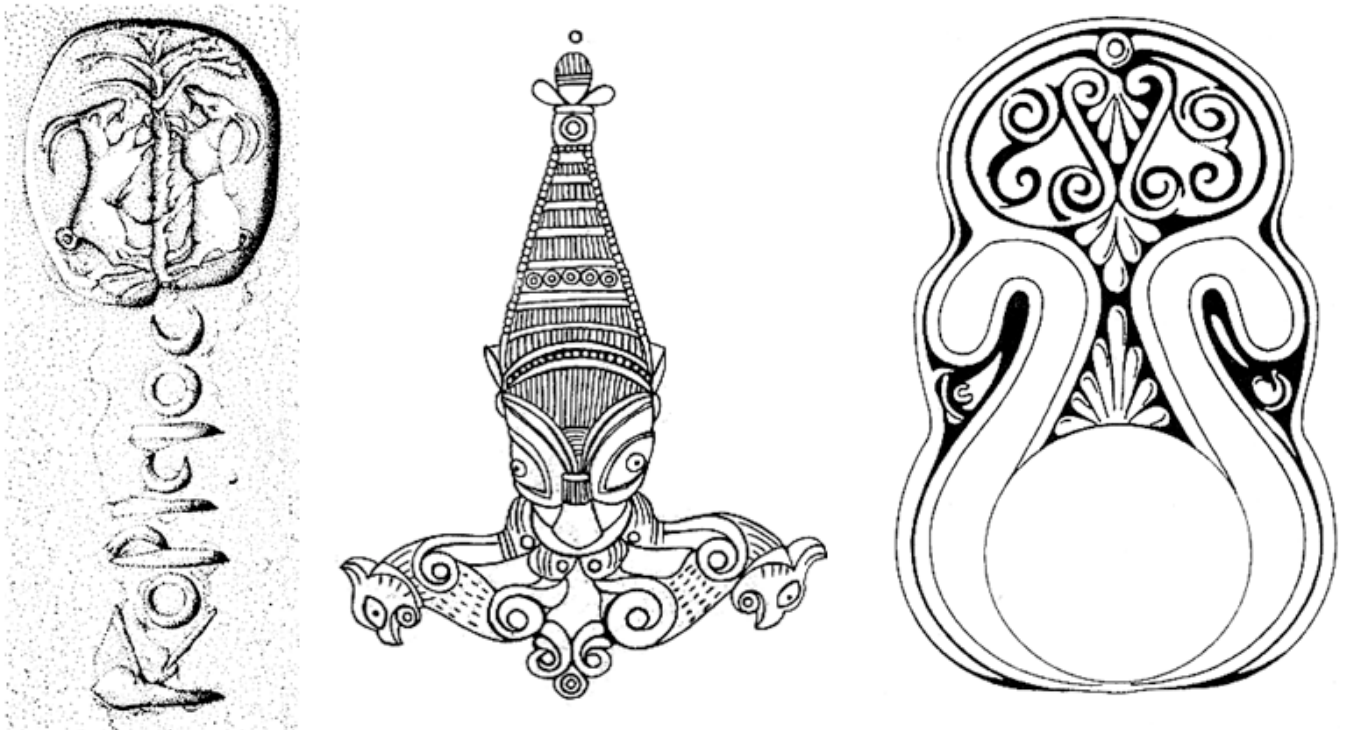
Figure 6. Fourreaux décorés: Moscano, Epiais-Rhus, Fillotrano, Flavigny (d'après A. Rapin 2003, fig. 2).
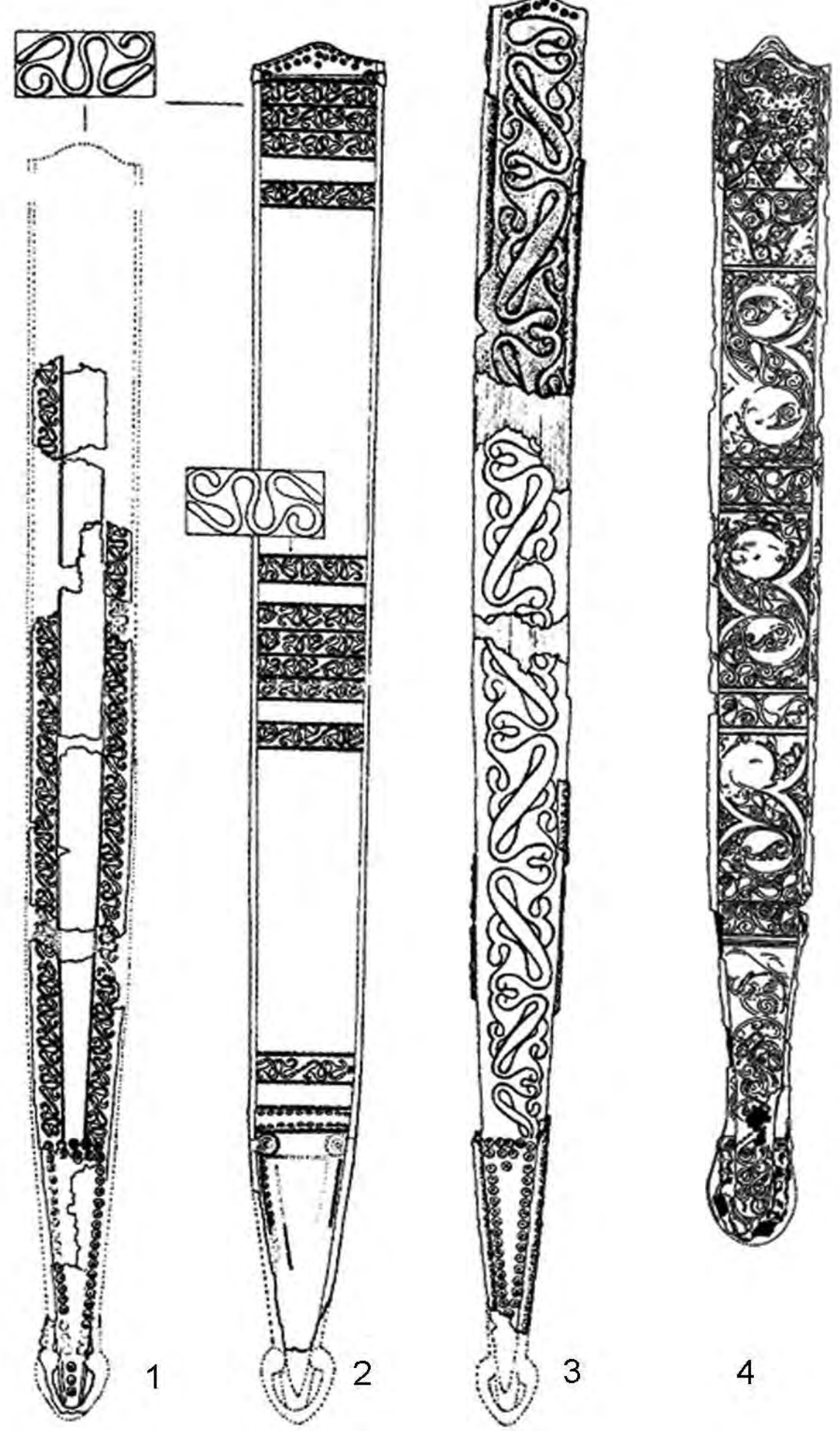


\title{
La dalle gravée de Teyssieu (Lot), support d'une communication sans écriture
}

\author{
Gilbert-Robert DELAHAYE \\ Président de la Société des fouilles archéologiques \\ et des monuments historiques de l'Yonne

\begin{abstract}
Extrait de : Olivier BuCHSENSCHUTZ, Christian JeunESSE, Claude MORDANT et Denis VialOU (dir.), Signes et communication dans les civilisations de la parole, Paris, Édition électronique du CTHS (Actes des congrès des sociétés historiques et scientifiques), 2016.
\end{abstract} \\ Cet article a été validé par le comité de lecture des Éditions du CTHS dans le cadre de la publication \\ des actes du $139^{\text {e }}$ Congrès national des sociétés historiques et scientifiques tenu à Nîmes en 2014.
}

\begin{abstract}
Résumé
À Teyssieu (Lot), une dalle, approximativement circulaire, gravée sur une de ses faces d'un tablier de jeu de trictrac, comportant des cupules, aurait été réemployée pour indiquer, par le dépôt d'un caillou dans telle ou telle cupule, le lieu où le pâtre menait les animaux qu'il prenait en charge. Si la dernière fonction de cette pierre s'est transmise localement par voie orale, les sites auxquels correspondaient les cupules creusées dans la pierre ne sont plus connus. Bien que constituant une sorte de document cartographique, celui-ci n'est pas réaliste du fait de la première utilisation de la gravure. On ne sait qui, parmi la population, connaissait l'exacte signification de cette forme de communication. On ignore aussi le lieu où était exposée cette dalle et l'époque où elle fut utilisée, sans doute avant la généralisation de l'alphabétisation. Elle constitue, quoi qu'il en soit, une forme de communication sans écriture et des plus originales.
\end{abstract}

Mots-clés: Dalle circulaire, cupules, jeu de trictrac, pâtre, lieux de pâture.

\begin{abstract}
A sub-circular stone slab found in Teyssieu (Lot) has an engraving of a backgammon board with small cavities on one of its sides. This board was reused by shepherds as the cavities filled with stones indicated the place where the animals were kept. Even though the last known use of this stone has been passed on orally, the knowledge of the actual places represented by each cavity has been lost. A sort of map, it is not very realistic due to the stone's first use as a game. It is also not known who in the community understood the significance of this form of communication or where and when the stone slab was displayed, though probably before widespread literacy. It represents an original form of communication without writing. (traduction Rebecca Peake)
\end{abstract}

Il est généralement admis que la communication est le moyen de transmettre de l'information à l'aide d'une écriture, de sons, de signes, de gestes et, de nos jours, le plus souvent d'images, comme c'est le cas avec la télévision et le cinéma. Par le passé, néanmoins, d'autres moyens de communication ont aussi existé, des moyens parfois plus ou moins cryptés, qui, par la position d'un objet, par exemple, renseignaient des interlocuteurs prévenus d'un état de fait ou d'une situation. C'est l'usage qui, en dernier lieu, aurait été assigné à une pierre gravée dans un village du Quercy. 


\section{Situation et description de la pierre de Teyssieu}

La pierre dont il va être question est située dans le bourg de Teyssieu (Lot, arrondissement de Figeac, canton de Bretenoux). Elle est intégrée dans le mur d'une propriété. Ce mur sert de soutènement à une rampe permettant l'accès au portail occidental de l'église du lieu, dédiée à sainte Marie-Madeleine. Cette pierre, de forme circulaire, taillée dans une roche granitique riche en quartz est de couleur blanchâtre (couleur L $92 \mathrm{du}$ Code des couleurs de sols de A. Cailleux). Elle mesure de 1,02 à 1,06 m de diamètre. On observe un léger chanfrein sur certaines parties de la périphérie. Peut-être s'agit-il d'une ancienne meule dormante.

Quoi qu'il en soit, sa face visible est ornée d'une figure gravée rectangulaire de 0,73 m de longueur et de 0,49 $\mathrm{m}$ de largeur, divisée en deux compartiments par deux lignes transversales gravées (fig. 1 et 2). Dans chacune des deux cases ainsi créées, perpendiculairement aux bordures longitudinales, s'étendent des sortes de barreaux d'une longueur de 0,13 à 0,14 m, s'achevant chacun par une cupule (fig. 1-2). Cette curieuse gravure, avec ces sortes d'échelles latérales a fait qualifier celles-ci de «scalariformes » dans une publication locale (Aquioupou 2000, fig. 2).

Ce type de gravure, s'il peut surprendre, est néanmoins connu. Il a déjà été relevé en Angleterre, au château d'Acre Castle, Norfolk, du XII ${ }^{\mathrm{e}}$ siècle, et identifié comme un tablier de jeu de trictrac (Coad \& Streeten 1982) (fig. 3).

\section{Un jeu bien attesté à l'époque médiévale}

L'objet de cette communication ne portant pas sur ce jeu, on nous pardonnera de ne pas entrer dans des détails trop étendus sur la manière dont il était pratiqué et sur l'évolution des tables ou tabliers sur lesquel (le)s opéraient les joueurs. Rappelons seulement que l'on s'accorde à voir dans le trictrac un avatar du jeu antique dit des douze lignes (duodecim scripta) (Alleu et Matignon 1964, p. 512-514; Bourgeois 2012, p. 33). Il est lui-même devenu l'ancêtre des jeux actuels de jacquet et de backgammon. Ces jeux, à l'instar du trictrac, comportent deux compartiments contenant chacun deux séries de six flèches (triangles ou simples lignes) sur lesquelles se déplacent des pions progressant selon le nombre de points obtenus par des lancers de dés à l'aide d'un cornet.

Plusieurs tabliers portatifs de trictrac, fabriqués en os, ont été retrouvés lors de fouilles récentes de sites d'habitats aristocratiques ou de centres de pouvoir médiévaux. Deux exemplaires datés des $\mathrm{X}^{\mathrm{e}}$-XII ${ }^{\mathrm{e}}$ siècles, découverts lors de la fouille du château de Mayenne (Mayenne) (Goret 2012, p. 57-62 et p. 71; Grandet 2012b), entre 1996 et 2000, avec d'autres pièces de jeu (Goret et Grandet 2012, p. 14-21), ont suscité, en 2012, une exposition sur le thème «Échecs et trictrac, les jeux de table au Moyen Âge » (Grandet et Goret 2012), dans le nouveau musée ouvert dans ce château en 2008.

On peut encore mentionner des éléments de deux autres tabliers de trictrac, du XII siècle, exhumés dans les fouilles urbaines de Saint-Denis (Seine-Saint-Denis) (Meyer et Wyss 1991, p. 103-113), neuf flèches d'un tablier de trictrac, des $X^{\mathrm{e}}-\mathrm{XII}$ e siècles, exhumées au cours de la fouille de la motte castrale de Boves (Somme), (Chandeveau 2002, p. 25-71; Racinet 2008), une flèche de tablier, du XIII ${ }^{\mathrm{e}}$ siècle, mise au jour lors de la fouille du donjon et de la haute-cour du château de Fréteval (Loir-et-Cher, (Leymarios 2001), trois fragments de tablier (deux éléments de bordure et un de flèche), des XI ${ }^{\mathrm{e}}-\mathrm{XII}^{\mathrm{e}}$ siècles, sortis de terre lors des fouilles au vieux château de Château-Thierry (Aisne), (Goret 1995, p. 143, 145, 146 ; fig. 75, 77), quatre flèches, $\mathrm{du} \mathrm{XI} \mathrm{e}^{\mathrm{e}}$ siècle, découvertes au cours de la fouille du château de Schlössel, à Klingenmünster (Allemagne, Rhénanie-Palatinat), (Barz 2008, p. 189-196) et un tablier, du $\mathrm{XI}^{\mathrm{e}}$ siècle, à Gloucester (Angleterre), (Stewart et Watkints 1984, p. 185-190). 
À tous ces vestiges de tabliers de jeu de trictrac, il convient d'ajouter les nombreux pions en corne de cervidés, en os ou en pierre, circulaires, polylobés ou ajourés, utilisés dans la pratique de ce jeu et dans celle du jeu de mérelle. On en connaît provenant du château de Mayenne (Mayenne) (Grandet et Goret 2012, p. 57-78), du castrum d'Andonne, à Villejoubert (Charente) (Bardiès-Fronty 2012, p. 66; Grandet 2012b, p. 106), de la cour du château de Blois (Loir-et-Cher) (Aubourg et Josset 2003 a, p. 169-216 ; 2003 b, p. 22-30), de la motte castrale de Boves (Somme) (Chandeveau 2002, p. 25-71; Racinet 2008), d'une fosse de la rue Sainte-Thérèse à Chartres (Eure-et-Loir) (Poplin 1977, p. 77-94; Jolly, Tuffier et Styczynski 2000), du donjon de Gouzon, à Chauvigny (Vienne) (Bourgeois 2003, p. 373-400 ; Eneau 1989, p. 39-49), de Curzon (Vendée) (Gendron 1979, p. 49-60), de Douai (Nord) (Demolon 1999 et 2010), de Fréteval (Loir-et-Cher) (Leymarios 2001), de Loisy (Saône-et-Loire) (Barthélémy et Hurou 1987, p. 168-173 ; Boidard 2002 ; Goret et Poplin 2011, p. 109-121; Hurou 1973, p. 27-37 ; Hurou 1974, p. 409-410), de la fouille de la place Baudoyer à Paris (Grandet et Goret 2012, p. 134-135), du site de La Mothe à Pineuilh (Gironde) (Grandet et Goret 2012, p. 136 et 139), de Saint-Denis (Seine-Saint-Denis) (Grandet et Goret 2012, p. 142-144 ; Meyer et Wyss 1991, p. 103-113) et du château de Tours (Indre-et-Loire) (Grandet et Goret 2012, p. 150-152).

À cette énumération, il convient d'ajouter bon nombre d'autres pions médiévaux conservés dans les musées français. Plusieurs d'entre eux, conservés à Paris (Musée de Cluny-Musée national du Moyen Âge, Bibliothèque nationale de France, Département d'histoire de l'art et d'archéologie de la Ville de Paris, Musée du Louvre) et à Niort (Musée du Donjon) ont été présentés en 2012 et 2013 lors de l'exposition «L'art du jeu, jeu dans l'art ", au Musée de Cluny, à Paris (Bardiès-Fronty et Dunn-Vaturi 2012, p. 70, 71 et 139). Passé le Moyen Âge classique, la pratique du jeu de trictrac semble s'être maintenue puisque l'on conserve au Musée Rolin, à Autun (Saône-et-Loire), un fort beau tablier en marbre blanc incrusté de flèches de pierre alternativement noires et rouges. Cet objet, daté des environs de 1500, est attribué à un atelier d'Italie du Nord (Schädler 2012, p. 149). Aux temps modernes, $\mathrm{XVII}^{\mathrm{e}}$-XIII ${ }^{\mathrm{e}}$ siècles, gravures et peintures mettent fréquemment en scène des joueurs de trictrac ou de jacquet.

\section{Utilisation du tablier de trictrac gravé sur pierre de Teyssieu}

La plupart des tabliers de trictrac médiévaux mesurent, dans leurs dimensions restituées, de 50 à $60 \mathrm{~cm}$ de longueur et de 35 à $40 \mathrm{~cm}$ de largeur, ce qui en fait des jeux aisément transportables, pouvant être déposés sur une table. Il n'en va, évidemment, pas de même de la dalle circulaire de Teyssieu. Sa matière, le granite, et ses dimensions, de 1,02 à 1,06 $\mathrm{m}$ de diamètre, en font un support de jeu d'extérieur, même si le trictrac est habituellement qualifié de jeu de table. On pourrait imaginer qu'elle ait été disposée sur la place principale du bourg où les passants désœuvrés auraient pu l'utiliser à leur convenance. Si cette hypothèse n'est pas à exclure, il convient néanmoins d'observer que la plupart des jeux de trictrac médiévaux attestés en France le sont, on l'a vu précédemment, sur des sites aristocratiques ou des lieux de pouvoir, le plus souvent des châteaux. Si la plupart des tabliers de jeu connus sont en matériaux nobles ou précieux, on ne peut exclure que, même sur des sites castraux, il ait pu en exister des exemplaires réalisés en des matériaux moins coûteux, en bois par exemple, ou gravés dans la pierre pour occuper la garnison.

Or, à Teyssieu, existait un château fort tenu par la famille seigneuriale des Bonafos, vassaux des vicomtes de Turenne. De ce château fort subsiste un donjon de plan quadrangulaire, datant $\mathrm{du} \mathrm{XIII}$ e siècle, communément nommé «la tour», qui vient d'ailleurs de bénéficier d'une utile et judicieuse restauration (d'Alauzier 1958, p. 38-43; Pressouyre 1989, p. 71-74). On peut donc imaginer que la dalle circulaire gravée du tablier de trictrac ait été installée dans la cour du château. Cette éventualité induit un constat: cette pierre n'aurait alors été déplacée que d'une faible distance. La propriété, 
dans le mur de laquelle elle est intégrée, n'est qu'à quelques dizaines de mètres du site sur lequel s'élevait l'ancien château.

Quoi qu'il en soit, dans l'état actuel, la date d'utilisation de ce tablier de trictrac est difficile à déterminer. Toutefois, on a vu plus haut qu'un autre tablier gravé sur pierre est connu dans un château anglais du XII ${ }^{\mathrm{e}}$ siècle. L'analogie de matière et de situation autorisent peut-être à envisager l'hypothèse d'une origine médiévale pour le tablier de trictrac de Teyssieu. D'autant qu'à l'occasion de la restauration récente de la tour de Teyssieu, une peinture murale représentant un tournoi dans lequel s'affrontent deux chevaliers, a été mise au jour. Cette découverte établit de manière forte que l'esprit d'activités élitistes, auquel semble se rattacher le jeu de trictrac, avait bien cours dans ce château fort au Moyen Âge.

\section{Le remploi de la dalle gravée de Teyssieu}

À une époque difficile à situer, peut-être le XVIII ${ }^{\mathrm{e}}$ ou le XIX $\mathrm{X}^{\mathrm{e}}$ siècle, mais qui a laissé des souvenirs qui se sont transmis jusqu'à l'époque contemporaine, la dalle de Teyssieu a connu un remploi particulièrement original. Une érudite locale, se fondant sur le témoignage qu'elle a recueilli auprès d'une ancienne habitante aujourd'hui décédée, explique comment le tablier de trictrac de Teyssieu, avec ses petites cupules à l'extrémité des flèches, a été remployé :

«Lorsqu'un berger amenait paître son troupeau dans les pâturages (probablement des communs), il devait déposer un petit caillou dans [une] cupulette, ce faisant le pâtre indiquait la position du pacage occupé par ses bêtes. » (Aquioupou 2000, p. 100-101 et fig. 2, pl. XXVII)

On peut se demander quel était l'intérêt de savoir où se trouvait tel ou tel troupeau. Sur ce point encore l'érudite consœur déjà citée apporte une réponse :

"Ce système de repérage, à la fois simple et astucieux, avait un double but : permettre une bonne répartition du bétail dans les pâturages et empêcher le mélange des troupeaux. » (ibid., p. 101)

Ce mélange des bêtes de différents propriétaires était un des problèmes posés par l'usage collectif de certains pâturages ou de certains points d'eau où les animaux pouvaient s'abreuver. C'est peut-être pourquoi, à Teyssieu, près des lieux-dits Las Places (espace public) et Touron (la source) existait un champ dit Triadou, permettant, si besoin était, de trier les troupeaux (Pressouyre 1989, p. 317).

On perçoit donc dans cet exemple de communication sans écriture, ni mots, ni sons, l'ingéniosité d'une population paysanne qui a su procéder à l'utile remploi d'une pierre probablement vouée auparavant (car si la provenance du château est présumée, elle n'est pas prouvée) à un loisir vraisemblablement étranger à la culture rurale autochtone. Ce support, réapproprié pour la communication, présentait en outre plusieurs avantages. D'abord, il était codé, sa connaissance pouvait n'être éventuellement réservée qu'aux seuls propriétaires et pâtres des troupeaux ou à la seule population de la localité, excluant ainsi les étrangers. Par ailleurs, cette communication n'utilisant pas l'écriture présentait un énorme avantage en des époques où la lecture et l'écriture n'étaient pas encore généralisées.

Ce tablier de trictrac remployé se présentait donc comme un support de communication à double vocation. Sa première attribution n'était ni plus ni moins que de faire office de carte géographique, puisque chaque flèche $\mathrm{du}$ jeu originel équivalait à la désignation d'un lieu précis et particulier. Sa seconde utilisation, découlant de la première, était une géolocalisation, comme l'on dit de nos jours, des troupeaux. Peut-être peut-on envisager, 
en poussant le concept à son maximum, que le caillou identifiant chaque troupeau ou chaque pâtre ait été différencié des autres. Le procédé avait toutefois ses limites puisqu'il ne pouvait désigner que vingt-quatre sites de pacage. Il convenait aussi qu'il fût géré avec rigueur et tenu à jour quotidiennement. Mais, faisons confiance au bon sens paysan, il n'est pas douteux qu'il l'ait été. Admirons aussi ce même bon sens qui, d'une pierre devenue sans objet, sut faire un ingénieux mode de transmissions de données.

\section{Bibliographie}

Alauzier L. d' (1958). La tour de Teyssieu, Bulletin de la Société des Études littéraires, scientifiques et artistiques $d u$ Lot, LXXIX, p. 38-43.

Alleu R. et Martignon R. (1964). Dictionnaire des jeux, Tchou, Paris, notice "Trictrac », p. 512-514.

Aquioupou Y. (2000). Un passé à découvrir en Ségala. Le canton de Sousceyrac et ses abords, Sousceyrac d'hier à aujourd'hui, Sousceyrac, p. 101.

Aubourg V. et Josset D. (2003 a). Le site du promontoire du château de Blois du VIII au $\mathrm{XI}^{\mathrm{e}}$ siècle. Seconde partie : le mobilier non céramique, Revue archéologique du Centre de la France, t. 42, p. 169-216.

Aubourg V. et Josset D. (2003 b). Blois en l'an mil, Archéologia, n 368, p. 22-30.

BARDiÈs-Fronty I. (2012). Pions, in I. BARDiès-Fronty et A.-E. DunN-VATURI (dir.) Art $d u$ jeu, jeu dans l'art, de Babylone à l'Occident médiéval, Catalogue de l'exposition du musée de Cluny, RMN, Paris, notices 28-29, p. 66.

BARDiÈs-Fronty I. et DunN-VAturi A.-E. (dir.) (2012). Jeu dans l'art, l'art du jeu, de Babylone à l'Occident médiéval, Catalogue de l'exposition du musée de Cluny, Paris 28 novembre 2012-4 mars 2013, RMN, Paris, 160 p.

BARTHÉLÉMY A. et Hurou G. (1987). La motte de Loisy, Bourgogne médiévale : la mémoire du sol, catalogue de l'exposition, Dijon, p. 168-173.

BARZ D. (2008). Schlössel bei Klingenmünster. Befunde und Funde einer salierzeitlichen Burg", Archäologie mittelalterlicher Burger, Mittelungen der Deutscher Gesellschaft für Archäologie des Mittelalters und der Neuzeit, t. 20, p. 189-196.

BOIDARD V. (2002). Loisy, une motte castrale de l'an mil (fouilles de G. Berthoud et G. Hurou), Mâcon, Bourgogne archéologique, $\mathrm{n}^{\circ} 19$.

BourgeOIS L. (2003). Pièce de jeu et milieu aristocratique dans le Centre-Ouest de la France ( $\mathrm{X}^{\mathrm{e}}-\mathrm{XII}{ }^{\mathrm{e}}$ siècles), Aquitania, t. 18, p. 373-400.

Bourgeois L. (2012). À propos des jeux de trictrac et de mérelles, in M. GRANDET et J.-F. GORET (dir.) Échecs et trictrac, fabrication et usage des jeux de table au Moyen Âge, Catalogue de l'exposition du musée de Mayenne, Errance et Actes Sud, Paris et Arles, p. 33.

CAilleux A. (s.d.). Code des couleurs de sols, Paris, Boubée.

Chandeveau F. (2002). La motte castrale de Boves (Somme). Tabletterie et petits artefacts ( $\mathrm{x}^{\mathrm{e}}-\mathrm{XVI}^{\mathrm{e}}$ siècles), Revue archéologique de Picardie, 1-2, p. $25-71$.

COAD J.G. et SteETEN A.D.F. (1982). Excavations at Castle Acre Castle, Norfolk, 1972-77. Country house and castle of the Norman Earls of Surrey, Archaeological Journal, 118, p. 138-301. 
DEMOLON P. (1999). 20 ans d'archéologie dans le Douaisis, catalogue d'exposition, 10 au 19 sept. 1999, Archaeologia Duacensis, 16.

Demolon P. (2010). Archéologie en Douaisis. Regards sur un territoire: l'Ostrevent, Douai, Communauté d'agglomération du Douaisis, Archaeologia Duacensis, 30.

ENEAU T. (1989). Fouille au donjon de Gouzon (Chauvigny, Vienne), Le pays chauvinois, $\mathrm{n}^{\circ}$ 27, p. 44-45.

Gendron C. (1979). Jetons et jeux de table romans au musée du Pilori de Niort et dans l'Ouest, Bulletin de la Société historique et scientifique des Deux-Sèvres, $2^{\mathrm{e}}$ série, t. 12, p. 49-60.

GORET J.-F. (1995). Le mobilier osseux travaillé découvert sur le site du "Vieux Château » de Château-Thierry. IX ${ }^{e}$-XII ${ }^{e}$ siècle, Mémoire de maîtrise, Université de Paris I, sous la direction de L. Pressouyre, t. 2, p. 141-149.

GORET J.-F. et POPLIN F. (2011). Le mobilier de qualité en matières dures d'origine animale $\mathrm{du} \mathrm{XI} \mathrm{I}^{\mathrm{e}}$ siècle découvert sur le site de la motte de Loisy (Saône-et-Loire), Bulletin de la Société nationale des Antiquaires de France, séance du 2 juin 2004, p. 109-121.

GORET J.-F. (2012). Les pièces de jeu du château de Mayenne, in M. GRANDET et J.-F. GORET (dir.) Échecs et trictrac, fabrication et usage des jeux de table au Moyen Âge, Catalogue de l'exposition du musée de Mayenne, Errance et Actes Sud, Paris et Arles, p. 57-62 et 71.

GORET J.F. et GRANDET M. (2012). Échecs et trictrac, les jeux de table au Moyen Âge, Archéologia, 501, p. 14-21.

GRANDET M. et GORET J.-F. (dir.) (2012). Échecs et trictrac, fabrication et usage des jeux de table au Moyen Âge, Catalogue de l'exposition du musée de Mayenne, Errance et Actes Sud, Paris et Arles, $160 \mathrm{p}$.

GRANDET M. (2012a). Un écrin unique pour une collection exceptionnelle, in M. Grandet et J.-F. GORET (dir.) Échecs et trictrac, fabrication et usage des jeux de table au Moyen Âge, Catalogue de l'exposition du musée de Mayenne, Errance et Actes Sud, Paris et Arles, p. 19-22.

GRANDEt M. (2012b). Pièces d'échecs et jeu de trictrac ou de mérelles, dés, mérelliers, in I. BARDiÈs-FrontY et A.-E. DunN-VATURI (dir.) Art du jeu, jeu dans l'art, de Babylone à l'Occident médiéval, Catalogue de l'exposition du musée de Cluny, RMN, Paris, notices 9497, p. 105.

HuROU G. (1973). Le point sur cinq années de fouille à Loisy, Bulletin du Groupement archéologique du Mâconnais, $\mathrm{n}^{\circ}$ 6, p. 27-37.

HuROu G. (1974). Saône-et-Loire, Loisy, Archéologie médiévale, t. III-IV, p. 409-410.

JOLly D., TUFFIER A. et STYCZYNSKI C. (2000). Premières lumières. Chartres de ses origines au Moyen Âge, Chartres, Ville de Chartres.

LEYMARIOS C. (2001), Fréteval. Histoire d'une forteresse médiévale, Cherche-Lune, Vendôme, pl. XIV.

MEYER N. et WYSS M. (1991). Un jeu de table du XII ${ }^{\mathrm{e}}$ siècle provenant de Saint-Denis, Archéologie médiévale, XXI, p. 103-113. 
POPLIN F. (1977). Analyse de matières de quelques ivoires d'art, Méthodologie appliquée à l'industrie de l'os préhistorique, actes du colloque international du CNRS, Paris, Éditions du CNRS, p. 77-94.

Pressourre F. (1989). Teyssieu des origines à 1800, Association des Amis du Passé de SaintCéré, Saint-Céré, 408 p.

RACINET P. (2008). Le site castral de Boves du $X^{\mathrm{e}}$ au XVII ${ }^{\mathrm{e}}$ siècle. Bilan des recherches archéologiques 2001-2006, Revue archéologique de Picardie, 1-2, 168 p.

SCHÄLDER U. (2012). Un plateau de trictrac, in I. BARDIÈS-FrONTY et A.-E. DUNN-VATURI (dir.) Art du jeu, jeu dans l'art, de Babylone à l'Occident médiéval, Catalogue de l'exposition du musée de Cluny, RMN, notice 163, p. 149.

STEWART I.J. et WATKINS M.J. (1984). An $11^{\text {th }}$ Century bone tabula set from Gloucester, Medieval Archaeology, XXVIII, p. 185-190.

\section{Illustrations}

Figure 1: Dans le mur où elle est intégrée, la dalle circulaire gravée de Teyssieu (diamètre : $1 \mathrm{~m}$. environ, cliché G.R. Delahaye).

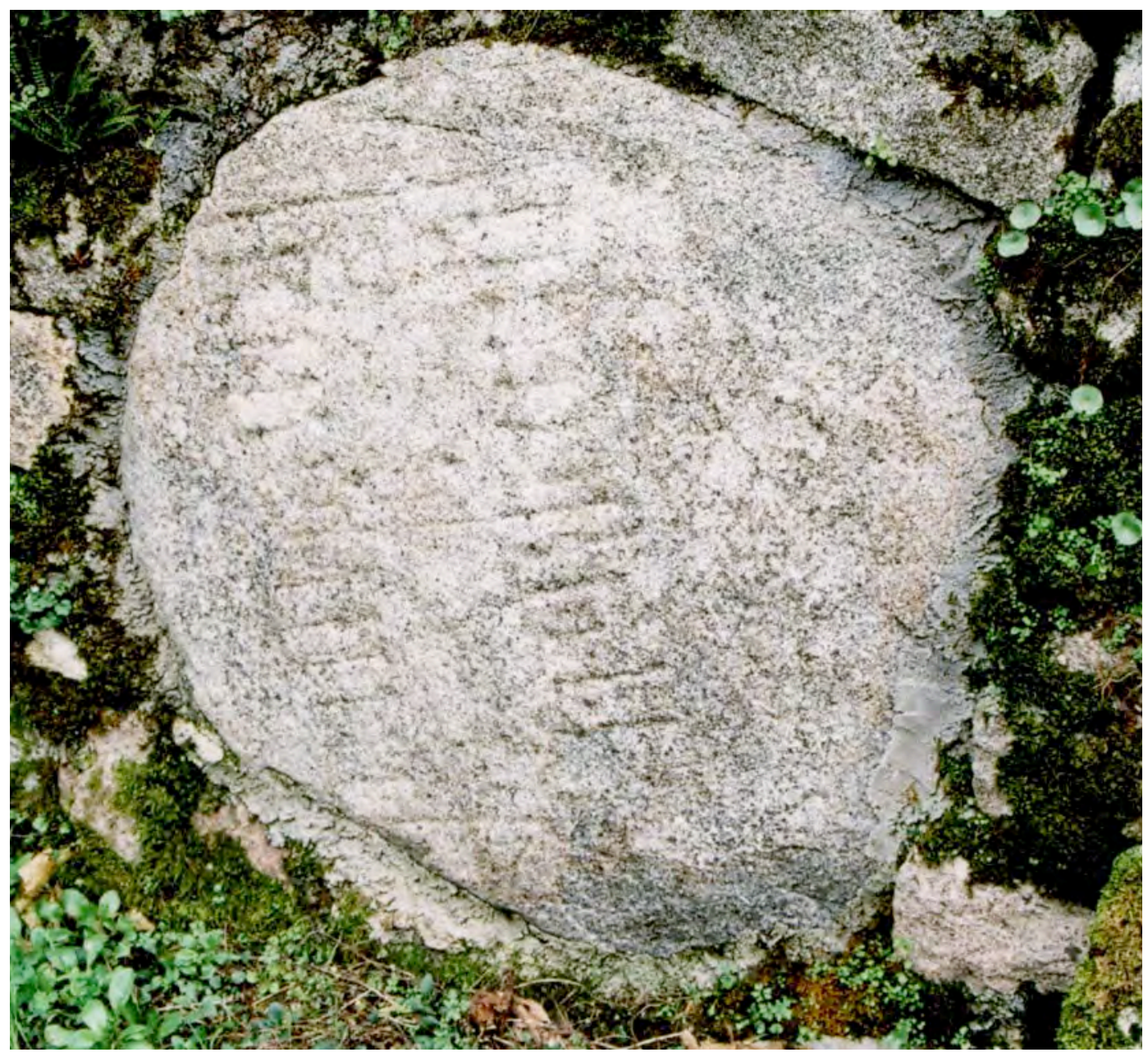


Figure 2 : La dalle circulaire gravée de Teyssieu (Lot) (dessin G.R. Delahaye).

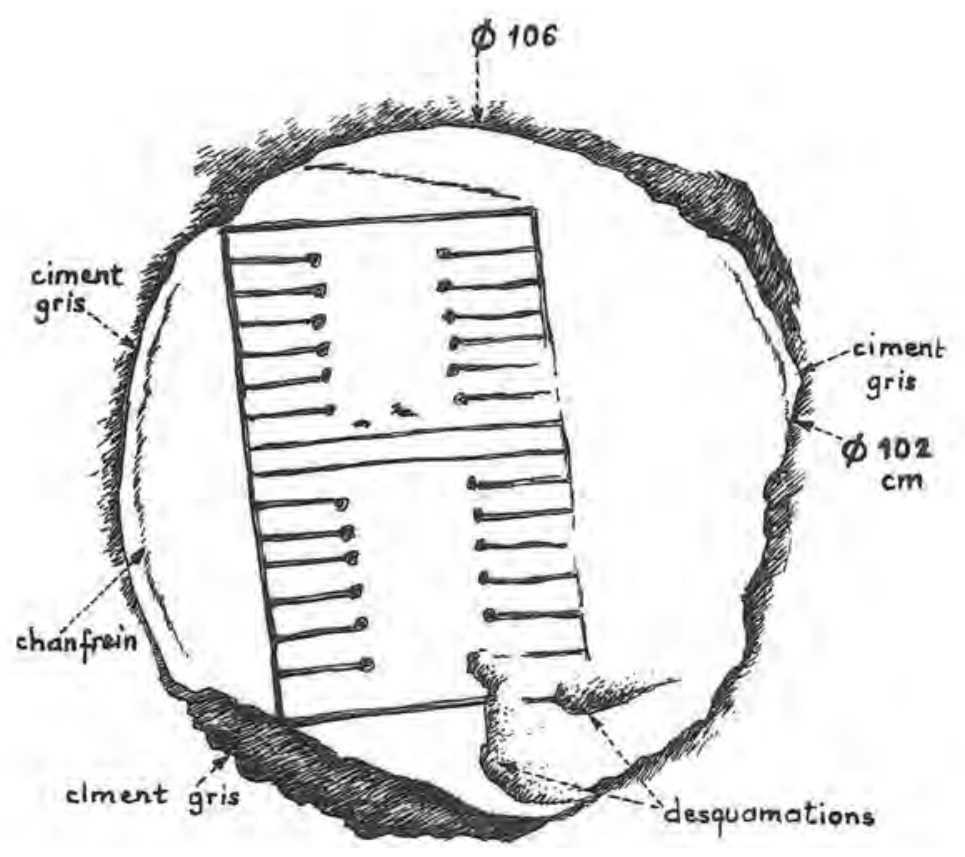

Figure 3: Le tablier de jeu de trictrac d'Acre Castle (Angleterre, Norfolk) (dessin G.R. Delahaye d'après le relevé publié par J.G. Coad et A.D.F. Steeten).

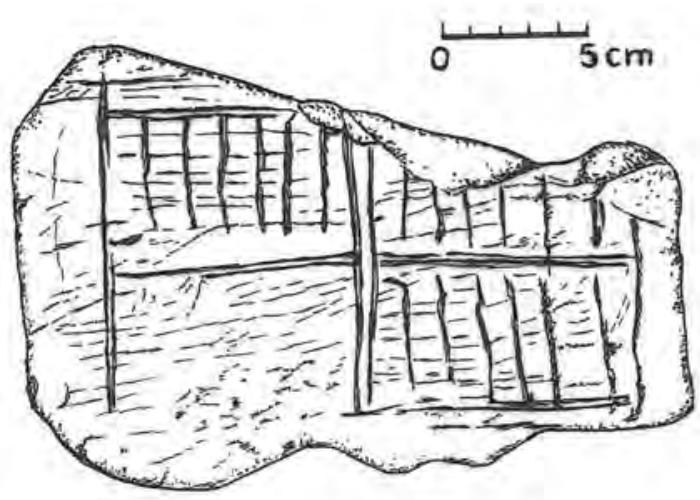

